\title{
One hundred and fifty years of ornithology in Sicily, with an unknown manuscript by Joseph Whitaker
}

\author{
Bruno Massa', Renzo lentile ${ }^{2 *},{\text { Arianna } \text { Aradis }^{3} \& \text { Salvatore Surdo }}^{4}$ \\ ${ }^{1}$ Department of Agriculture, Food ans Forest Sciences, University of Palermo, V.le Scienze 13, 90128 Palermo (retired); \\ e-mail: bruno.massa@unipa.it \\ ${ }^{2}$ Centro Universitario per la Tutela e la Gestione degli Ambienti Naturali e degli Agro-ecosistemi, University of Catania, \\ Via Santa Sofia 98, 95123 Catania (Italy); e-mail: renzoientile@unict.it \\ ${ }^{3}$ Istituto Superiore per la Protezione e la Ricerca Ambientale, Roma, Italy; e-mail: arianna.aradis@isprambiente.it \\ ${ }^{4}$ Department of Agriculture, Food ans Forest Sciences, University of Palermo, V.le Scienze 13, 90128 Palermo; \\ e-mail: salvatore.surdo@unipa.it \\ ${ }^{*}$ Corresponding author
}

\begin{abstract}
A new complete check-list of Birds of Sicily is presented in this paper, with a comparison with previous lists for a period of one hundred and fifty years. Further, an unknown manuscript by Joseph Whitaker "Birds of Sicily", dated back to ca. 1920, has been transcribed and is here presented integrally. Thus, lists of birds here presented have been separated as follows: i) Doderlein (1869-1874); ii) Whitaker (1920); iii) Iapichino \& Massa (1989), Lo Valvo et al. (1993) iv) Corso (2005), Ientile \& Massa (2008); v) 2010-2020: Massa et al. (2015) and personal observations; vi) long-term trend: personal observations. Overall, 437 species are listed. Out of 283 species regularly present in Sicily, over the long period here considered of one hundred and fifty years, 75 (26.5) resulted to maintain stable populations, 35 (12.4\%) resulted increasing, $12(4.2 \%)$ very increasing, $75(26.5 \%)$ with declining populations, 34 (12\%) with very declining populations and $11(3.9 \%)$ became extinct. Further, 41 species (14.5\%) showed a population trend different from the previous ones, that we classified as fluctuating. We discuss about seventy representatives of the previous categories.
\end{abstract}

KEY WORDS Birds of Sicily; chrono-checklists; trend; increasing; fluctuating; history.

Received 14.11.2020; accepted 21.12.2020; published online 15.02.2021

This paper is dedicated to the memory of the Sicilian ornithologist Angelo Priolo (1923-2006)

\section{INTRODUCTION}

Ornithology in Sicily has traditionally been much explored, numerous ornithologists have lived in Sicily and several ornithological publications starting since 1713 are available (e.g., Cupani, 1713); in the early 1800s Baldassare Palazzotto made a manu- script of Sicilian Ornithology, unfortunately so far never transcribed by anyone. In 1840 Benoit published the first almost complete list of birds, but undoubtedly the first complete and exhaustive work on the birds of Sicily is due to Doderlein (1869-1874), who in 1862 came to hold the chair of Zoology and Compared Anatomy at the University of Palermo, coming from the University of Modena, immediately after the Unification of Italy. In the last years of Doderlein's life, Joseph Whitaker (1850-1936), known as 'Pip', entrepreneur and winemaker, arrived from 
England to Sicily, who, thanks to his many activities, maintained a very high standard of living and devoted himself much to the ornithology, especially that of North Africa, but he collected also a lot of data on Sicilian birds.

More than thirty years ago the first author by chance found the list of mounted specimens of the collection of Joseph Whitaker, that was used for the catalogue of bird specimens preserved in that collection (Lo Valvo \& Massa, 2000). Together with that list also an interesting manuscript by Joseph Whitaker titled 'Birds of Sicily' was found (shortly cited by Iapichino \& Massa, 1989). During his life J. Whitaker collected an impressive number of birds (9549), which were preserved in a building within his "Villa Malfitano" at Palermo. In 1939, with permission of his daughter, Delia Whitaker, the English ornithologist David Bannerman took the types of species and subspecies described by Whitaker to the British Museum (Natural History). Twenty years after his death, in 1956, Delia presented all the skins (8136) to the National Museum of Scotland, Edinburgh; and then, in 1968, all the mounted specimens (1413, mainly from Italy) to the Ulster Museum, Belfast (see also Massa, 1987a, 1995; R. McGowan in Lo Valvo \& Massa, 2000). The Whitaker collection consisted mostly of specimens from Europe (particularly Italy) and North Africa, where he collected many birds; additionally, at the end of the $19^{\text {th }}$ Century, Whitaker bought the skin collection of Lord Lilford, including 3277 specimens from Europe and Mediterranean countries.

The manuscript by Whitaker consists of eighteen pages and, following the dates recorded in the text, it very probably was compiled between 1910 and 1920; Whitaker (1905) published his very important two volumes on the Birds of Tunisia, where he reported also information on some Sicilian birds, but never he published a check-list on the birds of the island, even if he published many notes on this subject (Whitaker, 1882, 1896, 1899a, 1899b, 1899c, 1899d, 1901, 1902, 1903, 1904a, 1904b, 1904c, 1910a, 1910b, 1910c).

When Whitaker was still alive, other people worked with care on the ornithology in Sicily, namely Antonino Trischitta (1892-1966), Carlo Orlando (1898-1976) and Angelo Priolo (1923-2006), who wrote many papers on Sicilian birds (for a complete Sicilian ornithological bibliography see http://www.ornitologiasiciliana.it/la-bibliografia-ornitologica-siciliana.html).
Currently, many ornithologists and birdwatchers live or visit regularly Sicily and the ornithological knowledge has grown very much, mainly with the observation of rare or accidental birds which pass over the island or the islets surrounding it. Breeding birds are well known, and since 1980s three different atlases of Sicilian breeding birds have been carried out (Massa, 1985; Lo Valvo et al., 1993; Ientile $\&$ Massa, 2008), the second of them treating also wintering birds. Further, two annotated Sicilian check-lists were published in the last thirthy years (Iapichino \& Massa, 1989; Corso, 2005). Thus, today the ornithology in Sicily is well known and it is the time to try a synthesis of the population trends through the long period of time between the half of 1800 and nowadays.

With the aims of presenting the present status of Sicilian birds, as well as their trend over one hundred and fifty years, we decided first to transcribe the list of Whitaker, written with a very difficult handwriting, then to compare the bird status recorded by Whitaker with the previous author, Doderlein (1869-1874), and with more recent authors who published check-lists at the end of the past century (Iapichino \& Massa, 1989) and on the early years of the present century (Corso 2005; Ientile \& Massa, 2008). Finally, we carried out our present check-list of Sicilian birds, with annotations on the status. This allowed us to have a trend over the long period examined for all the birds presently known from the island.

\section{MATERIAL AND METHODS}

\section{The list of former and present Sicilian birds}

The manuscript by Whitaker here transcribed follows the nomenclature of Dresser (1881), with only few exceptions; it was updated to the most recent list of Italian birds (Baccetti, Fracasso \& COI, 2020). The current scientific name is reported in bold, the synonym used by Whitaker and the transcribed handwritten text follow it. All the information reported by Whitaker were transcribed, when abbreviated, they were fully written (e.g., coll. = collection, $\mathrm{ob}^{\text {td }}=$ obtained); wrong words remained as they were written (e.g., Cinclus acquaticus instead of Cinclus aquaticus, Bottaurus instead of Botaurus, etc.). When the author for some species 
wrote 'do do' it means 'ditto', that is the text is the same of the previous species; thus, we copied it. For completeness, we present the original handwriting pages of Whitaker. For some species Whitaker did a reference to Doderlein (also Dod.), which is the paper published by this author between 1869 and 1874; he reported also 'Palermo museum', which is the museum established by Pietro Doderlein at the Palermo's University (see Massa et al., 2018).

The paper by Doderlein (1869-1874) used to write the list of the species cited by this author is the personal copy of Pietro Doderlein, containing many handwritten annotations, that were useful to complete the present paper. For example, Doderlein did not list the Gannet Morus bassanus, but he wrote it in ink in the page of Cormorants, having obtained a specimen in 1882; however, in 1883 published the rare record (Doderlein, 1883). This is an interesting clue of the former rarity in the Mediterranean of a species regularly present today.

Lists of birds are separated as follows: i) Doderlein (1869-1874); ii) Whitaker (1920); iii) Iapichino \& Massa (1989), Lo Valvo et al. (1993); iv) Corso (2005), Ientile \& Massa (2008); v) 2010-2020: Massa et al. (2015), pers. obs.; vi) long-term trend: pers. obs.

In the present check-list only the subspecies confirmed for Sicily are reported; very likely other subspecies are passage migrants through the island and will be detected in the next years. Only accepted species by the Italian Committee of Homologation (C.O.I.) are here reported, only except for Balearica pavonina, explained in the Discussion.

This check-list will be presented and regularly updated in the web site www.ornitologiasiciliana.it.

\section{The trend of Sicilian birds over one hundred and fifty years}

After to have carrying out the check-list trend since the half of 1800 (Doderlein, 1869-1874) to nowadays, we established seven categories of the population trend: i) stable (st), when no differences have been noticed; ii) declining (dec), when objective diminution of the population was observed; iii) very declining (vdec), when a notable diminution of the population was observed; iv) increasing (in), when the population, originally scarce, became more abundant; v) very increasing (vin), when the population passed from rare or scarce to common or very common; vi) fluctuating (flu), when no pre- cise trend was observed, in particular neither declining nor increasing, but with numbers that fluctuated over the years; vii) extinct species (Ex), those presently vanished, but formerly resident breeder.

We reported the occurrence by every author as follows: very common (vc); common (c); scarce or fairly common (sc); rare (r); very rare (vr); irregular (irr), that is a species observed 3-8 times in ten years; occasional (occ), that is a species observed 1-2 times in ten years; reintroduced (reintr); alien species (al); the species observed every year or 9 times in ten years are considered regularly present in Sicily. In the final discussion, and also in the frequency of occurrence of each category irregular or accidental taxa were not considered and subspecies were not considered as separate taxa.

We would highlight that the trend presented in the present paper is referred to the wide time spam of one hundred and fifty years, and it should not be regarded as a more recent trend.

\section{RESULTS}

\section{The manuscript of Joseph Whitaker on the Birds of Sicily}

The transcription of the manuscript dated ca. 1920 is entirely reported in the Appendix 1, where also the original eighteen pages of the Whitaker list are presented.

\section{The list of breeding birds}

We report in the Table 1 the complete list of Sicilian birds. Overall, the number of bird species found over one hundred and fifty years amounts to 437, of which eleven became extinct (only one belongs to Passeriformes, the others are non Passeriformes). In the caption of the table abbreviations used in the different columns are explained; when it is reported M, W sc it means that the taxon is considered scarce both as migrant and wintering, conversely $\mathrm{M} \mathrm{sc}, \mathrm{Wr}$ means that it is considered scarce as migrant, rare as wintering.

Table 2 shows the list of the 29 alien species detected in Sicily; no proofs of breeding is known for them, except for Streptopelia roseogrisea (Corso, 2005). 
Table 1. Check-list of the Birds of Sicily from the half of 1800 to 2020.

Status: $\mathrm{A}=$ Accidental; $\mathrm{B}=$ Breeder; $\mathrm{Ex}=$ extinct; $\mathrm{M}=$ Migrant; $\mathrm{RB}=$ Resident Breeder; $\mathrm{SB}=$ Summer Breeder; $\mathrm{W}=\mathrm{Win}-$ tering; $\mathrm{vc}=$ very common; $\mathrm{c}=$ common; $\mathrm{sc}=$ scarce or fairly common; $\mathrm{r}=\mathrm{rare} ; \mathrm{vr}=$ very rare; irr $=$ irregular; occ $=\mathrm{occa}-$ sional; reintr = reintroduced; al = alien species. References. Species sequence and nomenclature: Baccetti, Fracasso \& COI, 2020; Column A: Doderlein, 1869-1874; Giglioli, 1907; column B: Whitaker, 1920, unpubl.; column C: Iapichino \& Massa, 1989; Lo Valvo et al., 1993; Lo Valvo \& Massa, 1999; column D: Corso, 2005; Ientile \& Massa, 2008; column E: 20102020, Massa et al., 2015, pers. obs.; column F (trend): pers. obs. Trend over 150 years. in = increasing; vin = very increasing; $\mathrm{dec}=$ declining; $\mathrm{vdec}=$ very declining; $\mathrm{st}=$ stable; flu = fluctuating .

\begin{tabular}{|c|c|c|c|c|c|c|c|}
\hline & Species & $\mathbf{A}$ & B & $\mathrm{C}$ & D & $\mathbf{E}$ & $\mathbf{F}$ \\
\hline 1 & Coturnix coturnix & M vc, B c & $\mathrm{M} \mathrm{vc}, \mathrm{RB} \mathrm{c}$ & $\mathrm{Mc}, \mathrm{RB} \mathrm{sc}$ & $\mathrm{SB}$ sc, $\mathrm{Mc}, \mathrm{Wr}$ & $\mathrm{SB}$ sc, $\mathrm{Mc}, \mathrm{Wr}$ & vdec \\
\hline 2 & Alectoris graeca whitakeri & $\mathrm{RB} v \mathrm{c}$ & $\mathrm{RB} \mathrm{c}$ & RB sc & $\mathrm{RB} \mathrm{sc}$ & RB sc & vdec \\
\hline 3 & Francolinus francolinus & Ex (ca. 1870) & Ex & Ex & Ex & Ex & Ex \\
\hline 4 & Phasianus colchicus & $\begin{array}{l}\text { Ex (introduced } \\
\text { in 1800) }\end{array}$ & - & $?$ & - & - & - \\
\hline 5 & Oxyura leucocephala & $\mathrm{M}, \mathrm{W}$ c, B occ & $\mathrm{M}, \mathrm{W} \mathrm{sc}$ & A & A & A & vdec \\
\hline 6 & Cygnus olor & $\mathrm{Mr}$ & - & $\mathrm{M}, \mathrm{W}$ irr & M, W irr & $\mathrm{M}, \mathrm{W}$ irr, $\mathrm{r}$ & - \\
\hline 7 & Cygnus cygnus & $\mathrm{M}, \mathrm{W} \mathrm{r}$ & M, W occ & $\mathrm{M}, \mathrm{W}$ irr & $\mathrm{A}$ & A & - \\
\hline 8 & Cygnus columbianus bewickii & $\mathrm{A}$ & - & A & A & A & - \\
\hline 9 & Branta bernicla & - & $\mathrm{M}, \mathrm{W} \mathrm{sc}$ & A & A & A & - \\
\hline 10 & Branta ruficollis & - & - & - & A & A & - \\
\hline $11 \mathrm{a}$ & Anser anser anser & $\mathrm{M}$ vr & $\mathrm{M}, \mathrm{W} \mathrm{r}$ & $\mathrm{M}, \mathrm{W}$ sc & $\mathrm{M}, \mathrm{W} \mathrm{sc}$ & $\mathrm{M}, \mathrm{W}$ irr, $\mathrm{r}$ & in \\
\hline $11 \mathrm{~b}$ & Anser anser rubrirostris & - & - & $\mathrm{A}$ & A & A & - \\
\hline 12 & Anser fabalis & $\mathrm{M}, \mathrm{W} \mathrm{c}$ & $\mathrm{M}, \mathrm{W} \mathrm{r}$ & A & $\mathrm{M}, \mathrm{W}$ occ & $\mathrm{M}, \mathrm{W}$ occ & dec \\
\hline 13 & Anser albifrons & - & $\mathrm{M}, \mathrm{W} \mathrm{r}$ & $\mathrm{M}, \mathrm{W}$ irr & M, W irr & $\mathrm{M}, \mathrm{W}$ irr, $\mathrm{r}$ & st \\
\hline 14 & Anser erythropus & - & - & A & A & A & - \\
\hline 15 & Somateria mollissima & - & - & - & A & A & - \\
\hline 16 & Melanitta fusca & $\mathrm{A}$ & $\mathrm{A}$ & - & A & A & - \\
\hline 17 & Melanitta nigra & - & - & $\mathrm{A}$ & A & A & - \\
\hline 18 & Bucephala clangula & $\mathrm{M}, \mathrm{r}$ & $\mathrm{M}, \mathrm{W}, \mathrm{r}$ & A & A & A & vdec \\
\hline 19 & Mergellus albellus & $\mathrm{M}, \mathrm{W} \mathrm{sc}$ & $\mathrm{M}, \mathrm{W}$ occ & A & A & A & vdec \\
\hline 20 & Mergus merganser & $\mathrm{M}, \mathrm{r}$ & - & $\mathrm{A}$ & $\mathrm{A}$ & $\mathrm{A}$ & - \\
\hline 21 & Mergus serrator & $\mathrm{M}, \mathrm{W} \mathrm{c}$ & $\mathrm{M}, \mathrm{W}$ occ & $\mathrm{M}, \mathrm{W}$ sc & $\mathrm{M}, \mathrm{W}$ sc & $\mathrm{M}, \mathrm{W} \mathrm{sc}$ & dec \\
\hline 22 & Tadorna tadorna & $\mathrm{M}, \mathrm{W} \mathrm{sc}$ & $\mathrm{M}, \mathrm{W} \mathrm{c}$ & $\mathrm{M}, \mathrm{W} \mathrm{sc}$ & $\mathrm{M}, \mathrm{W} \mathrm{sc}, \mathrm{B} \mathrm{r}$ & $\mathrm{M}, \mathrm{W} \mathrm{sc}, \mathrm{B} \mathrm{r}$ & in \\
\hline 23 & Tadorna ferruginea & M vr & A & $M$ irr & M, W irr & $\begin{array}{l}\text { M, W irr, B } \\
\text { occ? }\end{array}$ & in \\
\hline 24 & Marmaronetta angustirostris & A & M irr & A & $\mathrm{M}, \mathrm{B} \mathrm{r}$ & $\mathrm{M}, \mathrm{B} \mathrm{r}$ & in \\
\hline 25 & Netta rufina & $\mathrm{M}, \mathrm{W} \mathrm{c}, \mathrm{B} \mathrm{sc}$ & $\mathrm{M}, \mathrm{W} \mathrm{c}, \mathrm{B} \mathrm{sc}$ & M sc & $\mathrm{M} \mathrm{r}, \mathrm{B}$ occ & Mr, W irr, B Ex & dec \\
\hline 26 & Aythya ferina & $\mathrm{M}, \mathrm{W} \mathrm{c}$ & $\mathrm{M}, \mathrm{W}$ vc & $\mathrm{M}, \mathrm{W} \mathrm{c}, \mathrm{B} \mathrm{sc}$ & $\mathrm{M}, \mathrm{W} \mathrm{c}, \mathrm{B}$ irr & $\mathrm{M}, \mathrm{W} \mathrm{sc}, \mathrm{B} \mathrm{r}$ & flu \\
\hline 27 & Aythya nyroca & $\mathrm{M}, \mathrm{W}$ c & $\mathrm{M}, \mathrm{W}$ vc, B? & $\mathrm{M}, \mathrm{W}, \mathrm{B}$ sc & $\mathrm{M}, \mathrm{W}$ sc, RB r & $\mathrm{M}, \mathrm{W}$ sc, RB r & vdec \\
\hline 28 & Aythya fuligula & $\mathrm{M}, \mathrm{W}$ vc & $\mathrm{M}, \mathrm{W} \mathrm{sc}$ & $\mathrm{M}, \mathrm{W} \mathrm{sc}$ & $\mathrm{M}, \mathrm{W} \mathrm{sc}$ & $\mathrm{M}, \mathrm{W} \mathrm{r}$ & vdec \\
\hline 29 & Aythya marila & - & - & $\mathrm{A}$ & $\mathrm{A}$ & $\mathrm{M}, \mathrm{W}$ occ & - \\
\hline 30 & Spatula querquedula & M vc & M vc & M c, B occ & Mc, W irr, B occ & $\mathrm{M} \mathrm{c}, \mathrm{B}, \mathrm{W}$ occ & dec \\
\hline 31 & Spatula clypeata & $\mathrm{M}, \mathrm{W} \mathrm{c}$ & $\mathrm{M}, \mathrm{W}$ sc & $\mathrm{M}, \mathrm{W}$ sc & M, W sc, B occ & $\mathrm{M}, \mathrm{W}$ sc & dec \\
\hline 32 & Mareca strepera & $\mathrm{M}, \mathrm{W}$ sc & $\mathrm{M}, \mathrm{W}$ c & $\mathrm{M}, \mathrm{W} \mathrm{sc}$ & $\mathrm{M}, \mathrm{W}$ sc, B occ & $\mathrm{M}, \mathrm{W}$ sc, B occ & flu \\
\hline 33 & Mareca penelope & $\mathrm{M}, \mathrm{W}$ ve & $\mathrm{M}, \mathrm{W}$ vc & $\mathrm{M}, \mathrm{W}$ sc & $\mathrm{M}, \mathrm{W}$ c & $\mathrm{M}, \mathrm{W}$ c & dec \\
\hline 34 & Anas platyrhynchos & $\mathrm{M}, \mathrm{W} \mathrm{vc}, \mathrm{B} \mathrm{sc}$ & $\mathrm{M}, \mathrm{W} \mathrm{vc}, \mathrm{B} \mathrm{sc}$ & $\mathrm{M}, \mathrm{W}, \mathrm{B}$ sc, & $\mathrm{M}, \mathrm{W} \mathrm{c}, \mathrm{RB} \mathrm{sc}$ & $\mathrm{M}, \mathrm{W} \mathrm{c}, \mathrm{RB} \mathrm{sc}$ & st \\
\hline
\end{tabular}




\begin{tabular}{|c|c|c|c|c|c|c|c|}
\hline 35 & Anas acuta & $\mathrm{M}, \mathrm{W} \mathrm{c}$ & $\mathrm{M}, \mathrm{W} \mathrm{c}$ & $\mathrm{M}, \mathrm{W} \mathrm{sc}$ & $\mathrm{M}, \mathrm{W} \mathrm{sc}$ & $\mathrm{M}, \mathrm{W} \mathrm{sc}$ & dec \\
\hline 36 & Anas crecca & $\mathrm{M}, \mathrm{W}$ sc & $\mathrm{M}, \mathrm{W}$ ve & $\mathrm{M}, \mathrm{W}$ sc & $\mathrm{M}, \mathrm{W}$ c, B occ & $\mathrm{M}, \mathrm{W} \mathrm{c}$ & flu \\
\hline 37 & Tachybaptus ruficollis & $\mathrm{RB} \mathrm{vc}$ & $\mathrm{RB}, \mathrm{M} \mathrm{c}$ & $\mathrm{RB}, \mathrm{M} \mathrm{c}$ & M, W RB c & $\mathrm{M}, \mathrm{W}, \mathrm{RB} \mathrm{c}$ & dec \\
\hline 38 & Podiceps grisegena & $\mathrm{A}$ & - & $\mathrm{A}$ & $\mathrm{A}$ & A & - \\
\hline 39 & Podiceps cristatus & $\mathrm{M}, \mathrm{W}, \mathrm{B} \mathrm{c}$ & $\mathrm{RB}, \mathrm{M}, \mathrm{W} \mathrm{c}$ & $\mathrm{M}, \mathrm{W}, \mathrm{B} \mathrm{sc}$ & $\mathrm{M}, \mathrm{W}, \mathrm{B} \mathrm{sc}$ & $\mathrm{RB}, \mathrm{M}, \mathrm{W}$ sc & dec \\
\hline 40 & Podiceps auritus & $\mathrm{A}$ & $\mathrm{M}, \mathrm{W}$ occ & $\mathrm{A}$ & $\mathrm{A}$ & $\mathrm{A}$ & - \\
\hline 41 & Podiceps nigricollis & $\mathrm{M}, \mathrm{W}$ ve & $\mathrm{RB}, \mathrm{Mc}$ & $\mathrm{M}, \mathrm{W} \mathrm{c}, \mathrm{B} \mathrm{r}$ & MW c, B r & $\mathrm{M}, \mathrm{W}$ sc, B r & dec \\
\hline 42 & Phoenicopterus roseus & $\mathrm{Mr}$ & M occ & M sc & $\mathrm{M}, \mathrm{W} \mathrm{c}$ & $\mathrm{M}, \mathrm{W} \mathrm{c}, \mathrm{B} \mathrm{r}$ & vin \\
\hline 43 & Columba livia & $\mathrm{RB} v \mathrm{c}$ & $\mathrm{RB} v \mathrm{c}$ & $\mathrm{RB} \mathrm{c}$ & $\mathrm{RB} \mathrm{c}$ & $\mathrm{RB} \mathrm{sc}$ & vdec \\
\hline 44 & Columba oenas & $\mathrm{M}, \mathrm{W} \mathrm{c}$ & $\mathrm{Mc}$ & $\mathrm{M}, \mathrm{W}$ irr, B vr & $\mathrm{M}, \mathrm{W}$ irr & M irr & vdec \\
\hline 45 & Columba palumbus & $\mathrm{M}, \mathrm{W} \mathrm{c}, \mathrm{B} \mathrm{sc}$ & $\mathrm{Mc}$ & $\mathrm{M}, \mathrm{W}, \mathrm{B} \mathrm{c}$ & $\mathrm{RB}, \mathrm{M}, \mathrm{W} \mathrm{c}$ & $\mathrm{RB}, \mathrm{M}, \mathrm{W}$ vc & vin \\
\hline 46 & Streptopelia turtur & $\mathrm{M} \mathrm{vc,} \mathrm{SB} \mathrm{c}$ & $\mathrm{Mvc}, \mathrm{SB} c, \mathrm{~W}$ irr & M, SB c & SB sc, Mc, W irr & $\mathrm{SB}, \mathrm{M} \mathrm{sc}$ & vdec \\
\hline 47 & Streptopelia orientalis & - & - & - & - & A & - \\
\hline 48 & Streptopelia decaocto & - & - & $\mathrm{A}$ & $\mathrm{RB} \mathrm{c}$ & $\mathrm{RB} v \mathrm{c}$ & vin \\
\hline 49 & Spilopelia senegalensis & - & - & $\mathrm{A}$ & $\begin{array}{c}\text { RB c } \\
\text { (Pantelleria) }\end{array}$ & $\begin{array}{c}\text { RB c (Pantel- } \\
\text { leria) RB vr } \\
\text { (mainland) }\end{array}$ & in \\
\hline 50 & Pterocles senegallus & - & A & A & A & A & - \\
\hline 51 & Pterocles alchata & A & - & - & $\mathrm{A}$ & A & - \\
\hline 52 & $\begin{array}{l}\text { Caprimulgus ruficollis } \\
\text { desertorum }\end{array}$ & - & A & A & A & A & - \\
\hline $53 \mathrm{a}$ & $\begin{array}{l}\text { Caprimulgus europaeus } \\
\text { meridionalis }\end{array}$ & $\mathrm{M} \mathrm{vc}, \mathrm{SB} \mathrm{sc}$ & $\mathrm{M} \mathrm{vc}, \mathrm{B} \mathrm{r}$ & M, B sc & $\begin{array}{l}\mathrm{SB}, \underset{\mathrm{Occ}}{\mathrm{M} \mathrm{sc}, \mathrm{W}} \\
\mathrm{O}\end{array}$ & $\mathrm{SB}, \mathrm{M} \mathrm{sc}$ & dec \\
\hline $53 \mathrm{~b}$ & $\begin{array}{l}\text { Caprimulgus europaeus } \\
\text { europaeus }\end{array}$ & - & - & - & $\mathrm{M} \mathrm{sc}$ & $\mathrm{M} \mathrm{sc}$ & - \\
\hline 54 & $\begin{array}{l}\text { Caprimulgus aegyptius } \\
\text { saharae }\end{array}$ & - & A & A & A & A & - \\
\hline 55 & Tachymarptis melba & $\mathrm{M}, \mathrm{SB} \mathrm{c}$ & $\mathrm{M}, \mathrm{SB} \mathrm{c}$ & M, SB sc & $\begin{array}{c}\mathrm{SB}, \mathrm{M} \mathrm{sc}, \mathrm{W} \\
\text { occ }\end{array}$ & $\begin{array}{c}\text { SB, M sc, W } \\
\text { occ }\end{array}$ & dec \\
\hline 56 & Apus affinis & - & - & - & A & $\mathrm{A}$ & - \\
\hline 57 & Apus pallidus brehmorum & Not recorded & Not recorded & M, SB sc & $\mathrm{SB}, \mathrm{M} \mathrm{sc}, \mathrm{W}$ irr & $\mathrm{SB}, \mathrm{M} \mathrm{c}$ & vin \\
\hline 58 & Apus apus & M, SB vc & M, SB vc & M, SB vc & SBc, Mvc, W irr & $\mathrm{SB}, \mathrm{M} \mathrm{c}, \mathrm{W}$ occ & dec \\
\hline 59 & Clamator glandarius & A & A & $\mathrm{Mr}$ & M irr, B irr & $\mathrm{SB}, \mathrm{Mr}$ & in \\
\hline 60 & Coccyzus americanus & - & - & $\mathrm{A}$ & $\mathrm{A}$ & $\mathrm{A}$ & - \\
\hline 61 & Cuculus canorus & $\mathrm{SB} \mathrm{c}, \mathrm{M} \mathrm{c}$ & SB c, M vc & $\mathrm{SB}, \mathrm{M} \mathrm{sc}$ & SB, M sc, W irr & SB, M sc, W irr & dec \\
\hline 62 & Rallus aquaticus & $\mathrm{RB} \mathrm{c}$ & $\mathrm{RB}, \mathrm{M} \mathrm{c}$ & $\mathrm{RB} \mathrm{sc}$ & $\mathrm{RB}, \mathrm{M}, \mathrm{W}$ sc & $\mathrm{RB} \mathrm{sc}$ & dec \\
\hline 63 & Crex crex & $\mathrm{M}, \mathrm{W} \mathrm{c}$ & M, W sc, B? & M sc & $\mathrm{M}, \mathrm{W}$ irr & M irr, W occ & vdec \\
\hline 64 & Porzana porzana & M c, B r (?) & M, W sc, B? & M sc & M sc, W irr & $\mathrm{M} \mathrm{sc}, \mathrm{W}$ occ & flu \\
\hline 65 & Zapornia parva & M sc & $\mathrm{M}, \mathrm{W}$ sc & M sc, B occ & $\mathrm{M}$ irr, $\mathrm{W}$ occ & M irr, W occ & dec \\
\hline 66 & Zapornia pusilla & M sc & $\mathrm{M}, \mathrm{W} \mathrm{sc}$ & $\mathrm{Mr}$ & M sc, W irr & M irr & dec \\
\hline $67 \mathrm{a}$ & Porphyrio porphyrio porphyrio & $\mathrm{RB} \mathrm{c}$ & RB sc, M & Ex (ca. 1960) & RB reintr & RB reintr & $\begin{array}{l}\mathrm{RB} \\
\text { reintr }\end{array}$ \\
\hline $67 b$ & $\begin{array}{l}\text { Porphyrio porphyrio } \\
\text { madagascariensis }\end{array}$ & A & $\mathrm{A}$ & A & A & A & - \\
\hline 68 & Porphyrio alleni & - & $\mathrm{A}$ & $\mathrm{A}$ & $\mathrm{A}$ & $\mathrm{A}$ & - \\
\hline 69 & Gallinula chloropus & $\mathrm{RB} v \mathrm{c}$ & $\mathrm{RB}, \mathrm{M} \mathrm{c}$ & $\mathrm{RB}, \mathrm{Mc}$ & RB vc, M, W c & $\mathrm{RB}, \mathrm{vc}, \mathrm{M}, \mathrm{W} \mathrm{c}$ & st \\
\hline 70 & Fulica cristata & A & A & A & A & A & - \\
\hline 71 & Fulica atra & $\mathrm{M}, \mathrm{W}$ vc, B sc & $\mathrm{M}, \mathrm{W}$ vc & $\mathrm{M}, \mathrm{W} \mathrm{c}, \mathrm{B} \mathrm{sc}$ & RB sc, M, W c & RB sc, M, W c & vdec \\
\hline 72 & Anthropoides virgo & $\mathrm{B}$ ? & $\mathrm{A}$ & $\mathrm{A}$ & $\mathrm{A}$ & $\mathrm{A}$ & - \\
\hline
\end{tabular}




\begin{tabular}{|c|c|c|c|c|c|c|c|}
\hline 73 & Grus grus & $\mathrm{Mc}$ & $\mathrm{Mc}$ & $\mathrm{Mc}$ & $\mathrm{M} \mathrm{c}, \mathrm{W} \mathrm{sc}$ & $\mathrm{M} \mathrm{c}, \mathrm{W} \mathrm{sc}$ & st \\
\hline 74 & Balearica pavonina & A & - & - & - & A & - \\
\hline 75 & Tetrax tetrax & $\mathrm{RB} \mathrm{c}, \mathrm{Mr}$ & $\mathrm{RB} \mathrm{r}$ & Ex (ca. 1965) & Ex & Ex & Ex \\
\hline 76 & Otis tarda & A & A & A & A & $\mathrm{A}$ & - \\
\hline 77 & Chlamydotis undulata & A & - & A & $\mathrm{A}$ & A & - \\
\hline 78 & Chlamydotis macqueenii & - & - & A & $\mathrm{A}$ & A & - \\
\hline 79 & Gavia stellata & A & M, W occ & $\mathrm{A}$ & $\mathrm{A}$ & A & - \\
\hline 80 & Gavia arctica & $\mathrm{M}, \mathrm{W} \mathrm{vr}$ & $\mathrm{M}, \mathrm{W}$ occ & A & M irr? & A & st \\
\hline 81 & Gavia immer & - & - & - & - & $\mathrm{A}$ & - \\
\hline 82 & Hydrobates pelagicus melitensis & RB c & $\mathrm{RB}$ sc & $\mathrm{RB} \mathrm{sc}$ & $\mathrm{RB} \mathrm{sc}$ & $\mathrm{RB} \mathrm{sc}$ & dec \\
\hline 83 & Hydrobates leucorhous & - & - & A & A & $\mathrm{A}$ & - \\
\hline 84 & Diomedea dabbenena & - & - & A & A & A & - \\
\hline 85 & Thalassarche melanophris & - & - & - & A & A & - \\
\hline 86 & Daption capense & - & - & A & A & $\mathrm{A}$ & - \\
\hline 87 & Ardenna grisea & - & - & A & A & A & - \\
\hline 88 & Calonectris diomedea & $\mathrm{RB}$ sc & RB vc & $\mathrm{M}, \mathrm{SB} \mathrm{c}$ & $\mathrm{SB}, \mathrm{M} \mathrm{sc}, \mathrm{W} \mathrm{r}$ & $\mathrm{SB}, \mathrm{M}, \mathrm{sc}, \mathrm{W}$ r & dec \\
\hline 89 & Calonectris borealis & - & - & - & - & A & - \\
\hline 90 & Puffinus yelkouan & $\mathrm{RB} \mathrm{r}$ & RB sc & SB sc & SB sc, W r & $\mathrm{SB}, \mathrm{M}, \mathrm{W} \mathrm{r}$ & st \\
\hline 91 & Puffinus mauretanicus & - & - & - & $\mathrm{A}$ & $\mathrm{A}$ & - \\
\hline 92 & Puffinus lherminieri baroli & - & - & - & A & $\mathrm{A}$ & - \\
\hline 93 & Bulweria bulwerii & - & - & - & A & A & - \\
\hline 94 & Ciconia nigra & $\mathrm{Mr}$ & $\mathrm{Mr}$ & M sc & M sc, W occ & M sc, W occ & flu \\
\hline 95 & Ciconia ciconia & $\mathrm{Mr}$ & $\mathrm{Mr}$ & M sc & $\mathrm{M} \mathrm{c}, \mathrm{W}, \mathrm{RB} \mathrm{sc}$ & $\mathrm{Mc}, \mathrm{W}, \mathrm{RB} \mathrm{sc}$ & vin \\
\hline 96 & Platalea leucorodia & M sc & $\mathrm{M}, \mathrm{W}$ sc & $\mathrm{M}, \mathrm{W}$ sc & $\mathrm{M}, \mathrm{W}$ sc, B? & $\mathrm{M}, \mathrm{W} \mathrm{c}$ & in \\
\hline 97 & Plegadis falcinellus & $\mathrm{Mc}, \mathrm{B} \mathrm{r}$ & $\mathrm{Mc}$ & M sc & $\mathrm{M}, \mathrm{W}$ sc, B r & Msc, W irr, B occ & flu \\
\hline 98 & Botaurus stellaris & M sc & M, RB (?) sc & $\mathrm{M}, \mathrm{W}$ sc & $\mathrm{M}, \mathrm{W}$ sc & M sc, W irr & st \\
\hline 99 & Ixobrychus minutus & M, SB vc & $\mathrm{M}, \mathrm{SB} \mathrm{c}$ & $\mathrm{M}, \mathrm{SB}$ sc & $\mathrm{M} \mathrm{sc}, \mathrm{Wr}, \mathrm{SB} \mathrm{sc}$ & $\mathrm{SB}, \mathrm{M} \mathrm{sc}, \mathrm{W}$ occ & vdec \\
\hline 100 & Nycticorax nycticorax & $\mathrm{M} \mathrm{c}, \mathrm{B} \mathrm{r}$ & $\mathrm{Mc}$ & $\mathrm{M} \mathrm{c}, \mathrm{B} \mathrm{r}$ & $\mathrm{M}, \mathrm{W}$ irr, B r & $\mathrm{B}, \mathrm{M} \mathrm{sc}, \mathrm{W}$ occ & dec \\
\hline 101 & Ardeola ralloides & $\mathrm{M} \mathrm{c}$ & $\mathrm{Mc}$ & M sc & $\mathrm{M} \mathrm{sc}, \mathrm{B} \mathrm{sc}$ & B, M sc, W irr & dec \\
\hline 102 & Bubulcus ibis & A & - & $M$ irr $r$ & $\mathrm{M}, \mathrm{W}$ sc, B r & $\mathrm{RB}, \mathrm{M}, \mathrm{W} \mathrm{c}$ & vin \\
\hline 103 & Ardea cinerea & $\mathrm{M}, \mathrm{W} \mathrm{c}, \mathrm{B} \mathrm{r}$ & $\mathrm{M}, \mathrm{W}$ c, B? & $\mathrm{M}, \mathrm{W}$ c, B r & $\mathrm{M}, \mathrm{W} \mathrm{c}, \mathrm{B} \mathrm{r}$ & $\mathrm{M}, \mathrm{W} \mathrm{c}, \mathrm{B} \mathrm{sc}$ & st \\
\hline 104 & Ardea purpurea & $\mathrm{M}, \mathrm{W} \mathrm{c}, \mathrm{B}$ sc & $\mathrm{M}, \mathrm{W} \mathrm{c}, \mathrm{B}$ ? & M sc & $\mathrm{M}, \mathrm{W}$ sc, B r & $\mathrm{M} \mathrm{sc}, \mathrm{B} \mathrm{r}$ & dec \\
\hline 105 & Ardea alba & $\mathrm{Mr}$ & M sc & M sc & $\mathrm{M}, \mathrm{W}$ sc & $\mathrm{M}, \mathrm{W}$ sc & in \\
\hline 106 & Egretta garzetta & M vc, W sc, B sc & $\mathrm{Mc}$ & $\mathrm{M} \mathrm{c}, \mathrm{W} \mathrm{r}$ & $\mathrm{M}, \mathrm{W} \mathrm{c}, \mathrm{B} \mathrm{r}$ & M, W c, B sc & dec \\
\hline $107 \mathrm{a}$ & Egretta gularis gularis & - & - & $\mathrm{A}$ & A & $\mathrm{A}$ & - \\
\hline $107 \mathrm{~b}$ & Egretta gularis schistacea & - & - & $\mathrm{A}$ & $\mathrm{A}$ & $\mathrm{A}$ & - \\
\hline 108 & Pelecanus crispus & A & A & A & A & A & - \\
\hline 109 & Pelecanus onocrotalus & A & A & A & $\mathrm{A}$ & M irr & - \\
\hline 110 & Morus bassanus & A & M irr? & $\mathrm{M}, \mathrm{W} \mathrm{sc}$ & $\mathrm{M}, \mathrm{W} \mathrm{sc}$ & $\mathrm{M}, \mathrm{W} \mathrm{sc}$ & vin \\
\hline 111 & Sula leucogaster & - & - & - & A & A & - \\
\hline 112 & Microcarbo pygmaeus & - & $\mathrm{A}$ & A & $\mathrm{A}$ & $\mathrm{A}$ & - \\
\hline 113 & Gulosus aristotelis desmarestii & $\mathrm{Mr}$ & RB vc & $\begin{array}{c}\text { RB r (only } \\
\text { Lampedusa), } \\
\text { M vr }\end{array}$ & $\mathrm{M}, \mathrm{W}$ irr, RB r & B Ex, M irr & vdec \\
\hline 114 & Phalacrocorax carbo & $\mathrm{M}, \mathrm{W}$ vc, B sc & $\mathrm{RB} v \mathrm{vc}$ & $\mathrm{M}, \mathrm{W} \mathrm{sc}$ & $\mathrm{M}, \mathrm{W}$ c, B occ & $\mathrm{M}, \mathrm{W} \mathrm{c}, \mathrm{B} \mathrm{r}$ & flu \\
\hline $115 \mathrm{a}$ & Burhinus oedicnemus oedicnemus & $\mathrm{M}, \mathrm{W} \mathrm{c}, \mathrm{B} \mathrm{sc}$ & $\mathrm{M}, \mathrm{RB} \mathrm{sc}$ & $\mathrm{RB}, \mathrm{M}, \mathrm{W}$ sc & RB r, M, W sc & $\mathrm{RB}, \mathrm{M}, \mathrm{W} \mathrm{sc}$ & st \\
\hline
\end{tabular}




\begin{tabular}{|c|c|c|c|c|c|c|c|}
\hline $115 b$ & Burhinus oedicnemus saharae & - & - & A & A & A & - \\
\hline 116 & Haematopus ostralegus & M sc & M irr & M sc & M sc, W irr & M sc, W irr & st \\
\hline 117 & Recurvirostra avosetta & $\mathrm{Mr}, \mathrm{B} \mathrm{r}$ & M irr & $\mathrm{M} \mathrm{sc}, \mathrm{B} \mathrm{r}$ & SB, M, W sc, & $\mathrm{SB}, \mathrm{M}, \mathrm{W}$ sc & in \\
\hline 118 & Himantopus himantopus & M sc & $\mathrm{M}$ & $\begin{array}{l}\text { M, B sc (first } \\
\text { breed. 1970s) }\end{array}$ & $\mathrm{SB}, \mathrm{M}, \mathrm{W}$ sc & $\mathrm{SB}, \mathrm{M}, \mathrm{W}$ sc & in \\
\hline 119 & Pluvialis squatarola & M sc & M sc & $\mathrm{M}, \mathrm{W} \mathrm{sc}$ & $\mathrm{M}, \mathrm{W} \mathrm{sc}$ & $\mathrm{M}, \mathrm{W} \mathrm{sc}$ & st \\
\hline $120 \mathrm{a}$ & Pluvialis apricaria apricaria & $\mathrm{M}, \mathrm{W}$ c & $\mathrm{M}, \mathrm{W}$ ve & $\mathrm{M}, \mathrm{W} \mathrm{c}$ & $\mathrm{M}, \mathrm{W} \mathrm{c}$ & $\mathrm{M}, \mathrm{W}$ sc & dec \\
\hline $120 \mathrm{~b}$ & Pluvialis apricaria altifrons & - & - & $\mathrm{M}, \mathrm{W} \mathrm{c}$ & $\mathrm{M}, \mathrm{W} \mathrm{c}$ & $\mathrm{M}, \mathrm{W}$ sc & - \\
\hline 121 & Pluvialis dominica & - & - & $\mathrm{A}$ & A & $\mathrm{A}$ & - \\
\hline 122 & Eudromias morinellus & $\mathrm{M}, \mathrm{W}$ sc & $\mathrm{M}, \mathrm{W} \mathrm{sc}$ & M sc & M r, W irr & $\mathrm{Mr}$ & dec \\
\hline 123 & Charadrius hiaticula & M, W c, B r (?) & $\mathrm{M}, \mathrm{W} \mathrm{sc}$ & $\mathrm{M}, \mathrm{W} \mathrm{sc}$ & $\mathrm{M} \mathrm{sc}, \mathrm{W} \mathrm{r}$ & $\mathrm{M}, \mathrm{W}$ sc & flu \\
\hline 124 & Charadrius dubius & $\mathrm{M}, \mathrm{W}$ sc, B r & M sc & B sc, M, W c & RB sc, Mc, W irr & RB sc, Mc, W irr & st \\
\hline 125 & Charadrius alexandrinus & $\mathrm{Mr}$ & $\mathrm{M}, \mathrm{B} \mathrm{sc}$ & $\mathrm{M}, \mathrm{W}, \mathrm{B} \mathrm{c}$ & $\mathrm{RB} \mathrm{c}, \mathrm{M}, \mathrm{W}$ sc & RB, M, W sc & flu \\
\hline 126 & Charadrius leschenaulti & - & - & - & A & A & - \\
\hline 127 & Charadrius asiaticus & - & - & A & A & A & - \\
\hline 128 & Vanellus vanellus & $\mathrm{M}, \mathrm{W}$ ve & $\mathrm{M}, \mathrm{W}$ ve & $\mathrm{M}, \mathrm{W} \mathrm{c}$ & $\mathrm{M}, \mathrm{W} \mathrm{c}$ & $\mathrm{M}, \mathrm{W}$ sc & vdec \\
\hline 129 & Vanellus gregarius & - & - & A & A & A & - \\
\hline 130 & Vanellus leucurus & - & - & A & A & A & - \\
\hline 131 & Bartramia longicauda & - & - & A & A & A & - \\
\hline 132 & Numenius phaeopus & $\mathrm{Mr}$ & $\mathrm{M}, \mathrm{W} \mathrm{sc}$ & M sc & M sc, W irr & M sc, W occ & in \\
\hline 133 & Numenius tenuirostris & $\mathrm{M} \mathrm{vc}$ & $\mathrm{M}, \mathrm{W} \mathrm{c}$ & A & A & Ex? & $\begin{array}{l}\text { vdec } \\
\text { (Ex?) }\end{array}$ \\
\hline $134 \mathrm{a}$ & Numenius arquata arquata & $\mathrm{M}, \mathrm{W} \mathrm{c}$ & $\mathrm{M}, \mathrm{W} \mathrm{c}$ & $\mathrm{M}, \mathrm{W} \mathrm{c}$ & $\mathrm{M}, \mathrm{W} \mathrm{sc}$ & $\mathrm{M}, \mathrm{W} \mathrm{sc}$ & dec \\
\hline $134 \mathrm{~b}$ & Numenius arquata orientalis & - & - & - & $\mathrm{M}, \mathrm{W} \mathrm{sc}$ & $\mathrm{M}, \mathrm{W} \mathrm{sc}$ & - \\
\hline 135 & Limosa lapponica & M irr, $r$ & M irr, $r$ & M sc & M sc, W irr & M sc, W irr & in \\
\hline 136 & Limosa limosa & $\mathrm{Mc}$ & M sc & $\mathrm{Mc}$ & M sc, W irr & M sc, W irr & dec \\
\hline 137 & Arenaria interpres & $\mathrm{Mr}$ & M irr & M sc & M sc, W irr & $\mathrm{M} \mathrm{sc}, \mathrm{W} \mathrm{r}$ & in \\
\hline 138 & Calidris canutus & A & $\mathrm{M}$ vr & $\mathrm{M}$ vr & $\mathrm{Mr}$ & M r, W irr & in \\
\hline 139 & Calidris pugnax & $\mathrm{M}, \mathrm{W} \mathrm{c}$ & $\mathrm{M}, \mathrm{W} \mathrm{c}$ & $\mathrm{Mc}, \mathrm{W}$ occ & $\mathrm{Mc}, \mathrm{W} \mathrm{r}$ & $\mathrm{M} \mathrm{sc}, \mathrm{W} \mathrm{r}$ & dec \\
\hline 140 & Calidris falcinellus & A & A & A & $\mathrm{M}$ irr & M irr & - \\
\hline 141 & Calidris ferruginea & $\mathrm{M}, \mathrm{W} \mathrm{c}$ & M vc, W irr & $\mathrm{Mc}$ & $\mathrm{Mc}$ & $\mathrm{Mc}$ & st \\
\hline 142 & Calidris temminckii & $\mathrm{Mr}$ & - & M sc & $\mathrm{M} \mathrm{sc}, \mathrm{W} \mathrm{r}$ & M sc, W irr & st \\
\hline 143 & Calidris ruficollis & - & - & - & - & $\mathrm{A}$ & - \\
\hline 144 & Calidris alba & M irr, sc & $\mathrm{M}, \mathrm{W} \mathrm{c}$ & $\mathrm{M}, \mathrm{W}$ sc & $\mathrm{M}, \mathrm{W}$ sc & $\mathrm{M}, \mathrm{W}$ sc & st \\
\hline $145 \mathrm{a}$ & Calidris alpina alpina & $\mathrm{M}, \mathrm{W} \mathrm{c}$ & $\mathrm{M} \mathrm{vc}, \mathrm{W}$ irr & $\mathrm{M}, \mathrm{W} \mathrm{c}$ & $\mathrm{Mc}, \mathrm{W} \mathrm{sc}$ & $\mathrm{M}, \mathrm{W} \mathrm{c}$ & flu \\
\hline $145 b$ & Calidris alpina schinzii & - & - & M sc & M sc & M sc & - \\
\hline 146 & Calidris maritima & - & - & - & A & A & - \\
\hline 147 & Calidris minuta & M vc & $\mathrm{M} v \mathrm{vc}, \mathrm{W}$ irr & $\mathrm{M}, \mathrm{W} \mathrm{c}$ & $\mathrm{Mc}, \mathrm{W} \mathrm{sc}$ & $\mathrm{Mc}, \mathrm{W} \mathrm{sc}$ & dec \\
\hline 148 & Calidris subruficollis & - & - & A & $\mathrm{A}$ & A & - \\
\hline 149 & Calidris melanotos & - & - & A & A & A & - \\
\hline 150 & Limnodromus scolopaceus & - & - & - & A & A & - \\
\hline 151 & Scolopax rusticola & $\mathrm{M}, \mathrm{W}$ ve & $\mathrm{M}, \mathrm{W}$ ve & $\mathrm{M}, \mathrm{W} \mathrm{c}$ & $\mathrm{M}, \mathrm{W} \mathrm{c}$ & $\mathrm{M}, \mathrm{W} \mathrm{c}$ & dec \\
\hline 152 & Gallinago stenura & - & - & - & A & A & - \\
\hline 153 & Gallinago media & $\mathrm{Mc}$ & M sc & M sc & M irr & M irr & vdec \\
\hline 154 & Gallinago gallinago & $\mathrm{M}, \mathrm{W}$ ve & $\mathrm{M}, \mathrm{W} \mathrm{c}$ & $\mathrm{M}, \mathrm{W} \mathrm{c}$ & $\mathrm{M}, \mathrm{W}$ c & $\mathrm{M}, \mathrm{W}$ sc & vdec \\
\hline 155 & Lymnocryptes minimus & $\mathrm{M}, \mathrm{W}$ c & $\mathrm{M}, \mathrm{W} \mathrm{c}$ & $\mathrm{M}, \mathrm{W}$ sc & $\mathrm{M} \mathrm{sc}, \mathrm{W} \mathrm{r}$ & M, W irr & vdec \\
\hline 156 & Phalaropus lobatus & - & - & A & A & M irr & - \\
\hline 157 & Phalaropus fulicarius & - & A & A & $\mathrm{A}$ & A & - \\
\hline 158 & Xenus cinereus & - & - & M irr, $r$ & M r, W occ & M irr, W irr & - \\
\hline
\end{tabular}




\begin{tabular}{|c|c|c|c|c|c|c|c|}
\hline 159 & Actitis hypoleucos & $\mathrm{RB}, \mathrm{M}, \mathrm{W} \mathrm{c}$ & $\mathrm{M} \mathrm{vc}, \mathrm{RB} \mathrm{sc}$ & $\mathrm{M}, \mathrm{W} \mathrm{c}$ & $\mathrm{M}, \mathrm{W} \mathrm{c}$ & $\mathrm{M}, \mathrm{W}$ c, B occ & st \\
\hline 160 & Actitis macularius & - & - & - & $\mathrm{A}$ & $\mathrm{A}$ & - \\
\hline 161 & Tringa ochropus & $\mathrm{M}, \mathrm{W}$ ve & $\mathrm{M}, \mathrm{W} \mathrm{c}$ & M sc & M sc, W irr & $\mathrm{Mc}, \mathrm{Wr}$ & flu \\
\hline 162 & Tringa flavipes & - & - & - & A & A & - \\
\hline 163 & Tringa erythropus & M sc & $\mathrm{M}, \mathrm{W}$ sc & $\mathrm{M}, \mathrm{W}$ sc & $\mathrm{M} \mathrm{sc}, \mathrm{W} \mathrm{r}$ & $\mathrm{M}, \mathrm{W} \mathrm{sc}$ & st \\
\hline 164 & Tringa nebularia & $\mathrm{M} \mathrm{c}$ & M sc & M sc & $\mathrm{M}, \mathrm{W}$ sc & $\mathrm{M}, \mathrm{W}$ sc & dec \\
\hline 165 & Tringa totanus & $\mathrm{M}, \mathrm{W} \mathrm{c}$ & $\mathrm{M} \mathrm{c}$ & $\mathrm{Mc}$ & $\mathrm{Mc}, \mathrm{W}$ sc, B occ & $\mathrm{M}, \mathrm{W}$ c, B occ & st \\
\hline 166 & Tringa glareola & M sc & $\mathrm{M}, \mathrm{W} \mathrm{c}$ & M sc & M c, W irr & $\mathrm{M} \mathrm{c}, \mathrm{Wr}$ & flu \\
\hline 167 & Tringa stagnatilis & $\mathrm{Mr}$ & M sc & M sc & M sc, W irr & $\mathrm{Mr}, \mathrm{W}$ irr & flu \\
\hline 168 & Turnix sylvaticus & $\mathrm{RB} \mathrm{c}$ & Ex (ca. 1920) & Ex & Ex & Ex & Ex \\
\hline 169 & Cursorius cursor & A & $\mathrm{A}$ & M irr & A & M occ & - \\
\hline 170 & Glareola pratincola & $\mathrm{SB}, \mathrm{Mc}$ & $\mathrm{SB}, \mathrm{M} \mathrm{sc}$ & $\mathrm{SB}$ r, M sc, & $\mathrm{SB}$ r, M sc & $\mathrm{SB} \mathrm{r,} \mathrm{M} \mathrm{sc}$ & dec \\
\hline 171 & Glareola nordmanni & - & A & A & $\mathrm{A}$ & $\mathrm{A}$ & - \\
\hline 172 & Hydrocoloeus minutus & $\mathrm{M}, \mathrm{W}$ sc & M irr, W sc & M irr, W sc & $\mathrm{M}, \mathrm{W} \mathrm{r}$ & $\mathrm{M}, \mathrm{W}$ irr & dec \\
\hline 173 & Xema sabini & - & - & - & - & A & - \\
\hline 174 & Rissa tridactyla & $\mathrm{M}, \mathrm{W} \mathrm{r}$ & $\mathrm{M}, \mathrm{W}$ irr, sc & $\mathrm{M}, \mathrm{W}$ sc & M, W irr & M, W irr & flu \\
\hline 175 & Larus genei & $\mathrm{Mr}$ & $\mathrm{M}, \mathrm{W} \mathrm{r}$ & $\mathrm{M}, \mathrm{W}$ sc & $\mathrm{M}, \mathrm{W}$ sc, B occ & $\mathrm{M}, \mathrm{W}$ sc & in \\
\hline 176 & Larus ridibundus & $\mathrm{M}, \mathrm{W}$ vc & $\mathrm{M}, \mathrm{W} \mathrm{c}$ & M, W c, B occ & $\mathrm{M}, \mathrm{W} \mathrm{c}$ & $\mathrm{M}, \mathrm{W}$ sc & vdec \\
\hline 177 & Larus pipixcan & - & - & - & - & A & - \\
\hline 178 & Larus atricilla & - & - & - & $\mathrm{A}$ & $\mathrm{A}$ & - \\
\hline 179 & Larus ichthyaetus & - & - & - & $\mathrm{M}, \mathrm{W}$ irr & $\mathrm{M}, \mathrm{W}$ irr & - \\
\hline 180 & Larus melanocephalus & $\mathrm{M}, \mathrm{W} \mathrm{vc}$ & $\mathrm{M}, \mathrm{W} \mathrm{c}$ & $\mathrm{M}, \mathrm{W}$ sc & $\mathrm{M}, \mathrm{W} \mathrm{c}$ & $\mathrm{M}, \mathrm{W}$ sc & vdec \\
\hline 181 & Larus audouinii & A & $\mathrm{RB} r$ & $\mathrm{M}, \mathrm{W}$ sc & $\mathrm{M} \mathrm{sc}, \mathrm{W} \mathrm{r}$ & $\mathrm{SB}, \mathrm{M}, \mathrm{W} \mathrm{r}$ & in \\
\hline 182 & Larus canus & $\mathrm{M}, \mathrm{W}$ vc & $\mathrm{M}, \mathrm{W}$ sc & M, W irr & $\mathrm{M}, \mathrm{W} \mathrm{r}$ & M, W irr & vdec \\
\hline $183 \mathrm{a}$ & Larus fuscus fuscus & $\mathrm{M}, \mathrm{W} \mathrm{sc}$ & $\mathrm{M}, \mathrm{W} \mathrm{c}$ & $\mathrm{M}, \mathrm{W}$ sc & $\mathrm{M}, \mathrm{W}$ sc & $\mathrm{M}, \mathrm{W} \mathrm{r}$ & flu \\
\hline $183 b$ & Larus fuscus intermedius & - & - & $\mathrm{M}, \mathrm{W}$ sc & $\mathrm{M}, \mathrm{W} \mathrm{sc}$ & $\mathrm{M}, \mathrm{W} \mathrm{sc}$ & - \\
\hline $183 \mathrm{c}$ & Larus fuscus graellsii & - & - & - & $\mathrm{M}, \mathrm{W} \mathrm{sc}$ & $\mathrm{A}$ & - \\
\hline $183 d$ & Larus fuscus heuglini & - & - & - & A & A & - \\
\hline 184 & Larus argentatus & - & - & - & $\mathrm{A}$ & $\mathrm{A}$ & - \\
\hline 185 & Larus michahellis & $\mathrm{M}, \mathrm{W} \mathrm{c}, \mathrm{B} \mathrm{r}$ & $\mathrm{RB} \mathrm{c}$ & $\mathrm{RB}, \mathrm{M}, \mathrm{W} \mathrm{c}$ & $\mathrm{RB}, \mathrm{M}, \mathrm{W} \mathrm{c}$ & RB, M, W vc & vin \\
\hline 186 & Larus cachinnans & - & - & - & $\mathrm{M}, \mathrm{W} \mathrm{r}$ & $\mathrm{M}, \mathrm{W}$ irr & - \\
\hline 187 & Larus marinus & $\mathrm{M} \mathrm{vr}$ & $\mathrm{M} \mathrm{vr}$ & - & $\mathrm{A}$ & $\mathrm{A}$ & - \\
\hline 188 & Sternula albifrons & $\mathrm{M} \mathrm{c}, \mathrm{SB} \mathrm{r}$ & $\mathrm{M} \mathrm{vc}, \mathrm{SB} \mathrm{c}$ & $\mathrm{M}, \mathrm{SB}$ sc & $\mathrm{SB}, \mathrm{M} \mathrm{sc}$ & $\mathrm{SB}, \mathrm{M} \mathrm{sc}$ & flu \\
\hline 189 & Gelochelidon nilotica & M sc & M sc & M sc & Msc, Wirr, Bocc & M sc, W occ & st \\
\hline 190 & Hydroprogne caspia & A & M occ, $\mathrm{r}$ & $\mathrm{M}, \mathrm{W}$ sc & M sc, W irr & M sc, W occ & in \\
\hline 191 & Chlidonias hybrida & $\mathrm{Mr}$ & M occ, $r$ & M sc & M sc, W irr & M sc, W irr & st \\
\hline 192 & Chlidonias leucopterus & M sc & $\mathrm{Mc}$ & M sc & M sc, W occ & M sc, W occ & st \\
\hline 193 & Chlidonias niger & $\mathrm{M} \mathrm{vc}$ & $\mathrm{Mc}$ & M sc & M sc, W irr & M sc, W occ & $\operatorname{dec}$ \\
\hline 194 & Sterna hirundo & M vc, SB sc & M vc, SB sc & M irr, $r$ & $\mathrm{M} \mathrm{r}, \mathrm{W}$ occ & M sc, B irr & dec \\
\hline 195 & Sterna paradisaea & - & - & A & A & A & - \\
\hline 196 & Thalasseus bengalensis emigratus & A & A & A & $\mathrm{A}$ & $\mathrm{A}$ & - \\
\hline 197 & Thalasseus sandvicensis & $\mathrm{M}, \mathrm{W}$ sc & $\mathrm{M} \mathrm{c}, \mathrm{B}$ ? & $\mathrm{M}, \mathrm{W} \mathrm{c}$ & $\mathrm{M}, \mathrm{W}$ sc, B occ & $\mathrm{M}, \mathrm{W}$ sc & flu \\
\hline 198 & Stercorarius longicaudus & $\mathrm{M}, \mathrm{W} \mathrm{r}$ & - & A & A & A & - \\
\hline 199 & Stercorarius parasiticus & $\mathrm{M}, \mathrm{W} \mathrm{r}$ & - & $\mathrm{M}, \mathrm{W}$ sc & $\mathrm{M}, \mathrm{W}$ vr & $\mathrm{M}, \mathrm{W} \mathrm{r}$ & - \\
\hline 200 & Stercorarius pomarinus & $\mathrm{M}, \mathrm{W} \mathrm{r}$ & $\mathrm{M}, \mathrm{W} \mathrm{r}$ & $\mathrm{M}, \mathrm{W}$ sc & $\mathrm{M}, \mathrm{W}$ sc & $\mathrm{M}, \mathrm{W} \mathrm{r}$ & st \\
\hline
\end{tabular}




\begin{tabular}{|c|c|c|c|c|c|c|c|}
\hline 201 & Catharacta skua & - & - & $\mathrm{M}, \mathrm{W} \mathrm{r}$ & $\mathrm{M}, \mathrm{W}$ vr & $\mathrm{M}, \mathrm{W} \mathrm{r}$ & - \\
\hline 202 & Fratercula arctica & M irr, vr & M, W irr, sc & $\mathrm{M}, \mathrm{W} \mathrm{r}$ & $\mathrm{M}, \mathrm{W}$ irr & $\mathrm{M}, \mathrm{W}$ irr & in \\
\hline 203 & Alca torda & M irr, vr & M, W irr, sc & $\mathrm{M}, \mathrm{W}$ irr, $\mathrm{r}$ & M, W occ & $\mathrm{M}, \mathrm{W}$ irr & in \\
\hline 204 & Uria aalge & - & - & - & A & $\mathrm{A}$ & - \\
\hline 205 & Tyto alba & RB vc & $\mathrm{RB} s \mathrm{~s}$ & RB sc, M, W sc & $\mathrm{RB}$ sc, $\mathrm{M}, \mathrm{W}$ sc & RB sc, M, W sc & dec \\
\hline 206 & Athene noctua & $\mathrm{RB} \mathrm{c}$ & $\mathrm{RB} s \mathrm{c}$ & $\mathrm{RB} \mathrm{c}$ & $\mathrm{RB} \mathrm{c}$ & $\mathrm{RB} \mathrm{c}$ & flu \\
\hline 207 & Otus scops & $\mathrm{SB} \mathrm{c}, \mathrm{M}$ ve & $\mathrm{RB} \mathrm{c}, \mathrm{Mc}$ & $\mathrm{RB}, \mathrm{M} \mathrm{c}$ & $\mathrm{RB}, \mathrm{M} \mathrm{c}, \mathrm{W} \mathrm{sc}$ & $\mathrm{SB}, \mathrm{M}, \mathrm{W} \mathrm{c}$ & dec \\
\hline 208 & Asio otus & $\mathrm{RB} s \mathrm{~s}$ & RB c & $\mathrm{M}, \mathrm{RB} \mathrm{sc}$ & $\mathrm{RB}$ r, M, W sc & RB sc, M, W r & st \\
\hline 209 & Asio flammeus & $\mathrm{M}, \mathrm{W}, \mathrm{B}(?) \mathrm{sc}$ & $\mathrm{M}, \mathrm{W} \mathrm{c}$ & M sc & M sc, W irr & M sc, W irr & st \\
\hline 210 & Strix aluco & $\mathrm{RB} \mathrm{c}$ & $\mathrm{RB} \mathrm{c}$ & $\mathrm{RB} \mathrm{c}$ & $\mathrm{RB} \mathrm{c}$ & $\mathrm{RB} \mathrm{c}$ & st \\
\hline 211 & Bubo bubo & $\mathrm{RB} \mathrm{sc}$ & $\mathrm{RB} s \mathrm{c}$ & Ex (1970’) & Ex & Ex & Ex \\
\hline 212 & Pandion haliaetus & RB r & M, W sc, RB? & $\begin{array}{l}\text { M, W sc, B } \\
\text { (Ex 1960') }\end{array}$ & M sc, W irr & $\mathrm{M}, \mathrm{W}$ sc & dec \\
\hline 213 & Pernis apivorus & $\mathrm{M} \mathrm{c}$ & $\mathrm{Mc}$ & $\mathrm{M} \mathrm{c}$ & Mc, W irr, B occ & $\mathrm{M} \mathrm{c}, \mathrm{B}$ occ & st \\
\hline 214 & Gypaetus barbatus & $\mathrm{RB}$ vr & Ex (ca. 1850) & Ex & Ex, A? & Ex & Ex \\
\hline 215 & Neophron percnopterus & SB r & M, SB & $\begin{array}{l}\text { SB sc (20 } \\
\text { pairs) }\end{array}$ & $\begin{array}{c}\text { SB r (5-6 pairs), } \\
\text { M sc, W irr }\end{array}$ & $\begin{array}{c}\text { SB (7-9 pairs), } \\
\text { M r, W irr }\end{array}$ & dec \\
\hline 216 & Circaetus gallicus & M sc & M sc & $\mathrm{M}, \mathrm{W} \mathrm{sc}$ & M sc, W r, SB? & $\mathrm{M}, \mathrm{W}$ sc, SB? & st \\
\hline 217 & Gyps fulvus & $\mathrm{RB} \mathrm{c}$ & $\mathrm{RB} \mathrm{c}$ & $\begin{array}{c}\text { Ex (1965), M } \\
\text { irr }\end{array}$ & Ex, A? & $\mathrm{RB}$ reintr & $\begin{array}{l}\mathrm{RB} \\
\text { reintr }\end{array}$ \\
\hline 218 & Gyps rueppelli & - & - & - & - & $\mathrm{A}$ & - \\
\hline 219 & Aegypius monachus & A? & A? & A? & $\mathrm{A}$ & $\mathrm{A}$ & - \\
\hline 220 & Clanga pomarina & $\mathrm{RB}(?) \mathrm{r}$ & $\mathrm{RB}(?) \mathrm{r}$ & $\mathrm{Mr}$ & $\mathrm{M}, \mathrm{W}$ irr & $\mathrm{Mr}, \mathrm{W}$ irr & - \\
\hline 221 & Clanga clanga & - & - & M irr, $r$ & $\mathrm{M}, \mathrm{W}$ irr & $\mathrm{M}, \mathrm{W}$ occ & - \\
\hline 222 & Aquila nipalensis & - & - & - & A & $\mathrm{A}$ & - \\
\hline 223 & Aquila heliaca & - & - & A & A & A & - \\
\hline 224 & Aquila chrysaetos & $\mathrm{RB} \mathrm{r}$ & $\mathrm{RB} \mathrm{r}$ & $\underset{\text { pairs }}{\mathrm{RB} \mathrm{sc}(15}$ & $\mathrm{RB} \mathrm{sc}$ & $\begin{array}{c}\text { RB sc (17-21 } \\
\text { pairs) }\end{array}$ & st \\
\hline 225 & Aquila fasciata & $\mathrm{RB} \mathrm{r}$ & $\mathrm{RB} \mathrm{r}$ & $\begin{array}{c}\mathrm{RB}_{12} \mathrm{r} \text { (less thairs) } \\
\text { part }\end{array}$ & $\begin{array}{c}\text { RB r (less than } \\
20 \text { pairs) }\end{array}$ & $\begin{array}{c}\text { RB sc (ca. } 50 \\
\text { pairs) }\end{array}$ & vin \\
\hline 226 & Hieraaetus pennatus & - & - & M sc & $\mathrm{M} \mathrm{sc}, \mathrm{W} \mathrm{r}$ & $\mathrm{M}, \mathrm{W}$ sc & vin \\
\hline 227 & Circus aeruginosus & $\mathrm{RB} \mathrm{c}, \mathrm{M} \mathrm{c}$ & $\mathrm{RB} \mathrm{c}$ & $\mathrm{M}, \mathrm{W} \mathrm{c}$ & $\mathrm{M} \mathrm{c}, \mathrm{W} \mathrm{sc}$ & $\mathrm{M}, \mathrm{W} \mathrm{sc}$ & dec \\
\hline 228 & Circus cyaneus & $\mathrm{RB}(?) \mathrm{sc}$ & $\mathrm{RB}(?) \mathrm{sc}$ & $\mathrm{M}, \mathrm{W} \mathrm{sc}$ & $\mathrm{M} \mathrm{sc}, \mathrm{W} \mathrm{r}$ & $\mathrm{M} \mathrm{sc}, \mathrm{W} \mathrm{r}$ & dec \\
\hline 229 & Circus macrourus & $\mathrm{M} \mathrm{sc}$ & $\mathrm{M} \mathrm{sc}$ & M sc & M sc, W occ & M sc, W occ & st \\
\hline 230 & Circus pygargus & M sc & $\mathrm{M} \mathrm{sc}$ & $\mathrm{M} \mathrm{sc}$ & M sc, W occ & M sc & st \\
\hline 231 & Accipiter brevipes & - & - & - & A & A & - \\
\hline 232 & Accipiter nisus & $\mathrm{RB} \mathrm{c}$ & $\mathrm{RB}, \mathrm{Mc}$ & $\mathrm{M}, \mathrm{W}$ c, B sc & RB, M, W sc, & RB, M, W sc & st \\
\hline 233 & Accipiter gentilis & $\mathrm{RB} s \mathrm{c}$ & $\mathrm{RB} s \mathrm{c}$ & A & $\mathrm{A}$ & M irr & dec \\
\hline 234 & Haliaeetus albicilla & - & M occ & A & $\mathrm{A}$ & $\mathrm{A}$ & - \\
\hline 235 & Milvus milvus & $\mathrm{RB} \mathrm{c}$ & $\mathrm{RB}, \mathrm{M} \mathrm{c}$ & $\underset{\substack{\text { M sc, } \\
40 \text { pairs })}}{\text { (ca. }}$ & $\begin{array}{c}\text { M, } \mathrm{W} \text { r, RB } \\
\text { (5-6 pairs) }\end{array}$ & RB, M, W vr & vdec \\
\hline 236 & Milvus migrans & $\mathrm{M} v \mathrm{vr}$ & $\mathrm{Mr}$ & $\begin{array}{l}\text { M c, B sc (first } \\
\text { breed. 1979) }\end{array}$ & M c, $\underset{\text { sc }}{\text { W sc, B }}$ & $\begin{array}{l}\text { M c, W irr, } \\
\text { RB? }\end{array}$ & flu \\
\hline 237 & Buteo lagopus & - & - & A & A & A & - \\
\hline $238 \mathrm{a}$ & Buteo buteo buteo & $\mathrm{RB} \mathrm{c}$ & $\mathrm{RB} \mathrm{c}$ & $\mathrm{RB}, \mathrm{M}, \mathrm{W} \mathrm{c}$ & RB c, M, W sc & $\mathrm{RB} \mathrm{c}, \mathrm{M}, \mathrm{W}$ sc & st \\
\hline $238 \mathrm{~b}$ & Buteo buteo vulpinus & - & - & $\mathrm{M}$ vr & $\mathrm{Mr}$ & $\mathrm{Mr}$ & - \\
\hline $239 \mathrm{a}$ & Buteo rufinus rufinus & - & - & A & $\mathrm{Mr}, \mathrm{W}$ occ & $\mathrm{Mr}, \mathrm{W}$ occ & - \\
\hline $239 b$ & Buteo rufinus cirtensis & - & - & - & $\begin{array}{c}\text { M r, B occ } \\
\text { (Pantelleria) }\end{array}$ & $\begin{array}{l}\text { M irr, B occ } \\
\text { (Pantelleria) }\end{array}$ & in \\
\hline
\end{tabular}




\begin{tabular}{|c|c|c|c|c|c|c|c|}
\hline 240 & Upupa epops & $\mathrm{SB} \mathrm{c}, \mathrm{M} \mathrm{c}$ & $\mathrm{SB} \mathrm{c}, \mathrm{M} \mathrm{vc}$ & $\mathrm{SB}, \mathrm{M} \mathrm{c}, \mathrm{W} \mathrm{r}$ & $\mathrm{SB}, \mathrm{M} \mathrm{c}, \mathrm{W} \mathrm{r}$ & $\mathrm{SB}, \mathrm{M} \mathrm{c}, \mathrm{W} \mathrm{r}$ & st \\
\hline 241 & Merops persicus & $\mathrm{A}$ & $\mathrm{A}$ & $\mathrm{A}$ & A & $\mathrm{A}$ & - \\
\hline 242 & Merops apiaster & SB vr, M c & $\mathrm{SB} \mathrm{r,} \mathrm{M} \mathrm{c}$ & $\mathrm{M}, \mathrm{SB} \mathrm{r}$ & SB sc, M c & $\mathrm{SB}, \mathrm{M} \mathrm{c}$ & vin \\
\hline 243 & Coracias garrulus & SB c & SB c & $\mathrm{M}, \mathrm{SB}$ sc & $\mathrm{SB}, \mathrm{M} \mathrm{sc}$ & $\mathrm{SB}, \mathrm{M} \mathrm{sc}$ & dec \\
\hline 244 & Alcedo atthis & RB sc, M, W c & RB c & $\mathrm{M}, \mathrm{W}, \mathrm{RB} \mathrm{r}$ & $\mathrm{RB}, \mathrm{M}, \mathrm{W}$ sc & $\mathrm{RB}, \mathrm{M}, \mathrm{W}$ sc & dec \\
\hline 245 & Jynx torquilla & $\mathrm{Mc}$ & $\mathrm{M}, \mathrm{W}$ vc, B? & $\mathrm{M}, \mathrm{W}$ sc, B r & SB sc, M c, W r & $\mathrm{SB}, \mathrm{M}, \mathrm{W}$ sc & dec \\
\hline 246 & Picus viridis & $\mathrm{RB} v \mathrm{vr}$ & $\mathrm{RB} v \mathrm{vr}$ & Ex (ca. 1930) & Ex & Ex & Ex \\
\hline 247 & Dryocopus martius & $\mathrm{RB} v \mathrm{vr}$ & $\mathrm{RB} ?$ & Ex (1900?) & Ex & $\mathrm{A}$ & Ex \\
\hline 248 & Dryobates minor & $\mathrm{RB} \mathrm{r}$ & $\mathrm{RB} \mathrm{r}$ & Ex (ca. 1930) & Ex & Ex & Ex \\
\hline 249 & Dendrocopos major & RB vc & $\mathrm{RB}$ sc & $\mathrm{RB} \mathrm{c}$ & $\mathrm{RB} \mathrm{c}$ & RB c & st \\
\hline 250 & Falco naumanni & $\mathrm{SB}$ vc, W & $\mathrm{RB} v \mathrm{c}$ & $\mathrm{M} \mathrm{c}, \mathrm{SB} \mathrm{sc}$ & SBc,Msc, Wocc & $\mathrm{SB}, \mathrm{M} \mathrm{sc}, \mathrm{W}$ irr & vdec \\
\hline 251 & Falco tinnunculus & $\mathrm{RB} \mathrm{vc}$ & $\mathrm{RB} v \mathrm{vc}$ & RB vc, M, W c & $\mathrm{RB}, \mathrm{M}, \mathrm{W} \mathrm{c}$ & $\mathrm{RB}, \mathrm{M}, \mathrm{W} \mathrm{c}$ & dec \\
\hline 252 & Falco vespertinus & $\mathrm{Mc}$ & $\mathrm{Mc}$ & $\mathrm{Mc}$ & M sc & M sc & dec \\
\hline 253 & Falco amurensis & - & - & - & A & $\mathrm{A}$ & - \\
\hline 254 & Falco eleonorae & - & M occ & $\begin{array}{c}\text { M, SB sc (ca. } \\
150 \text { pairs) }\end{array}$ & SB sc, $\mathrm{M} \mathrm{r}$ & $\mathrm{SB}, \mathrm{M} \mathrm{sc}$ & in \\
\hline 255 & Falco concolor & - & - & - & $\mathrm{A}$ & $\mathrm{A}$ & - \\
\hline 256 & Falco columbarius & $\mathrm{M}, \mathrm{W} \mathrm{sc}$ & M irr & $\mathrm{M}, \mathrm{W} \mathrm{sc}$ & $\mathrm{M}, \mathrm{W} \mathrm{sc}$ & $\mathrm{M}, \mathrm{W} \mathrm{r}$ & flu \\
\hline 257 & Falco subbuteo & $\mathrm{M} \mathrm{c}, \mathrm{B}$ ? & M, SB sc & $\mathrm{M}, \mathrm{c}, \mathrm{SB}$ sc & $\mathrm{SB}, \mathrm{Mc}, \mathrm{W}$ occ & $\mathrm{SB}, \mathrm{M} \mathrm{sc}$ & dec \\
\hline $258 \mathrm{a}$ & Falco biarmicus feldeggii & - & $\mathrm{A}$ & $\begin{array}{c}\text { RB sc (ca. } 60 \\
\text { pairs) }\end{array}$ & $\begin{array}{c}\mathrm{RB}(100-120 \\
\text { pairs })\end{array}$ & $\begin{array}{c}\text { RB (ca. } 60 \\
\text { pairs) }\end{array}$ & flu \\
\hline $258 \mathrm{~b}$ & Falco biarmicus erlangeri & - & - & - & $\mathrm{A}$ & A & - \\
\hline 259 & Falco cherrug & A & A & $\mathrm{A}$ & $\mathrm{M}, \mathrm{W} \mathrm{r}$ & $\mathrm{M}, \mathrm{W} \mathrm{r}$ & in \\
\hline $260 \mathrm{a}$ & Falco peregrinus calidus & - & - & $\mathrm{M}, \mathrm{W} \mathrm{r}$ & $\mathrm{M}, \mathrm{W} \mathrm{r}$ & $\mathrm{M}, \mathrm{W} \mathrm{r}$ & - \\
\hline $260 \mathrm{~b}$ & Falco peregrinus peregrinus & - & - & $\mathrm{M}, \mathrm{W} \mathrm{r}$ & $\mathrm{M}, \mathrm{W} \mathrm{r}$ & $\mathrm{M}, \mathrm{W} \mathrm{r}$ & - \\
\hline $260 \mathrm{c}$ & Falco peregrinus brookei & $\mathrm{RB} \mathrm{sc}$ & $\mathrm{RB}, \mathrm{M} \mathrm{c}$ & $\begin{array}{l}\mathrm{RB} \text { (ca. } 150 \\
\text { pairs) }\end{array}$ & $\begin{array}{c}\mathrm{RB} \text { (ca. } 250 \\
\text { pairs) }\end{array}$ & $\begin{array}{c}\text { RB (ca. } 300 \\
\text { pairs) }\end{array}$ & vin \\
\hline $260 \mathrm{~d}$ & Falco peregrinus pelegrinoides & - & - & A & A & $\mathrm{A}$ & - \\
\hline 261 & Myiopsitta monachus & - & - & - & $\mathrm{RB} \mathrm{r}$, al & $\mathrm{RB} \mathrm{r}$, al & st \\
\hline 262 & Psittacula krameri & - & - & - & RB sc, al & RB sc, al & in \\
\hline 263 & Oriolus oriolus & $\mathrm{M} \mathrm{vc}, \mathrm{B} \mathrm{r}$ & SB sc, M, vc & M, SB sc & $\mathrm{SB} \mathrm{sc}, \mathrm{M} \mathrm{c}$ & $\mathrm{SB}, \mathrm{M} \mathrm{sc}$ & vdec \\
\hline 264 & Lanius collurio & SB sc & $\mathrm{M} \mathrm{sc}$ & $\mathrm{M} \mathrm{sc}, \mathrm{SB} \mathrm{r}$ & $\mathrm{SB}$ r, M sc & $\mathrm{SB}$ r, M sc & dec \\
\hline 265 & Lanius isabellinus & - & - & - & $\mathrm{A}$ & $\mathrm{A}$ & - \\
\hline 266 & Lanius minor & SB vc & $\mathrm{SB}, \mathrm{M} v \mathrm{v}$ & $\mathrm{M} \mathrm{sc}, \mathrm{SB} \mathrm{r}$ & $\mathrm{SB} \mathrm{r,M} \mathrm{r}$ & $\mathrm{B}(\mathrm{Ex} ?), \mathrm{M} \mathrm{r}$ & vdec \\
\hline $267 \mathrm{a}$ & Lanius excubitor excubitor & A & A & A & A & A & - \\
\hline $267 \mathrm{~b}$ & Lanius excubitor algeriensis & - & - & - & - & A & - \\
\hline $267 \mathrm{c}$ & Lanius excubitor elegans & - & - & A & $\mathrm{A}$ & $\mathrm{A}$ & - \\
\hline $267 d$ & Lanius excubitor pallidirostris & - & - & A & $\mathrm{A}$ & $\mathrm{A}$ & - \\
\hline 268 & Lanius meridionalis & - & - & - & A & A & - \\
\hline $269 \mathrm{a}$ & Lanius senator senator & SB c & $\mathrm{SB}, \mathrm{M} \mathrm{vc}$ & $\mathrm{M} \mathrm{sc}, \mathrm{SB}$ r & SB sc, M c & SB r, M sc & vdec \\
\hline $269 b$ & Lanius senator niloticus & - & - & $\mathrm{A}$ & $\mathrm{A}$ & $\mathrm{A}$ & - \\
\hline $269 \mathrm{c}$ & Lanius senator badius & - & - & $\mathrm{Mr}$ & M sc, SB occ & $\mathrm{M} \mathrm{r}, \mathrm{SB}$ occ & - \\
\hline 270 & Pyrrhocorax pyrrhocorax & $\mathrm{RB} \mathrm{c}$ & $\mathrm{RB} \mathrm{c}$ & $\mathrm{RB} \mathrm{sc}$ & $\mathrm{RB} \mathrm{sc}$ & $\mathrm{RB} \mathrm{sc}$ & dec \\
\hline 271 & Garrulus glandarius & RB c & $\mathrm{RB} \mathrm{c}$ & $\mathrm{RB} \mathrm{c}$ & $\mathrm{RB} \mathrm{c}$ & RB c & st \\
\hline 272 & Pica pica & RB c & RB c & $\mathrm{RB} \mathrm{c}$ & RB vc & $\mathrm{RB} \mathrm{c}$ & st \\
\hline 273 & Nucifraga caryocatactes & A? & A? & - & - & - & - \\
\hline 274 & Corvus monedula & RB c & RB c & $\mathrm{RB} \mathrm{c}$ & RB c & RB c & st \\
\hline
\end{tabular}




\begin{tabular}{|c|c|c|c|c|c|c|c|}
\hline 275 & Corvus frugilegus & $\mathrm{M}, \mathrm{W} \mathrm{c}$ & $\mathrm{M}, \mathrm{W} \mathrm{c}$ & A & A & A & vdec \\
\hline $276 \mathrm{a}$ & Corvus corax corax & $\mathrm{RB} \mathrm{vc}$ & $\mathrm{RB} \mathrm{c}$ & $\mathrm{RB} \mathrm{c}$ & $\mathrm{RB} \mathrm{c}$ & $\mathrm{RB} \mathrm{sc}$ & vdec \\
\hline $276 b$ & Corvus corax tingitanus & - & - & - & - & A (Linosa) & - \\
\hline 277 & Corvus ruficollis & - & - & - & - & A & - \\
\hline 278 & Corvus corone cornix & RB c & RB c & RB c & $\mathrm{RB} v \mathrm{c}$ & $\mathrm{RB} v \mathrm{v}$ & in \\
\hline 279 & Periparus ater & $\mathrm{RB} v \mathrm{vr}$ & M, W sc & RB, M, W sc & $\underset{\text { irr }}{\mathrm{RB} \text { sc, } \mathrm{M}, \mathrm{W}}$ & $\begin{array}{l}\text { RB sc, } M \text { irr, } \\
\text { W irr }\end{array}$ & in \\
\hline 280 & Poecile palustris & M irr & $\mathrm{M}, \mathrm{W}, \mathrm{RB} ? \mathrm{r}$ & $\mathrm{RB} \mathrm{r}$ & $\mathrm{RB} \mathrm{r}$ & $\mathrm{RB} v \mathrm{vr}$ & st \\
\hline 281 & Cyanistes caeruleus & RB c & $\mathrm{RB}, \mathrm{M} \mathrm{c}$ & $\mathrm{RB} \mathrm{c}$ & $\mathrm{RB} \mathrm{c}$ & $\mathrm{RB} \mathrm{c}$ & st \\
\hline 282 & $\begin{array}{l}\text { Cyanistes teneriffae } \\
\text { ultramarinus }\end{array}$ & - & - & $\begin{array}{l}\text { RB c (only } \\
\text { Pantelleria) }\end{array}$ & $\begin{array}{l}\text { RB c (only } \\
\text { Pantelleria) }\end{array}$ & $\begin{array}{l}\text { RB c (only } \\
\text { Pantelleria) }\end{array}$ & st \\
\hline 283 & Parus major & $\mathrm{RB} v \mathrm{v}$ & RB vc & RB vc & $\mathrm{RB} v \mathrm{c}$ & $\mathrm{RB} v \mathrm{c}$ & st \\
\hline 284 & Remiz pendulinus & $\mathrm{RB} \mathrm{c}$ & $\mathrm{RB}, \mathrm{M} \mathrm{sc}$ & $\mathrm{RB} s \mathrm{c}$ & $\mathrm{RB}, \mathrm{M}, \mathrm{W}$ sc & RB, M, W sc & dec \\
\hline 285 & Ammomanes cinctura & - & - & A & A & A & - \\
\hline 286 & Alaudala rufescens & - & - & A & A & A & - \\
\hline 287 & Melanocorypha bimaculata & - & - & A & A & A & - \\
\hline 288 & Melanocorypha calandra & RB vc, M sc & $\mathrm{RB} v \mathrm{c}$ & $\mathrm{RB} s \mathrm{c}$ & RB sc, Mr, Wr & $\mathrm{RB}, \mathrm{M} \mathrm{r}, \mathrm{W} \mathrm{r}$ & vdec \\
\hline 289 & Calandrella brachydactyla & SB c & $\mathrm{SB} \mathrm{c}$ & $\mathrm{M} \mathrm{c}, \mathrm{SB} \mathrm{sc}$ & $\mathrm{SB}, \mathrm{M} \mathrm{sc}$ & $\mathrm{SB}, \mathrm{M} \mathrm{sc}$ & dec \\
\hline 290 & Eremophila alpestris & - & - & A & A & A & - \\
\hline 291 & Lullula arborea & $\mathrm{M}, \mathrm{W}, \mathrm{B} \mathrm{sc}$ & $\mathrm{M}, \mathrm{W}, \mathrm{B}$ sc & $\mathrm{M}, \mathrm{W}, \mathrm{RB}$ sc & $\mathrm{RB}, \mathrm{M}, \mathrm{W}$ sc & RB, M, W sc & st \\
\hline 292 & Alauda arvensis & M, W vc, B sc & M, W vc, B sc & $\mathrm{M}, \mathrm{W} \mathrm{c}, \mathrm{RB} \mathrm{sc}$ & RB r, M, W c & $\mathrm{RB}$ r, M, W sc & vdec \\
\hline 293 & Galerida cristata & $\mathrm{RB} v \mathrm{c}$ & $\mathrm{RB} v \mathrm{c}$ & $\mathrm{RB} \mathrm{c}$ & $\mathrm{RB} \mathrm{c}$ & $\mathrm{RB} \mathrm{c}$ & dec \\
\hline 294 & Panurus biarmicus & $\mathrm{RB} \mathrm{r}$ & $\mathrm{RB} r$ & $\begin{array}{c}\text { B Ex (ca. } \\
1950), \text { M occ }\end{array}$ & A & A & Ex \\
\hline $295 \mathrm{a}$ & Cisticola juncidis cisticola & - & - & $\begin{array}{c}\mathrm{RB} \mathrm{c} \\
\text { (Pantelleria) }\end{array}$ & $\begin{array}{c}\text { RB c } \\
\text { (Pantelleria, } \\
\text { Lampedusa) }\end{array}$ & $\begin{array}{c}\text { RB c } \\
\text { (Pantelleria, } \\
\text { Lampedusa) }\end{array}$ & in \\
\hline $295 b$ & Cisticola juncidis juncidis & $\mathrm{M}, \mathrm{W}$ vc, B c & $\mathrm{RB}, \mathrm{M} \mathrm{c}$ & RB c & RB c & $\mathrm{RB} \mathrm{c}$ & st \\
\hline 296 & Iduna pallida & - & - & - & A & A & - \\
\hline 297 & Iduna opaca & - & - & - & A & A & - \\
\hline 298 & Hippolais polyglotta & A & M sc & $\mathrm{M} \mathrm{r,} \mathrm{B} \mathrm{occ}$ & M r, B occ & $\mathrm{Mr}$ & flu \\
\hline 299 & Hippolais icterina & M c, B (?) & M vc, B (?) & M sc & $\mathrm{Mc}$ & $\mathrm{Mc}$ & flu \\
\hline 300 & Acrocephalus paludicola & $\mathrm{Mr}$ & M, B (?) & A & A & M irr & dec \\
\hline 301 & Acrocephalus melanopogon & $\mathrm{Mr}$ & A & $\mathrm{M}, \mathrm{W}$ sc, B? & $\mathrm{M}, \mathrm{W}$ sc, B? & $\mathrm{M}, \mathrm{W} \mathrm{r}$ & flu \\
\hline 302 & Acrocephalus schoenobaenus & $\mathrm{Mc}$ & $\mathrm{Mc}$ & $\mathrm{M} \mathrm{sc}$ & $\mathrm{M} \mathrm{sc}$ & M sc & dec \\
\hline 303 & Acrocephalus palustris & - & $\mathrm{Mr}$ & A & A & M vr & - \\
\hline 304 & Acrocephalus scirpaceus & M, SB c & M, SB c & M, SB c & $\mathrm{SB}$ sc, $\mathrm{M} \mathrm{c}, \mathrm{W}$ ? & $\mathrm{SB}, \mathrm{M} \mathrm{c}$ & st \\
\hline 305 & Acrocephalus agricola & - & - & - & A & A & - \\
\hline 306 & Acrocephalus arundinaceus & M, SB c & M, SB c & M, SB c & $\mathrm{SB}, \mathrm{M} \mathrm{sc}$ & $\mathrm{SB}, \mathrm{M} \mathrm{sc}, \mathrm{W}$ occ & st \\
\hline 307 & Locustella luscinioides & $\mathrm{M}$ vr & A? & A or M irr & A & M irr & - \\
\hline 308 & Locustella naevia & A & A? & A & A & $\mathrm{M}$ irr & - \\
\hline 309 & Delichon urbicum & SB vc, W c & $\mathrm{SB}$ ve & M, SB c & $\mathrm{SB}, \mathrm{M} \mathrm{c}, \mathrm{W}$ irr & $\mathrm{SB}, \mathrm{M} \mathrm{c}, \mathrm{W}$ irr & dec \\
\hline 310 & Cecropis daurica & $\mathrm{Mr}$ & M irr & $\mathrm{M}, \mathrm{SB} \mathrm{r}$ & $\mathrm{SB}, \mathrm{M} \mathrm{sc}, \mathrm{W}$ occ & $\mathrm{SBr}, \mathrm{Msc}, \mathrm{W}$ occ & in \\
\hline 311 & Hirundo rustica & $\mathrm{M} \mathrm{vc}, \mathrm{B} \mathrm{sc}$ & M, SB c & M, SB c & $\mathrm{SB}$ c, M vc, Wr & $\mathrm{SB}, \mathrm{M} \mathrm{c}, \mathrm{W}$ irr & dec \\
\hline 312 & Ptyonoprogne rupestris & $\mathrm{RB} \mathrm{c}$ & $\mathrm{RB}, \mathrm{M} \mathrm{c}$ & $\mathrm{RB}, \mathrm{M}, \mathrm{W}$ sc & $\mathrm{RB}, \mathrm{M}, \mathrm{W} \mathrm{sc}$ & $\mathrm{RB}, \mathrm{M}, \mathrm{W}$ sc & dec \\
\hline 313 & Riparia riparia & $\mathrm{Mc}$ & M vc & $\mathrm{Mc}$ & $\mathrm{Mc}, \mathrm{W}$ irr & M sc, W occ & dec \\
\hline 314 & Phylloscopus orientalis & - & - & - & A & A & - \\
\hline 315 & Phylloscopus bonelli & M sc & M sc & M sc & $\mathrm{Mr}$ & $\mathrm{Mr}$ & dec \\
\hline 316 & Phylloscopus sibilatrix & M c, B r (?) & M c, B (?) & $\mathrm{Mc}$ & $\mathrm{Mc}$ & $\mathrm{Mc}$ & st \\
\hline
\end{tabular}




\begin{tabular}{|c|c|c|c|c|c|c|c|}
\hline 317 & Phylloscopus inornatus & - & - & $\mathrm{A}$ & $\mathrm{A}$ & M sc, W occ & - \\
\hline 318 & Phylloscopus humei & - & - & - & A & A & - \\
\hline 319 & Phylloscopus proregulus & - & - & - & $\mathrm{A}$ & A & - \\
\hline 320 & Phylloscopus fuscatus & - & - & - & $\mathrm{A}$ & A & - \\
\hline $321 \mathrm{a}$ & $\begin{array}{l}\text { Phylloscopus trochilus } \\
\text { trochilus }\end{array}$ & RB c (?) & $\mathrm{M}, \mathrm{W}$ vc & $\mathrm{Mc}$ & M vc, W irr & $\mathrm{Mc}$ & flu \\
\hline $321 b$ & $\begin{array}{l}\text { Phylloscopus trochilus } \\
\text { acredula }\end{array}$ & - & - & $\mathrm{A}$ & - & - & - \\
\hline 322 & Phylloscopus ibericus & - & - & - & - & $\mathrm{A}$ & - \\
\hline 323 & Phylloscopus collybita & $\mathrm{RB} \mathrm{vc}$ & $\mathrm{M}, \mathrm{W}$ vc, $\mathrm{B}$ ? & $\mathrm{RB}, \mathrm{M}, \mathrm{W} \mathrm{c}$ & RB sc, M, W vc & $\mathrm{RB}, \mathrm{M}, \mathrm{W} \mathrm{c}$ & st \\
\hline 324 & Phylloscopus tristis & - & - & $\mathrm{A}$ & $\mathrm{A}$ & $\mathrm{A}$ & - \\
\hline 325 & Phylloscopus schwarzi & - & - & - & A & A & - \\
\hline 326 & Cettia cetti & $\mathrm{RB} \mathrm{c}$ & RB c & $\mathrm{RB} \mathrm{c}$ & $\mathrm{RB} \mathrm{c}$ & $\mathrm{RB} \mathrm{c}$ & st \\
\hline $327 \mathrm{a}$ & Aegithalos caudatus siculus & $\mathrm{RB} s \mathrm{c}$ & $\mathrm{RB} s \mathrm{~s}$ & $\mathrm{RB} \mathrm{sc}$ & $\mathrm{RB} s \mathrm{~s}$ & $\mathrm{RB} s \mathrm{c}$ & st \\
\hline $327 \mathrm{~b}$ & Aegithalos caudatus italiae & - & - & - & A & A & - \\
\hline 328 & Sylvia atricapilla & $\mathrm{M}, \mathrm{W} \mathrm{vc}, \mathrm{B} \mathrm{sc}$ & $\mathrm{RB}, \mathrm{M} \mathrm{vc}$ & RB c & RB vc, M, W c & RB vc, M, W c & st \\
\hline 329 & Sylvia borin & M vc & M vc & $\mathrm{Mc}$ & $\mathrm{Mc}$ & $\mathrm{Mc}$ & dec \\
\hline 330 & Sylvia deserti & - & - & - & A & A & - \\
\hline 331 & Sylvia nisoria & - & - & A & A & A & - \\
\hline 332 & Sylvia hortensis & M vr & M? & $\mathrm{A}$ & A & M occ & - \\
\hline 333 & Sylvia curruca & M, B vc (?) & M sc & $\mathrm{Mr}$ & $\mathrm{Mr}$ & $\mathrm{M}$ irr & - \\
\hline 334 & Sylvia melanocephala & $\mathrm{RB} v \mathrm{v}$ & RB vc & RB c & $\mathrm{RB}$ vc, $\mathrm{M}$ ? & $\mathrm{RB} \mathrm{vc}$ & st \\
\hline $335 \mathrm{a}$ & Sylvia cantillans cantillans & $\mathrm{Mc}, \mathrm{B} \mathrm{c}$ & $\mathrm{SB} \mathrm{sc}, \mathrm{M} \mathrm{vc}$ & M, SB c & $\mathrm{SB}, \mathrm{M} \mathrm{c}$ & $\mathrm{SB}, \mathrm{Mc}, \mathrm{W}$ occ & st \\
\hline $335 b$ & Sylvia cantillans albistriata & - & - & A & $\mathrm{Mr}$ & $\mathrm{Mr}$ & - \\
\hline 336 & Sylvia subalpina & - & - & - & M sc & M irr? & - \\
\hline 337 & Sylvia ruppeli & - & A & A & A & $\mathrm{M}$ irr & - \\
\hline 338 & Sylvia communis & $\mathrm{M} \mathrm{c}, \mathrm{B} \mathrm{sc}$ & $\mathrm{M} \mathrm{vc}, \mathrm{B} \mathrm{sc}$ & $\mathrm{M}, \mathrm{SB}$ sc & SB sc, M c & SB sc, M c & st \\
\hline 339 & Sylvia conspicillata & $\mathrm{M}, \mathrm{B} \mathrm{sc}$ & $\mathrm{SB}, \mathrm{M} \mathrm{c}$ & $\mathrm{M}, \mathrm{RB}$ sc & $\mathrm{SB}, \mathrm{M} \mathrm{sc}, \mathrm{W}$ r & $\mathrm{SB}, \mathrm{M}, \mathrm{W}$ sc & flu \\
\hline 340 & Sylvia sarda & $\begin{array}{c}\mathrm{M} \mathrm{r}, \mathrm{B} \\
\text { (Pantelleria) }\end{array}$ & $\mathrm{M}, \mathrm{W}$ sc & $\begin{array}{c}\text { M r, RB } \\
\text { (Pantelleria) }\end{array}$ & $\begin{array}{l}\text { M irr, RB } \\
\text { (Pantelleria) }\end{array}$ & $\begin{array}{l}\text { M occ, B Ex } \\
\text { (Pantelleria) }\end{array}$ & dec \\
\hline 341 & Sylvia undata & $\mathrm{RB} \mathrm{r}$ & $\mathrm{M}, \mathrm{W}$ & $\mathrm{RB} \mathrm{sc}, \mathrm{M} \mathrm{r}$ & $\mathrm{RB}, \mathrm{M}, \mathrm{W}$ sc & RB sc, M, W r & in \\
\hline 342 & Sylvia deserticola & - & - & - & - & A & - \\
\hline 343 & Certhia brachydactyla & RB c & $\mathrm{RB} \mathrm{c}$ & RB c & RB c & RB c & st \\
\hline 344 & Sitta europaea & $\mathrm{RB} s \mathrm{c}$ & $\mathrm{RB} \mathrm{sc}$ & $\mathrm{RB} s \mathrm{c}$ & $\mathrm{RB} \mathrm{sc}$ & $\mathrm{RB} s \mathrm{c}$ & st \\
\hline 345 & Tichodroma muraria & A & A & A & A & A & - \\
\hline 346 & Troglodytes troglodytes & $\mathrm{RB} \mathrm{c}$ & $\mathrm{RB} \mathrm{c}, \mathrm{M}$ & RB c & $\mathrm{RB} \mathrm{c}, \mathrm{M} \mathrm{r}$ & $\mathrm{RB} v \mathrm{vc}$ & in \\
\hline 347 & Cinclus cinclus & $\mathrm{RB} s \mathrm{sc}$ & $\mathrm{RB} s \mathrm{sc}$ & $\mathrm{RB} s \mathrm{c}$ & $\mathrm{RB} s \mathrm{~s}$ & $\mathrm{RB} s \mathrm{~s}$ & st \\
\hline 348 & Sturnus vulgaris & $\mathrm{M}, \mathrm{W} \mathrm{vc}$ & $\mathrm{M}, \mathrm{W}$ ve & $\begin{array}{l}\text { M, W vc, B r } \\
\text { (first breed. } \\
\text { 1979) }\end{array}$ & $\mathrm{RB}$ r, M, W vc & $\mathrm{RB} \mathrm{r}, \mathrm{M}, \mathrm{W} \mathrm{c}$ & dec \\
\hline 349 & Sturnus unicolor & $\mathrm{RB} \mathrm{c}$ & $\mathrm{RB} \mathrm{c}$ & $\mathrm{RB} \mathrm{c}$ & $\mathrm{RB} \mathrm{c}$ & $\mathrm{RB} \mathrm{c}$ & st \\
\hline 350 & Pastor roseus & A & M occ & A & A & $\mathrm{M}$ irr & - \\
\hline 351 & Zoothera aurea & - & - & $\mathrm{A}$ & A & A & - \\
\hline 352 & Turdus viscivorus & $\mathrm{M}, \mathrm{W}$ sc, B sc & $\mathrm{M}, \mathrm{W} \mathrm{c}, \mathrm{B} \mathrm{sc}$ & $\mathrm{RB}, \mathrm{M}, \mathrm{W}$ sc & $\mathrm{RB}, \mathrm{M}, \mathrm{W}$ sc & $\mathrm{RB}, \mathrm{M}, \mathrm{W}$ sc & st \\
\hline 353 & Turdus philomelos & $\mathrm{M}, \mathrm{W}$ ve & $\mathrm{M}, \mathrm{W}$ vc & $\mathrm{M}, \mathrm{W} \mathrm{c}$ & $\mathrm{M}, \mathrm{W} \mathrm{c}$ & $\mathrm{M}, \mathrm{W}$ c & dec \\
\hline 354 & Turdus iliacus & $\mathrm{M}, \mathrm{W} \mathrm{r}$ & $\mathrm{M}, \mathrm{W}$ irr & $\mathrm{M}, \mathrm{W} \mathrm{sc}$ & $\mathrm{M}, \mathrm{W}$ sc & $\mathrm{M}, \mathrm{W}$ irr, r & flu \\
\hline 355 & Turdus merula & $\mathrm{M}, \mathrm{W}$ vc, B sc & RB sc, M, W & $\mathrm{M}, \mathrm{W}, \mathrm{RB} \mathrm{c}$ & $\mathrm{RB}, \mathrm{M}, \mathrm{W} \mathrm{c}$ & $\mathrm{RB}, \mathrm{M}, \mathrm{W} \mathrm{c}$ & in \\
\hline 356 & Turdus pilaris & $\mathrm{M}, \mathrm{W} \mathrm{sc}$ & $\mathrm{M}, \mathrm{W}$ irr & $\mathrm{M}, \mathrm{W}$ sc & $\mathrm{M}, \mathrm{W} \mathrm{sc}$ & M sc, W irr & flu \\
\hline $357 \mathrm{a}$ & Turdus torquatus torquatus & M sc & M, W irr & $\mathrm{M}, \mathrm{W} \mathrm{sc}$ & M sc, W irr & $\mathrm{M}, \mathrm{W}$ irr & - \\
\hline
\end{tabular}




\begin{tabular}{|c|c|c|c|c|c|c|c|}
\hline $357 \mathrm{~b}$ & Turdus torquatus alpestris & M sc & $\mathrm{M}, \mathrm{W}$ irr & $\mathrm{M}, \mathrm{W} \mathrm{sc}$ & M sc, W irr & $\mathrm{M}, \mathrm{W}$ irr & - \\
\hline 358 & Turdus atrogularis & - & - & - & - & A & - \\
\hline 359 & Cercotrichas galactotes & A & A & A & $M$ irr & M irr & - \\
\hline $360 \mathrm{a}$ & Muscicapa striata striata & SB sc, M c & SB sc, $M$ vc & $\mathrm{SB}, \mathrm{M} \mathrm{sc}$ & SB sc, M c & SB sc, M c & flu \\
\hline $360 \mathrm{~b}$ & Muscicapa striata tyrrhenica & - & - & - & M? & $\mathrm{Mr}, \mathrm{B}$ occ & - \\
\hline 361 & Erithacus rubecula & $\mathrm{M}, \mathrm{W}$ vc, B sc & $\mathrm{M}, \mathrm{W}$ vc, B sc & $\mathrm{M}, \mathrm{W}$ c, RB sc & RB sc, M, W vc I & $\mathrm{RB} \mathrm{sc}, \mathrm{M}, \mathrm{W} \mathrm{vc}$ & st \\
\hline $362 \mathrm{a}$ & Cyanecula svecica svecica & - & - & M irr & $\mathrm{M}$ irr & M irr & - \\
\hline $362 b$ & Cyanecula svecica cyanecula & $\mathrm{M}, \mathrm{W} \mathrm{sc}$ & M irr, W sc & $\mathrm{M}, \mathrm{W} \mathrm{sc}$ & $\mathrm{M} \mathrm{sc}, \mathrm{W} \mathrm{r}$ & $\mathrm{M}, \mathrm{W} \mathrm{r}$ & dec \\
\hline 363 & Luscinia luscinia & - & - & - & A & A & - \\
\hline 364 & Luscinia megarhynchos & $\mathrm{M}, \mathrm{B} \mathrm{c}$ & SB sc, M vc & M, SB c & $\mathrm{SB}, \mathrm{M} \mathrm{c}$ & $\mathrm{SB}, \mathrm{M} \mathrm{sc}$ & dec \\
\hline 365 & Tarsiger cyanurus & - & - & - & A & A & - \\
\hline 366 & Ficedula parva & - & - & $\mathrm{Mr}$ & $\mathrm{M}$ irr & $\mathrm{Mr}$ & - \\
\hline 367 & Ficedula semitorquata & - & - & A & A & A & - \\
\hline 368 & Ficedula hypoleuca & M sc & M sc & M sc & $\mathrm{Mc}$ & $\mathrm{Mc}$ & in \\
\hline 369 & Ficedula albicollis & $\mathrm{Mc}$ & $\mathrm{M} \mathrm{c}$ & M sc & M sc, B occ & M sc, B occ & dec \\
\hline 370 & Phoenicurus ochruros & $\mathrm{RB} \mathrm{c}$ & $\mathrm{RB}, \mathrm{M}, \mathrm{W} \mathrm{c}$ & $\mathrm{RB}, \mathrm{M}, \mathrm{W} \mathrm{c}$ & $\mathrm{RB}, \mathrm{M}, \mathrm{W} \mathrm{c}$ & $\mathrm{RB}, \mathrm{M}, \mathrm{W} \mathrm{c}$ & st \\
\hline 371 & Phoenicurus phoenicurus & $\mathrm{M} \mathrm{c}, \mathrm{SB} \mathrm{r}$ & $\mathrm{M} \mathrm{vc}, \mathrm{B}$ ? W? & $\mathrm{M} \mathrm{c}, \mathrm{SB}$ vr & M c, B occ & $\mathrm{M} \mathrm{c}, \mathrm{B}$ occ & st \\
\hline 372 & Phoenicurus moussieri & - & - & A & $\mathrm{A}$ & $\begin{array}{c}\text { M irr (Pantel- } \\
\text { leria and } \\
\text { Lampedusa) }\end{array}$ & - \\
\hline 373 & Monticola saxatilis & SB r, M c & $\mathrm{SB}, \mathrm{M} \mathrm{sc}$ & SB r, M sc & $\mathrm{SB}, \mathrm{M} \mathrm{sc}$ & SB r, M sc & flu \\
\hline 374 & Monticola solitarius & $\mathrm{RB} \mathrm{vc}$ & $\mathrm{RB} \mathrm{c}$ & $\mathrm{RB} \mathrm{c}$ & RB c, M irr & $\mathrm{RB}$ c, $\mathrm{M}, \mathrm{W} \mathrm{r}$ & dec \\
\hline 375 & Saxicola rubetra & $\mathrm{B} \mathrm{r}, \mathrm{M} \mathrm{c}$ & $\mathrm{B}, \mathrm{Mc}$ & $\mathrm{Mc}$ & $\mathrm{Mc}, \mathrm{W}$ occ & $\mathrm{Mc}$ & st \\
\hline 376 & Saxicola torquatus rubicola & RB sc, M, W vc & $\mathrm{RB}, \mathrm{M}, \mathrm{W}$ & $\mathrm{RB}, \mathrm{M}, \mathrm{W} \mathrm{c}$ & $\mathrm{RB}, \mathrm{M}, \mathrm{W} \mathrm{c}$ & $\mathrm{RB}, \mathrm{M}, \mathrm{W} \mathrm{c}$ & in \\
\hline $377 \mathrm{a}$ & Oenanthe oenanthe oenanthe & SB r, M vc & $\mathrm{M} \mathrm{vc}, \mathrm{B} \mathrm{sc}$ & $\mathrm{Mc}, \mathrm{SB} \mathrm{sc}$ & $\mathrm{SB}, \mathrm{M} \mathrm{c}$ & $\mathrm{SB}, \mathrm{M} \mathrm{c}$ & in \\
\hline $377 \mathrm{~b}$ & Oenanthe oenanthe leucorhoa & - & - & - & $\mathrm{A}$ & M vr & - \\
\hline 378 & Oenanthe isabellina & - & - & $\mathrm{Mr}$ & $\mathrm{Mr}$ & $\mathrm{Mr}$ & - \\
\hline 379 & Oenanthe deserti & - & - & A & A & M irr & - \\
\hline $380 \mathrm{a}$ & Oenanthe hispanica hispanica & M sc & M sc & M sc & M sc & M sc & st \\
\hline $380 \mathrm{~b}$ & $\begin{array}{l}\text { Oenanthe hispanica } \\
\text { melanoleuca }\end{array}$ & M sc & M sc & $\mathrm{M} \mathrm{sc}, \mathrm{SB}$ r & $\mathrm{M} \mathrm{sc}, \mathrm{B} \mathrm{r}$ & M sc, B occ, & st \\
\hline 381 & Oenanthe pleschanka & - & - & - & A & A & - \\
\hline 382 & Oenanthe leucura & M? & A & $\mathrm{M}$ irr, vr & A & A & - \\
\hline 383 & Oenanthe leucopyga & - & - & - & - & $\mathrm{A}$ & - \\
\hline 384 & Regulus regulus & $\mathrm{M}, \mathrm{W}, \mathrm{B} \mathrm{sc}$ & $\mathrm{M}, \mathrm{W} \mathrm{sc}, \mathrm{B} ?$ & $\mathrm{M}, \mathrm{W} \mathrm{sc}$ & $\mathrm{M}, \mathrm{W}$ sc & $\mathrm{M}, \mathrm{W}$ sc & st \\
\hline 385 & Regulus ignicapilla & $\mathrm{RB} \mathrm{c}, \mathrm{M}, \mathrm{W} \mathrm{c}$ & $\mathrm{RB} \mathrm{c}, \mathrm{M}, \mathrm{W} \mathrm{vc}$ & $\mathrm{RB}, \mathrm{M}, \mathrm{W}$ sc & $\mathrm{RB}, \mathrm{M}, \mathrm{W}$ sc $\mathrm{I}$ & $\mathrm{RB} \mathrm{c}, \mathrm{M}, \mathrm{W} \mathrm{sc}$ & flu \\
\hline 386 & Bombycilla garrulus & - & - & A & A & A & - \\
\hline 387 & Prunella collaris & $\mathrm{M}, \mathrm{W} \mathrm{r}$ & $\mathrm{M}, \mathrm{W} \mathrm{r}$ & M, W sc & $\mathrm{M}, \mathrm{W} \mathrm{sc}$ & $\mathrm{M}, \mathrm{W} \mathrm{r}$ & st \\
\hline 388 & Prunella modularis & $\mathrm{M}, \mathrm{W}$ vc & $\mathrm{M}, \mathrm{W}$ c & $\mathrm{M}, \mathrm{W}$ sc & $\mathrm{M}, \mathrm{W}$ sc & $\mathrm{M}, \mathrm{W}$ sc & vdec \\
\hline 389 & Passer domesticus & - & - & - & A & A & - \\
\hline 390 & Passer italiae & B? & B? (Messina) & RB? (Aeolian Is.) & RB? & RB sc & st \\
\hline 391 & Passer hispaniolensis & $\mathrm{RB} \mathrm{c}$ & $\mathrm{RB} \mathrm{vc}$ & $\mathrm{RB} v \mathrm{c}$ & RB vc, M, W sc I & $\mathrm{RB} \mathrm{c}, \mathrm{M}, \mathrm{W} \mathrm{sc}$ & flu \\
\hline 392 & Passer montanus & RB sc & $\mathrm{RB}, \mathrm{M}$ & RB sc & $\mathrm{RB}, \mathrm{M} \mathrm{sc}$ & $\mathrm{RB} \mathrm{c}$ & flu \\
\hline 393 & Petronia petronia & $\mathrm{RB} \mathrm{sc}$ & $\mathrm{RB} \mathrm{c}$ & $\mathrm{RB} s \mathrm{~s}$ & $\mathrm{RB} s \mathrm{~s}$ & $\mathrm{RB}, \mathrm{M}, \mathrm{W}, \mathrm{sc}$ & st \\
\hline 394 & Montifringilla nivalis & - & - & $\mathrm{A}$ & $\mathrm{A}$ & $\mathrm{A}$ & - \\
\hline 395 & Anthus trivialis & $\mathrm{M} \mathrm{vc}$ & $\mathrm{M} \mathrm{vc}$ & M c, B occ & M c, W, B irr & $\mathrm{Mc}$ & dec \\
\hline 396 & Anthus hodgsoni & - & - & - & - & M irr, W occ & - \\
\hline 397 & Anthus cervinus & M irr & M sc & M sc & M sc, W irr & M r, W irr & flu \\
\hline
\end{tabular}




\begin{tabular}{|c|c|c|c|c|c|c|c|}
\hline 398 & Anthus pratensis & $\mathrm{M}, \mathrm{W}$ vc & $\mathrm{M}, \mathrm{W}$ vc & $\mathrm{M}, \mathrm{W} \mathrm{c}$ & $\mathrm{M}, \mathrm{W} \mathrm{c}$ & $\mathrm{M}, \mathrm{W} \mathrm{c}$ & dec \\
\hline 399 & Anthus spinoletta & M irr, $r$ & M sc & $\mathrm{M}, \mathrm{W}$ sc & $\mathrm{M}, \mathrm{W}$ sc & $\mathrm{M}, \mathrm{W} \mathrm{r}$ & flu \\
\hline 400 & Anthus petrosus & - & - & - & A & A & - \\
\hline 401 & Anthus richardi & $\mathrm{Mr}$ & $\mathrm{Mr}$ & $\mathrm{Mr}$ & $\mathrm{M}, \mathrm{W} \mathrm{r}$ & $\mathrm{M}, \mathrm{W} \mathrm{r}$ & flu \\
\hline 402 & Anthus godlewskii & - & - & - & - & A & - \\
\hline 403 & Anthus campestris & $\mathrm{M} \mathrm{c}, \mathrm{B} \mathrm{sc}$ & M sc & $\mathrm{M} \mathrm{sc}, \mathrm{SB}$ r & SB sc, Msc, W irr & SB r, M sc & dec \\
\hline $404 \mathrm{a}$ & Motacilla flava flava & $\mathrm{Mc}$ & $\mathrm{M} \mathrm{vc}$ & $\mathrm{Mc}$ & $\mathrm{Mc}$ & $\mathrm{Mc}$ & - \\
\hline $404 \mathrm{~b}$ & Motacilla flava feldegg & $\mathrm{Mc}$ & $\mathrm{Mc}$ & M c, B occ & Mc, Wr, B occ & $\mathrm{Mc}, \mathrm{Br}, \mathrm{W}$ occ & st \\
\hline $404 \mathrm{c}$ & Motacilla flava cinereocapilla & $\mathrm{Mc}$ & - & $\mathrm{Mc}$ & $\mathrm{Mc}$ & $\mathrm{Mc}$ & - \\
\hline $404 d$ & Motacilla flava beema & - & - & - & $\mathrm{Mr}$ & $\mathrm{Mr}$ & - \\
\hline $404 \mathrm{e}$ & Motacilla flava flavissima & - & - & A & A & A & - \\
\hline $404 \mathrm{f}$ & Motacilla flava iberiae & - & - & A & A & A & - \\
\hline $404 \mathrm{~g}$ & Motacilla flava thunbergi & M sc & M sc & M sc & M sc & M sc & - \\
\hline 405 & Motacilla cinerea & $\mathrm{RB} \mathrm{c}, \mathrm{M} \mathrm{c}$ & $\mathrm{RB}, \mathrm{M} \mathrm{sc}$ & $\mathrm{RB}, \mathrm{M}, \mathrm{W}$ sc & $\mathrm{RB}, \mathrm{M}, \mathrm{W}$ sc & $\mathrm{RB} \mathrm{c}, \mathrm{M}, \mathrm{W} \mathrm{sc}$ & st \\
\hline 406 & Motacilla citreola & - & A? & $\mathrm{A}$ & A & M irr, W occ & - \\
\hline $407 \mathrm{a}$ & Motacilla alba alba & $\mathrm{M}, \mathrm{W}$ vc, B r & $\mathrm{M}, \mathrm{W}$ vc & RB sc, M, W c & RB sc, M, W c & RB sc, M, W c & dec \\
\hline $407 \mathrm{~b}$ & Motacilla alba yarrelli & - & A & A & A & A & - \\
\hline $408 \mathrm{a}$ & Fringilla coelebs coelebs & M, W vc, B r & M, W vc, B sc & $\mathrm{RB}, \mathrm{M}, \mathrm{W} \mathrm{c}$ & $\mathrm{RB}, \mathrm{M}, \mathrm{W} \mathrm{c}$ & RB, M, W sc & flu \\
\hline $408 b$ & Fringilla coelebs africana & - & - & $\begin{array}{c}\mathrm{A} \\
\text { (Pantelleria) }\end{array}$ & $\begin{array}{c}\text { A, B occ } \\
\text { (Lampedusa) }\end{array}$ & $\begin{array}{c}\text { A, B occ } \\
\text { (Lampedusa) }\end{array}$ & - \\
\hline 409 & Fringilla montifringilla & $\mathrm{M}, \mathrm{W} \mathrm{r}$ & $\mathrm{M}, \mathrm{W} \mathrm{r}$ & $\mathrm{M}, \mathrm{W}, \mathrm{r}$ & $\mathrm{M}, \mathrm{W} \mathrm{r}$ & M, W irr & st \\
\hline 410 & Coccothraustes coccothraustes & $\mathrm{M}, \mathrm{W} \mathrm{c}$ & $\mathrm{M}, \mathrm{W}$ vc & $\mathrm{M}, \mathrm{W}$ sc & $\mathrm{M}, \mathrm{W} \mathrm{sc}$ & $\mathrm{M}, \mathrm{W} \mathrm{r}$ & vdec \\
\hline 411 & Carpodacus erythrinus & A & - & - & A & A & - \\
\hline 412 & Pyrrhula pyrrhula & A & M, W occ & A & A & A & - \\
\hline 413 & Bucanetes githagineus & A & A & M irr, $r$ & A & M irr & - \\
\hline 414 & Chloris chloris & $\mathrm{M}, \mathrm{W}$ vc, B, r & $\mathrm{M}, \mathrm{W}, \mathrm{B} \mathrm{sc}$ & RB sc, M, W c & $\mathrm{RB} \mathrm{sc}, \mathrm{M}, \mathrm{W} \mathrm{c}$ & $\mathrm{RB} \mathrm{c}, \mathrm{M}, \mathrm{W} \mathrm{sc}$ & in \\
\hline 415 & Linaria flavirostris & - & - & - & A & A & - \\
\hline 416 & Linaria cannabina & M, W vc, B sc & $\mathrm{M}, \mathrm{W}$ vc, B r & $\mathrm{RB}, \mathrm{M}, \mathrm{W} \mathrm{c}$ & RB, M, W c & RB, M, W c & in \\
\hline 417 & Acanthis flammea cabaret & - & A & A & A & A & - \\
\hline 418 & Loxia curvirostra & M irr, sc & M irr, sc & RB r, M, W irr & RB sc, M, W irr & RB r, M, W irr & flu \\
\hline 419 & Carduelis carduelis & $\mathrm{M}, \mathrm{W}$ vc, B c & $\mathrm{RB} v \mathrm{v}$ & $\mathrm{RB}, \mathrm{M}, \mathrm{W} \mathrm{c}$ & RB vc, M W c & $\mathrm{RB}, \mathrm{M}, \mathrm{W} \mathrm{c}$ & flu \\
\hline 420 & Carduelis citrinella & - & - & - & A & A & - \\
\hline 421 & Serinus serinus & M, W vc, B sc & $\mathrm{M}, \mathrm{W}, \mathrm{RB}$ vc & $\mathrm{RB}, \mathrm{M}, \mathrm{W} \mathrm{c}$ & RB vc, M, W c & $\mathrm{RB}, \mathrm{M}, \mathrm{W} \mathrm{c}$ & flu \\
\hline 422 & Spinus spinus & $\mathrm{M}, \mathrm{W}$ irr, r & $\mathrm{M}, \mathrm{W}$ irr, r & $\mathrm{M}, \mathrm{W}$ r, RB vr & RB r, N, W sc & RB, M, W sc & st \\
\hline 423 & Calcarius lapponicus & - & - & - & A & A & - \\
\hline 424 & Plectrophenax nivalis & - & - & $\mathrm{M}$ irr, $\mathrm{r}$ & M occ & M occ & - \\
\hline 425 & Emberiza melanocephala & A & A & M irr, $r$ & M occ & M occ & - \\
\hline 426 & Emberiza bruniceps & - & - & A & A & A & - \\
\hline 427 & Emberiza calandra & $\mathrm{RB} \mathrm{c}$ & $\mathrm{RB}, \mathrm{M} \mathrm{vc}$ & $\mathrm{RB} \mathrm{c}, \mathrm{M}, \mathrm{W} \mathrm{sc}$ & $\mathrm{RB} \mathrm{c}, \mathrm{M}, \mathrm{W} \mathrm{sc}$ & $\mathrm{RB}, \mathrm{M}, \mathrm{W} \mathrm{c}$ & flu \\
\hline 428 & Emberiza cia & RB sc & $\mathrm{RB} \mathrm{c}$ & RB sc & $\mathrm{RB} s \mathrm{~s}$ & $\mathrm{RB} \mathrm{sc}$ & st \\
\hline 429 & Emberiza hortulana & $\mathrm{M}, \mathrm{B}(?), \mathrm{W} \mathrm{c}$ & M, B (?), W sc & M irr, sc & M irr, vr & M irr, vr & vdec \\
\hline 430 & Emberiza caesia & A & - & - & - & A & - \\
\hline 431 & Emberiza cirlus & $\mathrm{RB} \mathrm{c}$ & $\mathrm{RB} \mathrm{c}$ & $\mathrm{RB} \mathrm{c}$ & RB c, M, W sc & $\mathrm{RB}, \mathrm{M}, \mathrm{W} \mathrm{c}$ & st \\
\hline 432 & Emberiza citrinella & $\mathrm{Mr}$ & $\mathrm{M}, \mathrm{W}$ sc & A & A & A & - \\
\hline 433 & Emberiza schoeniclus & $\mathrm{M}, \mathrm{W} \mathrm{c}, \mathrm{B} \mathrm{r}$ & $\mathrm{M}, \mathrm{W} \mathrm{c}$ & M, W sc & M, W sc & $\mathrm{M}, \mathrm{W} \mathrm{r}$ & dec \\
\hline 434 & Emberiza pallasi & - & - & - & - & A & - \\
\hline 435 & Emberiza aureola & - & - & - & A & A & - \\
\hline 436 & Emberiza rustica & - & - & A & A & A & - \\
\hline 437 & Emberiza pusilla & A & - & A & A & $\mathrm{Mr}$ & - \\
\hline
\end{tabular}




\begin{tabular}{|l|l|l|}
\hline 1. Colinus virginianus & 11. Anas erythrorhyncha & 21. Agapornis roseicollis \\
\hline 2. Alectoris chukar & 12. Streptopelia roseogrisea & 22. Agapornis fischeri \\
\hline 3. Alectoris rufa & 13. Geronticus eremita & 23. Corvus albus \\
\hline 4. Chrysolophus pictus & 14. Threskiornis aethiopicus & 24. Leiothrix lutea \\
\hline 5. Cygnus atratus & 15. Haliaeetus vocifer & 25. Gracula religiosa \\
\hline 6. Alopochen aegyptiaca & 16. Nymphicus hollandicus & 26. Lamprotornis superbus \\
\hline 7. Bucephala albeola & 17. Psittacus erithacus & 27. Passer luteus \\
\hline 8. Tadorna cana & 18. Thectocercus acuticaudatus & 28. Ploceus subaureus \\
\hline 9. Aix galericulata & 19. Psittacula eupatria & 29. Amandava amandava \\
\hline 10. Cairina moschata & 20. Melopsittacus undulatus & \\
\hline
\end{tabular}

Table 2. List of the alien species presently known in Sicily. Nomenclature according to Gill et al. (2020).

\section{DISCUSSION}

\section{Some comments on the check-list}

Some general comments on the trend will be discussed in the next section; we report here some annotations on the former checklists. The Middle Spotted Woodpecker Leiopicus medius is reported by Doderlein who in turn mentions it as it was recorded on the Madonie Mts. by Minà Palumbo (1857); however, Minà Palumbo had depicted what he thought was a Middle Spotted Woodpecker, which actually turned out to be a young Great Spotted Woodpecker (Massa \& Sarà, 2011). Further, Doderlein mentions also the Pin-tailed Sandgrouse Pterocles alchata, as having been precisely depicted by Cupani (1713), but neither Riggio (1892) nor Priolo (1996) listed this species among the Cupani's birds of Panphyton Siculum, and really this species is not reported in the Cupani's (1713) book. Iapichino \& Massa (1989) omitted this species from Sicilian birds; however, Giglioli (1907) recorded one adult specimen captured at Siracusa in 1859 (see also Corso, 2005). Thus, this species may be considered as a former accidental in Sicily. Concerning the breeding of the Lesser Black-backed Gull Larus fuscus, recorded by Doderlein (1869-1874), who described the characters of one young reared by him, they match more those of the Yellow-legged Gull Larus michahellis than the previous species, and this is more consistent with the breeding distribution of the two species. Whitaker (1920) reports the Brent Goose Branta bernicla as 'winter migrant, at times not uncommon'; it seems unlikely because this species has been always considered rather rare in Italy (Ar- rigoni degli Oddi, 1929), it has not been recorded by Doderlein before Whitaker and has been considered as accidental by the following authors after Whitaker (e.g., Iapichino \& Massa, 1989; Corso, 2005), and in the Whitaker collection there are no specimens of this bird (Lo Valvo \& Massa, 2000). Also the Black Vulture Aegypius monachus, recorded by Doderlein on Benoit's authority and by Whitaker, probably on Doderlein's authority, actually was not reported by Benoit (1840) (see Iapichino \& Massa, 1989).

The breeding of the Demoiselle Crane Anthropoides virgo on Lampedusa Is. has not been reported by Doderlein (1869-1874), who considered it as an accidental bird, but it was hypothesized by La Mantia (2001) on the basis of some historical data; further, Massa et al. (2015) have highlighted that because certainly a Crane species bred on the island in the first half of 1800, on the basis of the environmental and geographical characteristics of the island, it could not be other than the Demoiselle Crane.

Arrigoni degli Oddi (1929) and Iapichino \& Massa (1989) considered unreliable the capture of the Black-crowned Crane Balearica pavonina on the Lampedusa Is. (specimen preserved in the $\mathrm{Mu}-$ seum of Zoology of Palermo's University, cited by Swainson, 1936), but in the light of another specimen collected on Lampedusa before 1870 and preserved in the Museum of Zoology of the Pavia's University, Massa et al. (2015) have considered possible the former rare presence of this species in Sicily. About the specimen of Museum of Zoology of Palermo, Orlando (1935) highlighted that it was part of the old small collection extant before Doderlein arrived in Palermo (1862), and Doderlein himself had labeled the specimen 'Lampedusa'. 
Thus, we now consider reliable the old presence of this species in the Lampedusa Is.

Neither Doderlein (1869-1874) nor Whitaker (1920) cite the Pallid Swift Apus pallidus, but they, as well as Minà Palumbo (1857) very probably identified also late summer-autumnal individuals as Common Swift Apus apus, because the Pallid Swift was little known or unknown (it was described in 1870). Nevertheless, in the Whitaker collection there are not Italian Pallid Swifts (Lo Valvo \& Massa, 2000), even if this species was known in Sicily before its formal description, having Cupani (1713) depicted clearly it in the Panphyton Siculum (Priolo, 1996); however, that drawing was considered to belong to a Common Swift by Riggio (1892), who was contemporaneous of Doderlein. Thus, it seems obvious that the Pallid Swift was regularly present in Sicily also in the half of 1800 .

In addition, the following species listed by Whitaker (1920) have never been found as breeding in Sicily: Winchat Saxicola rubetra, Aquatic Warbler Acrocephalus paludicola, Lesser Whitethroat Sylvia curruca, Wood Warbler Phylloscopus sibilatrix, Ortolan Bunting Emberiza hortulana, Lesser Spotted Eagle Clanga pomarina, Northern Goshawk Accipiter gentilis, Corn Crake Crex crex, Lesser Black-baked Gull Larus fuscus. Concerning Winchat, Lesser Whitethroat, Wood Warbler and Ortolan Bunting, they are very probably erroneously reported as breeding by Doderlein (18691874); the Lesser Spotted Eagle was erroneously reported as sedentary in Sicily by Doderlein (18691874) certainly on the Benoit (1840) authority. Interestingly, not the Lesser Spotted Eagle, but the Greater Spotted Eagle Clanga clanga was figured by Cupani (1713) in the Panphyton Siculum (Priolo, 1996); it was misidentified as Golden Eagle Aquila chrysaetos by Benoit (1840) and Riggio (1892). Most likely the Marsh Harrier was really a breeding bird in the wide marshland of 1800s, while nowadays it finds hardly suitable breeding habitats.

Further, the Common Redstart Phoenicurus phoenicurus has never been found as wintering in Sicily; Iapichino \& Massa (1989) established that all Carrion Crows Corvus corone corone recorded from Sicily actually belong to young Rooks Corvus frugilegus. Additionally, the Lesser Kestrel Falco naumanni is known to be a summer migrant and breeding species in Sicily, wintering in tropical Africa, but Whitaker (1920) reports it as resident; no specimens collected in winter are present in the Whitaker collection (Lo Valvo \& Massa, 2000) and very few winter records are known from the island (Iapichino \& Massa, 1989; Corso, 2005). Thus, the status recorded by Whitaker is very probably a mistake.

The Pheasant Phasianus colchicus was introduced in 1800 and became extinct before the half of the same century (Doderlein, 1869-1874); in 1980s it was again introduced into a game reserve in the province of Enna, but there are not proofs of breeding in the wild. The Red-crested Pochard Netta rufina, formerly breeding in Sicily, presently is only migrant and occasionally wintering. The Great-crested Grebe Podiceps cristatus and the Black-necked Grebe Podiceps nigricollis were considered extinct as breeding birds in 1958-1960 and 1965, respectively. The Little Crake Zapornia parva bred occasionally in 1980 .

The presence of the Nutcracker Nucifraga caryocatactes reported by Doderlein and Whitaker from Sicily seems very unlikely, but we list it because a specimen from Malta is present in the Whitaker collection (Lo Valvo \& Massa, 2000).

Finally, the presence in Sicily of the Northern Bald Ibis Geronticus eremita (alien species) is due to the arrival of four or five individuals in October 2017 first at Alicudi and Salina (Aeolian Islands), then at Palazzolo Acreide (Siracusa) and inside the racecourse of Palermo. They escaped from the Fagagna center (Udine, north Italy) and later some of them were captured and brought back to the center.

\section{Considerations on the trend over the long- term period considered}

To date, no one has discovered another geographical area like the Mediterranean anywhere else in the world, in Europe, Asia or Africa; this sentence dates back to 1598 and is attributed to the Jesuit and Spanish writer José de Acosta. Even if more than four hundred years have passed, this sentence is still valid, the Mediterranean has unique peculiarities not only for its almost closed sea, but for its emerged lands, the three peninsulas and the numerous islands, including Sicily, Sardinia, Corsica, Balearics, Cyprus and Crete, which represent jewels from the faunistic and floristic points of view. However, this wonderful land has received a bad attention from the people inhabiting it, who has degraded a very large surface. The islands have particularly 
suffered from the degrading action by man. This depended also on the population growth and the consequent pressure on natural environments.

We have to make two preliminary remarks: i) one hundred years ago in Sicily there were very few roads and railways, transports were very difficult if not impossible in some inland areas; ii) the habitat of many plains was very rich in marshes and humid zones inhabited by thousands of waterbirds (Doderlein, 1869-1874). Thus, the ornithological exploration of the island was limited and possibly some inland species were overlooked. The marshland reclamation, already started in the first years of 1900 (Whitaker, 1920), was the most important reason of the extinction or declining of waterbirds. $\mathrm{Cu}$ pani (1713) depicted the Smew Mergellus albellus in the Panphyton Siculum, Doderlein (1869-1874) reported it as regularly wintering in Sicily, Whitaker (1920) only occasionally wintering and subsequent authors as 'accidental'; this status fits for other ducks and waterbirds and is likely dependent on the marshland surface drastic reduction in the island.

In the last one hundred of years Sicily changed very much, due to the population growth (presently ca. 5 millions in $25,711 \mathrm{~km}^{2}$ ), road construction, habitat change, etc. The availability of carcasses for necrophagous birds was declining and species like the Lammergeier Gypaetus barbatus and Griffon Vulture Gyps fulvus, already known and depicted at the time of Cupani (1713), decreased dramatically and became extinct. In simple terms, most species declines may be explained by the anthropization and environment trivialization of the island.

Some waders changed very much their habits and their wintering in Sicily has been noticed more and more frequently. The Wood Sandpiper Tringa glareola, together with other waders like Pied Avocet Recurvirostra avosetta, Black-winged Stilt $\mathrm{Hi}$ mantopus himantopus, Black-tailed Godwit Limosa limosa, Bar-tailed Godwit Limosa lapponica, Turnstone Arenaria interpres, Temminck's Stint Calidris temminckii, Spotted Redshank Tringa erythropus, Greenshank Tringa nebularia and Marsh Sandpiper Tringa stagnatilis begun to winter more and more regularly than formerly, when no author reported them as wintering (Surdo, 2016; Surdo et al., 2018; Surdo \& Biondi, 2019). This may depend on a greater coverage of ornithological exploration, the use of increasingly professional tools (telescopes, cameras with powerful telephoto lenses) and greater knowledge and ability to recognize these species, but probably also on the climate change.

We discuss below the trend categories separately.

As reported above, 437 species are listed in the Table 1; 155 of them are irregular or accidental and are not considered in the next discussion; besides, in the final discussion the subspecies were not considered as separate taxa. Out of 283 species considered in the Table 3, 75 (26.5\%) resulted to maintain stable populations through the long period here considered of one hundred and fifty years, $11(3.9 \%)$ became extinct, 35 (12.4\%) resulted increasing, 12 $(4.2 \%)$ very increasing, $75(26.5 \%)$ with declining populations and $34(12 \%)$ with very declining populations. Further, 41 species (14.5\%) showed a population trend different from the previous ones, that we classified as fluctuating (Fig. 1).

\begin{tabular}{|l|l|}
\hline Species with stable population & 75 \\
\hline Species with increasing population & 35 \\
\hline Species with very increasing populations & 12 \\
\hline Species with declining populations & 75 \\
\hline Species with very declining populations & 34 \\
\hline Species with fluctuating population & 41 \\
\hline Species became extinct & 11 \\
\hline
\end{tabular}

Table 3. Number of breeding and migrant Sicilian bird species $(\mathrm{n}=282)$, whose populations showed a different trend over one hundred and fifty years.

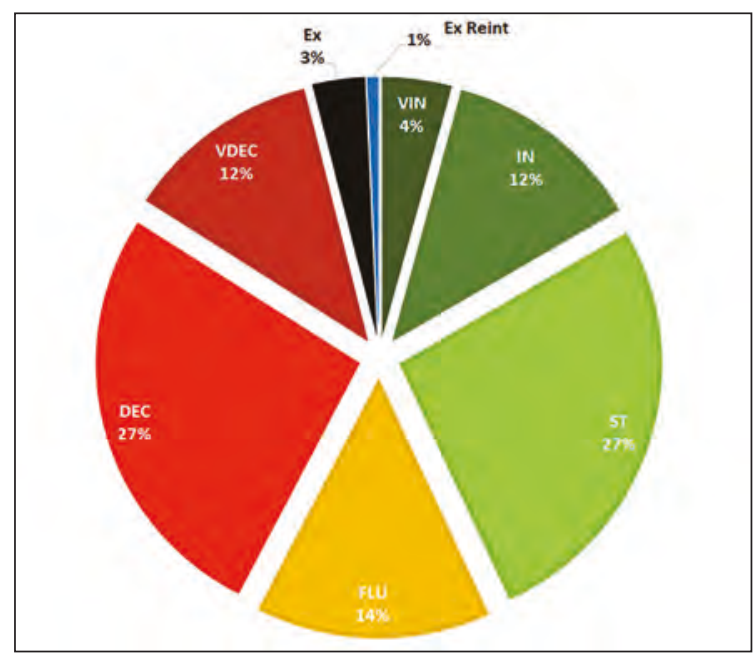

Figure 1. Percentage of breeding and migrant birds, whose populations showed a different trend over one hundred and fifty years. Ex = extinct; Ext Reint = Extinct reintroduced; in = increasing; vin = very increasing; $\mathrm{dec}=$ declining; $\mathrm{vdec}$ = very declining; $\mathrm{st}=$ stable; flu = fluctuating 
We discuss below on some representatives of these categories, mixing together the declining with the very declining species and the increasing with the very increasing species.

\section{i) Stable species (st)}

Generally species with stable populations have a wide ecological niche and may adapt easily to environment changes. The list of stable species in Sicily is rather long and amounts to 75 . We discuss here of only thirteen species, that may be considered good representatives of the category: Common Moorhen Gallinula chloropus, Grey Heron Ardea cinerea, Eurasian Stone Curlew Burhinus oedicnemus, Northern Long-eared Owl Asio otus, Tawny Owl Strix aluco, Short-toed Snake-eagle Circaetus gallicus, Great Tit Parus major, Blue Tit Cyanistes caeruleus, Woodlark Lullula arborea, Zitting Cisticola Cisticola juncidis, Sicilian Long-tailed Tit Aegithalos caudatus siculus, Eurasian Nuthatch Sitta europaea, European Robin Erithacus rubecula.

Common Moorhen Gallinula chloropus. The Moorhen is the most widespread rallid, also present in very small ponds, rivulets and even within fishponds in town gardens, like the Botanical Garden of Palermo. Their adaptation abilities are certainly the reasons of the huge numbers present in the island.

Grey Heron Ardea cinerea. While the Grey Heron maintained stable populations over the period here considered, the Purple Heron Ardea purpurea has decreased, and very probably the latter once was at least as common as the former. This seems an opposing trend compared to other Italian regions (Fasola et al., 2010), but probably it depends on the scarce presence of suitable habitats for breeding by Purple Heron in the island.

Eurasian Stone Curlew Burhinus oedicnemus (Fig. 2). It is one of the most elusive birds in Europe; monitoring of this bird is very difficult, even if it frequently emits a very characteristic and unmistakable sound. In the last fifteen years many new breeding pairs have been found and it possibly is recently increasing. Pairs of this bird may be found breeding also on the edges of airports and dry plains far from rivers and humid zones. This might depend on its wide ecological niche, also regarding the feeding habits; as it has been observed in many instances, indeed it may prey upon small vertebrates, mammals, other birds, reptiles, amphibians, and arthropods.
Stone Curlews slightly avoid agricultural activities, towards which they show only a little tolerance; in addition they are extremely intolerant to disturbances related to fires (Triolo et al., 2011). The Sicilian breeding population has the colors of the feathers somewhere between the ssp. oedicnemus and the ssp. saharae, probably the result of a clinal variation.

Northern Long-eared Owl Asio otus and Tawny Owl Strix aluco. Long-eared and Tawny Owls resulted to have in Sicily stable populations. While the first species does not vocalize much, the second is rather easy to census through the sound that it emits in the night. Thus, the presence of the Longeared Owl may be overlooked.

Short-toed Snake-eagle Circaetus gallicus. It seems that the Short-toed Snake-eagle has a resident small population in Sicily; in spite of its large size, its breeding has never been confirmed, while its winter and summer presence has been recorded since 1980s. Recently it has been proposed that Italian populations of this raptor follow a circuitous autumnal migration from Italy to Iberian peninsula and to Africa (Agostini et al., 2016). However, this cannot depend on its unfitness to cover large water barriers, because the Short-toed Snake-eagle was regularly observed by Doderlein (1869-1874) and Whitaker (1920) in spring migrating through Sicily and it has been reported also by Massa et al. (2015) in autumn from Marettimo island (western Sicily) to Tunisia.

Great Tit Parus major and Blue Tit Cyanistes caeruleus. While the Great Tit has always been observed commonly all over Sicily, included town gardens, the Blue Tit has shown a different ecological niche and has been rarely observed outside woodland. Pantelleria (Sicilian Channel) houses the only European population of the African Blue Tit Cyanistes teneriffae ultramarinus (Fig. 3).

Woodlark Lullula arborea. According to Massa \& La Mantia (2010), the Woodlark seems more or less stable on breeding grounds all over Italy, while migrant birds, once common, today appear scarce; the flocks of hundreds, generally observed and recorded by different authors in southern Italy, are missing today. Massa \& La Mantia (2010) assumed that the Woodlark is less declining, probably due to the fact that, even though in winter it occurs in the same habitats as Calandra Lark and Skylark (both declining), during the breeding season it is linked to clearings and wood edges, habitats less threatened than pseudosteppes. 


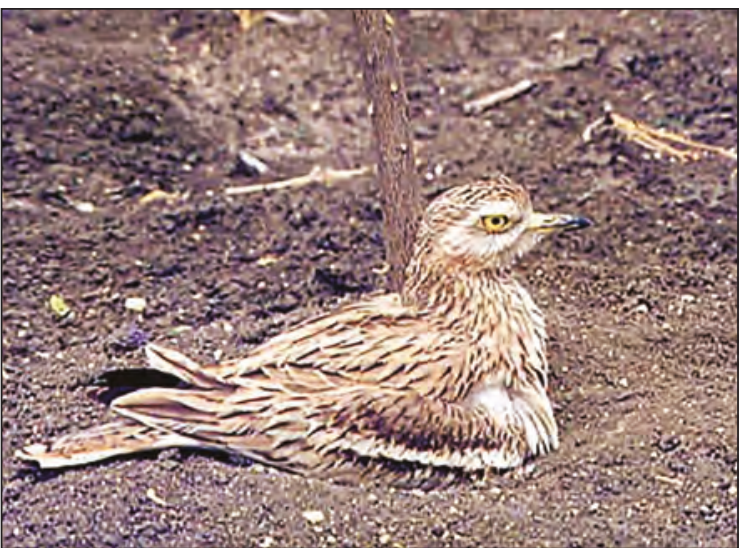

Figure 2. Eurasian Stone Curlew Burhinus oedicnemus. The pattern of plumage of the Sicilian population intergrades between ssp. oedicnemus and ssp. saharae, being limited the black portion of feathers on the body (photo by A. Priolo).

Zitting Cisticola Cisticola juncidis. This small bird is present in the Sicilian territory with two subspecies. On the mainland Sicily $C$. juncidis juncidis is a resident breeder which over the time spam here considered resulted to be stable; it colonized also the Ustica Is. in the 1970s, but disappeared there in 2010 (Massa et al., 2015). On the islands of Pantelleria and Lampedusa the North African subspecies C. juncidis cisticola has been found; this taxon very probably colonized Pantelleria in 1900 because it was not detected by Doderlein (1869-1874), who visited Pantelleria, but it was found in the island by Foschi (1968) and Moltoni (1973). Interestingly, previously absent from Lampedusa (Moltoni, 1970), it was observed more recently (since 1995: Lo Valvo, 2001), but it does not overwinter on the small island (Massa et al., 2015). Overall, populations of the Zitting Cisticola seem to be more fluctuating than stable over long periods.

Sicilian Long-tailed Tit Aegithalos caudatus siculus (Figs. 4, 5). It is a very characteristic and differentiated endemic taxon compared to the conspecifics of peninsular Italy; it is sedentary in Sicily, rather localized in broadleaved thickets, where it shows erratic habits. Throughout the long period here examined, all authors found this species as a rather scarce resident bird. Only in 1901 Whitaker described it as Acredula sicula (presently subspecies of $A$. caudatus), and in 1902 he published a very nice color plate by Henrik Grönvold, at the time illustrator for the British $\mathrm{Mu}$ seum of Natural History, who depicted also other drawings for Joseph Whitaker "The Birds of Tunisia".

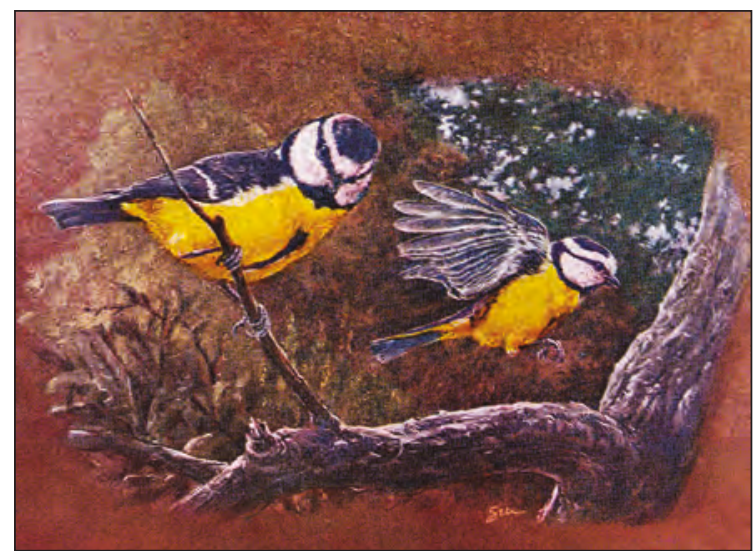

Figure 3. African Blue Tit Cyanistes teneriffae ultramarinus. Pantelleria (Sicilian Channel) houses the only European population of this nice tit, linked to oaks and maritime pines of the small island (drawing by S. Maugeri).

Eurasian Nuthatch Sitta europaea. The Eurasian Nuthatch has still stable populations on natural woodland of north Sicily (from Madonie Mts. eastwards to Peloritani and Etna). The presence on Iblei Mts. (south-east Sicily) reported by Riera (1923), and in the Aidone forestation (central-east Sicily) (Mebs, 1957) has not been confirmed.

European Robin Erithacus rubecula. The Robin was always very common and abundant in Sicily during the winter months, so that the Palermo kids (but also some men) who knew well the territorial behavior of this little bird, in October, when the wintering Robins arrived from north regions, caught one alive and accustomed it to being inside a cage with a semicircular roof. Then, they inserted sticks with mistletoe on the roof and carried around the cage in the gardens of towns and villages. To try to hunt the intruder, the Robins remained entangled on a wand and immediately captured by the young poacher, who was able to capture dozens of them in a day. The poor Robins, plucked and cooked, served as dinner in the houses of a poor proletariat. Despite this bad tradition, the Robin population wintering in Sicily did not diminish over the longtime here considered. Now, fortunately the tradition disappeared.

\section{ii) Declining (dec) and iii) very declining species (vdec)}

A large part of the species are declining (75) or very declining (34). Human-induced environmental 
changes have profoundly affected bird populations. The categories of birds that lost the greatest number of species, in order, are: aquatic, terrestrial of open habitats or pseudosteppes, and seabirds. Humid habitats, as highlighted, have undergone a strong contraction, while the species of open habitats have been strongly negatively affected by the abandon-

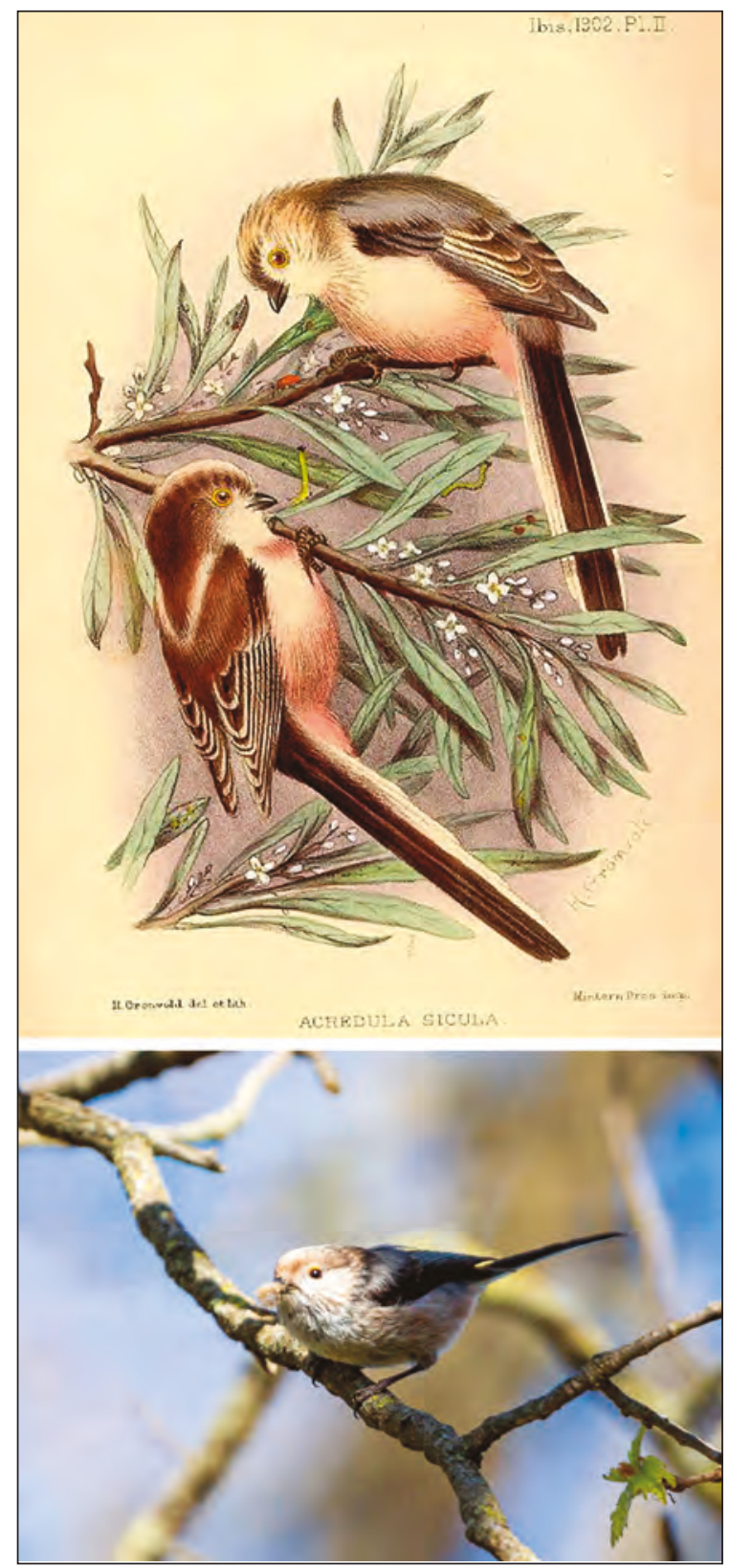

Figures 4, 5. Sicilian Long-tailed Tit Aegithalos caudatus siculus. The drawing by Henrik Grönvold (Fig. 4, above) and one individual in the wild of this very peculiar endemic taxon (Fig. 5, below) (photo by M. Coco). ment of traditional agricultural practices, the introduction of mechanical means and the use of chemical inputs (Massa \& La Mantia, 2007). In this section we discuss of the decline of some representatives, namely: Common Quail Coturnix coturnix, Sicilian Rock Partridge Alectoris graeca whitakeri, Ferruginous Duck Aythya nyroca, European Turtledove Streptopelia turtur, Mediterranean Storm Petrel Hydrobates pelagicus melitensis, Slender-billed Curlew Numenius tenuirostris, Ruff Calidris pugnax, Eurasian Woodcock Scolopax rusticola, Egyptian Vultur Neophron percnopterus, Red Kite Milvus milvus, Woodchat Shrike Lanius senator and Larks Alaudidae.

Common Quail Coturnix coturnix. Historically it was subjected to an important hunting activity, and in some cases it had also an economic support for the local people. In Messina in the late $19^{\text {th }}$ century, the spring chronicles report the capture of over one million individuals (Giglioli, 1889-1890). It was abundant, during the migration, also on the small islands of Pantelleria (Iapichino \& Massa, 1989) and Ustica (Ajola, 1959); presently this phenomenon is not so evident, the migration flows are present but largely reduced. Even if the breeding area has remained substantially unchanged, today the territorial song of very few males is detected over the suitable habitat, where once they were numerous (Surdo, 2019).

Sicilian Rock Patridge Alectoris graeca whitakeri (Fig. 6). It was formerly widespread throughout hilly and rocky areas of the island. Presently, it is rare or present at very low densities in hills, most populations are confined near mountain slopes or other not very accessible places, as stony ground and volcanic surfaces (Etna). The only thriving populations of this endemic taxon live inside the Natural Parks.

Ferruginosus Duck Aythya nyroca. Common, or very common in the past, recently rare, in Sicily as in all the Palaearctic Region; it has become rare throughout the range since the 1950 s, while formerly it was one of the most common species of ducks (Robinson \& Huges, 2006). Even today an important spring migratory influx interests the coast of Sicily in front of Sicilian channel, mainly in the Gulf of Gela, but the numbers are much smaller than in the past (Campo et al., 2001). There is no historical information on the presence of breeding pairs. However, it is an elusive species and breeds in wetlands rich of vegetation; thus, it is easily unnoticed. 


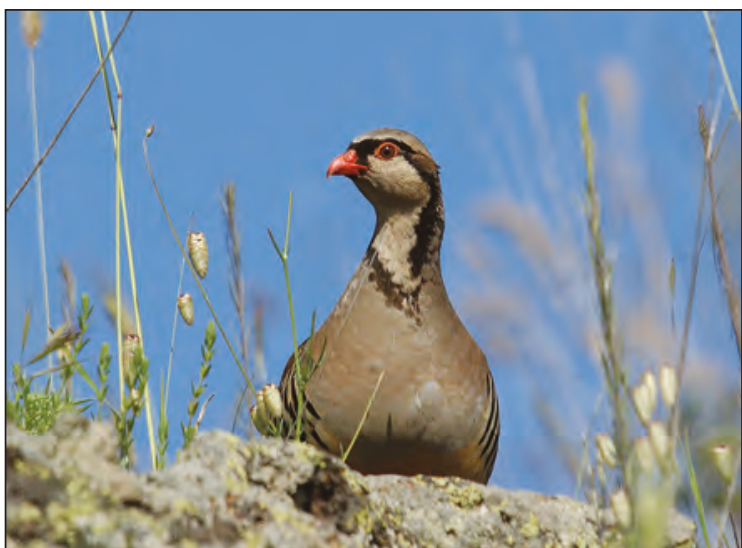

Figure 6. Sicilian Rock Partridge Alectoris graeca whitakeri. This very peculiar partridge, named in 1934 by Guido Schiebel in honour to Joseph Whitaker, compared to alpine individuals, is characterized by its smaller size, interrupted black collar, dirty white throat and ochreous ear coverts (photo by T. Puma).

Coot Fulica atra. Abundant in the past, when huge numbers were reported in winter from a lot of inner lakes and coastal wetlands. There was a considerable decrease of numbers in many European countries during the $19^{\text {th }}$ century (Hagemeijer \& Blair, 1997). The negative trend continues, becoming more pronounced in recent years, affecting also wintering waterfowl in general.

European Turtle-dove Streptopelia turtur. Formerly it was an abundant migratory, largely hunted in spring. At the end of the $19^{\text {th }}$ century, the hunters at Messina took thousands (Giglioli, 1889-1890); in spring, along the south coast of Sicily formerly they arrived in flocks, usually up to 50 individuals, sometimes 1000 in a day in some areas (Iapichino \& Massa, 1989). Nowadays, the Turtle Dove has decreased dramatically, like in the rest of Italy and in the other two Mediterranean peninsulas (BirdLife International, 2017).

Mediterranean Storm Petrel Hydrobates pelagicus melitensis (Fig. 7). Formerly common in the Messina Strait and generally widespread in the Sicilian waters (Doderlein, 1869-1874), presently reduced to small colonies scattered in the islands surrounding Sicily. Only the population of the Marettimo Is. is numerous and overall consists of ca. 2,000 pairs (Albores-Barajas et al., 2012). In total, Mediterranean basin hosts 8,500-15,200 pairs of the ssp. melitensis, an unsubstantial proportion of the whole European population, estimated between 438,000 and 514,000 pairs

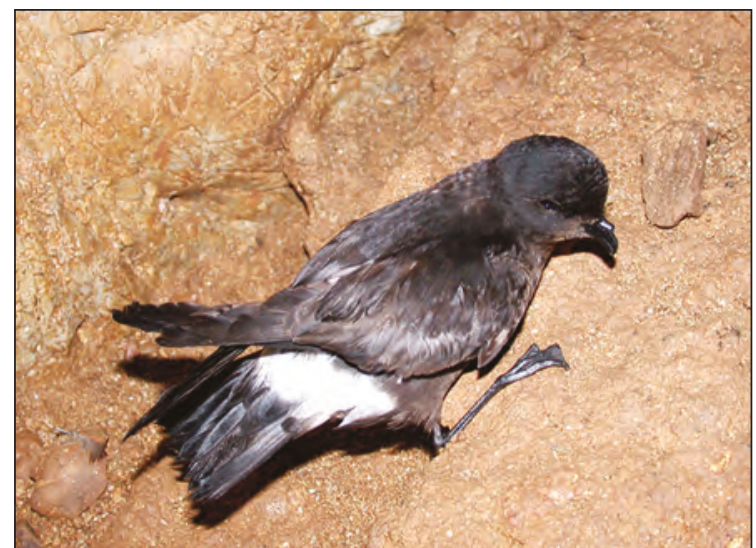

Figure 7. Mediterranean Storm Petrel Hydrobates pelagicus melitensis. Characterized by a bigger size than the Atlantic Storm Petrel, the Mediterranean taxon is rare and scattered on small islands, and, differently from the Atlantic one, their small populations are endangered (photo by B. Massa).

(BirdLife International, 2017). A major factor limiting the breeding distribution of this subspecies is the presence of safe colony sites on islands, that is why the Mediterranean populations are so depleted (Massa \& Borg, 2018). Even if a possible mixing of Atlantic (H. p. pelagicus) and Mediterranean ( $H$. p. melitensis) populations occurs in winter, Atlantic individuals have never been found during the breeding season in the Mediterranean; we are convinced that they should be treated as separated species.

Slender-billed Curlew Numenius tenuirostris. It currently is probably one of the rarest birds in the Palaearctic Region; according to the International Union for Conservation of Nature (IUCN, 2017) it is one of the most 'Critically Endangered' European bird species. The last observations date back to 1999. The only evidence of its breeding site dates back to the first years of 1900 and concerns the area of Tara, in the region of Omsk (South-West Siberia, East of Ural Mts). Once it wintered regularly in the Mediterranean and was rather frequent also in Italy, more common than the Whimbrel Numenius phaeopus; Morocco was a favoured wintering area, last flights of more than 100 individuals were observed in January 1964 in the lagoon of Puerto Cansado, Khnifiss (500-800 ind.) and in December 1974 in the Oued Chebeika (123 ind.). Since 1995, in the last regular wintering site in Morocco, Merja Zerga, the Slender-billed Curlew was no longer observed (Gretton et al., 2002). In Sicily formerly was also 
very common as migrant or wintering bird until the half of 1900; later on it disappeared.

Golden Plover Pluvialis apricaria. Following former authors, it was very abundant and widespread in the wide alluvional plains of the island, now it is in evident decline. It is negatively affected by the intensive use of the soil and the human presence. The degradation and reclamation of temporary wetlands had an important role on the population decline.

Ruff Calidris pugnax. The Ruff is considered declining in Europe (BirdLife International, 2017) and over the long-term period here examined we notice it as a very declining migrant bird in Sicily. This is the reason of a serious debate about the necessity to close the hunting to this wader bird.

Eurasian Woodcock Scolopax rusticola. Even if BirdLife International (2017) considers the Woodcock to be stable in Europe, actually it decreased in the long period here considered, very probably due to the decline in the north-west countries, where the birds wintering in Sicily are breeding. It is one of the most hunted birds in Italy during the winter season.

Egyptian Vulture Neophron percnopterus (Figs. $8,9)$. This necrophagous bird, already depicted by Cupani (1713), was considered rare by Doderlein (1869-1874), later, Iapichino \& Massa (1989) recorded twenty breeding pairs, while more recently its population has been estimated as much as 7-9 breeding pairs (Andreotti et al., 2020). Even if it is globally threatened in the Mediterranean, and is a transaharan migrant, wintering in tropical Africa, its high and low peaks of Sicilian population could be dependent from bird fluctuations more than on other factors. However, it seems really decreasing; we would list it as fluctuating just to send a message of hope for a future recovery, but we believe that its Sicilian population is recently endangered and very declining.

Red Kite Milvus milvus. It was a common resident raptor, estimated as much as 90 pairs by Massa \& Schenk (1983), 40 pairs by Iapichino \& Massa (1989), 10-12 by Cortone et al. (1994) and 5-6 in 2006 by Ientile $\&$ Massa (2008). Presently, it is very rare, and probably only $2-3$ pairs remain to breed in the island.

Woodchat Shrike Lanius senator. It was formerly widespread in Sicily. According to Benoit (1840) it was the most common species around Messina. It usually bred on tree crops (citrus, almond and olive groves). In the last twenty years it has strongly decreased, probably for the excessive use of pesticides and for the disappearance of dried almonds, once part of their breeding habitat (Massa \& La Mantia, 2007).

Larks Alaudidae. They represent an emblematic group, negatively involved by agricultural transformations. Formerly very abundant, and traditionally hunted or captured and caged as melodious singing birds (e.g., Calandra Lark Melanocorypha calan$d r a$ ). In the last thirty years, Calandra Lark and Short-toed Lark Calandrella brachydactyla became very rare and local (Massa, 1985; Massa \& La Man-

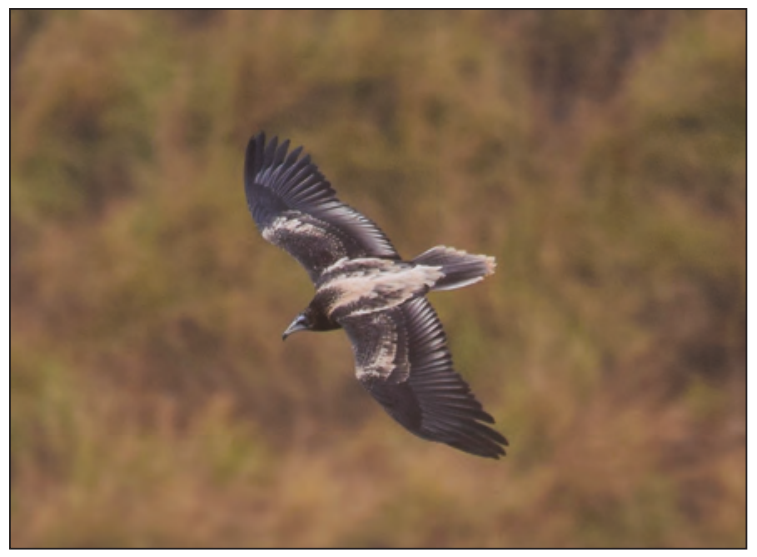

Figures. 8, 9. Aegyptian Vulture Neophron percnopterus. Adult (Fig. 8, left) and young (Fig. 9, right) individuals of the remaining breeding Sicilian population; throughout one hundred and fifty years this bird resulted to be declining or more or less fluctuating and this gives us hope for a future recovery (photos by A. Cairone). 
tia, 2010; Surdo, 2019). The Skylark Alauda arvensis in the past was abundant during the migration and in winter months; many authors between early 1800 (Rafinesque, 1814; Doderlein, 1869-1874) and early-mid 1900 (Whitaker, 1905, 1920) reported a massive passage in the Gulf of Palermo, estimated as much as some million of individuals, subjected to a systematic hunting. The strong decline in this group of birds, reflecting a general decline in all Europe, begun in 1970s and is still ongoing (Massa \& La Mantia, 2010; BirdLife International, 2004, 2017).

\section{iv) Increasing (in) and v) very increasing (vin) species}

Among the 47 species that resulted increasing or very increasing over the long period examined we selected the following fifteen for a wider discussion: Marbled Teal Marmaronetta angustirostris, Woodpigeon Columba palumbus, Eurasian Collared-dove Streptopelia decaocto, Pallid Swift Apus pallidus, White Stork Ciconia ciconia, Cattle Egret Bubulcus ibis, Northern Gannet Morus bassanus, Ruddy Turnstone Arenaria interpres, Audouin's Gull Larus audouinii, Bonelli's Eagle Aquila fasciata, Booted Eagle Hieraaetus pennatus, European Bee-eater Merops apiaster, Peregrine Falcon Falco peregrinus, Eurasian Blackbird Turdus merula and European Greenfinch Chloris chloris.

Marbled Teal Marmaronetta angustirostris. Considered accidental by Doderlein (1869-1874), irregular migrant, in some years not uncommon, by Whitaker (1920) and again accidental by Iapichino \& Massa (1989). In 1999 for the first time it bred in western Sicily, and in the following years also in eastern Sicily more or less regularly (Sciabica, 2001, 2004; Corso, 2005; Ientile \& Massa, 2008). The recent colonization to Sicily by a small population may be the result of the recently increased wintering and breeding numbers in Tunisia (Isenmann et al., 2005).

Woodpigeon Columba palumbus. In former years the Woodpigeon was a species linked to Sicilian woodland, but since 2000 s, probably due to the rise of wintering birds, this species has colonized also towns and villages, living very commonly in the gardens and even in the trees bordering city streets; it colonized also some small islets surrounding Sicily (Ustica, Egadi Is., Pantelleria: Massa et al., 2015). In addition it regularly breeds precociously and may nest three or four times per year (La Mantia, 1994; pers. obs.).

Eurasian Collared-dove Streptopelia decaocto. Considered accidental until 1988 (Iapichino \& Massa, 1989), it colonized Sicily in the late 1980s, both with individuals from captivity and with wild individuals. The new wave of expansion of this species took place in the 1990s and has affected southwestern Europe and North Africa. We cannot establish the origin of Sicilian individuals; today also the small islands surrounding Sicily were colonized by this bird, including Pantelleria and Pelagie Islands.

Pallid Swift Apus pallidus. It has recently increased, mainly in urban environments, but also in some coastal cliffs. In urban environments there are apparently mixed colonies with the Common Swift Apus apus, which in turn is declining; the most suitable period to estimate the consistency of the Pallid Swift is from August onwards, when the Common Swift has left Sicily for the African winter quarters; the Pallid Swift remains still until September-October and carries out a second brood.

White Stork Ciconia ciconia. After the first cases of breeding in the 1990s, this species has undergone a consistent demographic growth in Sicily, probably by individuals belonging to the Tunisian population, recorded as increasing by Azafzaf (2002). Currently between 70 and 80 pairs are breeding in the island (Zafarana et al., 2020). Interestingly, the breeding population is largely sedentary, and also some migrant individuals are presently wintering on the island.

Cattle Egret Bubulcus ibis. Formerly accidental, consistently with the trend in Italy and most of the Mediterranean countries, the Cattle Egret has colonized Sicily; it winters regularly since the late 1990 s, presently with huge numbers, and since 2001 has regularly bred in the island (Ciaccio, 2004; Surdo, 2019).

Northern Gannet Morus bassanus. It was so rare in the Mediterranean that Doderlein (1869-1874) did not list it at all; however, in 1882 he obtained the first specimen (Doderlein, 1883). According to Whitaker (1920) the Gannet was 'of occasional occurrence, but probably not as uncommon as is generally supposed'. Following the increase of north European populations, this seabird after the mid of 
the past century became much more common as wintering in the Mediterranean, Sicily included (Iapichino \& Massa, 1989; Corso, 2005). Presently it is a scarce wintering bird, which may be often encountered offshore.

Ruddy Turnstone Arenaria interpres. This wader was formerly rare and irregular migrant, but presently its populations are much more common in winter; according to Surdo (2016) winter numbers at Trapani saltpans represent almost $50 \%$ of the entire average population that wintered in Italy in the years 2006-2010.

Audouin's Gull Larus audouinii. While Doderlein (1869-1874) considered it as an accidental gull, Whitaker (1920) stated is as 'resident'; later on it has been considered a scarce migrant bird, rare in winter (Iapichino \& Massa, 1989; Corso, 2005) up to 2010 , when it has colonized the small island in front of Vendicari Nature Reserve (eastern Sicily) (Ientile et al., 2016).

Bonelli's Eagle Aquila fasciata (Fig. 10). This is an interesting case of a large predator that has increased in the last two decades, while formerly it was a rare resident breeder and in more recent years its population did not exceed 20 pairs. Presently the number of occupied territories resulted to be ca. 50, and it seems that the population has grown in the last fifteen years. Following Di Vittorio et al. (2019), this might be dependent from a change in the diet, presently consisting mainly of birds (57.6\%), of which most were pigeons (Rock Dove and Woodpigeon, 38.4\%) and corvids (three species, $18.5 \%$ ), while rabbits represented a lower percentage $(37.1 \%)$. Interestingly, this change of diet has occurred when the population of rabbits declined dramatically, whereas Woodpigeons have increased notably and a huge numbers of feral pigeons (the domestic race of Columba livia) are more and more widespread all over Sicily.

Booted Eagle Hieraaetus pennatus. This small eagle was unknown in Sicily until the half of the last century; later a few individuals occurred in north Italy and rarely in Sicily (Iapichino \& Massa, 1989), but since the years 2000s it became a regular visitor of the island (Baghino et al., 2007). Presently its winter presence is normal, and it may be observed not rarely flying over the town buildings.

European Bee-eater Merops apiaster. The Beeeater was a fairly frequent migratory bird, and very few cases of breeding in Sicily were known (Do- derlein, 1869-1874; Whitaker, 1920; Iapichino \& Massa, 1989); in the last decades it has become very common and numerous and has formed small colonies scattered throughout the island. A substantial population growth occurred at the end of the 1990s, which resulted to be parallel to that verified by various ornithologists in the rest of Italy. Probably its general increase in populations was due to the increase in beekeeping, being a specialized predator of Hymenoptera, mainly bees (Grenci et al., 1997; Massa \& Rizzo, 2002).

Peregrine Falcon Falco peregrinus. The first census of this raptor was carried out in Italy in the early 1980s (Schenk et al., 1985); the total number of breedings pairs in Sicily resulted as much as 150 . Twenty years later Corso (2005) estimated ca. 250 pairs and Ientile \& Massa (2008) ca. 300 pairs in the island. The increase very likely occurred both thanks to the colonization of new sites, not previously occupied by this raptor, and to a smaller distance between pairs, which in some cases was reduced to a few hundred meters; in the latter case breeding sites were particularly frequented by spring migrant passerines.

Eurasian Blackbird Turdus merula. This songbird was formerly very common as migrant, but its numbers diminished like the other thrushes. Conversely, while formerly it was a scarce breeder, presently it is a common breeding bird, and there are many observed cases of three consecutive reproductions in the same year.

European Greenfinch Chloris chloris. As as reported below for the Chaffinch, this finch, once uncommon breeder in Sicily, in the last decades has increased noticeably, becoming one of the commonest birds in the town gardens.

\section{vi) Fluctuating species (flu)}

In nature, population fluctuations are normally phenomena due to different factors, as the immigration, the birth and mortality rate, etc. In particular fluctuations are easily observed in the migratory birds, that exploit different geographical areas for breeding and wintering. Within the phenomenon of the fluctuation there is also the sedentarization of some species, normally only migrant breeders, that for some reasons change their migration habits, often only partially. We report here some examples of Sicilian fluctuating birds. 


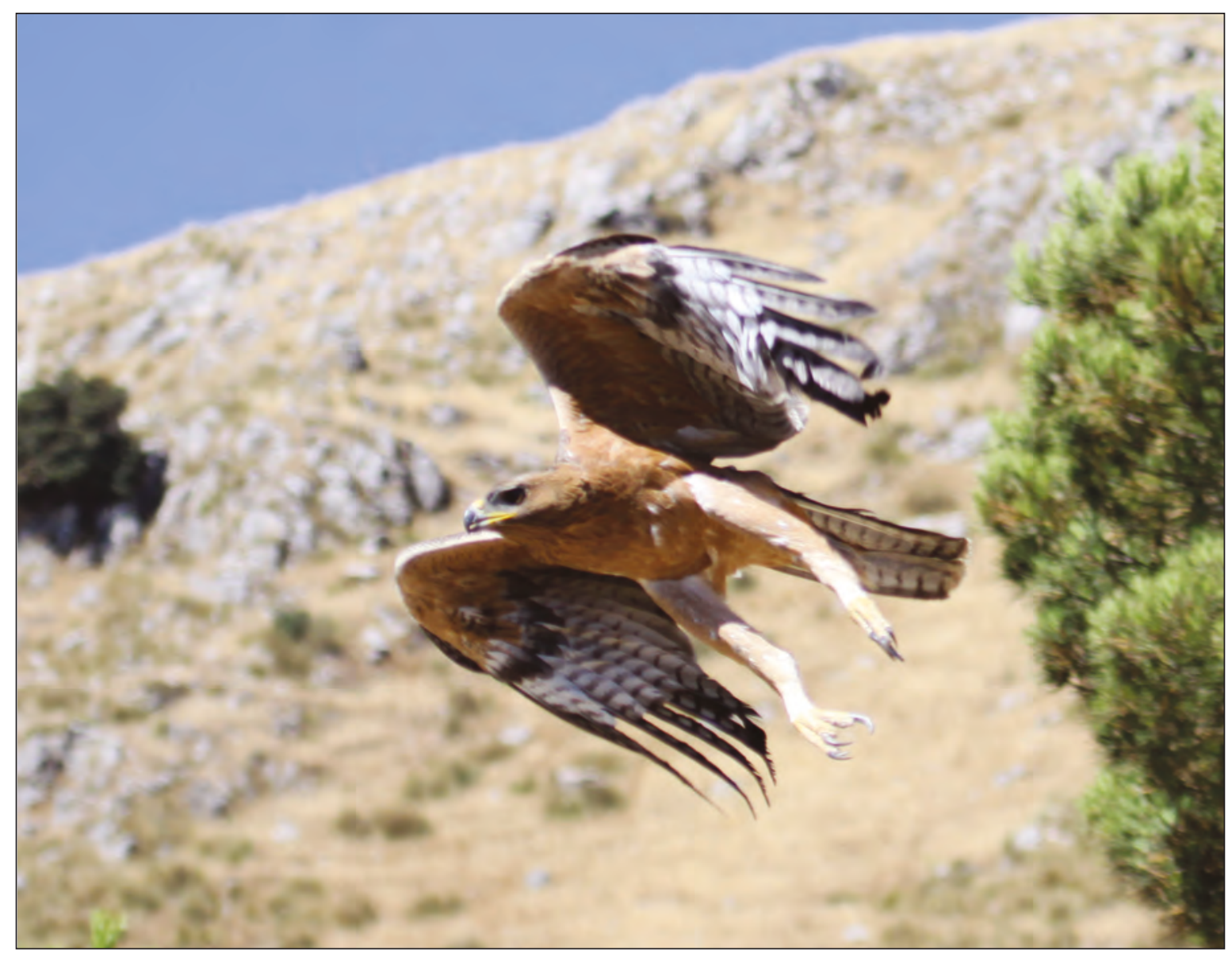

Figure 10. Bonelli's Eagle Aquila fasciata, young. It has increased in the last decades, and its population has grown in the last fifteen years to ca. 50 pairs, a trend opposite to that observed in the rest of its distribution (photo by M. Di Vittorio).

Glossy Ibis Plegadis falcinellus. It was common and bred in the marshes of eastern plains of Sicily in the half of 1800 , but, certainly due to drainage of wetlands, its numbers were only limited to migrating birds, still common in the early 1900s, later scarce; however, in the last decades it has returned to breed in humid habitats of some Sicilian Nature Reserves. This possibly is the result of a protection granted to this species in the last years.

Cormorant Phalacrocorax carbo. According to Doderlein (1869-1874) the Cormorant was very common and bred in Sicily, following Whitaker (1920) it was still very common but no more breeding, and according to Iapichino \& Massa (1989) its wintering Sicilian population was declining; it increased again in the 2000 s, when it returned to breed in some limited Sicilian marshland.

Black Stork Ciconia nigra. This bird was con- sidered rare in Sicily by ornithologists of 1800 and first years of 1900, scarce by ornithologists of late 1900 s, early 2000s, and presently. Populations of this Stork visiting Italy are increasing (Brichetti \& Fracasso, 2018), but in the last years its status in Sicily remained unchanged; it is uncertain if it should be classified among fluctuating or increasing species in Sicily.

Kentish Plover Charadrius alexandrinus. This is a different case from the previous ones. Really this species, in accordance with Doderlein (18691874), was rare and only migrant, while Whitaker (1920) found it as scarce breeder, Massa \& Schenk (1983) estimated a population of 400-500 pairs, while Surdo \& Matteucci (2016) observed a diminution of $70 \%$ of the Sicilian population, that now is very scarce. Maybe these high and low peaks over one hundred and fifty years are better explained with population fluctuations. 
Little Tern Sternula albifrons. This species was formerly common or very common, at least as migrant bird, rare (Doderlein, 1869-1874) and common as breeder (Whitaker, 1920), while in more recent years it became scarce both as migrant as well as breeder bird. Its populations are typically fluctuating and from year to year colonies may vary much in numbers.

Little Owl Athene noctua. This Owl has been considered common breeder by all the authors except for Whitaker (1920), who considered it scarce. This possibly might depend on a different personal significance of the terms 'common' and 'scarce' or on a species fluctuation. We would highlight that in the former times the game bag of hunters contained species that today are considered inedible (and also protected by the law), but the hunger caused a very serious hunting to these birds. For example since the 1800 up to half of 1900 the hunters took hundreds if not thousands of Eurasian Scops Owls Otus scops during migration seasons and, cooking them, obtained from their meat a sauce for pasta.

Black Kite Milvus migrans. Interestingly, the Black Kite was very rare in the half of 1800 , and rare in 1920 as passage migrant, while it was considered common as passage migrant and breeder in the late 1980s, also scarcely wintering in the early 2000s; but, even if its migrant populations remained stable in the last years, very likely no individuals are more breeding. It is also interesting to highlight that while Cupani (1713) depicted the Red Kite Milvus milvus, he did not left any drawing of the Black Kite (Priolo, 1996); the rarity of this latter species was an objective fact. There are two considerations for this species: i) migrant populations were increasing through one hundred and fifty years from very rare to common; ii) for about twenty five years the Black Kite colonized the island, but later it was slowly decreasing and disappeared as breeder.

Lanner Falcon Falco biarmicus feldeggii (Fig. 11). It is one of the most elusive species among falcons. While Doderlein (1869-1874) does not mention it at all, according to Priolo (1996) it was depicted by Cupani (1713); thus, it was present in Sicily but never observed by Doderlein, and considered accidental by Whitaker (1920). However, we consider this species an emblematic case of bird fluctuation. In the first years of the past century it was abundantly captured by people who hunted larks in October in Apulia (south Italy); in the period 1905-1928 ca. 450 specimens are reported by Arrigoni degli Oddi (1929), probably coming from Balkans following lark flocks migrating towards Italy. Sicilian census of this species gave data very fluctuating: 60 pairs by Iapichino \& Massa (1989), but 90 by Massa et al. (1991), 100-120 by Corso (2005) and again 60 by Di Vittorio et al. (2017). It seems that the Lanner Falcon has been a regularly fluctuating species and the recent diminution may lie within its high and low peaks of abundance. In addition, its elusive habits avoid every accurate counting of breeding pairs.

Thrushes Turdus spp. Apart from the Song Thrush Turdus philomelos, which is objectively decreasing in Sicily as well as in all Europe (BirdLife International, 2017), the Redwing Turdus iliacus, the Fieldfare Turdus pilaris and the Ring Ouzel Turdus torquatus were fluctuating in Sicily over a period of one hundred and fifty years. They were regular wintering, even if scarce or rare, in the half of 1800 , irregular in the early 1900 s, regular wintering in the late 1900 s, and again irregular wintering in 2000s. Very likely numbers wintering in the Mediterranean area depend on the status of breeding populations in central Europe.

Spanish Sparrow Passer hispaniolensis and Italian Sparrow Passer italiae (Figs. 12, 13). Sparrows have often undergone fluctuations. This happened also in Sicily, but it was more evident in the small island of Ustica. Doderlein (18691874) wrote that the Sparrows were completely missing on the Ustica island, as they were deliberately eliminated by the farmers. However, between the end of the $19^{\text {th }}$ century and the beginning of the $20^{\text {th }}$ century, the Italian Sparrow Passer italiae was present in the island; indeed at the Civic Museum of Zoology in Rome two specimen skins of males captured on the island on 13.VIII.1896 and 18.IV.1900 are preserved (Massa, 1973). In the half of 1900 both Steinbacher (1955) and Ajola (1959) reported the absence of Sparrows. The recolonization of the island by the Spanish Sparrow probably dates back to the end of the 1960 s of the $20^{\text {th }}$ century, and at the beginning of the 1970s the population was very abundant. During the last thirty years, the species has undergone periods with significant 
demographic growth, followed by periods of decrease (Iapichino \& Massa, 1989). Interestingly, after the colonization of the Spanish Sparrow, the Tree Sparrow Passer montanus, once abundant in Ustica (Ajola, 1959), rapidly decreased in number (Massa et al., 2015).

Chaffinch Fringilla coelebs. The Chaffinch may be considered an iconic fluctuating finch: Doderlein (1869-1874) considered it as very commonly wintering, but rarely breeding, Whitaker (1920) also very common in winter but scarcely breeding, while Iapichino \& Massa (1989) recorded it as common both as breeding and wintering. This status was maintained till the early 2000 s, but later both migrating and breeding populations declined, in some areas dramatically. For example, in the town of Palermo, the Chaffinch was a common breeding bird until the first years of $2000 \mathrm{~s}$, but now it is completely missing; conversely, the Green Finch Chlo- ris chloris, once a rare breeder finch, now is one of the most common species in Palermo. Concerning most species of finches, the illegal mist netting for commercial purposes is still an important cause of their temporary decline, that may simulate a fluctuation.

Red Crossbill Loxia curvirostra (Figs. 14-16). This finch is linked to coniferous trees; in Sicily a sedentary population lives on Mt. Etna, linked to Pinus laricio. Further, some irruptive populations colonize irregularly the island, generally only during winter months, but in some cases they were breeding for a variable number of years. This may explain the fluctuating status. However, the resident population of Etna, numerically stable, is characterized by less colored individuals, which have a bigger and stouter bill, considered and adaptive character to the bigger cone scales of Pinus nigra (Massa, 1987b).

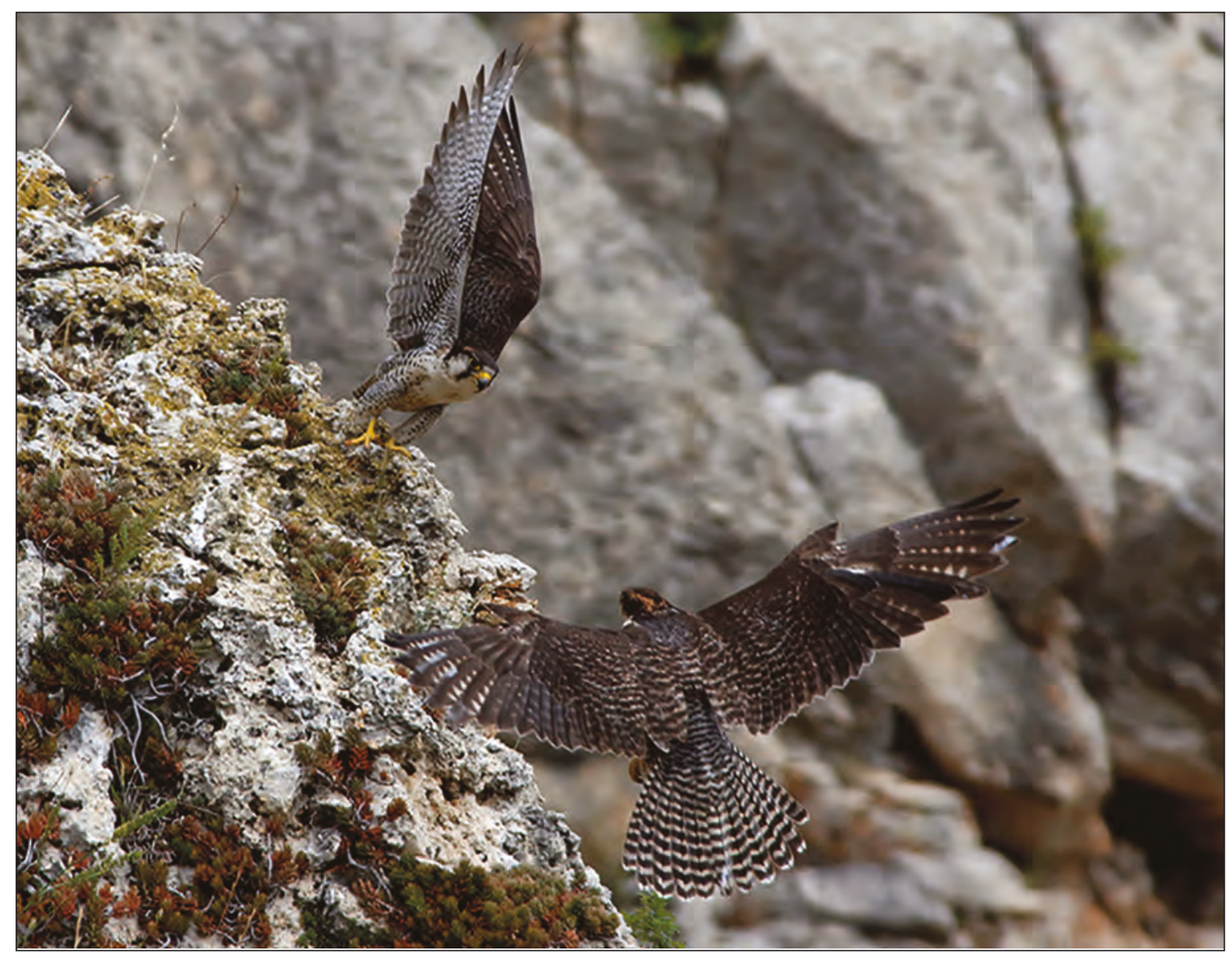

Figure 11. Lanner Falcon Falco biarmicus feldeggii. It seems that the Lanner Falcon has been a regularly fluctuating species and the recent diminution may lie within its high and low peaks of abundance (photo by A. Nardo). 

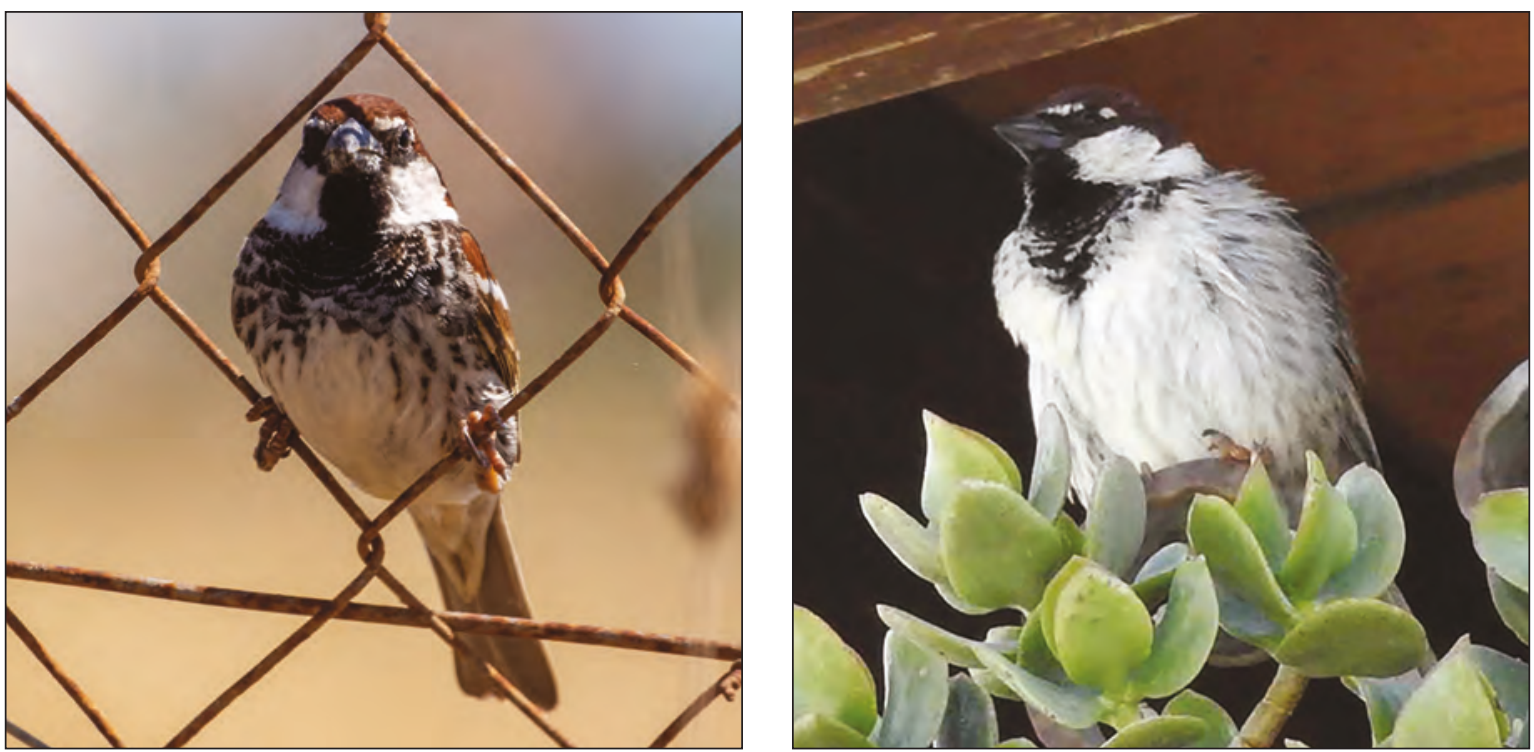

Figures 12, 13. Spanish Sparrow Passer hispaniolensis (Fig. 12, left) and Italian Sparrow Passer italiae (Fig. 13, right). The taxonomy of sparrows living in Italy is very intricate and many authors worked on it trying to propose an arrangement. The two images, both from Sicily, seem morphologically belonging to two species (photos by A. Barbera and B. Massa).

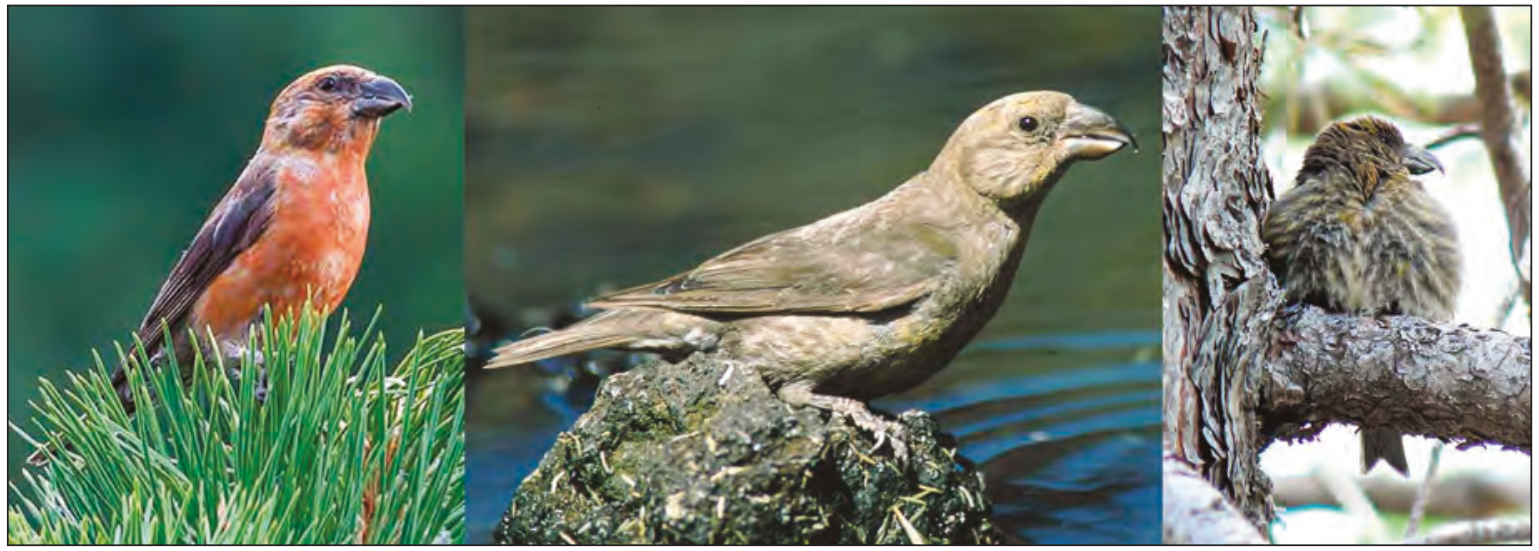

Figures 14-16. Red Crossbill Loxia curvirostra. The individuals of the resident population of Etna, as well as those living in Calabria (south Italy), are linked to Pinus nigra and are characterized by a less colored plumage and a stouter bill than north Italian ones (male: photo by T. Puma; female: by A. Ciaccio; young: by B. Massa).

\section{vii) Extinct species (Ex)}

Extinction is a natural phenomenon, on the causes of which there is much debate (bad genes or bad luck? Raup, 1993). However, according to a large number of testimonies, the decline and extinction of many species is currently caused by human being. After a visit to Sicily, in his "Homage to Santa Rosalia", today considered the seminal paper on the biodiversity concept, the ecologist Hutchin- son (1959) wrote that man had reduced diversity by a rapidly increasing tendency to cause extinction of supposedly unwanted species, often in an indiscriminate manner; and he believed that a limited reversal of this process should have occurred when man would become aware of the value of diversity no less in an economic than in an aesthetic and scientific sense. The current sixth mass extinction is mainly caused by human-induced factors, such as the isolation and habitat loss. 
Eleven resident species became extinct in Sicily: Black Francolin Francolinus francolinus, Western Swamphen Porphyrio porphyrio, Little Bustard Tetrax tetrax, Common Bottonquail Turnix sylvaticus, Eagle Owl Bubo bubo, Griffon Vulture Gyps fulvus, Lammergeier Gypaetus barbatus, Black Woodpecker Dryocopus martius, European Green Woodpecker Picus viridis, Lesser spotted Woodpecker Dryobates minor, and Bearded Reedling $\mathrm{Pa}$ nurus biarmicus. In addition, one migrant species, once very common is Sicily, the Slender-billed Curlew Numenius tenuirostris, has very probably become globally extinct. Further, one species introduced in 1800, the Pheasant Phasianus colchicus, was already considered extinct by Doderlein (18691874), and, as reported above, also the Demoiselle Crane Anthropoides virgo was formerly breeding on Lampedusa Is. and presently it is absent from all the Mediterranean area.

The Black Francolin Francolinus francolinus has been the subject of a paper by Lilford (1862), who considered it to become extinct in Sicily. Really, it was an alien species, introduced by Greeks in the $13^{\text {th }}$ century (Oriani, 2014); Forcina et al. (2015) demonstrated that Sicilian Francolins were imported from central Asia and are genetically similar to the population considered belonging to the subspecies asiae.

About the Western Swamphen Porphyrio porphyrio the extinction dates back to ca. 1960; already Whitaker (1920) wrote that it was threatened with extinction, owing to the general reclaiming of marshy lands, and some years before (Whitaker, 1899a, 1899c) published two papers, one in Italian, the other in English, with many interesting remarks on the habits of this beautiful bird, that he reared in his garden at Palermo (Villa Malfitano). In the years 2000s a project of reintroduction has been carried out, with very interesting and fruitful results; presently the species is distributed in many marshes, also very small, all over Sicily (Ientile \& Andreotti, 2003; Ientile \& Massa, 2008).

The Little Bustard Tetrax tetrax was a common resident bird in the half of 1800 (Doderlein, 18691874), but Whitaker (1920) wrote that it appeared to be much rarer; its extinction dates back to ca. 1960 , very likely due to more and more reduced areas of extensive sowable ground, and to the hunting. Its bad status in Italy is serious, because only the Sardinian population still remains, being the south Italian one (living in Apulia) reduced to few individuals (Brichetti \& Fracasso, 2018).

The Common Bottonquail Turnix sylvaticus (Fig. 17) became extinct in Sicily around 1920. Its status in the Mediterranean is dramatic, presently only a small population survives in Morocco and may be classified on verge of extinction (Gutiérrez Expósito et al., 2011). Violani \& Massa (1993) on the basis of the significant biometric differences observed between the Mediterranean Bottonquail (ssp. sylvaticus) and the other two subspecies geographically closer to it, lepuranus from the sub-Saharan area and dussumier from the Asia and Myanmar, consider that the hypothesis that these are separate species is sustainable, also in consideration of the long time interval since the last possible gene exchange. The loss of this species from the Sicily is one of the heaviest effects of habitat destruction and reclamation by man.

The extinction of the Eagle Owl Bubo bubo was the subject of a paper by Sarà et al. (1997); the reasons of the disappearance of this big owl are not known.

Concerning the Griffon Vulture Gyps fulvus, extinct in 1965 (Priolo, 1966), it has been reintroduced with good results in the same area where the last colony lived (Mt. Nebrodi), and presently the colony consists of ca. 150 individuals. Its extinction was caused by the use of poisonous baits, but the populations formerly abundant was gradually declining in the half of the past century.

The extinction of three species of woodpeckers, Black Woodpecker Dryocopus martius, European Green Woodpecker Picus viridis and Lesser spotted Woodpecker Dryobates minor has been deeply examined by La Mantia et al. (2015) who concluded that they might recolonize Sicily spontaneously from Calabria, whose distance of just three kms is not an important barrier for them; Pulvirenti (2015) indeed recorded the observation of one individual of the Black Woodpecker in the Peloritani Mts. Very probably they became extinct for the former reduction of broadleaved forest cover.

The Bearded Reedling Panurus biarmicus was a former resident breeder at Biviere di Lentini, a marshland whose drainage started on the early 1900 and was concluded in the 1950s. Benoit (1840) listed it as fairly common at Lentini, Doderlein (1869-1874) supposed it was more numerous in 
winter and partially migrant, Riera (1923) listed it as scarce at Lentini, while Stresemann (1943) did not find it there. Last known specimen from Lentini dated 1907, but probably it became extinct between 1930s and 1940s (Orlando, 1958). The extinction of this small passerine seems dependent from the Lentini marshland reclamation, in turn carried out to fight the malaria spreading in Sicily.

\section{CONCLUDING REMARKS}

The status of birds in Sicily today is quite worrying, a very high number of species, 109 (38.5\%) mostly breeding, has decreased; this condition falls under the general Italian status, in which $30.4 \%$ of the breeding species have a bad status (Gustin et al., 2019). Although Sicily was the first Italian region to adopt a law that established an adequate number of Parks and Nature Reserves and which adhered to the Habitat and Birds Directives, with the delimitation of numerous SCIs and SPAs, the objective that was to be achieved, that is, to slow down the loss of biodiversity, has bitterly failed.

The risk is that, continuing at this rate, the number of extinct species will continue to rise. The primary cause of this is the degradation and subtraction of habitat for fauna and this applies not only to birds, but also to mammals and invertebrates, for which a dramatic decrease has been observed over the last dozen years.

Moreover, paradoxically, wishing to develop the exploitation of renewable energies, without a prior general project, numerous wind farms and photovoltaic systems have been installed that are causing direct and indirect mortality and further remove suitable habitat for many species, some of which are already considered at risk at European level.

These problems, combined with the indifferent attitude of a large part of the population towards the environment and nature in general, is causing a recent and unprecedented loss of biodiversity.

Appendix 1. The unknown manuscript on Birds of Sicily by Joseph Whitaker

Here we present the transcription of the eighteen pages of the Whitaker manuscript; each Figure cor- responds to one page. In bold the current name of the species, followed by the name listed in the manuscript with the original annotations. Pages are transcribed following the original sequence in the manuscript and the nomenclature of Dresser (1881).

Page 1 (Fig. 18)

Turdus viscivorus Linnaeus, 1758

Turdus viscivorus $\mathrm{L}$. Winter migrant, fairly abundant, a few breed in the higher and more mountainous regions. Nests with eggs and young obtained at Ficuzza. Turdus philomelos C.L. Brehm, 1831

Turdus musicus $\mathrm{L}$. Winter migrant in vast numbers, of regular passage, spring and autumn.

Turdus iliacus Linnaeus, 1758

Turdus iliacus L. Winter migrant inland, but not at all common (irregular), many more often seen in some years than in others.

Turdus pilaris Linnaeus, 1758

Turdus pilaris L. Winter migrant inland, uncommon (irregular), many more often seen in some years than in others.

Turdus merula Linnaeus, 1758

Turdus merula L. Resident and migratory. Breeds in higher districts.

Turdus torquatus torquatus Linnaeus, 1758

Turdus torquatus $\mathrm{L}$. Winter migrant of irregular passage and somehow rare.

Turdus torquatus alpestris (C.L. Brehm, 1831)

Turdus torquatus alpestris (C.L. Brehm). Winter migrant of irregular passage and somewhat rare

Monticola saxatilis (Linnaeus, 1766)

Monticola saxatilis (L.). Summer migrant, not uncommon, it is not abundant. Breeds.

Monticola solitarius (Linnaeus, 1758)

Monticola cyanus (L.). Resident, not at all uncommon. The 'Passero solitario' or 'Solitary Sparrow', as this species is called here, is a favourite cage-bird in Sicily, and deservedly so, for it becomes in charming pet as sided-songster. When brought up from the nest, as it generally is, it is absolutely devoid of fear, and appears perfectly happy in confinement. Resident, but not very common.

Cinclus cinclus aquaticus Bechstein, 1797

Cinclus acquaticus Bechstein. Breeds in higher mountainous districts. Specimens in my collection from Madonie range.

Oenanthe oenanthe (Linnaeus, 1758)

Saxicola oenanthe (L.). Summer migrant of regular passage and most abundant. Breeds in higher dis- 


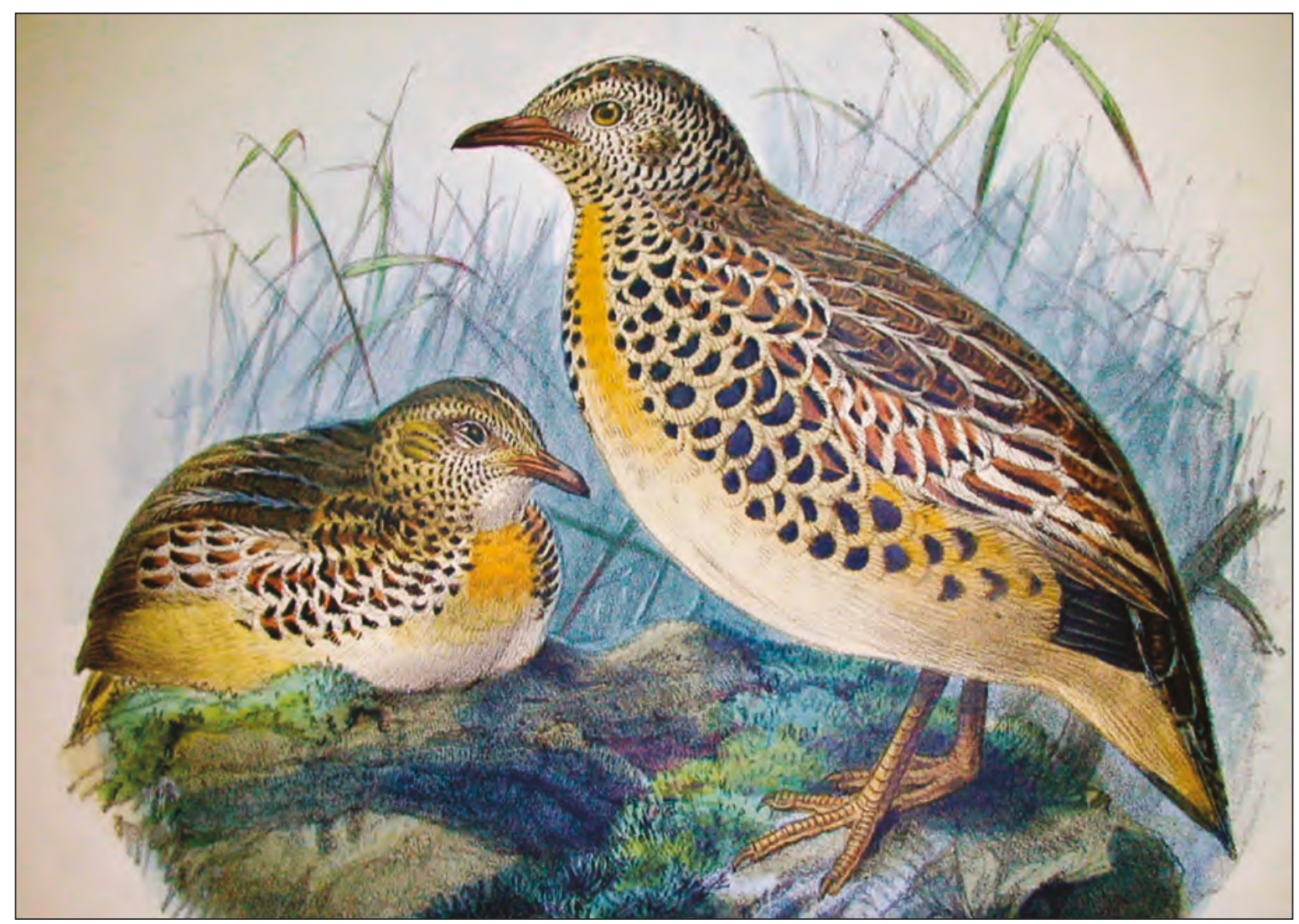

Figure 17. Common Bottonquail Turnix sylvaticus. Formerly widespread in flat areas of Sicily, dominated by the Dwarf Palm Chamaerops humilis, the last specimens found date back to ca. 1920 (after Dresser, 1871-1881).

tricts. Examples in my collection obtained from Madonie Mts. in June.

Oenanthe hispanica hispanica (Linnaeus, 1758) Saxicola melanoleuca hispanica (L.) (species names aurita and stapazina deleted). Of regular passage through, never very abundant. This, the western form, of which were originally known as the Black-throated and Black-eared Chats, now recognized as one of the same species, occurs in both of phases of plumages.

Page 2 (Fig. 19)

Oenanthe hispanica melanoleuca (Güldenstädt, 1775)

Saxicola hispanica xanthomeleuca H. \& E. Of regular passage, it is never very abundant. This, the eastern form of the originally known as the Blackthroated and Black-eared Chats occurs in both of phases of plumage.

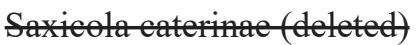

Saxiofamelanolenea (deled)

Saxicolatectis (deleted)
Oenanthe leucura (Gmelin, 1789)

Saxicola leucura $(\mathrm{Gm}$.). Said to occur in the more mountainous parts of Sicily and to breed there occasionally, but it cannot be considered as otherwise than rare. I usually have never met with it in the island.

Saxicola rubetra (Linnaeus, 1758)

Pratincola rubetra (L.). Summer migrant, common. Breeds.

Saxicola torquatus rubicola (Linnaeus, 1766)

Pratincola rubicola (L.). Resident and migratory. Breeds and winters.

Phoenicurus phoenicurus phoenicurus (Linnaeus, 1758)

Ruticilla phoenicurus (L.). Summer migrant, very common regular passage. Said to breed, also to winter in island.

Phoenicurus ochruros gibraltariensis (Gmelin, 1789)

Ruticilla tytis (Scop.). Resident and migratory. Breeds in higher wooded districts, not uncommon in winter. 
Cianecula svecica cyanecula (Meisner, 1804) Cyanecula wolfi C.L. Brehm. Irregular passage, winters, not common.

Cyanecula svecica svecica (Linnaeus, 1758)

Cyanecula suecica (L.). Is said to occur in Sicily but apparently there is no authentic record of its occurrence there.

Erithacus rubecula (Linnaeus, 1758)

Erithacus rubecula (L.). Very abundant as winter migrant, but also breeds in the more wooded and mountainous districts.

Page 3 (Fig. 20)

Luscinia megarhynchos C.L. Brehm, 1831

Daulias luscinia (L.). Summer migrant, very abundant in spring on passage. Breeds in certain numbers. Sylvia communis Latham, 1787

Sylvia rufa (Bodd.). Summer migrant, very abundant in spring on passage. Breeds in certain numbers.

Sylvia curruca (Linnaeus, 1758)

Sylvia curruca (L.). Summer migrant, not common, according to Doderlein it is abundant and breeds in some parts of the island!

Sylvia cantillans cantillans (Pallas, 1764)

Sylvia subalpina Bonelli. Summer migrant, very abundant. Breeds in certain numbers.

Sylvia conspicillata Temminck, 1820

Sylvia conspicillata Marm. Summer migrant, fairly abundant. Breeds. Specimens obtained at Mistretta end of June.

Sylvia melanocephala (Gmelin, 1789)

Sylvia melanocephala (Gm.). Resident and abundant. Sylvia hortensis (Gmelin, 1789)

Sylvia orphaea Temm. According to Doderlein this species occurs occasionally as summer migrant.

Sylvia ruppeli Temminck, 1823

Sylvia Rueppelli Temm. One example obtained at Messina, now in Florence museum.

Sylvia atricapilla (Linnaeus, 1758)

Sylvia atricapilla (L.). Resident and migratory, abundant. Breeds not only in higher woods, but also in the seabord. Ex. on our own garden at Malfitano, Palermo.

Sylvia borin (Boddaert, 1783)

Sylvia salicaria (L.). A regular migrant, more plentiful in autumn than in spring, at times very abundant only on summer season and feeds largely on figs, whence the name of 'Beccafico'.

Sylvia undata (Boddaert, 1783)

Melizophilus undatus (Bodd.). Scarce winter mi- grant, and probably also recorded in some parts of the southern seabord of Sicily, and also in the small island of Pantelleria.

Sylvia sarda Temminck, 1820

Melizophilus sardus (Marm.). Scarce winter migrant. Doderlein asserted to have found this species by no means uncommon along the south coasts of that island and specially on Pantelleria.

Regulus regulus (Linnaeus, 1758)

Regulus cristatus Koch. Winter migrant, but is said to be also resident in certain numbers. It is far less plentiful than the following species.

Regulus ignicapilla (Temminck, 1820)

Regulus ignicapillus (C.L. Brehm). Resident and migratory, abundant in winter.

Phylloscopus collybita (Vieillot, 1817)

Phylloscopus collybita (Vieill.). Of regular passage, very abundant, many in the winter months. Is said also to breed in the island.

Phylloscopus trochilus (Linnaeus, 1758)

Phylloscopus trochilus (L.). Of regular passage, very abundant, many in the winter months.

Phylloscopus sibilatrix (Bechstein, 1793)

Phylloscopus sibilatrix (Bechst.). Summer migrant, fairly abundant. Breeds.

Phylloscopus bonelli (Vieillot, 1819)

Phylloscopus bonelli (Vieill.). Summer migrant, not very common.

Page 4 (Fig. 21)

Hippolais polyglotta (Vieillot, 1817)

Hippolais polyglotta (Vieill.). Common migrant, but less often met with than the following species.

Hippolais icterina (Vieillot, 1817)

Hippolais icterina (Vieill.). Common migrant, very abundant in spring. Breeds.

Cercotrichas galactotes (Temminck, 1820)

Aedon galactodes Boie. An example obtained by Doderlein in south of the island (Avifauna, p. 340). Another preserved in Pistone's collection, Messina. Acrocephalus scirpaceus (Hermann, 1804)

Acrocephalus streperus (Vieill.). Summer migrant, breeds and perhaps winters in small numbers.

Acrocephalus palustris (Bechstein, 1898)

Acrocephalus palustris (Bechst.) Summer migrant, not often met with.

Acrocephalus arundinaceus (Linnaeus, 1758)

Acrocephalus arundinaceus (L.). Summer migrant. Breeds.

Acrocephalus paludicola (Vieillot, 1817)

Acrocephalus acquaticus (Gm.) Summer migrant. 
Acrocephalus schoenobaenus (Linnaeus, 1758) Acrocephalus schoenoboenus (L.). Summer migrant. Abundant.

Acrocephalus melanopogon (Temminck, 1823)

Lusciniola melanopogon (Temm.). Accidental.

Locustella naevia (Boddaert, 1783)

Locustella naevia (Bodd.). Of doubtful occurrence.

Locustella luscinioides (Savi, 1824)

Locustella luscinioides (Savi). Of doubtful occurrence.

Cettia cetti (Temminck, 1820)

Cettia cettii (Marm.). Resident, chiefly to be met with in marshy districts such as Syracuse, Lentini, Mazzara, etc., where it is not common. I have even obtained a specimen in January at Gibilmanna in the Madonie Mts. at an altitude of over $800 \mathrm{~m}$ !

Cisticola juncidis juncidis (Rafinesque, 1810)

Cisticola cursitans (Frankl.). Resident and migratory. Abundant.

Prunella collaris (Scopoli, 1769)

Accentor collaris (Scop.). Winter migrant, to be said to be also resident in mountainous districts, not at all common, however.

Prunella modularis (Linnaeus, 1758)

Prunella modularis (L.). Winter migrant, fairly abundant.

Page 5 (Fig. 22)

Panurus biarmicus (Linnaeus, 1758)

Panurus biarmicus (L.). According to Doderlein resident and migratory in the Pantano di Catania.

Aegithalos caudatus siculus Whitaker, 1901

Acredula sicula Whit. Resident. Varies to quarters according to season and breeds in the higher regions. The only Long-tailed Tit, I think, found in Sicily.

Parus major Linnaeus, 1758

Parus major L. Resident and probably also migratory. Abundant generally, both in higher and lower districts. Breeds on seaboard, such as on our garden at Palermo.

Periparus ater (Linnaeus, 1758)

Parus ater L. Winter migrant, not common.

Parus palustris italicus (Tschusi et Hellmayr, 1900)

Parus palustris L. Winter migrant, but possibly resident (Doderlein), not common.

Cyanistes caeruleus (Linnaeus, 1758)

Parus caeruleus L. Resident and migratory, common.

Remiz pendulinus (Linnaeus, 1758)
Aegithalus pendulinus (L.). Resident and migratory, not common.

Sitta europaea cisalpina Sachtleben, 1919

Sitta caesia Wolf. Resident, not at all uncommon in the wooded mountainous regions. Examples in my collection from Madonie obtained in June.

Certhia brachydactyla C.L. Brehm, 1820

Certhia familiaris L. Resident, not at all uncommon in the wooded mountainous regions.

Tichodroma muraria (Linnaeus, 1766)

Tichodroma muraria (L.). Said to have been obtained in Sicily on two occasions (Doderlein) but no example has been found in the recent years.

Troglodytes troglodytes (Linnaeus, 1758)

Troglodytes parvulus Koch. Resident and migratory, common.

Motacilla alba alba Linnaeus, 1758

Motacilla alba L. Winter migrant, abundant. Said to breed.

Motacilla alba yarrellii Gould, 1837

Motacilla lugubris Temm. Said to have been obtained in Sicily.

Motacilla cinerea Tunstall, 1771

Motacilla melanope Pall. Resident and migratory, not uncommon.

Motacilla flava flava Linnaeus, 1758

Motacilla flava L. Regular migrant, abundant.

Page 6 (Fig. 23)

Motacilla flava thunbergi Billberg, 1828

Motacilla viridis Gmel. According to Doderlein occurs.

Motacilla flava feldegg Michahelles, 1830

Motacilla melanocephala Liecht. According to Doderlein occurs.

Motacilla citreola citreola Pallas, 1776

Motacilla raii $\mathrm{Bp}$. Is said to occur (Giglioli).

Anthus pratensis (Linnaeus, 1758)

Anthus pratensis (L.). Winter migrant, very abundant.

Anthus cervinus (Pallas, 1811)

Anthus cervinus (Pall.) Summer migrant.

Anthus trivialis (Linnaeus, 1758)

Anthus trivialis (L.). Summer migrant, very abundant.

Anthus richardi Vieillot, 1818

Anthus richardi Vieill. Autumn (2 in my collection). Rare.

Anthus spinoletta (Linnaeus, 1758)

Anthus spinoletta (L.). Winter migrant, not common. 
Anthus campestris (Linnaeus, 1758)

Anthus campestris (L.). Regular migrant, not common.

Oriolus oriolus (Linnaeus, 1758)

Oriolus galbula L. Summer migrant, abundant. Breeds.

Lanius excubitor excubitor Linnaeus, 1758

Lanius excubitor L. Said to occur occasionally in Sicily, but I have no authentic knowledge there.

Lanius minor minor Gmelin, 1788

Lanius minor Gmel. Summer migrant, abundant in spring and breeds (Doderlein).

Lanius collurio Linnaeus, 1758

Lanius collurio L. Summer migrant, not common.

Lanius senator senator Linnaeus, 1758

Lanius auriculatus Müll. Summer migrant, very common. Breeds.

Muscicapa striata striata (Pallas, 1764)

Muscicapa grisola L. Summer migrant, very common. Breeds.

Ficedula hypoleuca (Pallas, 1764)

Muscicapa atricapilla L. Summer migrant, fairly abundant.

Ficedula albicollis (Temminck, 1815)

Muscicapa collaris Bechst. Summer migrant, fairly abundant.

Page 7 (Fig. 24)

Hirundo rustica Linnaeus, 1758

Hirundo rustica L. Regular summer migrant. Breeds.

Cecropis daurica rufula (Temminck, 1835)

Hirundo rufula Temm. Occasional common visitor.

An ex. in my collection obtained near Palermo on $13^{\text {rd }}$ april 1906.

Delichon urbicum (Linnaeus, 1758)

Chelidon urbica (L.). Summer migrant, very common. Breeds. (According to Doderlein winters in some parts?).

Riparia riparia (Linnaeus, 1758)

Cotile riparia (L.) Summer migrant, abundant.

Ptyonoprogne rupestris (Scopoli, 1769)

Cotile rupestris (Scop.). Resident and migratory, not uncommon in some localities.

Carduelis carduelis tschusii Arrigoni, 1902

Carduelis elegans Steph. Resident and abundant in most parts of the island.

Spinus spinus (Linnaeus, 1758)

Chrysomitris spinus L. Occasional winter migrant.

Serinus serinus (Linnaeus, 1766)
Serinus hortulanus Koch. Resident and abundant, is also migratory.

Chloris chloris aurantiiventris (Cabanis, 1851)

Ligurinus chloris (L.). Winter migrant, a few breed in north of island.

Coccothraustes coccothraustes (Linnaeus, 1758)

Coccothraustes vulgaris Pall. Winter migrant, abundant.

Passer italiae (Vieillot, 1817)

Passer italiae (Vieill.). Examples resembling this species have been obtained in Sicily, notably from Messina and the east-coast of the island, but so far as I am aware no absolutely typical specimens have been found.

Passer hispaniolensis (Temminck, 1820)

Passer hispaniolensis Temm. Resident and ubiquitous.

Passer montanus (Linnaeus, 1758)

Passer montanus (L.). Resident and migratory.

Petronia petronia (Linnaeus, 1766)

Passer stulta (Gmel.). Resident, fairly abundant.

Fringilla coelebs Linnaeus, 1758

Fringilla coelebs L. Winter migrant, most abundant.

Breeds in higher districts (Madonie etc.).

Fringilla montifringilla Linnaeus, 1758

Fringilla montifringilla $\mathrm{L}$. Winter migrant, somewhat rare. Several ex., however, have been obtained in this island. My collection possesses four specimens of it. Linaria cannabina mediterranea (Tschusi, 1903) Linota cannabina (L.). Winter migrant, abundant. Breeds rarely.

Page 8 (Fig. 25)

Acanthis flammea cabaret (Statius Müller, 1776) Linota rufescens (Vieill.). Of accidental occurrence. A single example obtained 14 Nov 1903 in my garden at Palermo is the first recorded capture of this species in Sicily.

Bucanetes githagineus zedlitzi (Neumann, 1907)

Erithrospiza githagenia (Licht.). Accidental. Two specimens in my collection obtained near Catania 1906. Another said to have been obtained near Girgenti [Agrigento] by Barone Caruso and a $4^{\text {th }}$ ex. is present in the Palermo museum, which was captured at Mondello near Palermo on 20 October 1891.

Pyrrhula pyrrhula europaea Vieillot, 1816

Pyrrhula europaea Vieill. Occasional winter visitor, but rare.

Loxia curvirostra (Linnaeus, 1758)

Loxia curvirostra L. Irregular visitor, occasionally 
abundant (vide my writings). Numerous specimens are present in my collection, mostly obtained during the summer and autumn months, but I have a note of the occurrence of this species also in winter (cf. "Ibis").

Emberiza melanocephala Scopoli, 1769

Emberiza melanocephala Scop. Accidental visitor that has apparently been recorded occasionally near Messina.

Emberiza calandra Linnaeus, 1758

Emberiza miliaria L. Resident and migratory, abundant.

Emberiza citrinella Linnaeus, 1758

Emberiza citrinella L. Winter migrant but uncommon (occasionally not uncommon?).

Emberiza cirlus Linnaeus, 1766

Emberiza cirlus L. Resident and migratory, fairly abundant.

Emberiza hortulana Linnaeus, 1758

Emberiza hortulana L. Resident and migratory, fairly abundant.

Emberiza cia Linnaeus, 1766

Emberiza cia L. Resident and migratory, fairly abundant. Breeds. I have specimens from Mistretta obtained end of June.

Emberiza schoeniclus Linnaeus, 1758

Emberiza schoeniclus L. Winter migrant, fairly abundant.

Galerida cristata (Linnaeus, 1758)

Galerida cristata (L.) Resident, abundant.

Alauda arvensis cantarella Bonaparte, 1850

Alauda arvensis L. Winter migrant in vast numbers. A few remain and breed in some districts. From Mistretta I have examples obtained at the end of June.

Lullula arborea pallida Sarudny, 1902

Alauda arborea L. Winter migrant but also resident to a certain extent, and breeds in higher districts.

Calandrella brachydactyla (Leisler, 1814)

Calandrella brachydactyla (Leisl.). Summer visitor, abundant, in some districts breeds. Examples obtained in June with nest and eggs from San Pantaleo [Stagnone Is., Marsala].

Melanocorypha calandra (Linnaeus, 1766)

Melanocorypha calandra (L.). Resident, abundant. A favourite cage-bird in Sicily.

Page 9 (Fig. 26)

Sturnus vulgaris Linnaeus, 1758

Sturnus vulgaris $\mathrm{L}$. Winter migrant in vast numbers.
Sturnus unicolor Temminck, 1820

Sturnus unicolor De la Marm. Resident and fairly abundant in higher mountains. Exp. in my collection from Madonie obtained in June.

Pastor roseus (Linnaeus, 1758)

Pastor roseus (L.). Of occasional but rare occurrence.

Pyrrhocorax pyrrhocorax erythroramphos (Vieillot, 1817)

Pyrrhocorax graculus (L.). Resident and abundant in some parts.

Nucifraga caryocatactes (Linnaeus, 1758)

Nucifraga caryocatactes (L.). Said to have occurred. One specimen from Malta in my collection. Garrulus glandarius albipectus Kleinschmidt, 1820 Garrulus glandarius (L.) Resident and fairly abundant.

Pica pica (Linnaeus, 1758)

Pica rustica (Scop.). Resident and common.

Corvus monedula spermologus Vieillot, 1817

Corvus monedula L. Resident and in some parts abundant, both in the mountainous districts of the interior as well as on the coast.

Corvus corone corone Linnaeus, 1758

Corvus corone L. Winter migrant but not common, and occasionally to be met with in considerable numbers.

Corvus corone cornix Linnaeus, 1758

Corvus cornix L. Resident and generally distributed.

Corvus frugilegus Linnaeus, 1758

Corvus frugilegus $\mathrm{L}$. Winter migrant, at times fairly abundant.

Corvus corax corax Linnaeus, 1758

Corvus corax L. Resident and of general distribution.

Apus apus (Linnaeus, 1758)

Cypselus apus (L.). Summer migrant, common. Breeds in some towns in considerable numbers.

Tachymarptis melba (Linnaeus, 1758)

Cypselus melba (L.). Summer migrant, common. Breeds in some mountains. Appears at times in March, it is generally a late visitor.

Caprimulgus europaeus meridionalis Hartert, 1896 Caprimulgus europaeus L. Summer migrant, very plentiful. Breeds occasionally.

Caprimulgus aegyptius saharae Erlanger, 1899 Caprimulgus aegyptius Licht. Accidental. A specimen $q$ obtained 23.IV.1899, near Palermo, preserved in my collection. 
Caprimulgus ruficollis desertorum Erlanger, 1899 Caprimulgus ruficollis Temm. Accidental. A specimen $q$ obtained 3 June 1898 near Trapani, preserved in Palermo museum.

Page 10 (Fig. 27)

Dryocopus martius (Linnaeus, 1758)

Dryocopus martius (L.). Said to have been housed in mountainous forests of the interior.

Dendrocopos major pinetorum (C.L. Brehm, 1831) Picus major L. Resident, but not abundant anywhere. Leiopicus medius (Linnaeus, 1758)

Picus medius L. Said to have been obtained occasionally.

Dryobates minor buturlini Hartert, 1912

Picus minor L. It has been met with occasionally and is probably resident, but rare.

Picus viridis Linnaeus, 1758

Gecinus viridis (L.). Resident, but not common.

Jynx torquilla tschusii Kleinschmidt, 1907

Yunx torquilla L. Summer migrant, it is at times also to be met with in winter, abundant, perhaps breeds.

Alcedo atthis Linnaeus, 1758

Alcedo ispida L. Resident and generally distributed, it is moderately abundant.

Coracias garrulus Linnaeus, 1758

Coracias garrula L. Summer migrant, abundant and breeds.

Merops apiaster Linnaeus, 1758

Merops apiaster L. Summer migrant, most abundant, a few breed in the island.

Merops persicus Pallas, 1773

Merops persicus Pall. It has been obtained occasionally. A specimen was obtained near Marsala in May 1912 and was preserved in the "Scuola Magistrale" of that town. Another ex. existed on the collection of Prof. Pistone, and I presume, probably, lost in the great-earthquake of 1908.

Upupa epops Linnaeus, 1758

Upupa epops L. Summer migrant, most abundant. Breeds.

Cuculus canorus canorus Linnaeus, 1758

Cuculus canorus L. Summer migrant, most abundant. Breeds. A nest of P. rubicola in my coll. with a cuckoos' egg on it obtained in June in Madonie.

Clamator glandarius (Linnaeus, 1758)

Coccystes glandarius (L.) Accidental, but several ex. are said to have been obtained in the beach of this island. Pistone's collection of Messina contained one.
Tyto alba (Scopoli, 1769)

Strix flammea L. Resident and common.

Asio otus (Linnaeus, 1758)

Asio otus (L.). Resident and apparently not uncommon in mountainous districts.

Asio flammeus (Pontoppidan, 1763)

Asio accipitrinus (Pall.). Summer migrant, abundant. Said to be also resident. I have shot an ex. myself on $6^{\text {th }}$ February.

Strix aluco sylvatica Shaw, 1809

Syrnium aluco (L.). Resident, not uncommon.

Otus scops (Linnaeus, 1758)

Scops giu (Scop.). Summer migrant, common. Breeds.

Bubo bubo (Linnaeus, 1758)

Bubo bubo (L.). Resident, but not common.

Athene noctua (Scopoli, 1769)

Athene noctua (Retz.). Resident and not uncommon. Received its eggs from Ficuzza.

Page 11 (Fig. 28)

Gyps fulvus (Hablizl, 1783)

Gyps fulvus (Gmel.). Resident and by no means uncommon.

Aegypius monachus (Linnaeus, 1766)

Vultur monachus $\mathrm{L}$. Said to have formerly occurred, but no instance of its occurrence in the island has been recorded for a very long time.

Neophron percnopterus (Linnaeus, 1758)

Neophron percnopterus (L.). Summer migrant and probably also resident to a certain extent. Breeds in island.

Gypaetus barbatus (Linnaeus, 1758)

Gypaetus barbatus (L.) Used formerly to be resident and apparently not very uncommon in the higher mountains of Sicily, but no ex. of it have been obtained for many years.

Circus aeruginosus (Linnaeus, 1758)

Circus aeruginosus (L.). Resident and fairly abundant.

Circus pygargus (Linnaeus, 1758)

Circus cineraceus (Nord.). Regular migrant, not uncommon.

Circus cyaneus (Linnaeus, 1766)

Circus cyaneus (L.). Resident and fairly abundant.

Circus macrourus (Gmelin, 1770)

Circus swainsoni Smith. Regular migrant, not uncommon.

Buteo buteo buteo (Linnaeus, 1758)

Buteo vulgaris Leach. Resident, fairly abundant. 
Clanga pomarina (C.L. Brehm, 1831)

Aquila pomarina C.L. Brehm. Resident in the interior of the island. Rare.

Aquila chrysaetos (Linnaeus, 1758)

Aquila chrysaetus (L.). Resident, but more or less rare now. I have received 2 specimens and eggs from Castelbuono and of two individuals kept one in confinement nearly 7 years.

Aquila fasciata Vieillot, 1822

Nisaetus fasciatus (Vieill.). Resident, but more or less rare now.

Haliaeetus albicilla (Linnaeus, 1758)

Haliaetus albicilla (L.) Occasionally migrant, but rarely.

Circaetus gallicus (Gmelin, 1788)

Circaetus gallicus (Gmel.). Summer migrant, not uncommon.

Accipiter gentilis Linnaeus, 1758

Astur palumbarius (L.). Resident, but not common. Accipiter nisus (Linnaeus, 1758)

Accipiter nisus (L.). Resident and migratory, not uncommon.

Milvus milvus (Linnaeus, 1758)

Milvus ictinus Savig. Resident and migratory, not uncommon.

Milvus migrans (Boddaert, 1783)

Milvus migrans (Bodd.). Summer migrant, rare.

Page 12 (Fig. 29)

Pernis apivorus (Linnaeus, 1758)

Pernis apivorus (L.). Regular migrant, most abundant in spring.

Falco peregrinus brookei Sharpe, 1873

Falco peregrinus Tunstall. Resident and migratory, not uncommon.

Falco peregrinus minor Bonaparte, 1850

Falco minor Bp. Of occasional occurrence [It is considered synonymous with $F$. peregrinus brookei].

Falco peregrinus pelegrinoides Temminck, 1829

Falco barbarus L. [No data reported]

Falco biarmicus feldeggii Schlegel, 1843

Falco feldeggi Schlegel. Accidental.

Falco cherrug Gray, 1834

Falco sacer Gmel. Accidental. An ex. preserved in the Bordonaro's collection, which was obtained at the Villa Camastra [presently Villa Tasca], Palermo.

Falco subbuteo Linnaeus, 1758

Falco subbuteo L. Regular migrant. Breeds.

Falco columbarius aesalon Tunstall, 1771

Falco aesalon Tunstall. Irregular migrant.
Falco vespertinus Linnaeus, 1766

Falco vespertinus $\mathrm{L}$. Regular migrant, abundant.

Falco eleonorae Géné, 1839

Falco eleonorae Gené. Of occasional occurrence.

Falco tinnunculus Linnaeus, 1758

Falco tinnunculus L. Resident and abundant.

Falco naumanni Fleischer, 1818

Falco cenchris Naum. Resident and abundant.

Pandion haliaetus (Linnaeus, 1758)

Pandion haliaetus (L.). Winter migrant and not uncommon. Perhaps resident also.

Phalacrocorax carbo sinensis (Staunton, 1796)

Phalacrocorax carbo (L.). Resident and abundant.

Gulosus aristotelis desmarestii (Payraudeau, 1826)

Phalacrocorax graculus (L.). Resident and abundant.

Microcarbo pygmaeus (Pallas, 1773)

Phalacrocorax pygmaeus Pall. Of accidental occurrence. An ex. in the collection of the avv.to Mauceri,

Catania.

Morus bassanus (Linnaeus, 1758)

Sula bassana (L.). Of occasional occurrence, it is probably not as uncommon as is generally supposed. My collection has a fine $\delta$ ad. specimen, and also an immature specimen, both captured near Palermo.

Pelecanus onocrotalus Linnaeus, 1758

Pelecanus onocrotalus L. Of occasional occurrence. An ex. is preserved in the collection of the "Liceo" at Trapani.

Pelecanus crispus Bruch, 1832

Pelecanus crispus Bruch. An example, a $\hat{O}$, of this species preserved in the Palermo Museum was captured near Camporeale in 1890.

Page 13 (Fig. 30)

Ardea cinerea Linnaeus, 1758

Ardea cinerea L. Of regular passage, winters, said to breed.

Ardea purpurea Linnaeus, 1766

Ardea purpurea L. Of regular passage, winters, said to breed.

Ardea alba Linnaeus, 1758

Ardea alba $\mathrm{L}$. Said to be of regular passage and in some years not uncommon.

Egretta garzetta (Linnaeus, 1766)

Ardea garzetta L. Of regular passage, more abundant in spring.

Ardeola ralloides (Scopoli, 1769)

Ardea ralloides Scop. Spring migrant, abundant in some years. 
Ixobrychus minutus (Linnaeus, 1766)

Ardetta minuta (Linn.). Spring migrant, abundant in some years. Breeds.

Nycticorax nycticorax (Linnaeus, 1758)

Nycticorax griseus (L.). Of regular passage, at times very abundant.

Botaurus stellaris (Linnaeus, 1758)

Bottaurus stellaris (L.). Resident and migratory, not uncommon.

Ciconia ciconia Linnaeus, 1758

Ciconia alba Bechst. Of occasional occurrence on passage. Not uncommon in some years.

Ciconia nigra Linnaeus, 1758

Ciconia nigra (L.). Of occasional occurrence on passage. Not uncommon in some years.

Platalea leucorodia Linnaeus, 1758

Platalea leucorodia L. Regular migrant and winters.

Plegadis falcinellus (Linnaeus, 1766)

Plegadis falcinellus (L.). Regular migrant, at times abundant in spring.

Phoenicopterus roseus Pallas, 1811

Phoenicopterus roseus Pall. Of occasional occurrence on passage.

Anser anser (Linnaeus, 1758)

Anser cinereus Meyer. Of occasional occurrence in winter, rare.

Anser fabalis (Latham, 1787)

Anser segetum (Gmel.). Of occasional occurrence in winter, rare.

Anser albifrons (Scopoli, 1769)

Anser albifrons (Scop.). Of occasional occurrence in winter, rare.

Branta bernicla (Linnaeus, 1758)

Bernicla branta (Pall.). Winter migrant, at times not uncommon.

Cygnus cygnus (Linnaeus, 1758)

Cygnus musicus Bechst. Of occasional occurrence in winter, rare.

Tadorna tadorna (Linnaeus, 1758)

Tadorna cornuta (Gmel.). Winter migrant, not uncommon.

Tadorna ferruginea (Pallas, 1764)

Tadorna casarca (L.). Of accidental occurrence.

Page 14 (Fig. 31)

Anas platyrhynchos Linnaeus, 1758

Anas boscas L. Winter migrant, abundant, some breed.

Marmaronetta angustirostris Ménétries, 1832

Anas angustirostris Ménétr. Of irregular occurrence, but in some years not uncommon in spring.

Mareca strepera (Linnaeus, 1758)

Chaulelasmus strepera (L.). Winter migrant, not uncommon.
Spatula clypeata (Linnaeus, 1758)

Spatula clypeata (L.). Winter migrant, not uncommon. Anas crecca Linnaeus, 1758

Anas crecca (L.). Winter migrant, very abundant. Breeds (Doderlein).

Spatula querquedula (Linnaeus, 1758)

Querquedula circia L. Summer migrant in vast flocks.

Anas acuta Linnaeus, 1758

Dafila acuta (L.). Winter migrant, abundant.

Mareca penelope (Linnaeus, 1758)

Mareca penelope (L.). Winter migrant, abundant.

Aythya ferina (Linnaeus, 1758)

Fuligula ferina (L.). Winter migrant, abundant.

Netta rufina (Pallas, 1773)

Fuligula rufina (Pall.). Winter migrant, not uncommon and breeds (Doderlein).

Aythya marila (Linnaeus, 1761)

Fuligula marila (L.). [No data reported].

Aythya fuligula (Linnaeus, 1758)

Fuligula cristata (Leach). Winter migrant, not uncommon.

Aythya nyroca (Güldenstädt, 1770)

Nyroca ferruginea (Gmel.). Winter migrant, abundant, perhaps breeds.

Bucephala clangula (Linnaeus, 1758)

Clangula glaucion (L.). Winter migrant, but rather rare.

Melanitta fusca (Linnaeus, 1758)

Oedemia fusca (L.). Of occasional occurrence. Has been met with near Siracusa (Doderlein).

Oxyura leucocephala (Scopoli, 1769)

Erismatura leucocephala (Scop.). Winter migrant, not abundant.

Mergus serrator Linnaeus, 1758

Mergus serrator L. Winter migrant, abundant.

Mergellus albellus (Linnaeus, 1758)

Mergus albellus L. Occasionally met with in winter.

Columba palumbus Linnaeus, 1758

Columba palumbus L. Of regular passage.

Columba livia Gmelin, 1789

Columba livia Bonnat. Resident and abundant.

Columba oenas Linnaeus, 1758

Columba oenas L. Of regular passage.

Streptopelia turtur (Linnaeus, 1758)

Turtur communis Selby. Summer migrant, very abundant and breeds. Occasionally has been met with even in winter. During the winters 1911, 1912 and 1913 observed near Randello [province of Ragusa] (N. Arezzo). 
Pterocles senegallus (Linnaeus, 1771)

Pterocles senegallus L. My collection possesses the only ex. of this species so far recorded not only from Italy, but also from the whole of Europe! The specimen in question is a $q$ and was obtained on 28 April 1910 on the Arezzo's estate of Randello near Santa [Croce di] Camarina in the province of Syracuse. It was sent to me in the flesh, by my friend Duca di Celano, son of the Marchese Arezzo, also an old friend of my youth. The bird, when shot was in company of another of the same species, probably the male (vide "Ibis" and "Avicula").

Page 15 (Fig. 32)

Alectoris graeca whitakeri Schiebel, 1934

Caccabis saxatilis (Meyer). Resident and generally distributed throughout the more hilly districts of the island. Albino exp. not uncommon.

Francolinus francolinus asiae Bonaparte, 1856 Francolinus vulgaris Steph. Up to about 50 years ago a resident species unfairly, but now extinct (cf... Probably he referred to the paper of Lilford, 1862).

Coturnix coturnix (Linnaeus, 1758)

Coturnix communis Bonnat. Resident and migratory in vast numbers, it is less abundant of late years, owing no doubts its netting. Breeds in considerable quantity and a good many also winter.

Turnix sylvaticus sylvaticus (Desfontaines, 1789) Turnix sylvatica (Desf.). Up to some 40 years ago a resident and not uncommon species. It appears, however, now to be extinct, for no occurrence of it has been recorded for several years.

Rallus aquaticus Linnaeus, 1758

Rallus aquaticus L. Resident and migratory.

Porzana porzana (Linnaeus, 1758)

Porzana maruetta (Leach.). Summer migrant, a few apparently breed and a few also winter in the island.

Zapornia pusilla intermedia (Hermann, 1804)

Porzana bailloni (Vieill.). Of regular passage, but also winters.

Zapornia parva (Scopoli, 1769)

Porzana parva (Scop.). Of regular passage, but also winters.

Crex crex (Linnaeus, 1758)

Crex pratensis Bechst. Summer migrant, a few apparently breed and winter in the island.

Porphyrio porphyrio porphyrio (Linnaeus, 1758) Porphyrio caeruleus (Vandelli). Resident and possibly also migratory to a certain extent. Not uncom- mon, it is threatened with extinction, owing to the general reclaiming of marshy lands.

Porphyrio porphyrio madagascariensis (Latham, 1801)

Porphyrio smaragdinus Temm. Said to have occurred occasionally in the island. Exs. of this species reporting to come from Sicily are preserved in the Florence and Naples museums.

Porphyrio alleni Thomson, 1842

Porphyrio alleni Thomson. Said to have occurred occasionally in the island. An ex. in my collection obtained in $4^{\text {th }}$ December 1902 at the Pantano di Catania is the $5^{\text {th }}$ recorded occurrence of this species for Europe. Another individual seen in the same winter at Catania. An ex. from Tunis/or rather Bizerte sent me in the same winter.

Gallinula chloropus (Linnaeus, 1758)

Gallinula chloropus (L.). Resident and migratory, abundant.

Fulica cristata Gmelin, 1789

Fulica cristata Gmel. Of accidental and rare occurrence (Doderlein).

Fulica atra Linnaeus, 1758

Fulica atra L. Winter migrant, very abundant.

Grus grus grus (Linnaeus, 1758)

Grus communis Bechst. Of regular passage in large flocks.

Anthropoides virgo (Linnaeus, 1758)

Grus virgo (L.). Of accidental and rare occurrence.

Otis tarda Linnaeus, 1758

Otis tarda L. Of accidental and rare occurrence. An ex. of this species purportedly to have been obtained in this island is present in the Lyceum Museum of Trapani.

Tetrax tetrax (Linnaeus, 1758)

Otis tetrax L. Resident and once not uncommon in the southern districts of Sicily. In recent years appears to be much rarer.

Burhinus oedicnemus oedicnemus (Linnaeus, 1758) Oedicnemus scolopax (Gmel.). Of regular passage and possibly resident to a certain extent.

Glareola pratincola (Linnaeus, 1766)

Glareola pratincola L. Summer migrant, not uncommon, is said to breed.

Glareola nordmanni Fischer von Waldheim, 1842 Glareola melanoptera Nordm. Of very rare occurrence. An ex., $\hat{\partial}$, preserved in my collection was obtained towards the end of April 1904 near Marsala. This is the only recorded mention of its occurrence in Sicily and the $2^{\text {nd }}$ of the whole of 
Italy. Count Arrigoni mentions a capture in 1892 at Bagnolo near Vicenza (R[ivista] iT[aliana] Orn[itologia] ii. p. 362).

Page 16 (Fig. 33)

Cursorius cursor (Latham, 1787)

Cursorius gallicus (Gmel.). Of occasional occurrence in spring. About half of dozen ex. of this species appear to have been obtained at various times.

Pluvialis apricaria apricaria (Linnaeus, 1758)

Charadrius pluvialis L. Winter migrant, very abundant from November to March.

Pluvialis squatarola (Linnaeus, 1758)

Squatarola helvetica (L.). Of regular passage, not uncommon.

Charadrius alexandrinus Linnaeus, 1758

Aegialites cantiana (Galli). Of regular passage, not uncommon. Breeds.

Charadrius dubius curonicus Gmelin, 1789

Aegialites curonica (Gmel.). Of regular passage, not uncommon.

Charadrius hiaticula Linnaeus, 1758

Aegialites hiaticula (L.). Of regular passage, not uncommon. Winters.

Eudromias morinellus (Linnaeus, 1758)

Eudromias morinellus (L.). Of regular passage, not uncommon. Winters.

Vanellus vanellus (Linnaeus, 1758)

Vanellus vulgaris Bechst. Winter migrant, abundant.

Arenaria interpres (Linnaeus, 1758)

Strepsilas interpres (L.). Of irregular passage.

Haematopus ostralegus Linnaeus, 1758

Haematopus ostralegus L. Of irregular passage.

Recurvirostra avosetta Linnaeus, 1758

Recurvirostra avosetta L. Of irregular passage.

Himantopus himantopus (Linnaeus, 1758)

Himantopus candidus Bonnat. Of regular passage.

Phalaropus fulicarius (Linnaeus, 1758)

Phalaropus fulicarius L. Of occasional occurrence.

Scolopax rusticola (Linnaeus, 1758)

Scolopax rusticola L. Winter migrant, abundant.

Gallinago media (Latham, 1787)

Gallinago major (Gmel.) Of regular passage, but not common.

Gallinago gallinago (Linnaeus, 1758)

Gallinago caelestis (Frenzol). Of regular passage, abundant in winter.

Lymnocryptes minimus (Brünnich, 1764)

Gallinago gallinula L. Of regular passage, abundant in winter.
Calidris falcinellus (Pontoppidan, 1763)

Limicola platyrhynca Temm. Of accidental occurrence.

Calidris alpina (Linnaeus, 1758)

Tringa alpina L. Winters irregularly, very abundant in spring.

Calidris minuta (Leisler, 1812)

Tringa minuta Leisl. Winters irregularly, very abundant in spring.

Calidris ferruginea (Pontoppidan, 1763)

Tringa subarquata (Güld.) Winters irregularly, very abundant in spring.

Calidris canutus (Linnaeus, 1758)

Tring a canuta L. Of rare occurrence.

Calidris pugnax (Linnaeus, 1758)

Machetes pugnax (L.). Of regular passage, abundant.

Calidris alba (Pallas, 1764)

Calidris arenaria (L.). Of regular passage, abundant. Actitis hypoleucos (Linnaeus, 1758)

Totanus hypoleucus (L.). Of regular passage, abundant, possibly resident in certain extent.

Tringa ochropus Linnaeus, 1758

Totanus ochropus (L.). Of regular passage, abundant, possibly resident in certain extent.

Page 17 (Fig. 34)

Tringa glareola Linnaeus, 1758

Totanus glareola (L.). Of regular passage, abundant in some localities.

Tringa stagnatilis (Bechstein, 1803)

Totanus stagnatilis Bechst. Of regular passage, not very common.

Tringa totanus (Linnaeus, 1758)

Totanus calidris (L.). Of regular passage, winters.

Tringa erythropus (Pallas, 1764)

Totanus fuscus (L.). Of regular passage, fairly abundant, winters.

Tringa nebularia (Gunnerus, 1767)

Totanus canescens (Gmel.). Of regular passage, not common.

Limosa lapponica (Linnaeus, 1758)

Limosa lapponica (L.). Said to have occurred rarely (Doderlein).

Limosa limosa (Linnaeus, 1758)

Limosa aegocephala (L.) (limosa). Of regular passage, but not very common.

Numenius phaeopus (Linnaeus, 1758)

Numenius phaeopus (L.). Winter migrant, not un common. 
Numenius tenuirostris Vieillot, 1817

Numenius tenuirostris Vieill. Of regular passage, winters, common.

Numenius arquata (Linnaeus, 1758)

Numenius arquata (L.). Winter migrant, abundant.

Sterna hirundo Linnaeus, 1758

Sterna fluviatilis Naum. Summer migrant, abundant, said to breed (Doderlein).

Sternula albifrons (Pallas, 1764)

Sterna minuta L. Summer migrant, abundant. Breeds.

Thalasseus bengalensis emigratus (Neumann, 1934) Sterna media Cretz. Of accidental occurrence and very rare. It has twice been obtained in Sicily (Doderlein).

Hydroprogne caspia (Pallas, 1770)

Sterna caspia Pall. Occasionally met with, but rarely.

Gelochelidon nilotica (Gmelin, 1789)

Sterna anglica Mont. Regular migrant and not uncommon at times.

Sterna sandvicensis (Latham, 1787)

Sterna cantiaca Gmel. Regular migrant, abundant in spring and probably breeds.

Chlidonias hybrida (Pallas, 1811)

Hydrochelidon hybrida (Pall.). Of occasional occurrence but rare.

Chlidonias leucopterus (Temminck, 1815)

Hydrochelidon leucoptera (Schinz). Of regular passage, abundant.

Chlidonias niger (Linnaeus, 1758)

Hydrochelidon nigra (L.). Summer migrant, abundant and said to breed.

Larus ridibundus Linnaeus, 1766

Larus ridibundus L. Of regular passage, abundant, probably resident.

Larus melanocephalus Temminck, 1820

Larus melanocephalus Temm. Of regular passage, abundant, probably resident.

Hydrocoloeus minutus (Pallas, 1776)

Larus minutus Pall. Of irregular passage, abundant in some winters.

Larus canus Linnaeus, 1758

Larus canus L. A winter migrant, not uncommon.

Larus genei Brème, 1839

Larus gelastes Licht. Winter migrant, rare.

Larus audouinii Payraudeau, 1826

Larus audouini Payr. Resident, it is no so here abundant or at all generally distributed.
Larus michahellis Naumann, 1840

Larus cachinnans Pall. Resident and common.

Larus fuscus Linnaeus, 1758

Larus fuscus L. Resident and common.

Larus marinus Linnaeus, 1758

Larus marinus L. Of very rare occurrence.

Rissa tridactyla (Linnaeus, 1758)

Rissa tridactyla (L.). Of irregular passage, it is abundant at times and winters.

Stercorarius (Temminck, 1815)

Stercorarius pomatohrinus (Temm.). Of rare occurrence in winter.

Page 18 (Fig. 35)

Hydrobates pelagicus melitensis (Schembri, 1843)

Procellaria pelagica L. Resident and not uncommon.

Calonectris diomedea (Scopoli, 1769)

Puffinus kuhli (Boie). Resident and abundant (Fig. 32).

Puffinus yelkouan (Acerbi, 1827)

Puffinus anglorum yelkouan. Resident and not uncommon

Alca torda Linnaeus, 1758

Alca torda L. Of irregular occurrence in winter. In some years not uncommon.

Fratercula arctica (Linnaeus, 1758)

Fratercula arctica (L.). Of irregular occurrence in winter. In some years not uncommon.

Gavia arctica (Linnaeus, 1758)

Colymbus arcticus. An occasional winter visitor. An ex. in my collection obtained in the winter of 1904 on the Lake of Lentini.

Gavia stellata (Pontoppidan, 1763)

Colymbus septentrionalis L. Of occasional appearance in winter, an ex. in my collection obtained in 1904.

Podiceps cristatus (Linnaeus, 1758)

Podiceps cristatus (L.). Resident and migratory, common in winter.

Podiceps auritus (Linnaeus, 1758)

Podiceps auritus (L.). Of occasional, but rare occurrence in winter

Podiceps nigricollis (C.L. Brehm, 1831)

Podiceps nigricollis (C.L. Brehm). Resident and migratory, not uncommon (Fig. 32).

Tachybaptus ruficollis (Pallas, 1764)

Podiceps fluviatilis (Tunstall). Resident and migratory, not uncommon. 


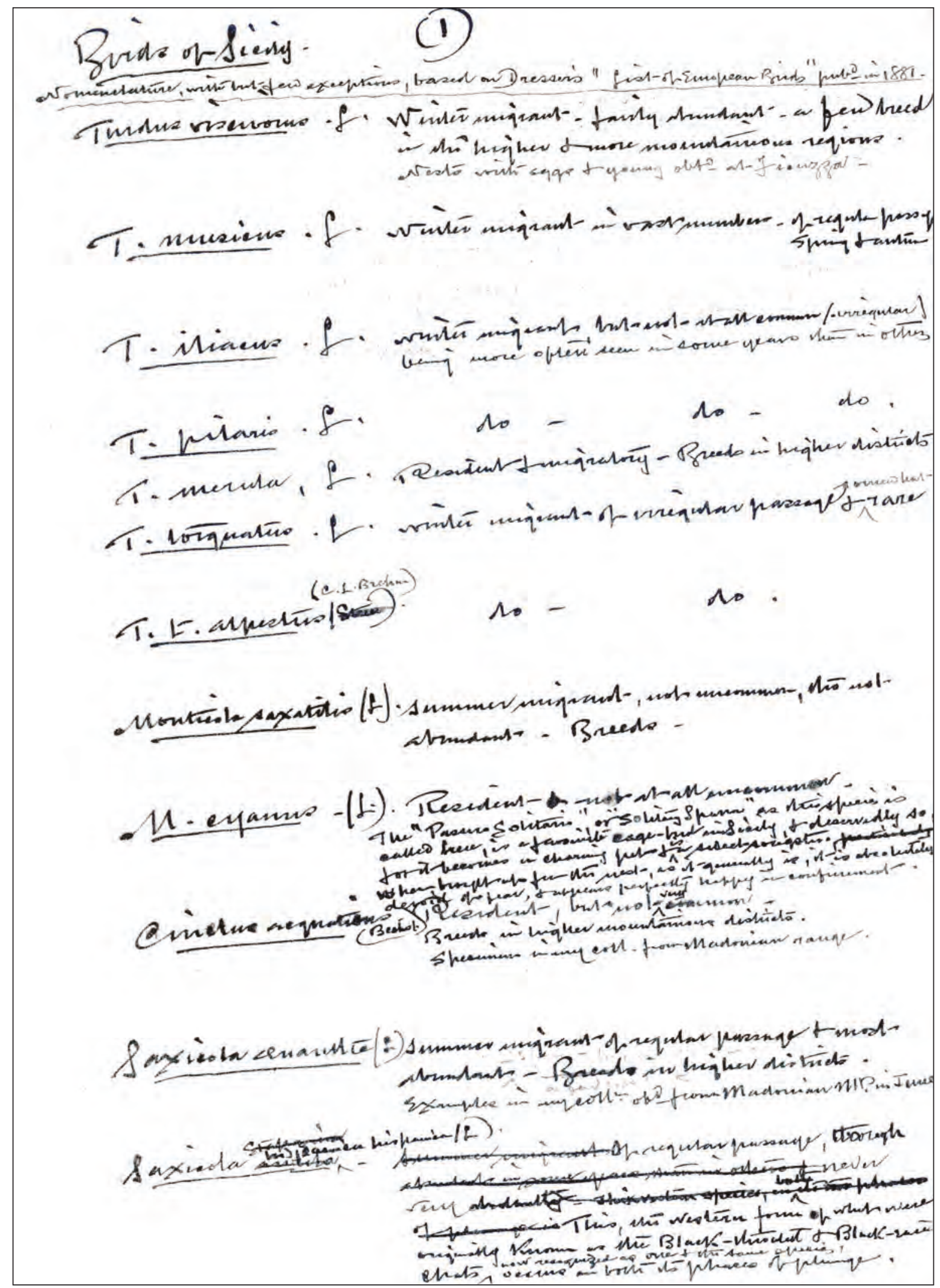

Figure 18. Page 1 of Birds of Sicily by Joseph Whitaker (ca. 1920). 


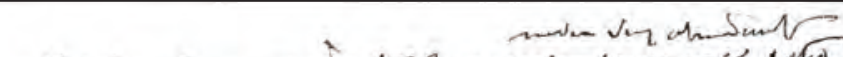

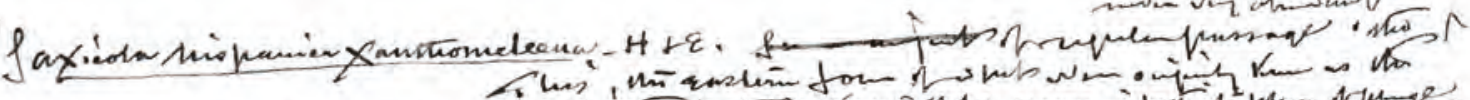

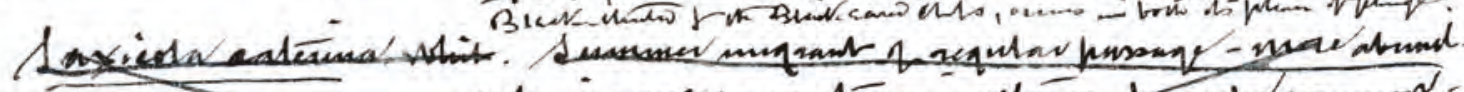

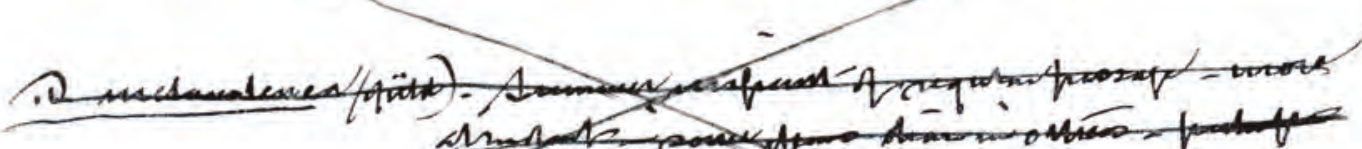

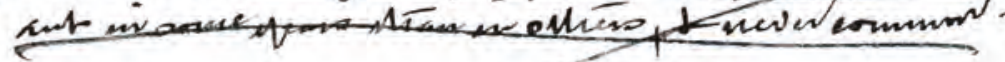

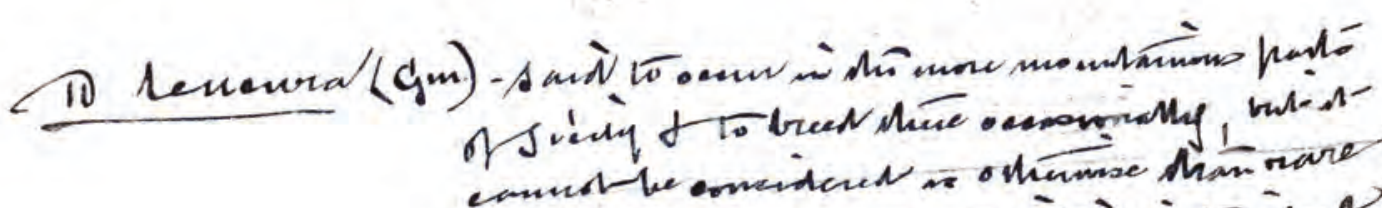

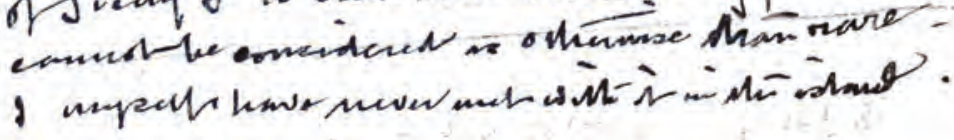

Pratimedspuletiñ (f)

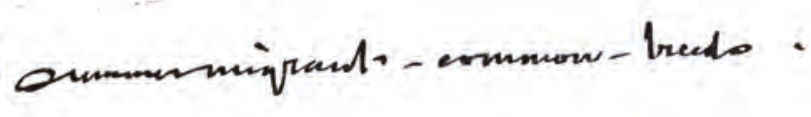

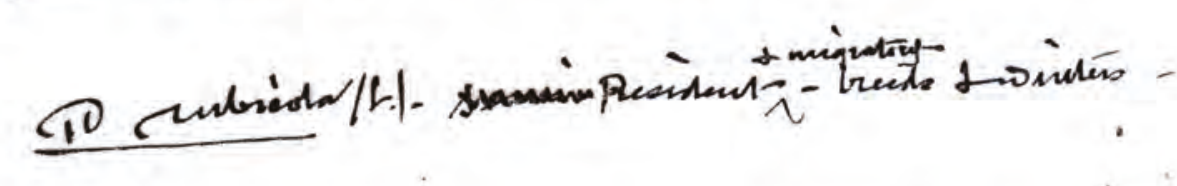

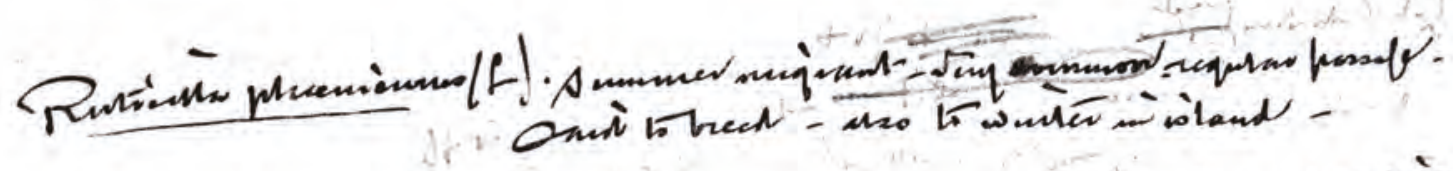

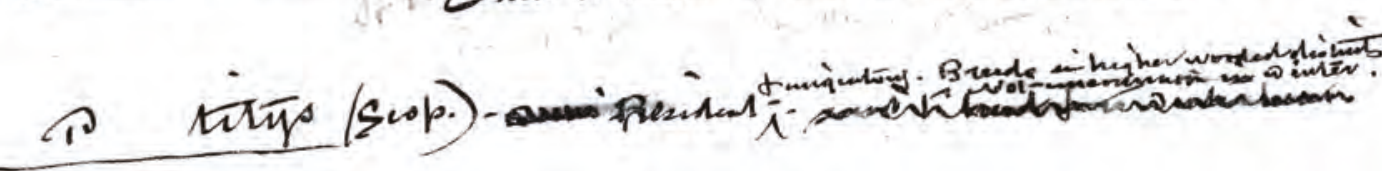

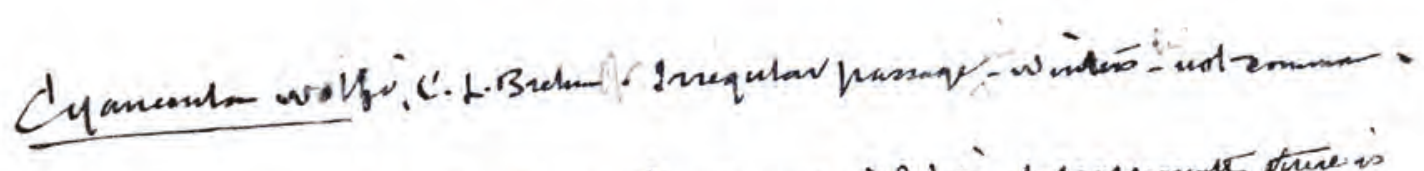

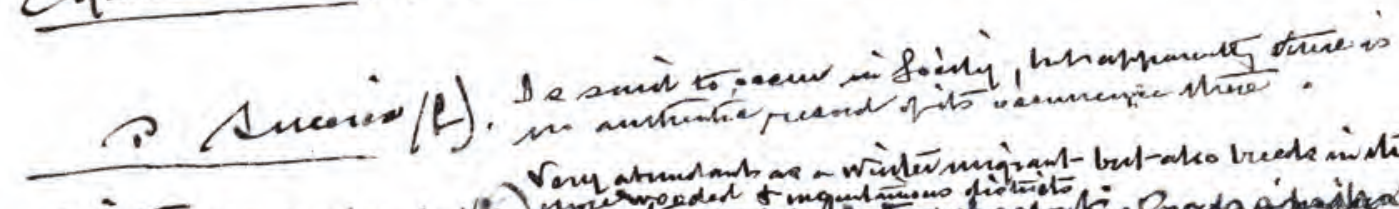

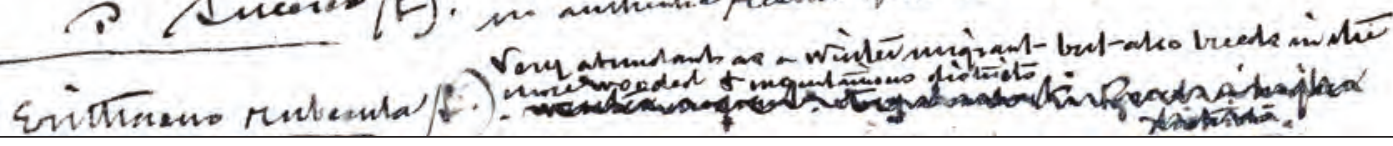

Figure 19. Page 2 of Birds of Sicily by Joseph Whitaker (ca. 1920). 


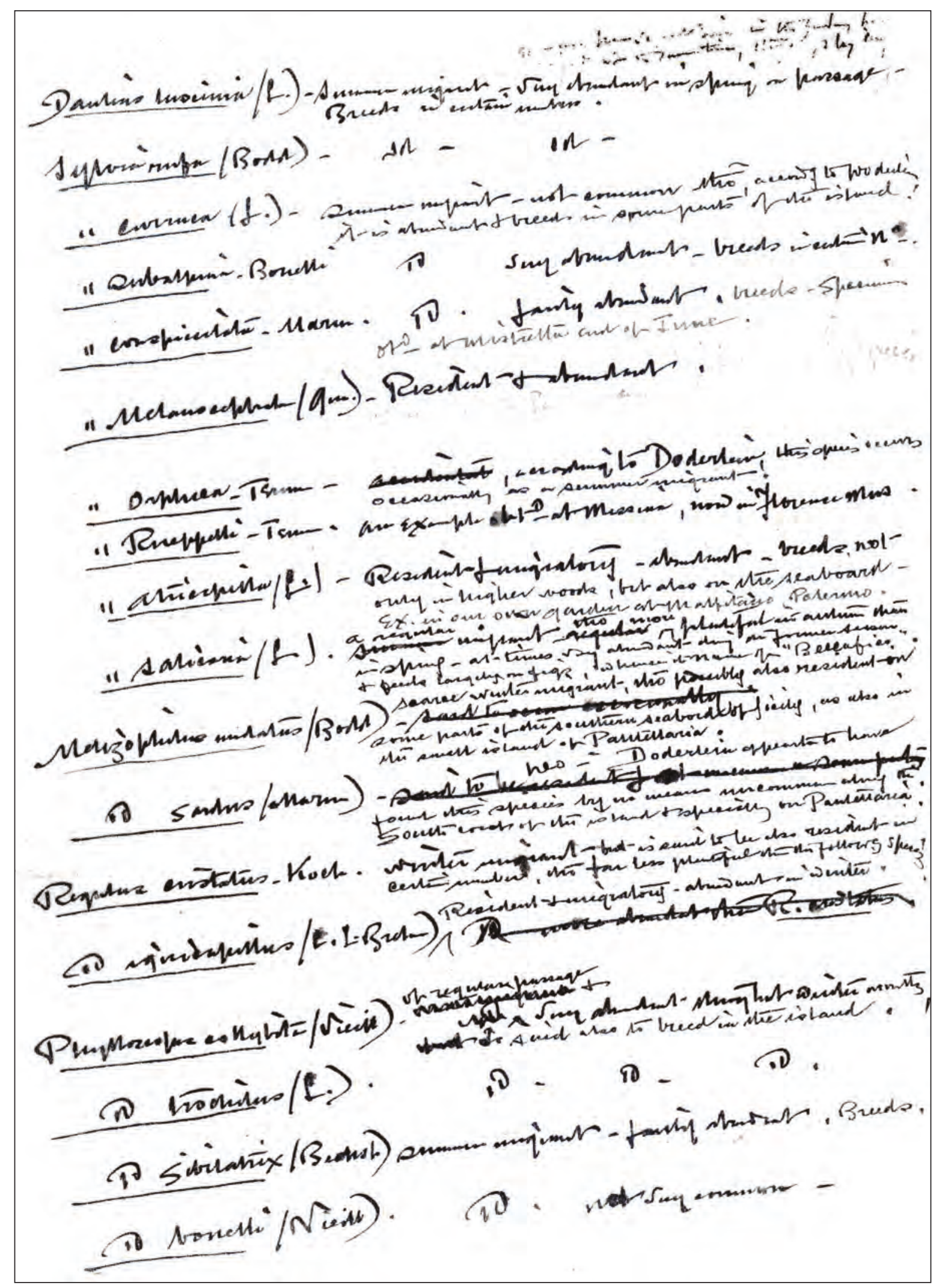

Figure 20. Page 3 of Birds of Sicily by Joseph Whitaker (ca. 1920). 
One hundred and fifty years of ornithology in Sicily, with an unknown manuscript by Joseph Whitaker

71

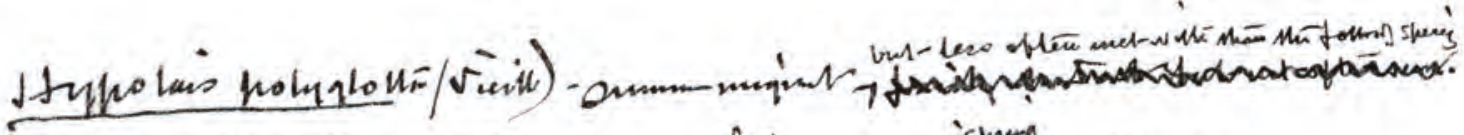

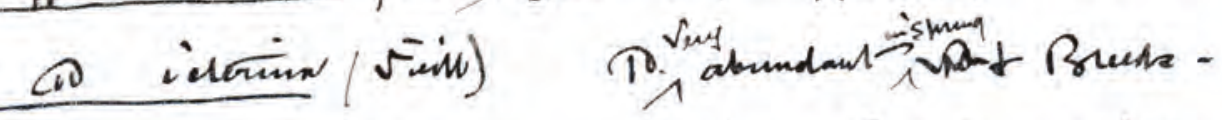

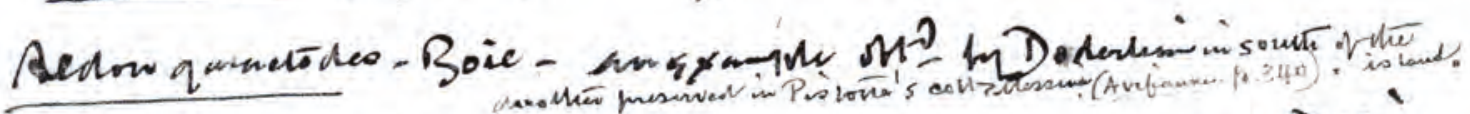

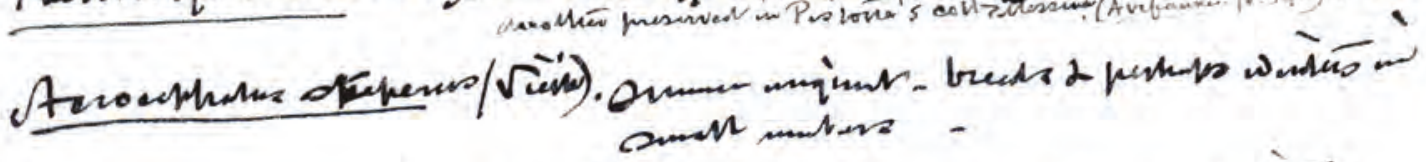

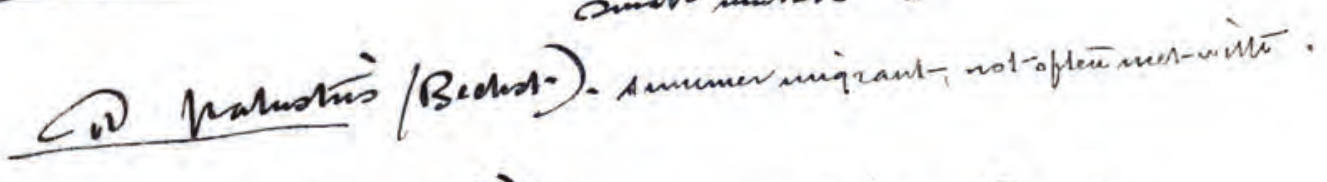

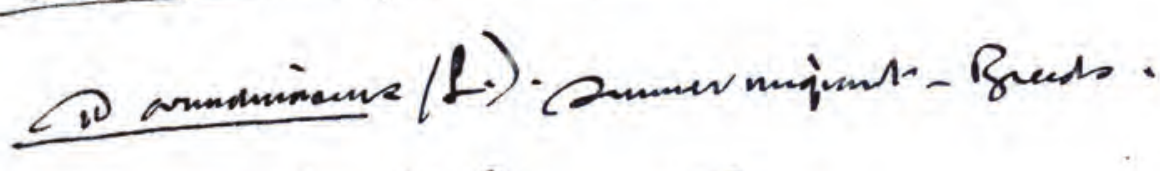

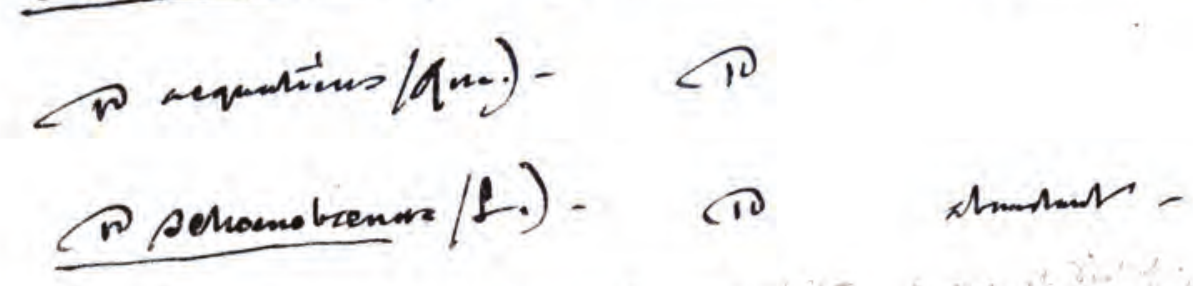

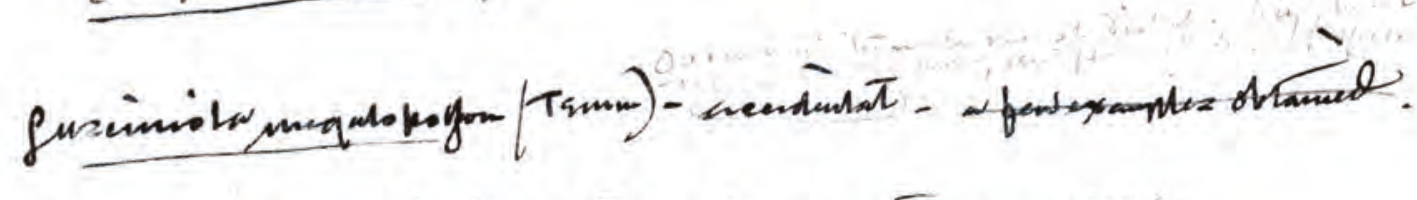

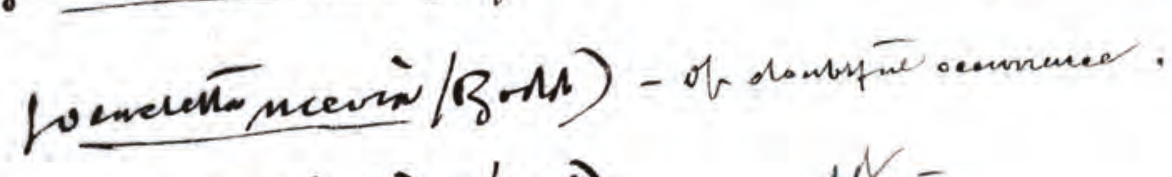

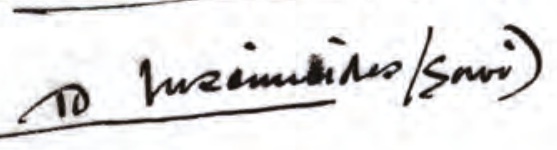

iN-

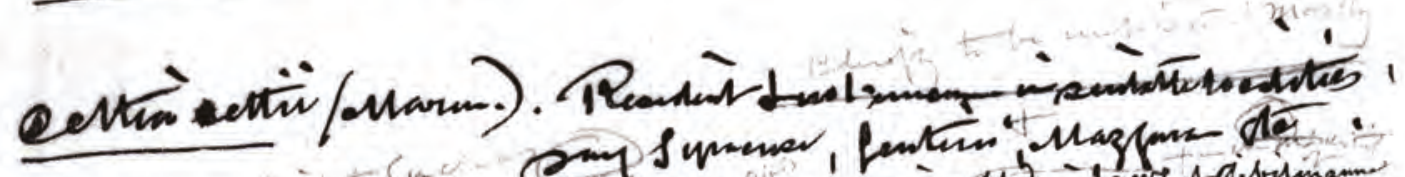

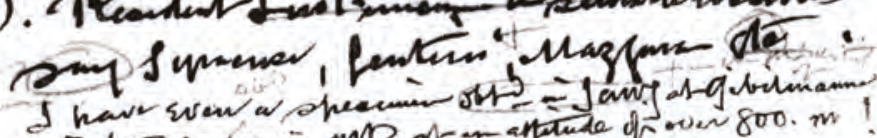

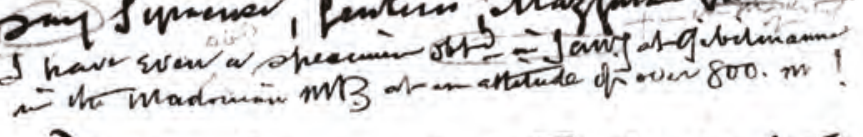

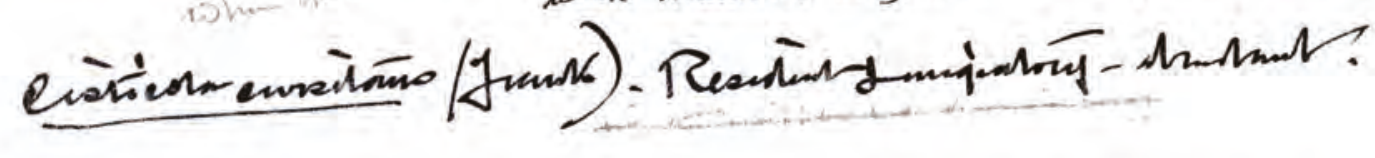

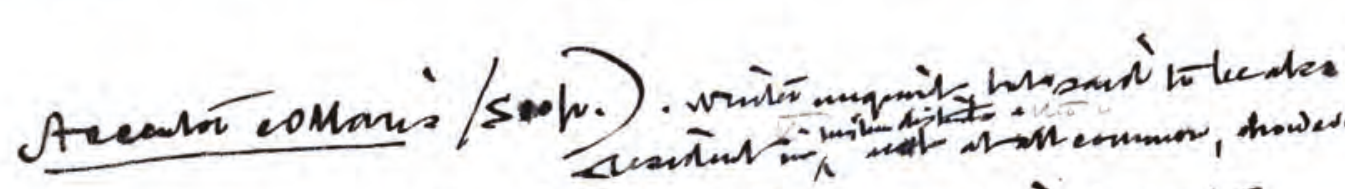

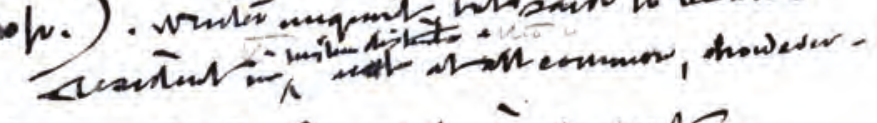

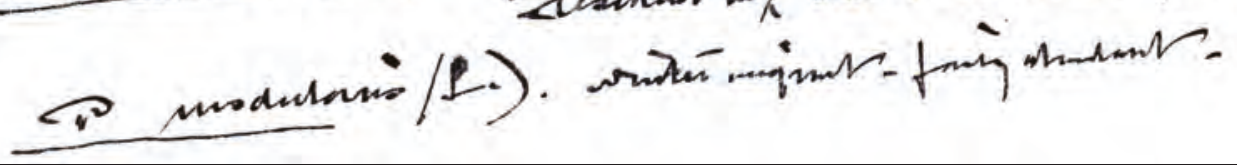

Figure 21. Page 4 of Birds of Sicily by Joseph Whitaker (ca. 1920). 


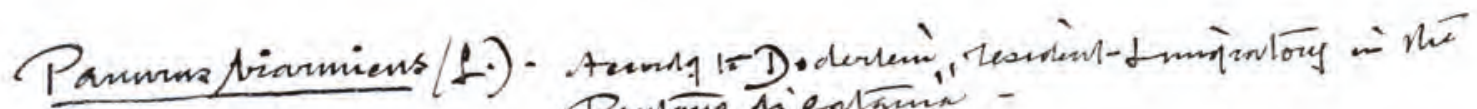
Pantano inentain -

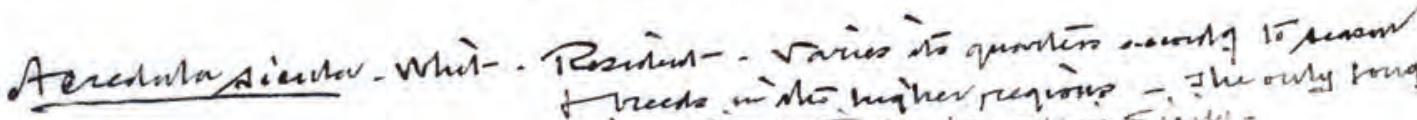

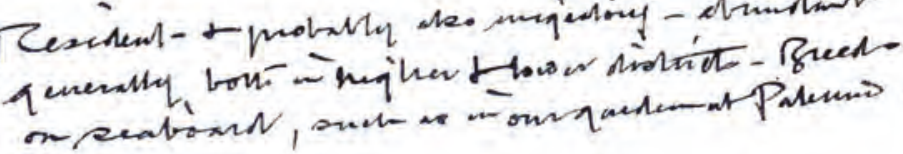

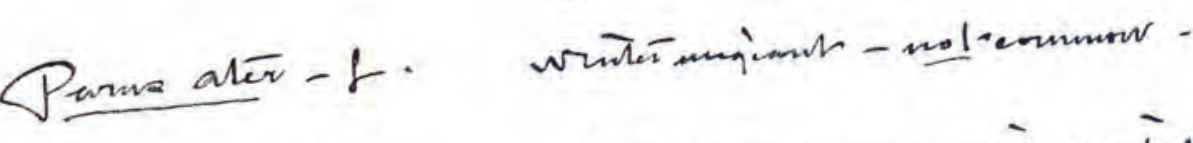

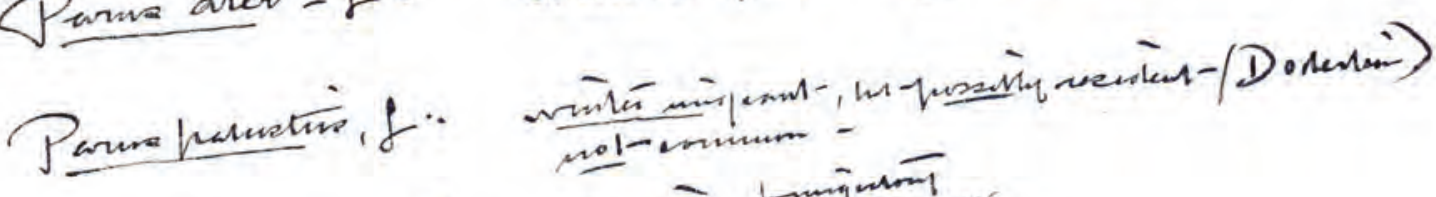

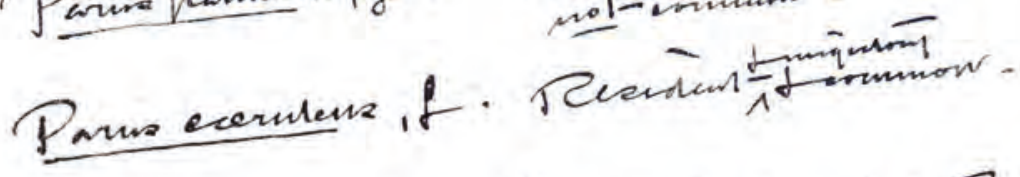

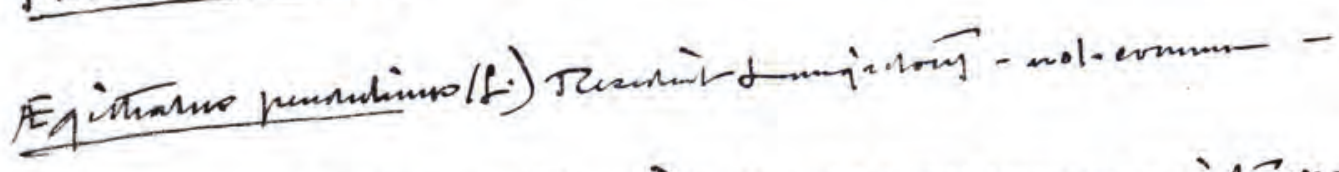

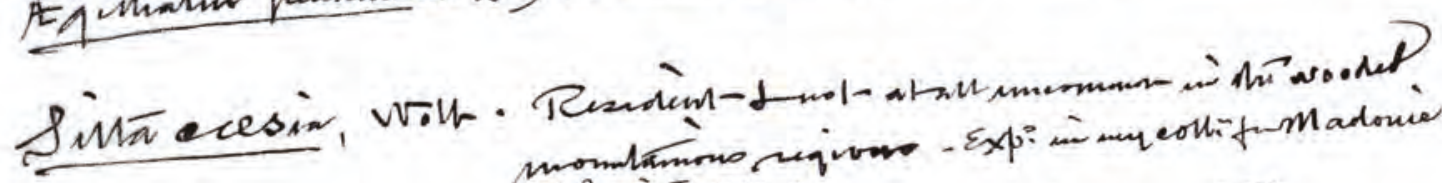

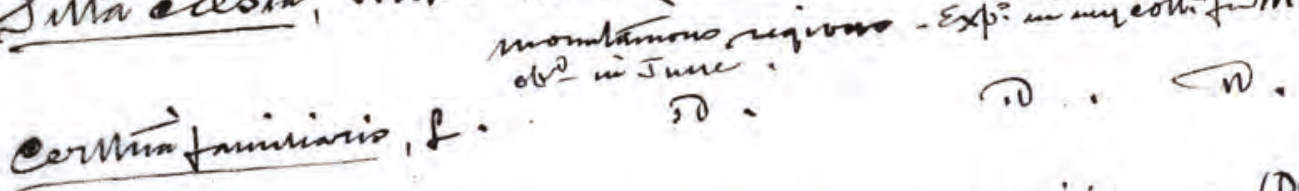

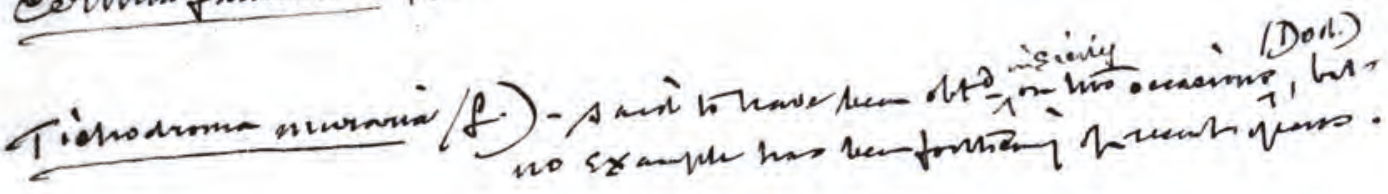

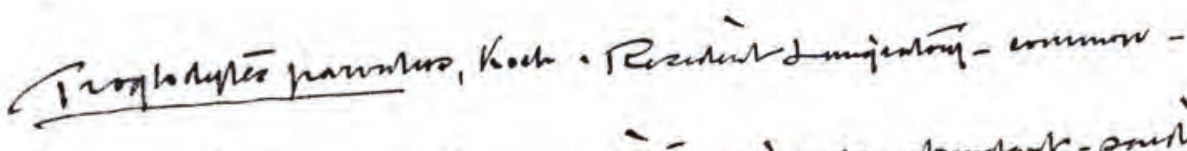

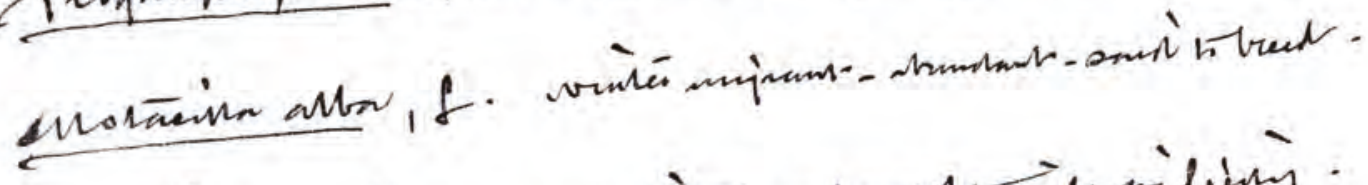

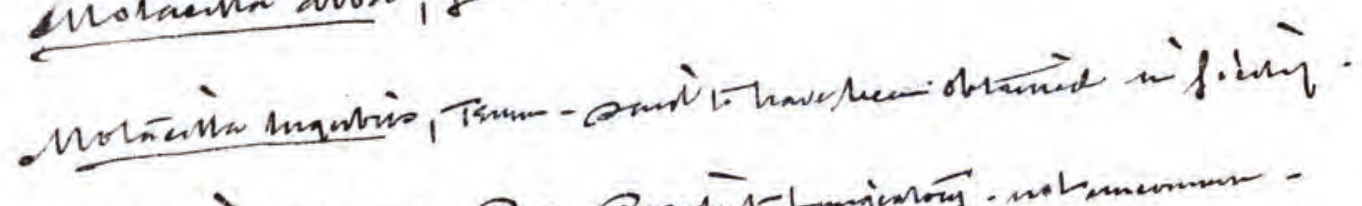

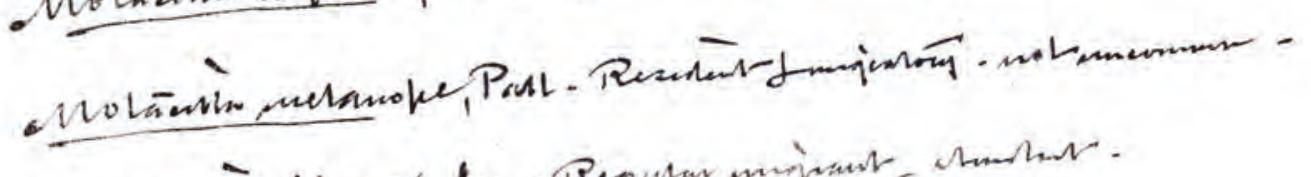

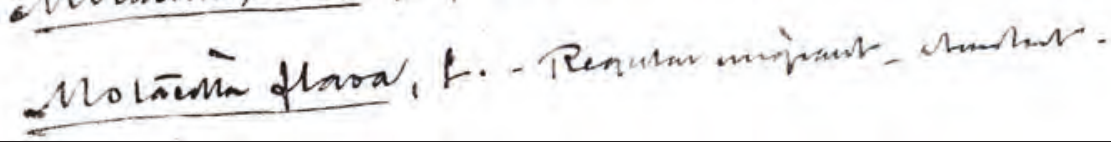

Figure 22. Page 5 of Birds of Sicily by Joseph Whitaker (ca. 1920). 


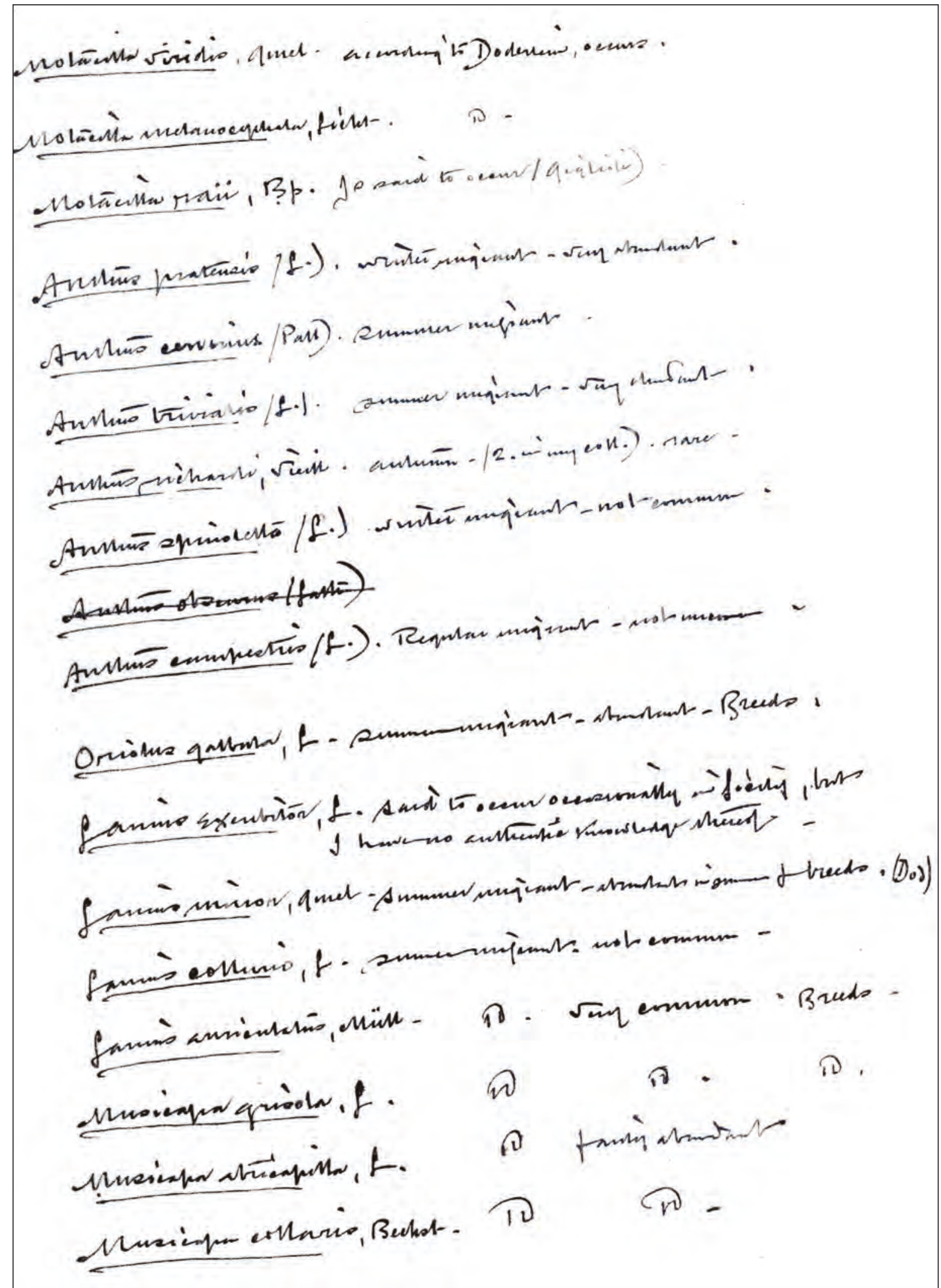

Figure 23. Page 6 of Birds of Sicily by Joseph Whitaker (ca. 1920). 


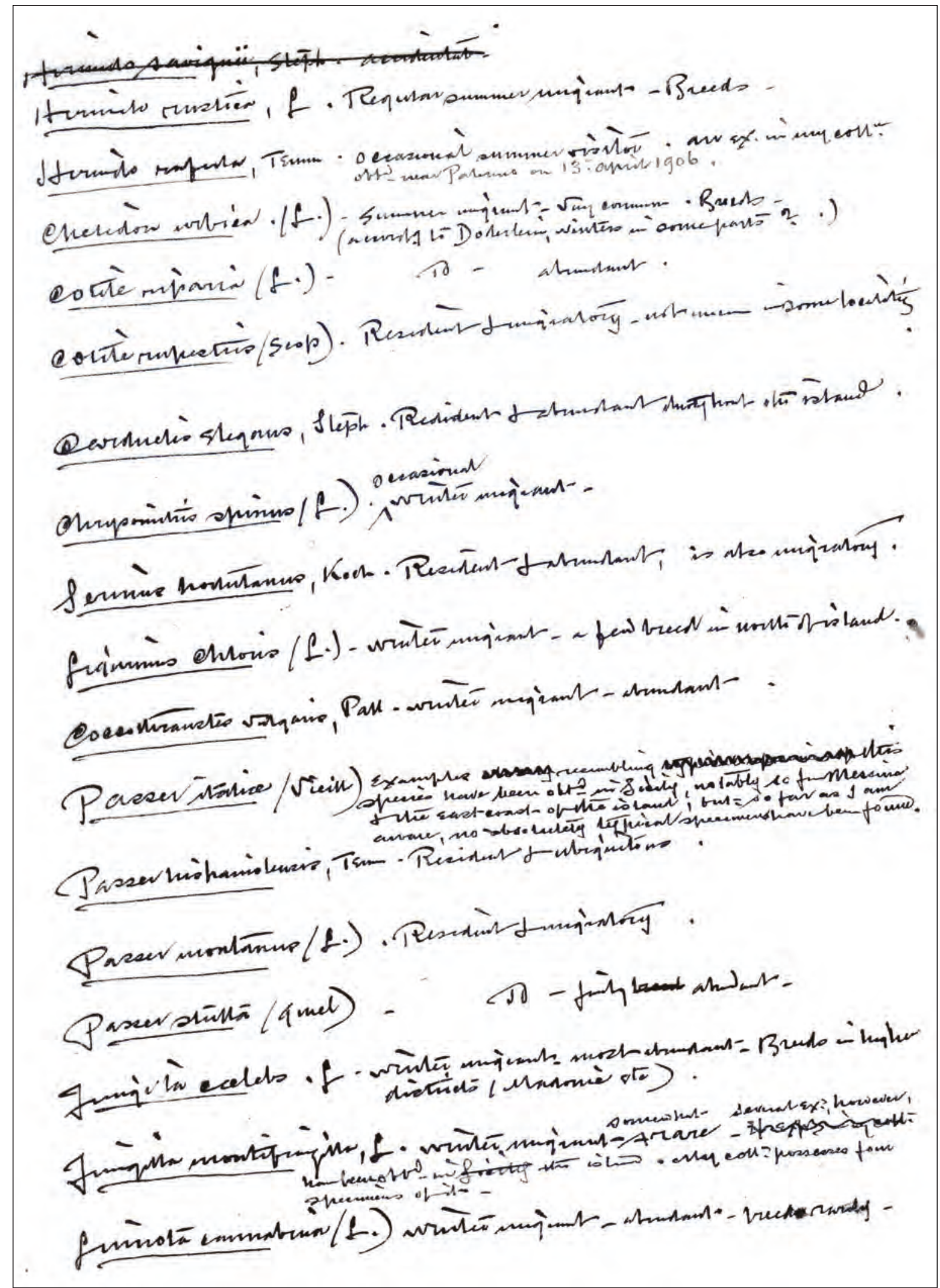

Figure 24. Page 7 of Birds of Sicily by Joseph Whitaker (ca. 1920). 


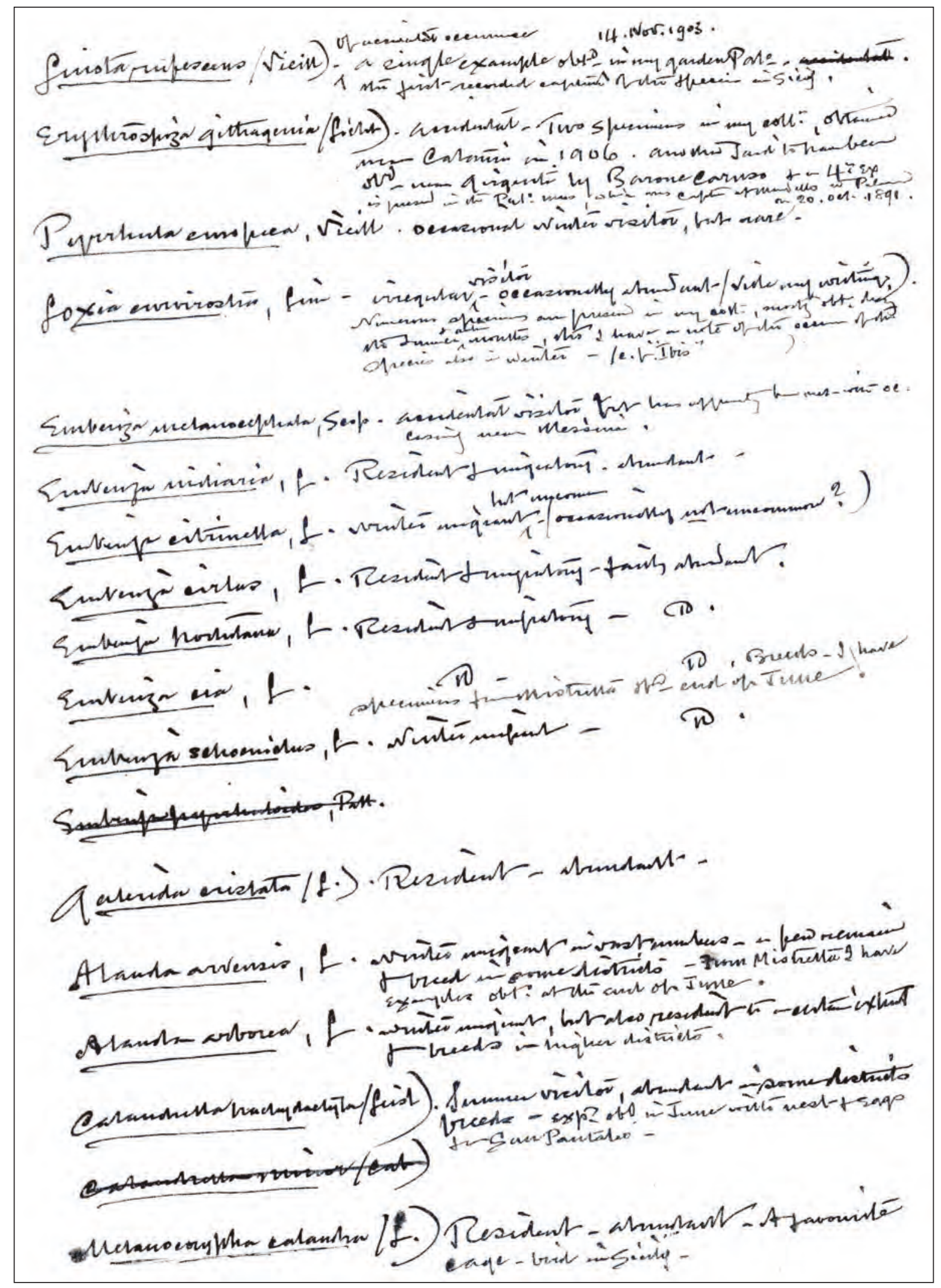

Figure 25. Page 8 of Birds of Sicily by Joseph Whitaker (ca. 1920). 


\section{(3) -}

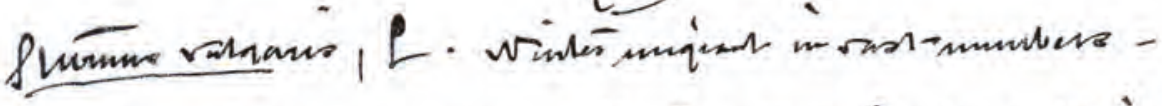

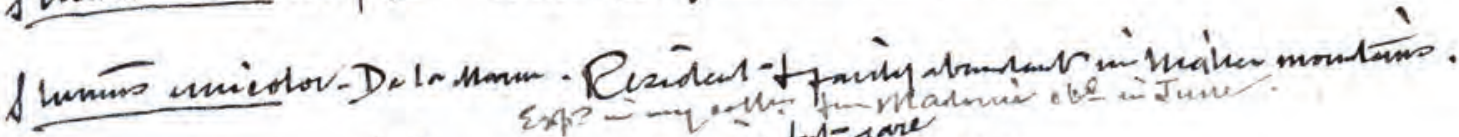

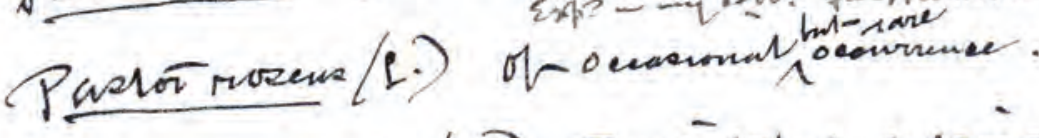

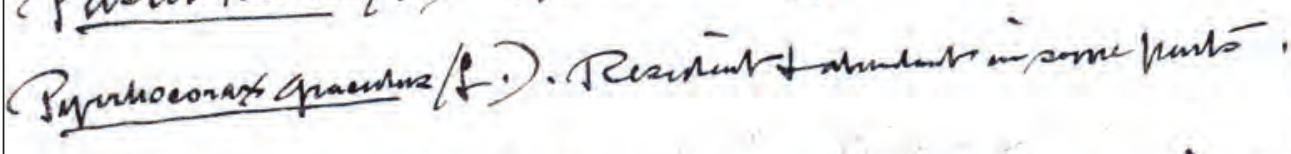

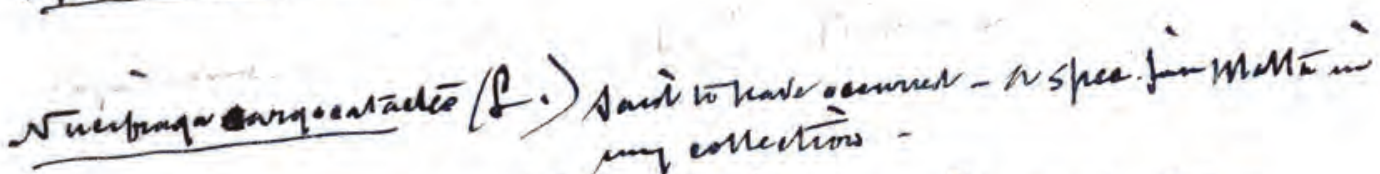

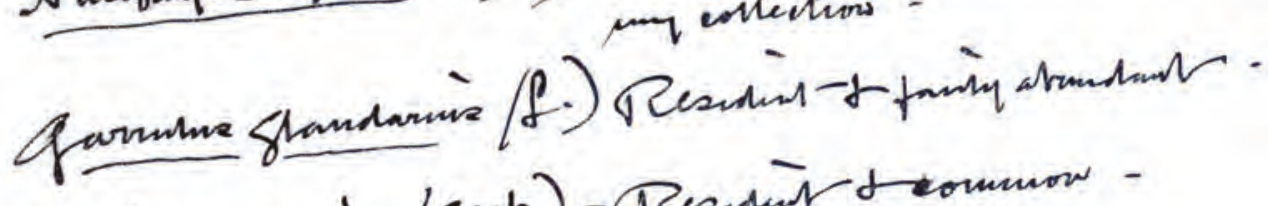
Pien rustien (sepp) - TCesdut d common -

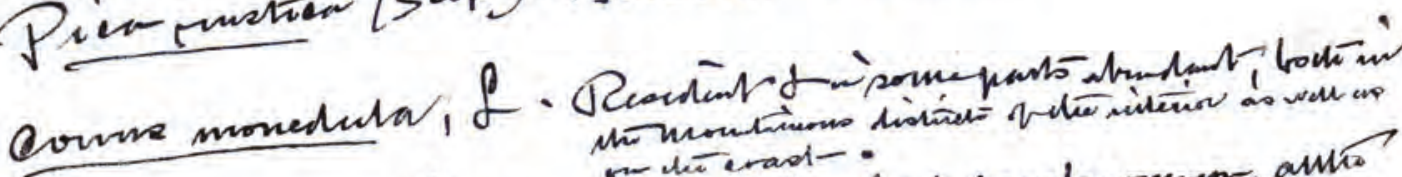

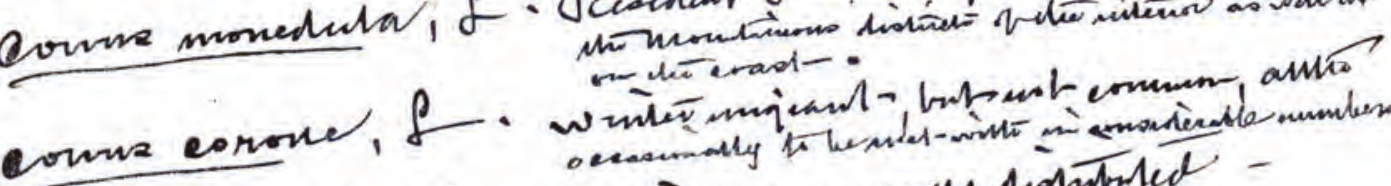

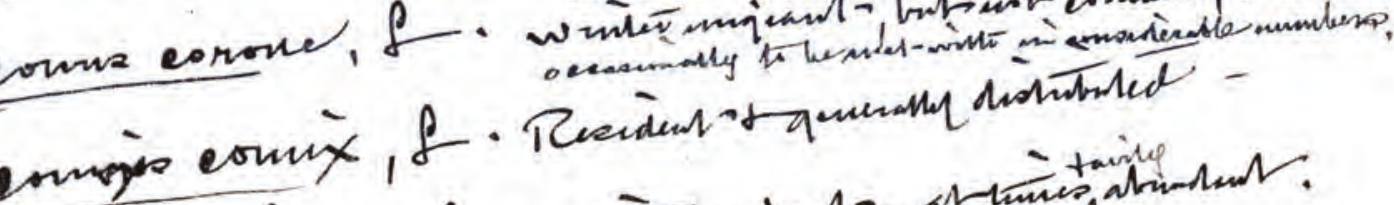

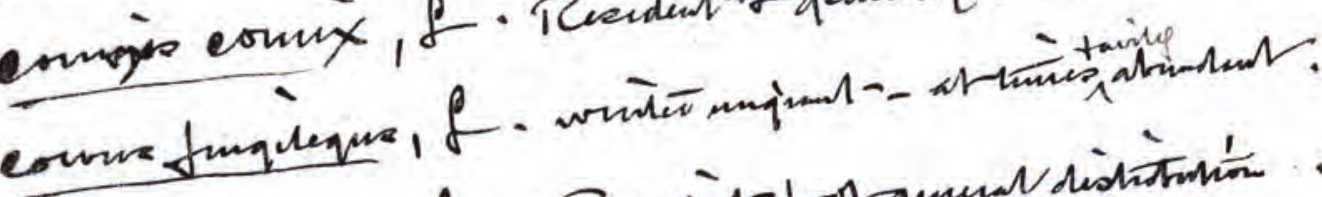

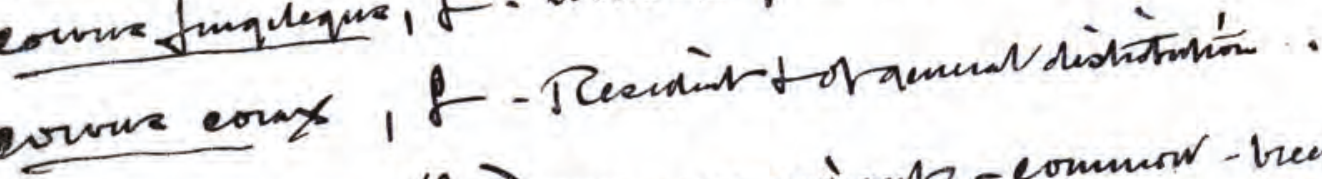

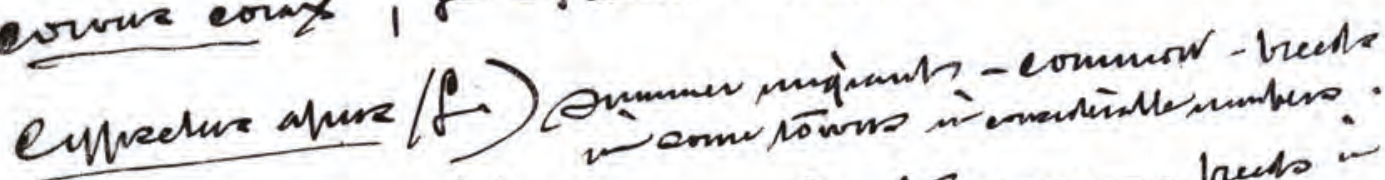

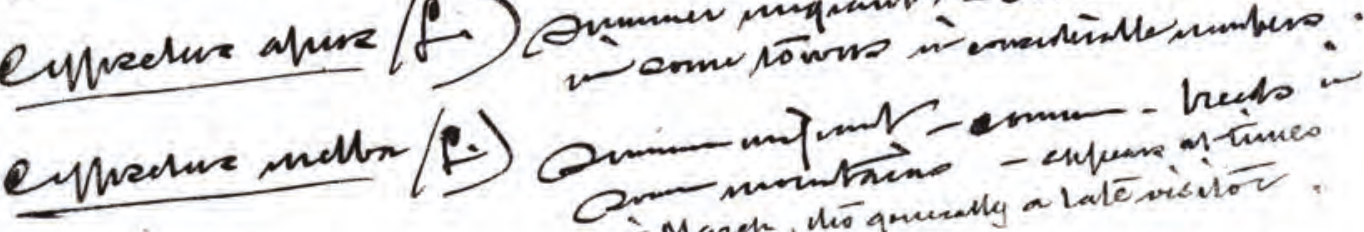

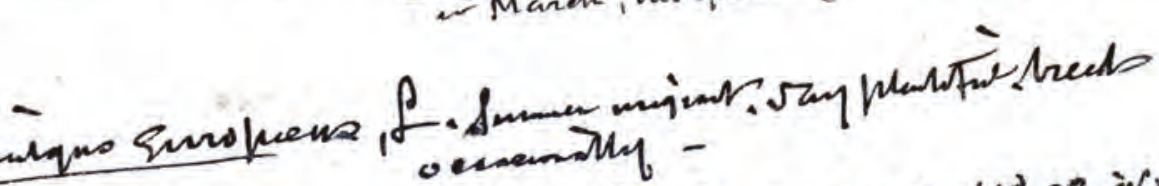
cecsemally -

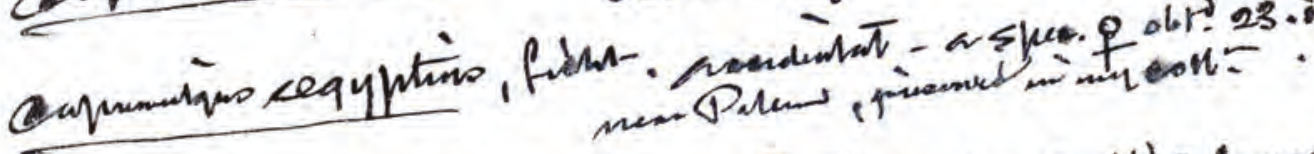

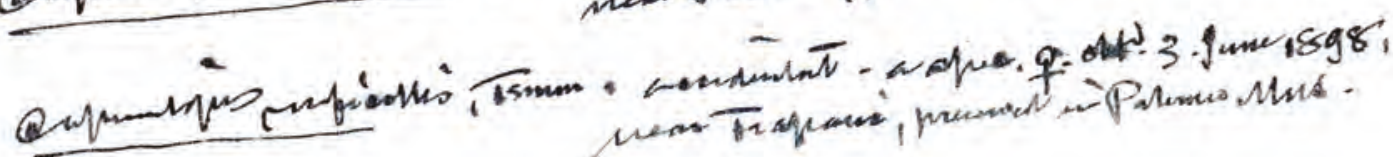




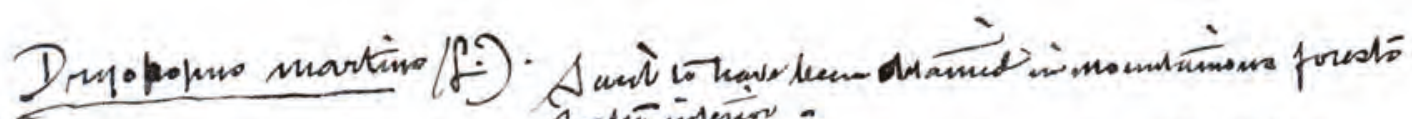

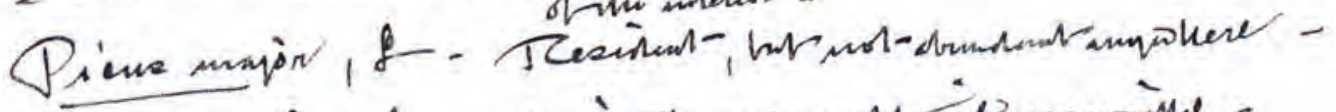

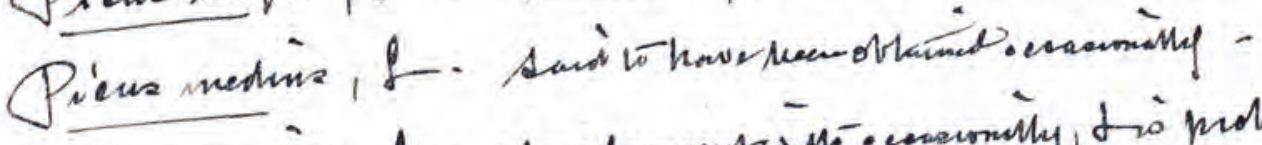

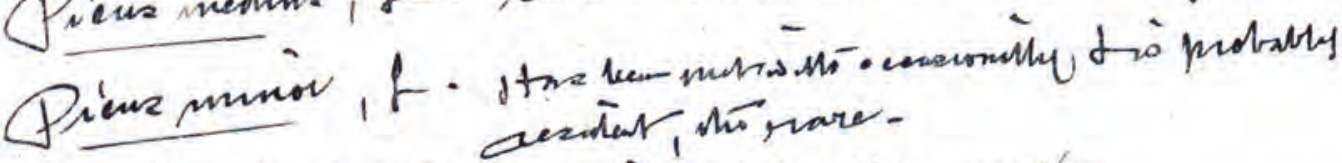

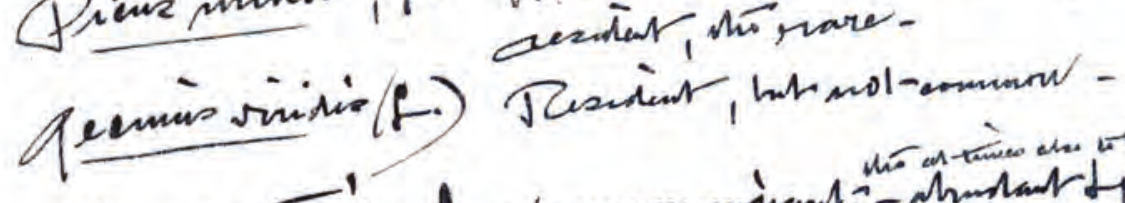

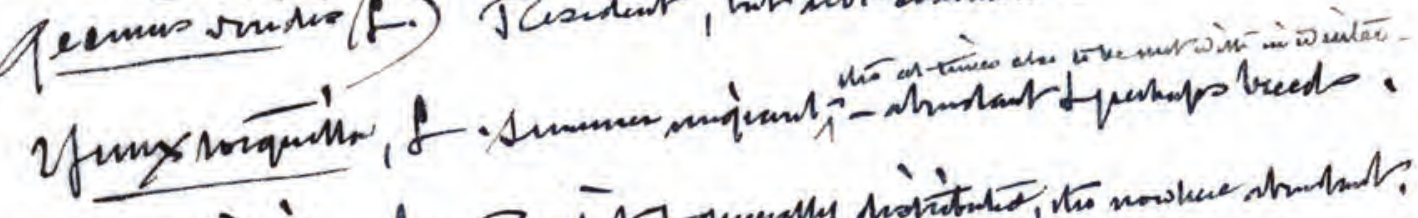

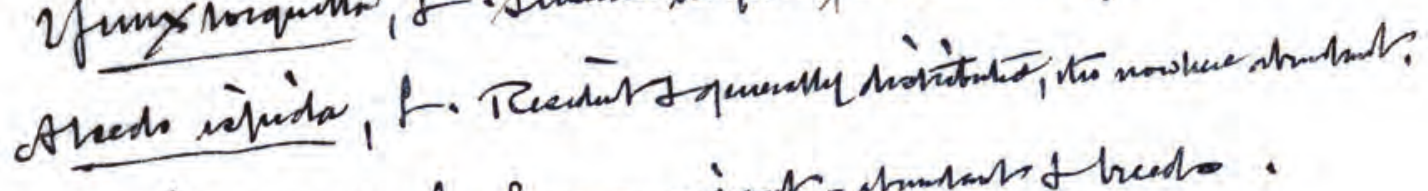

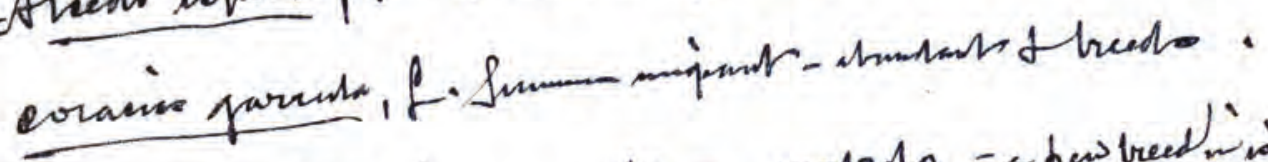

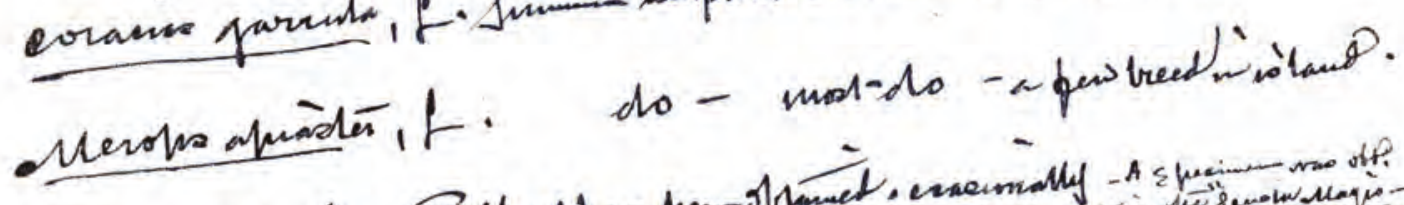

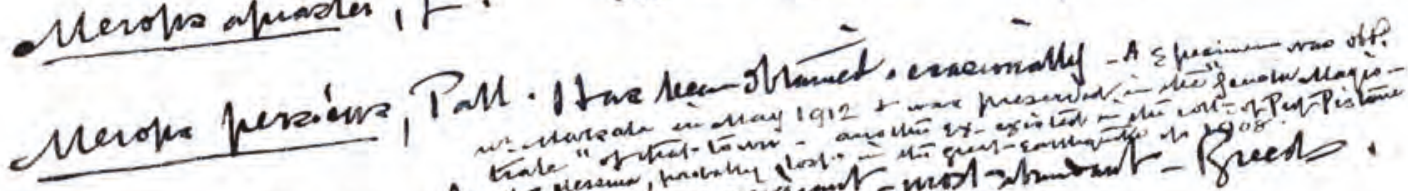

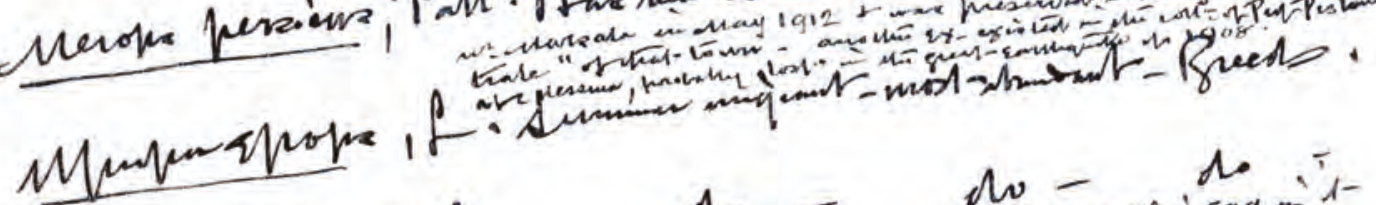

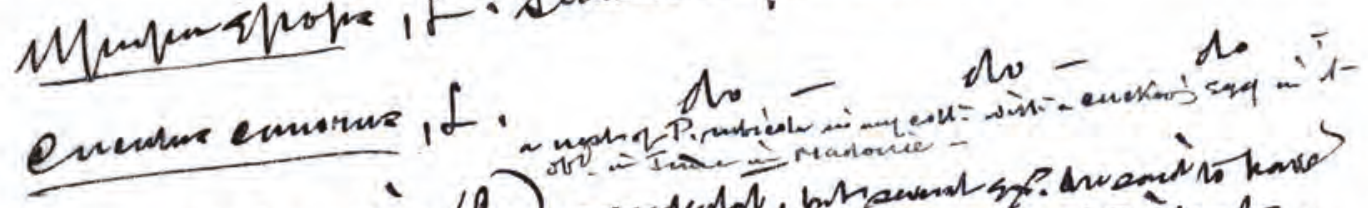

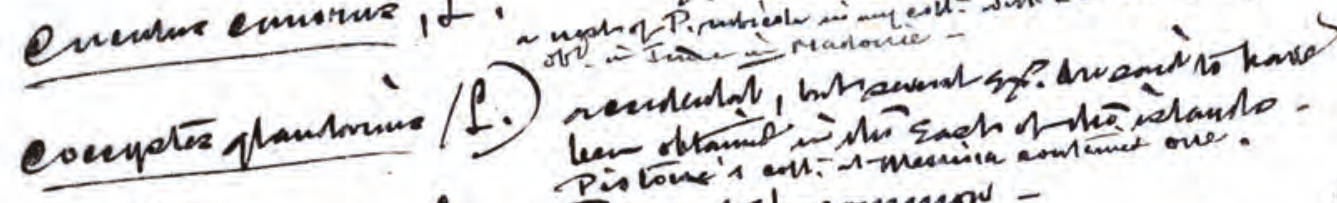

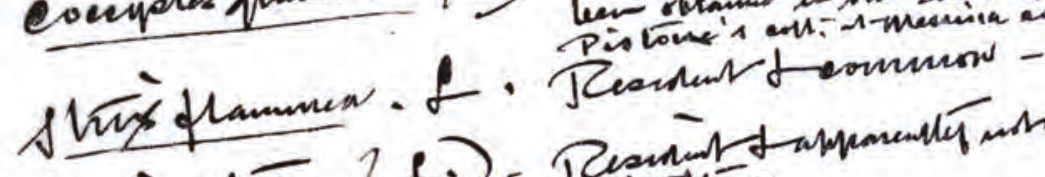

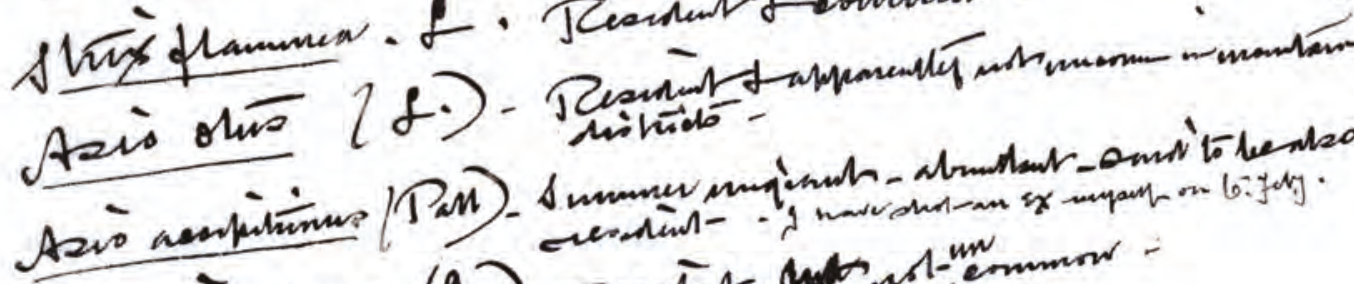

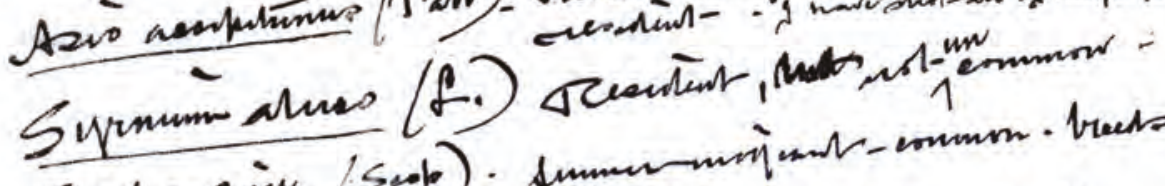

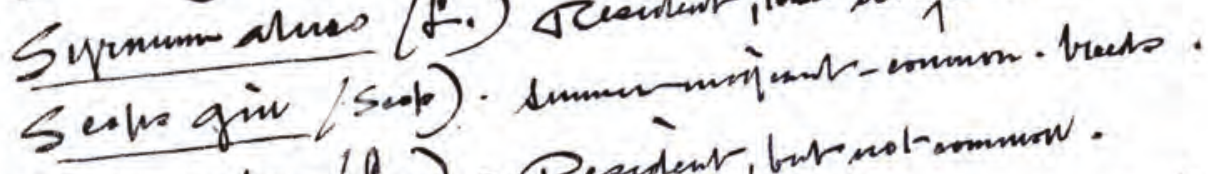

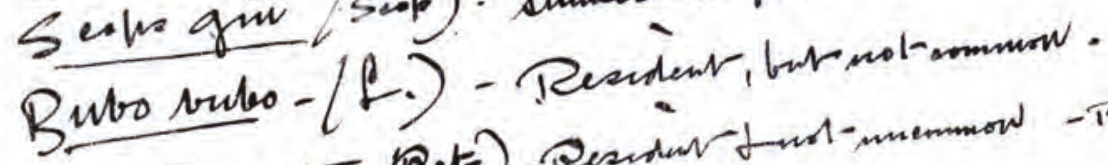

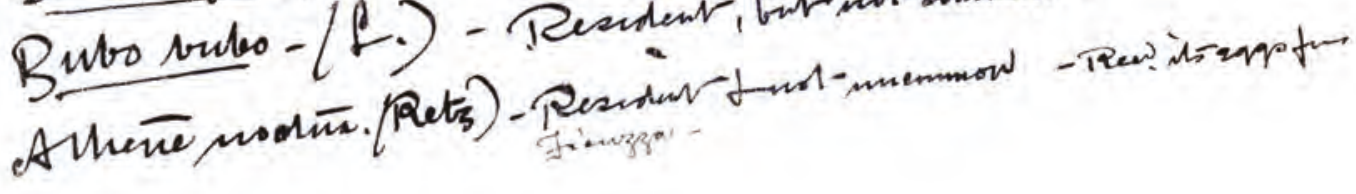




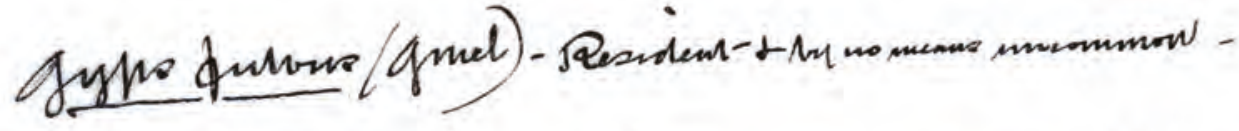

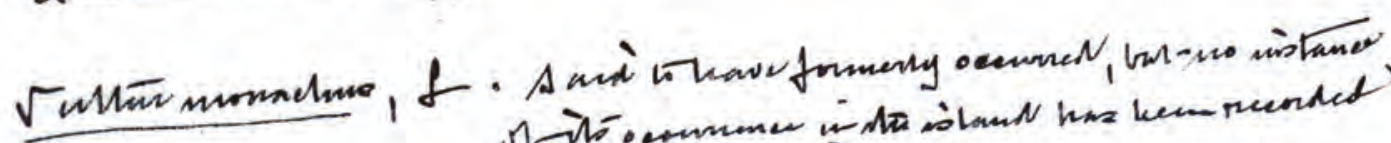

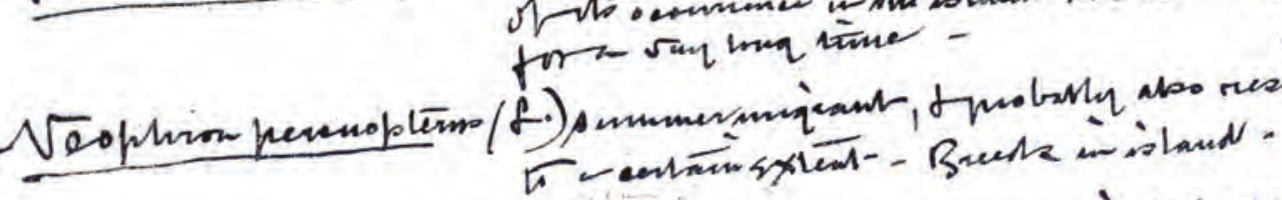

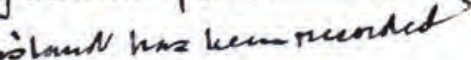

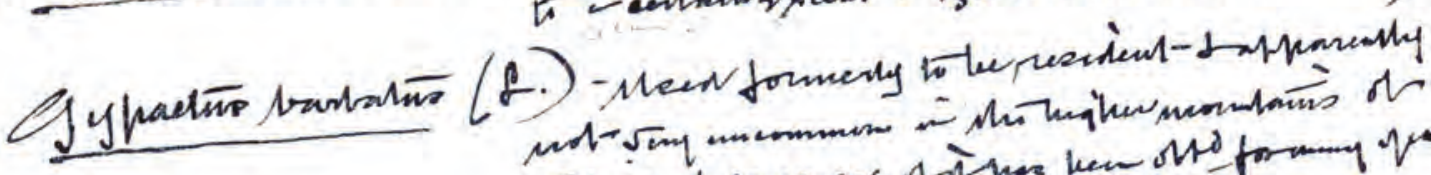

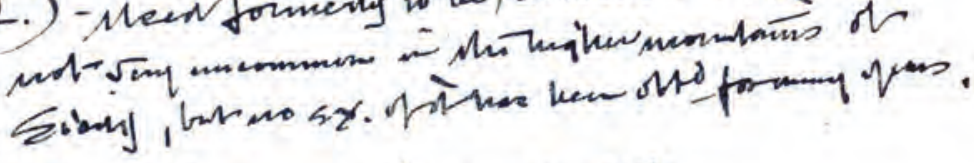

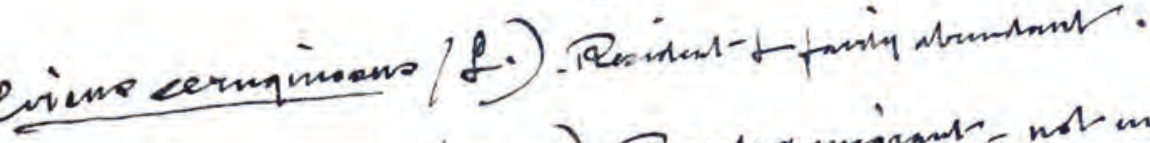

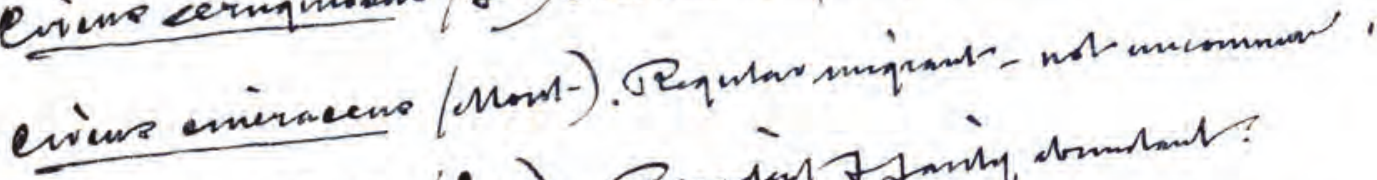

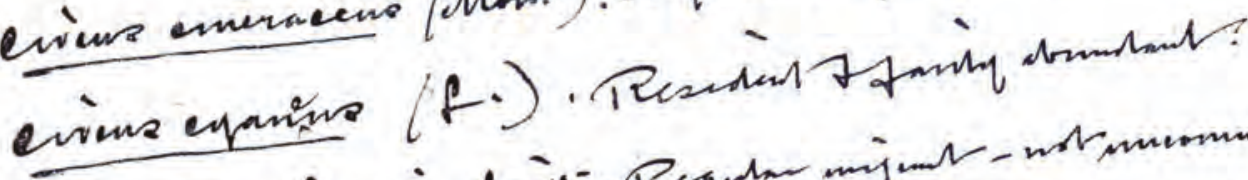

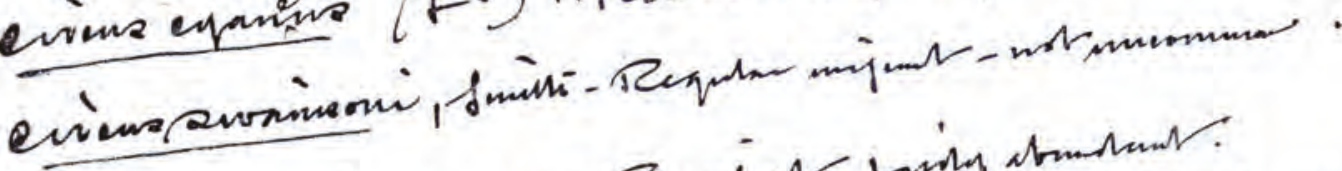

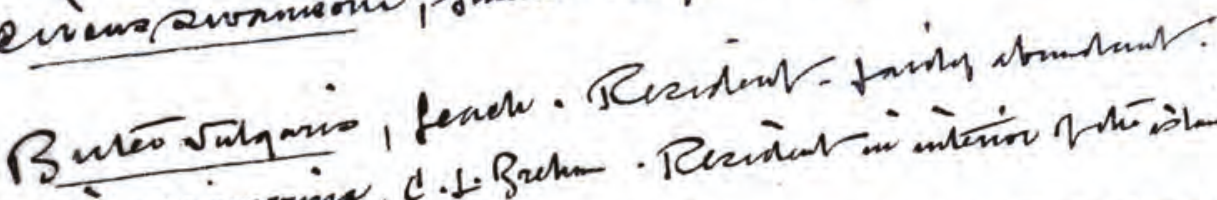

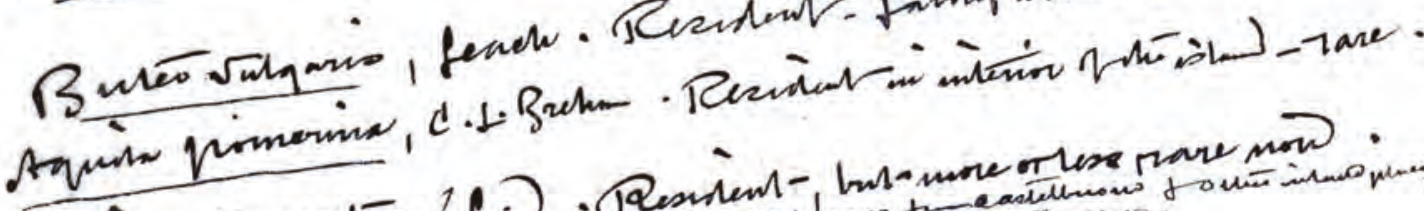

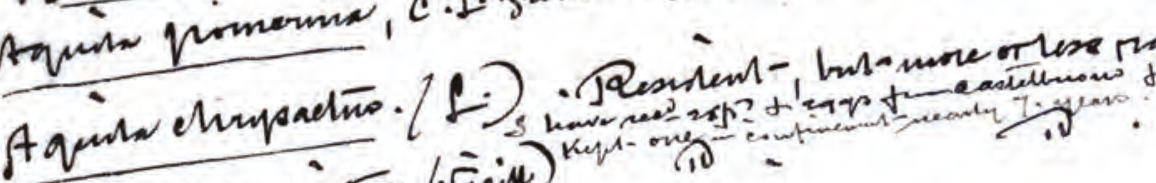

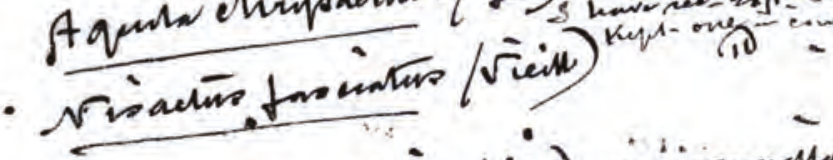

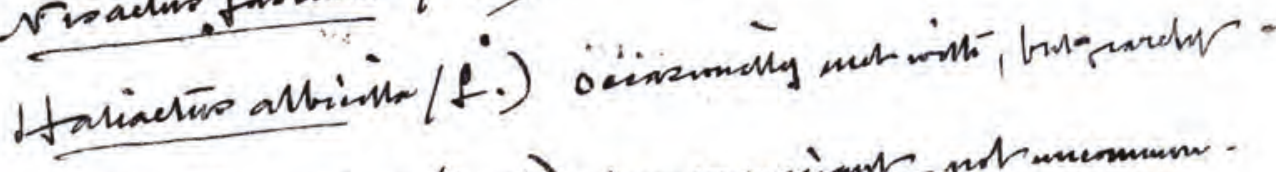

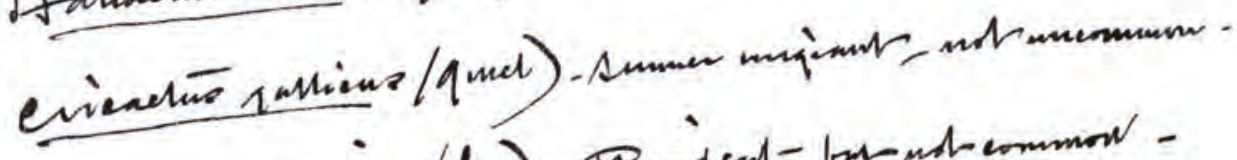

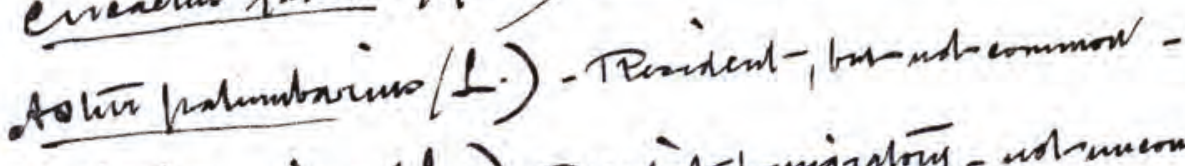

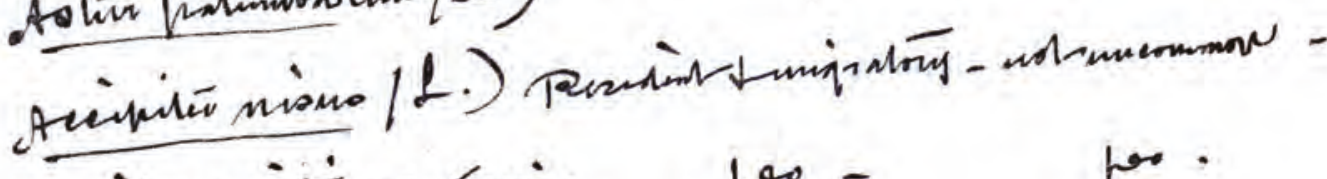
Mitore sitimur, savig. bo - her.

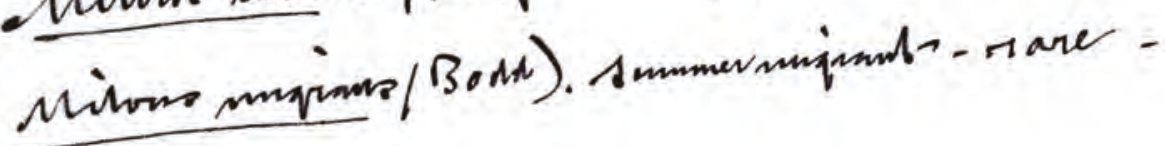




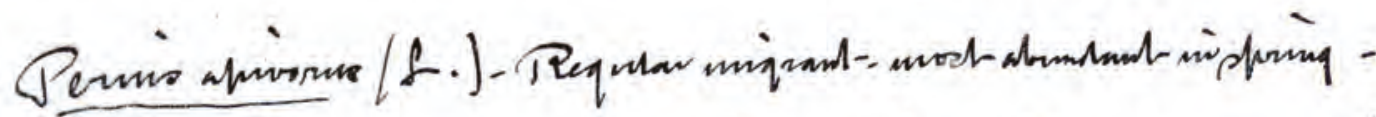

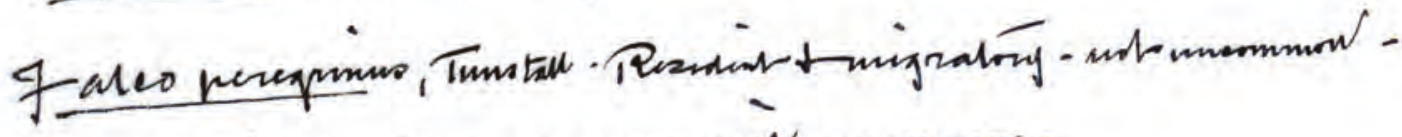

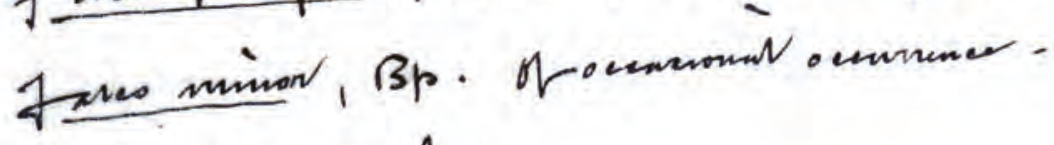

Janeo barbans, $f$.

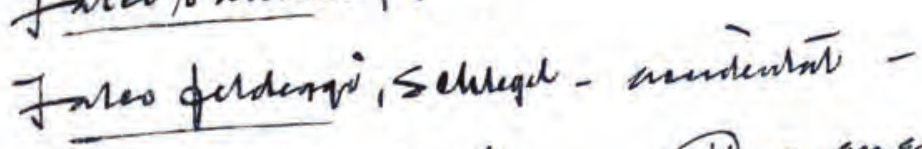

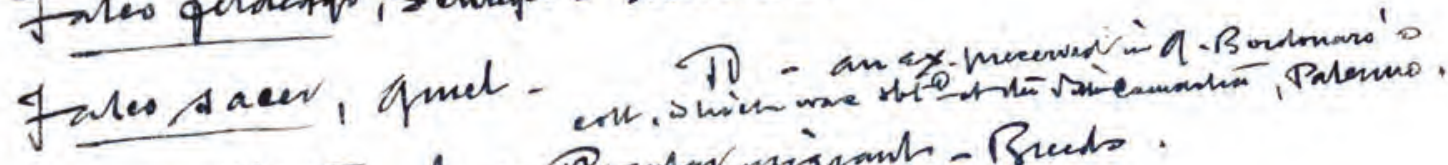
7 ares subbutés, f. Sequatar minant-Breets.

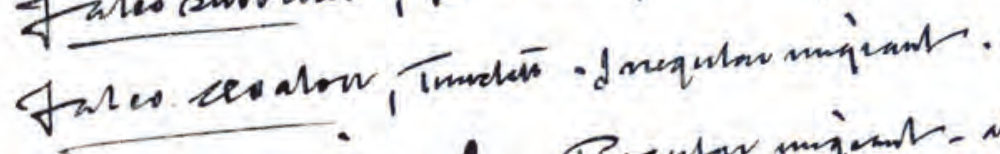

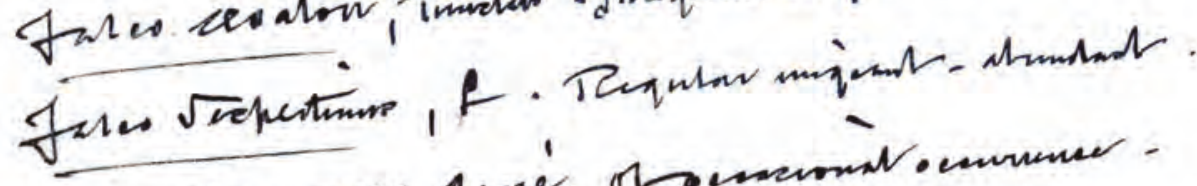

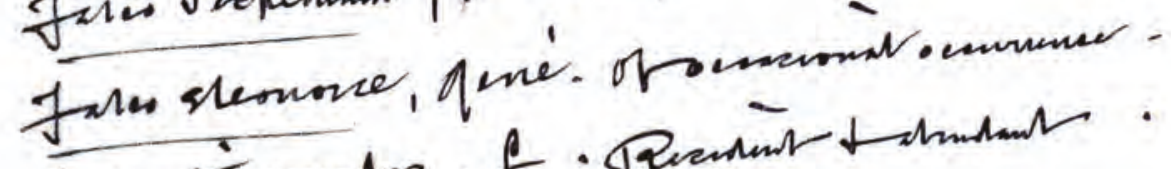

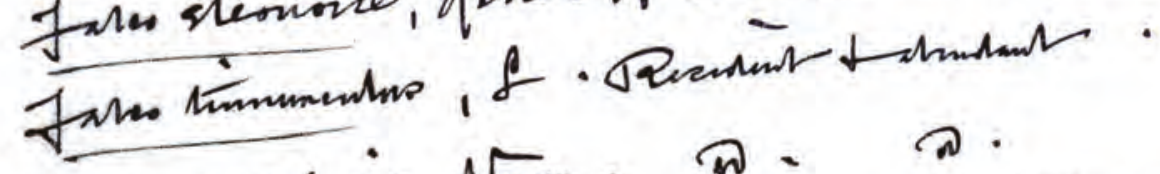

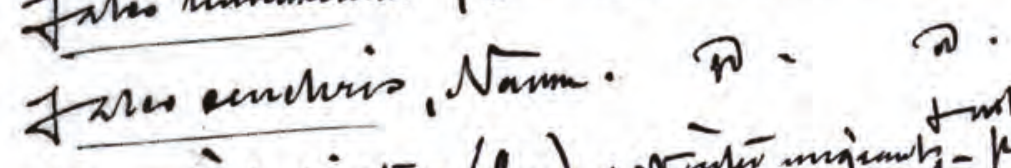

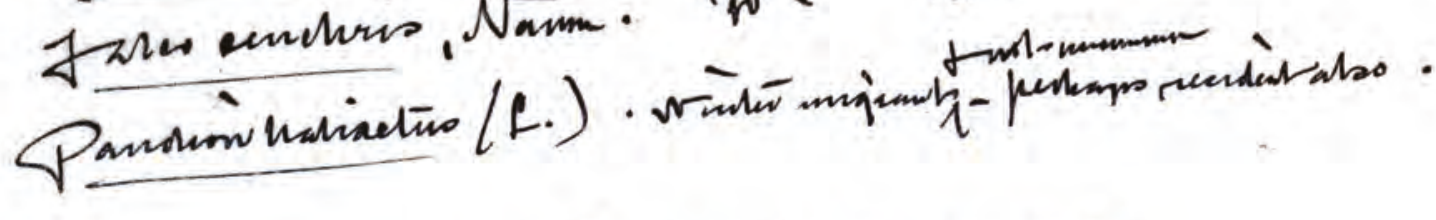

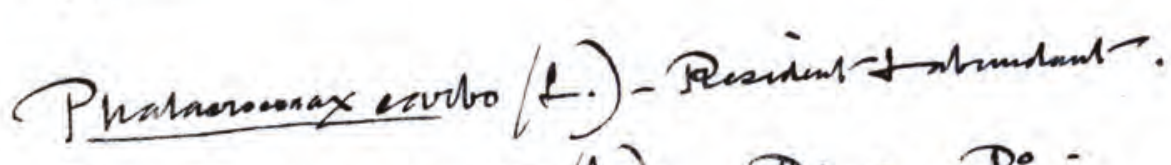

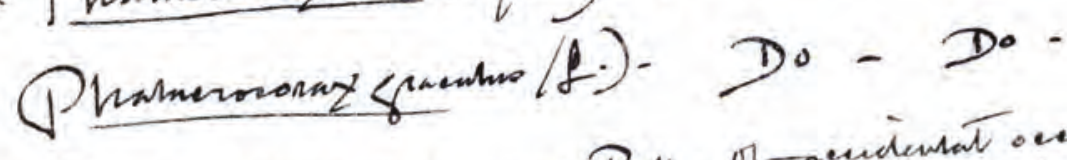

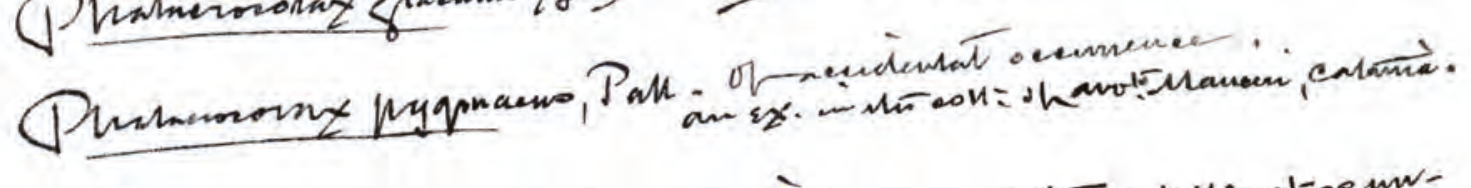

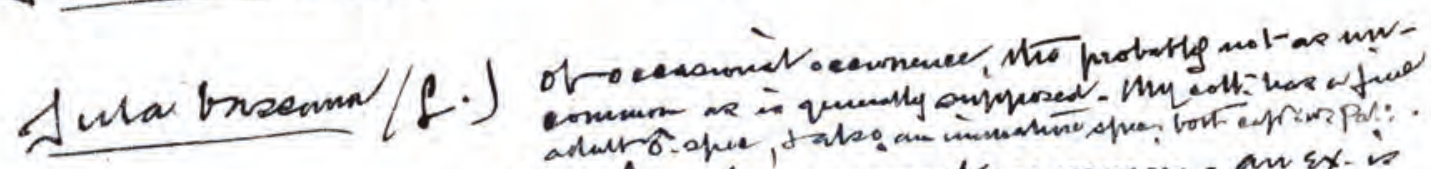

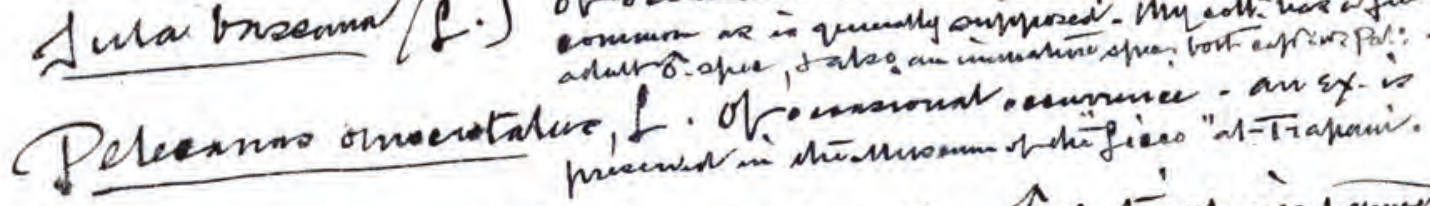

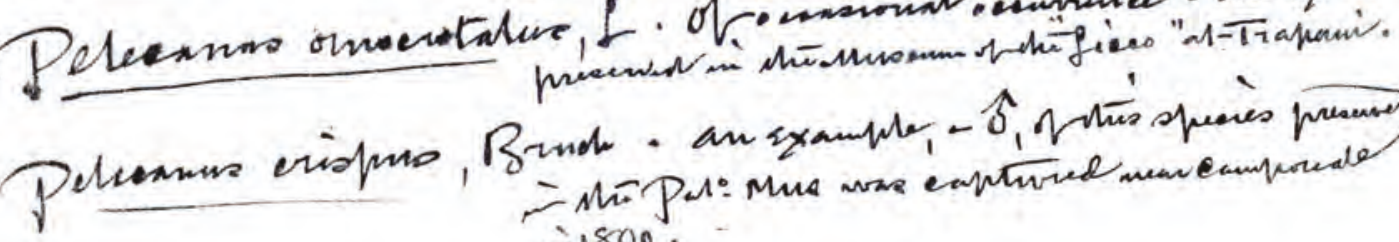




\section{(4)}

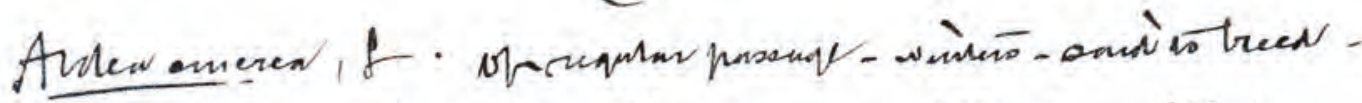

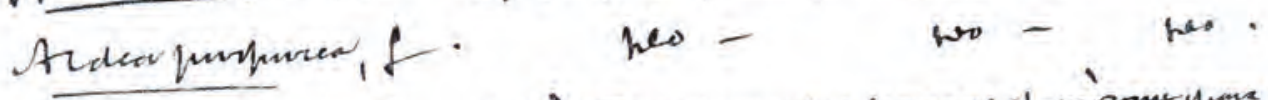

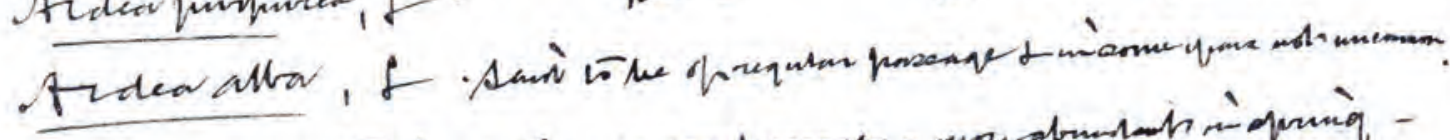

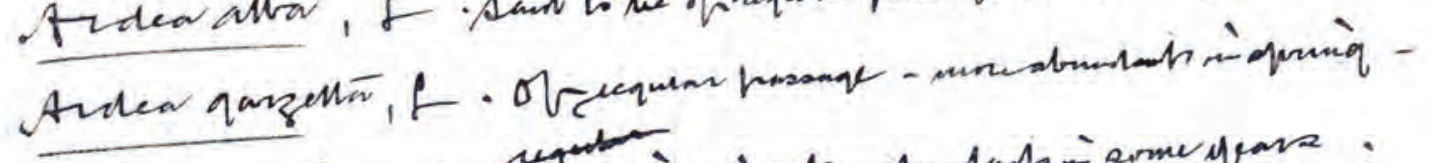

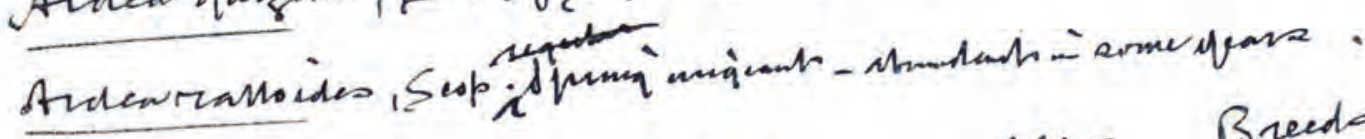

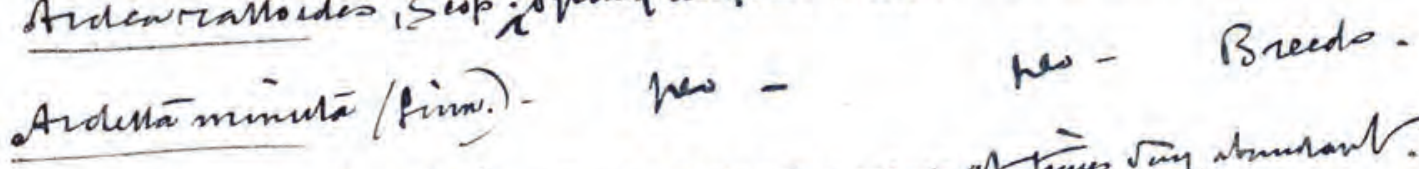

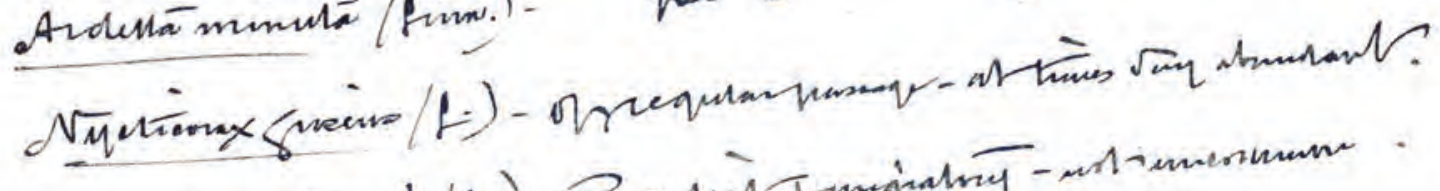

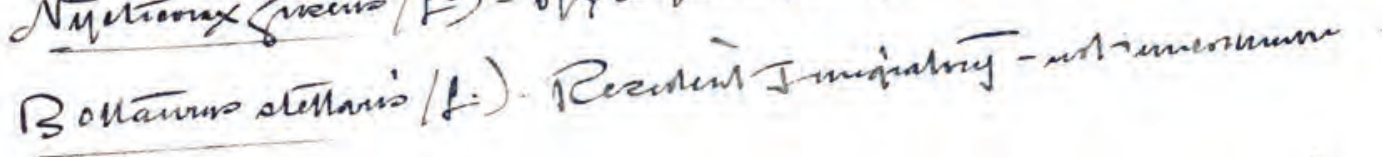

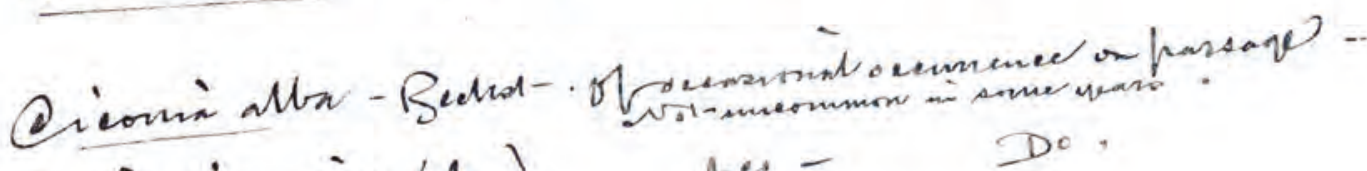
encominavia lf.) De.

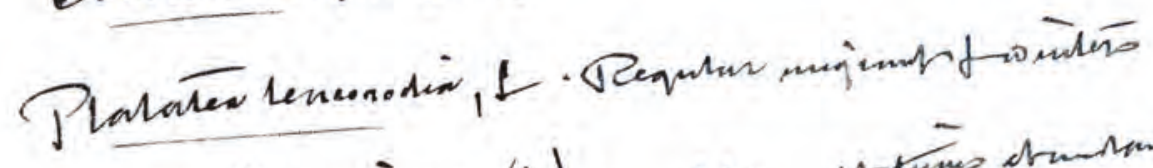

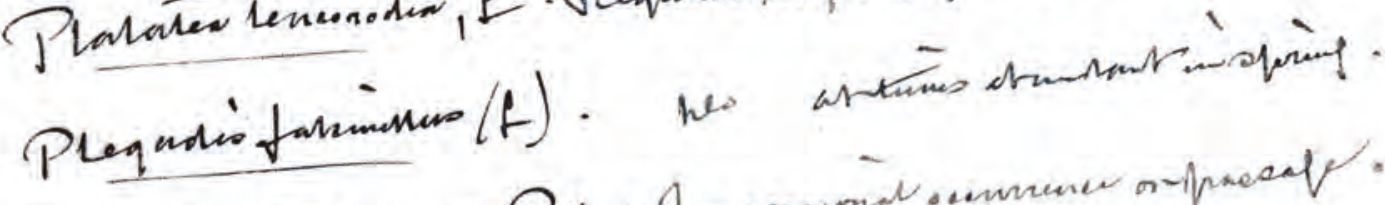

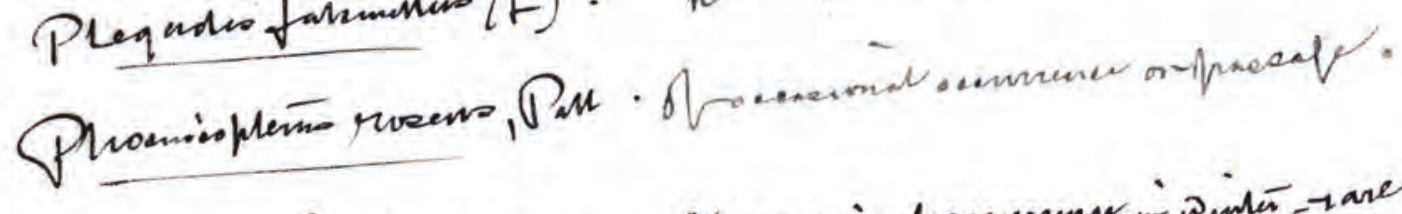

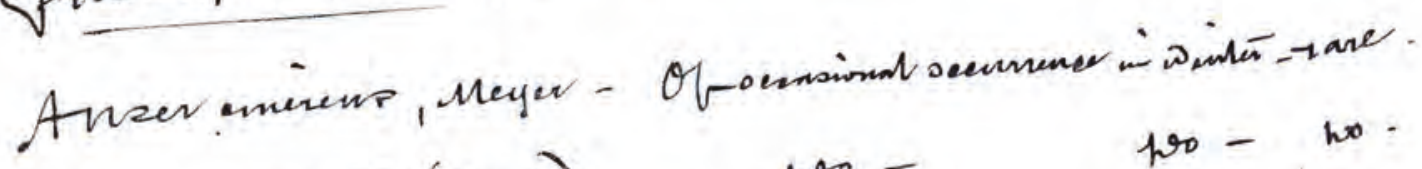

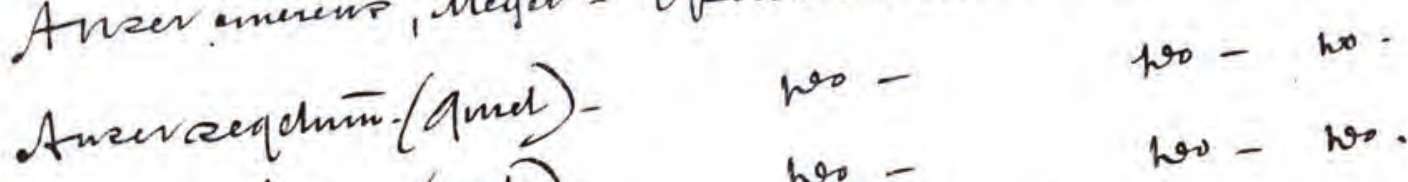
Aneer abifiome - scop. - wo bo - wo.

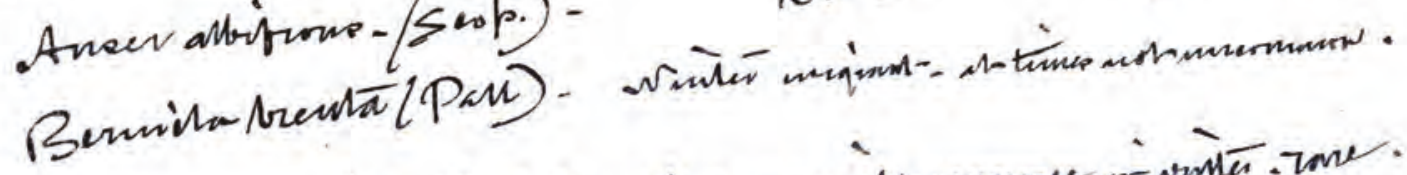

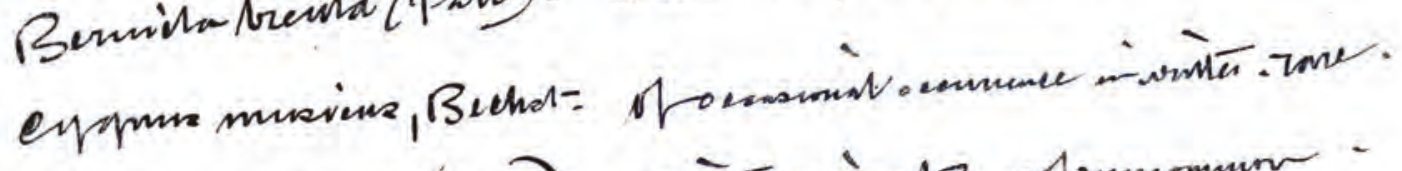

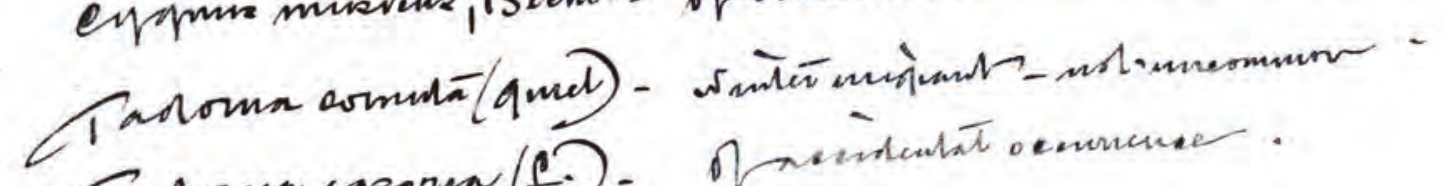

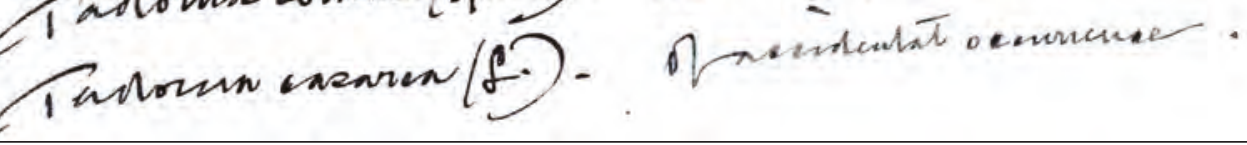




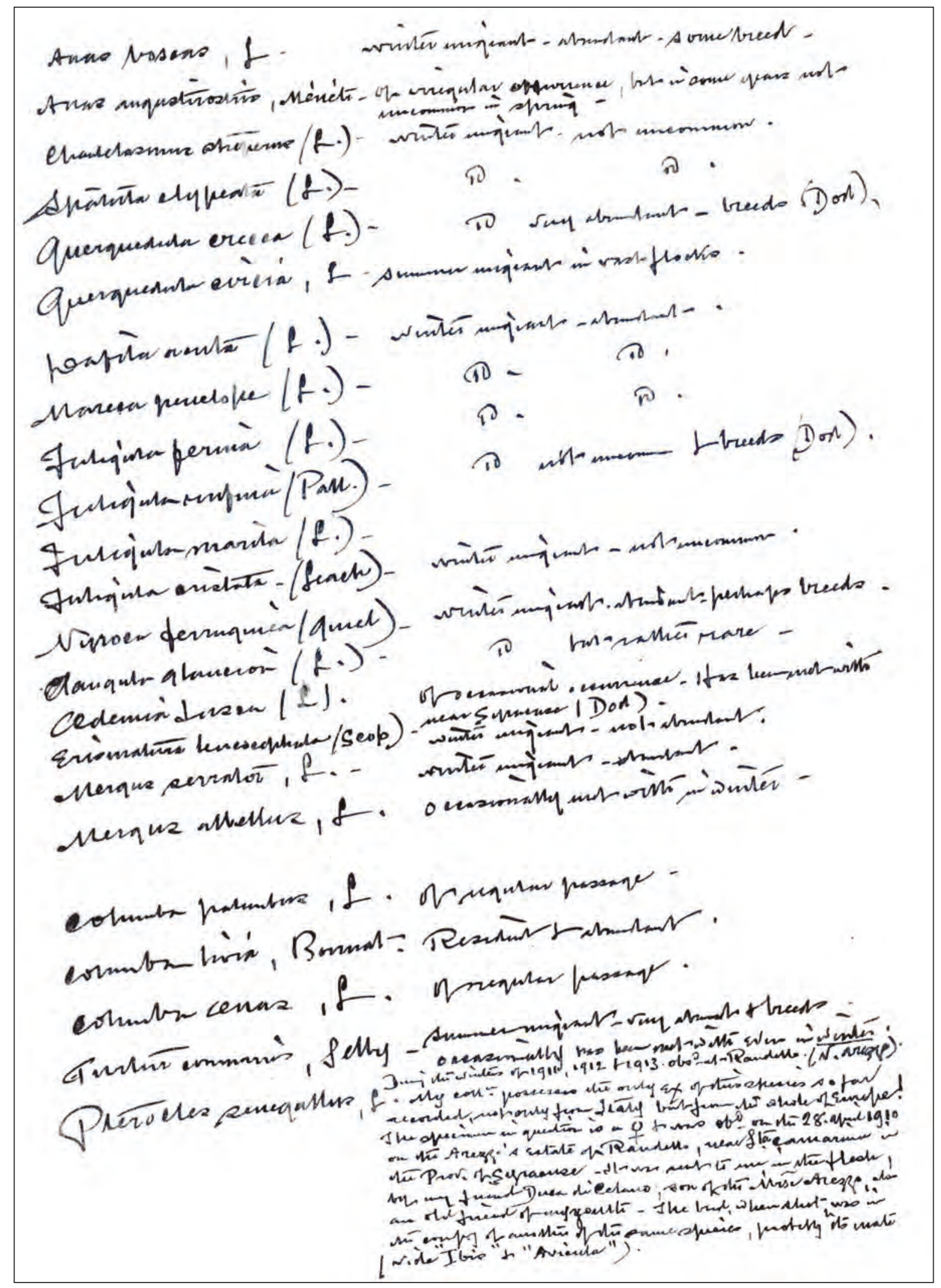




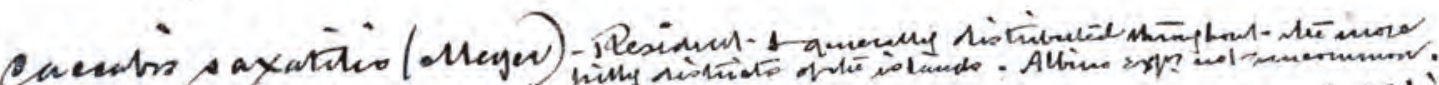

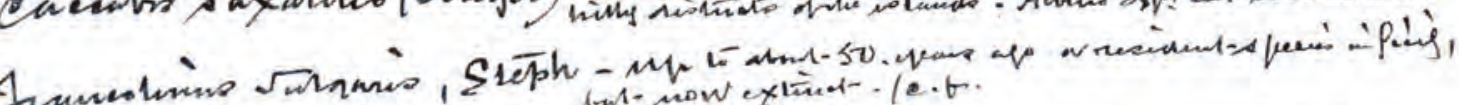

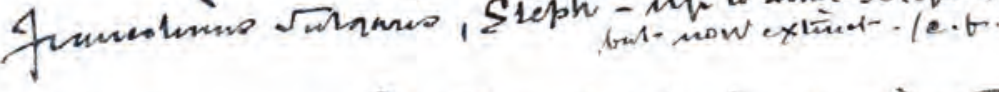

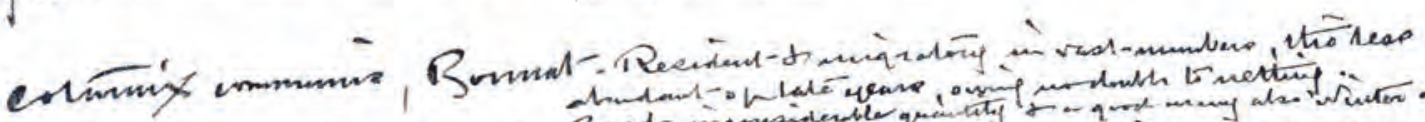

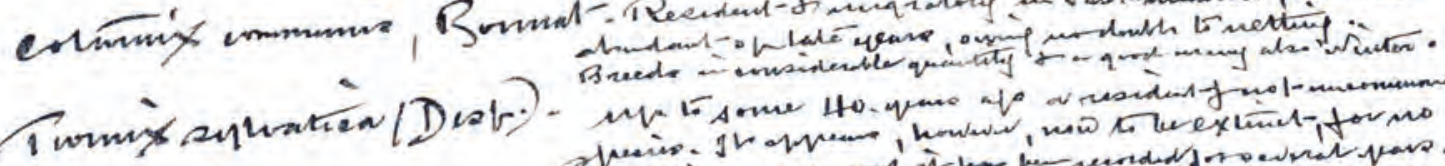

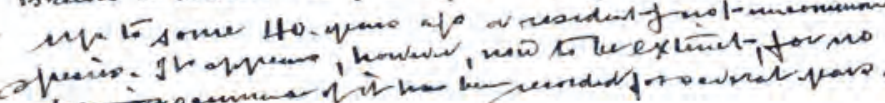

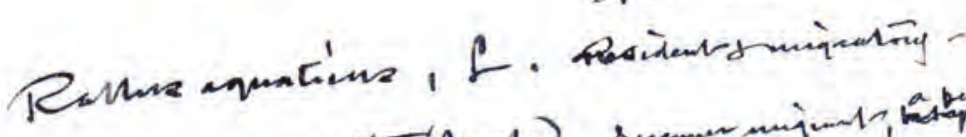

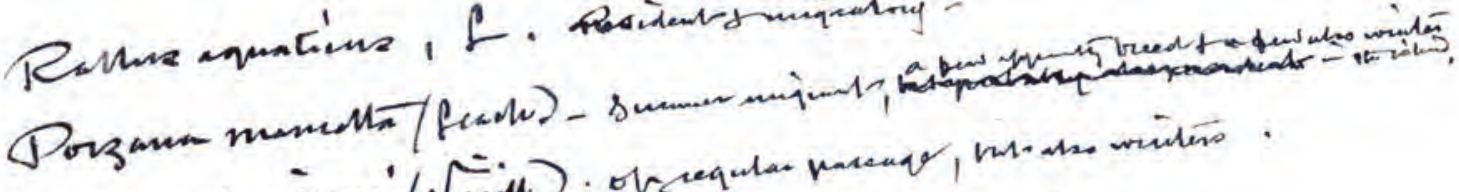

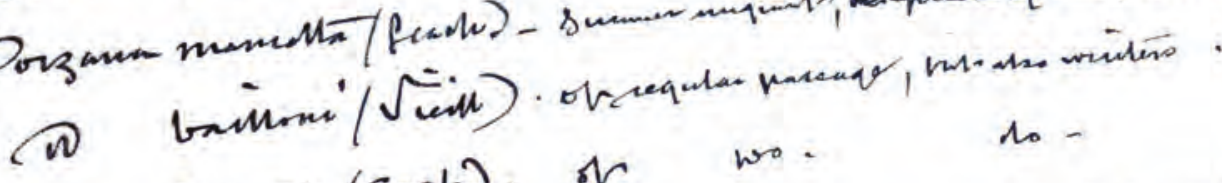

(10 hanor (Se0p). of wo. to

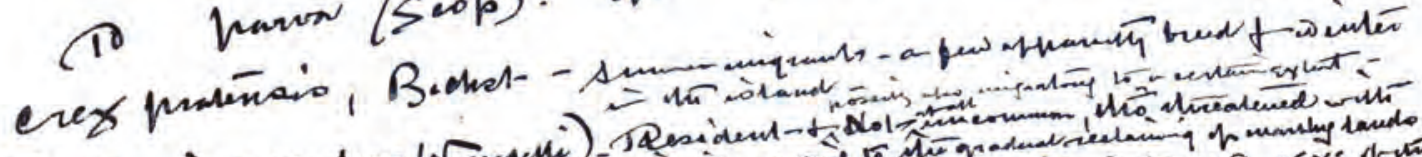

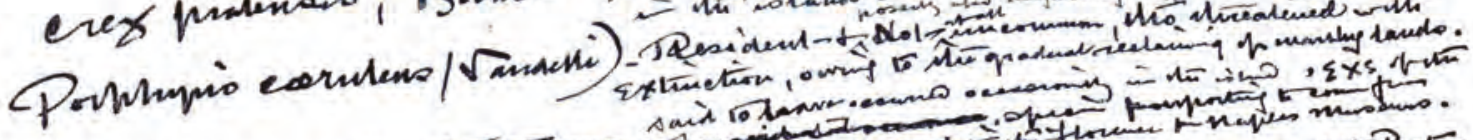

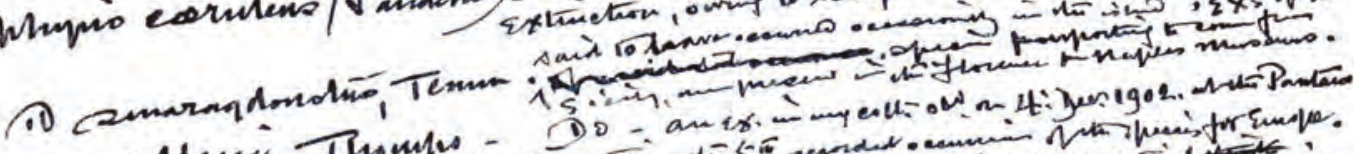

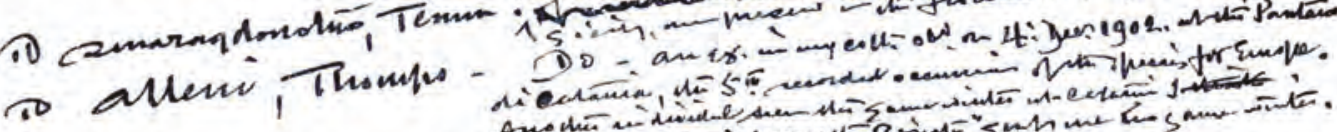

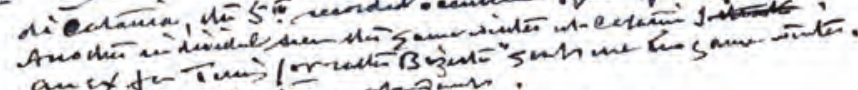

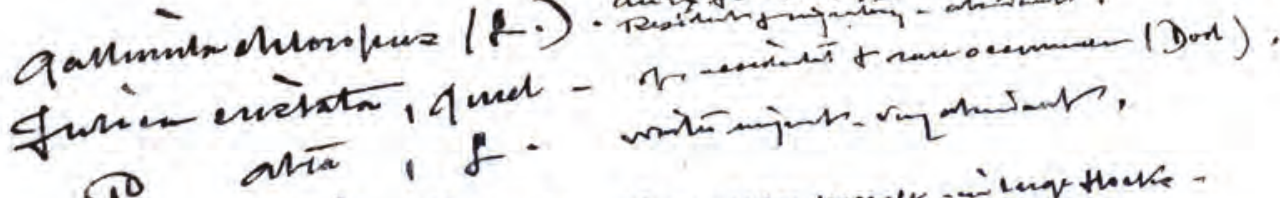

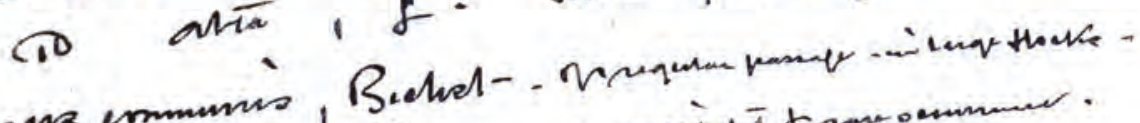

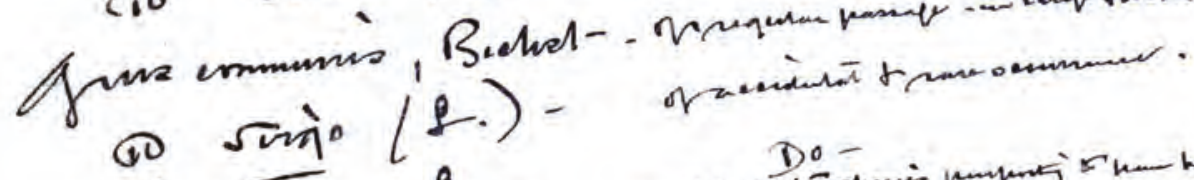

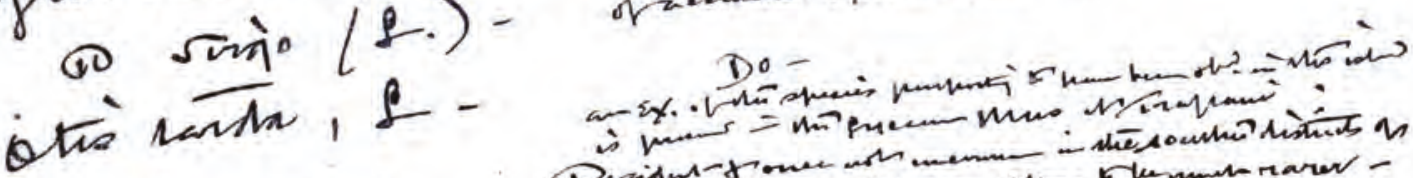

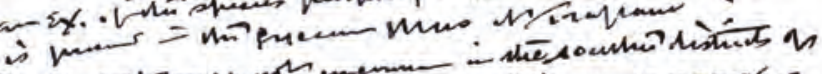

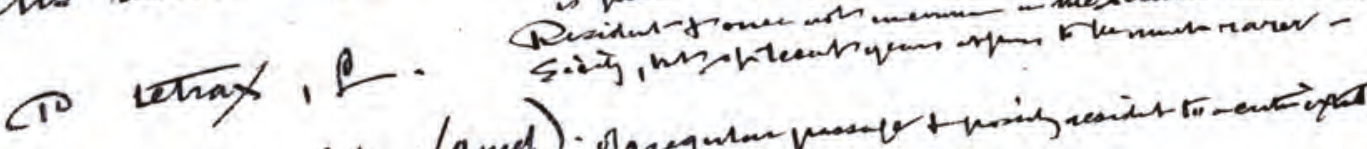

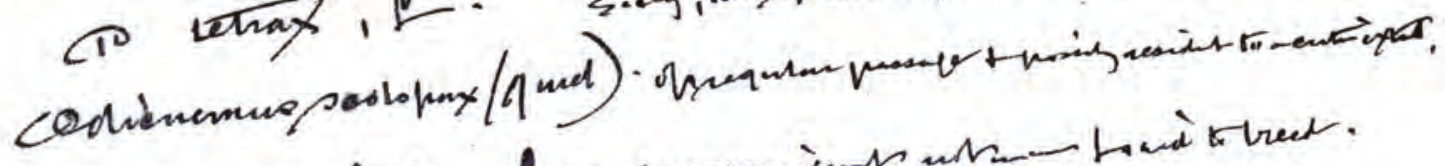

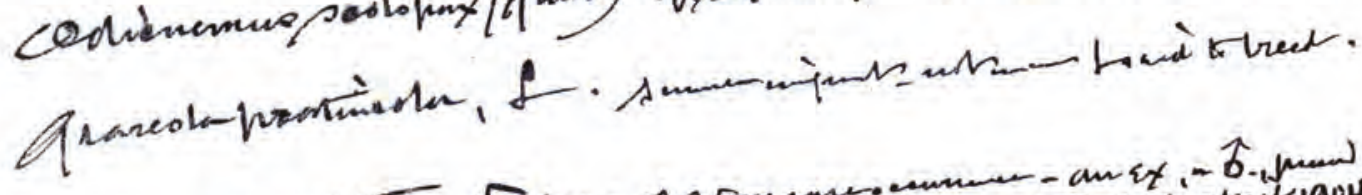

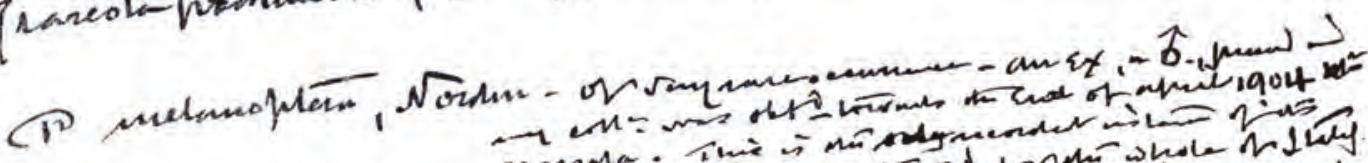

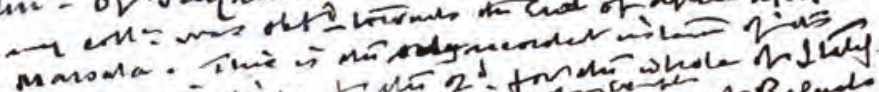

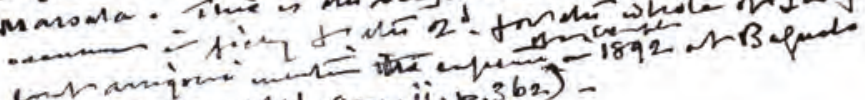
ind a 


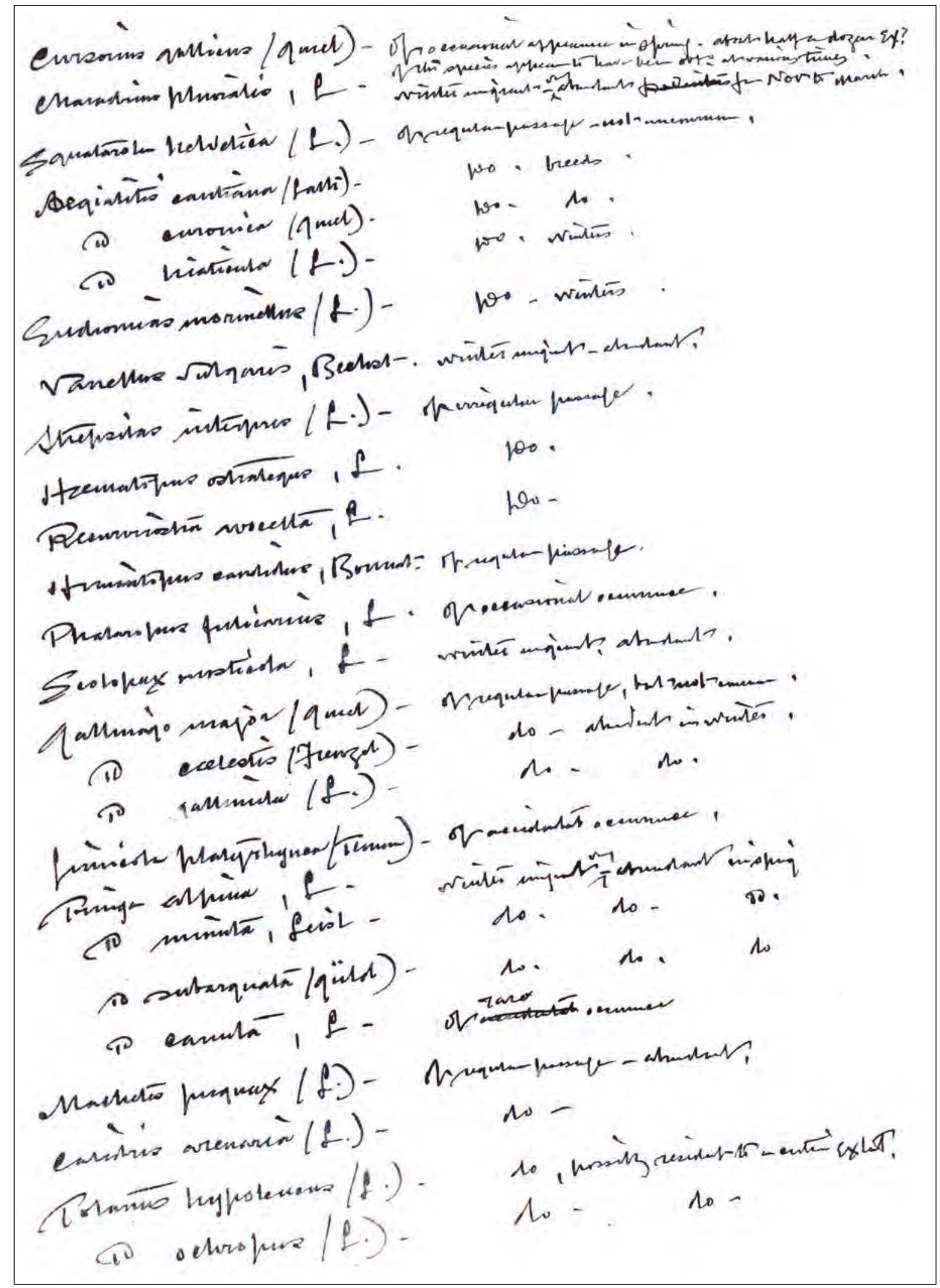




\section{(a).}

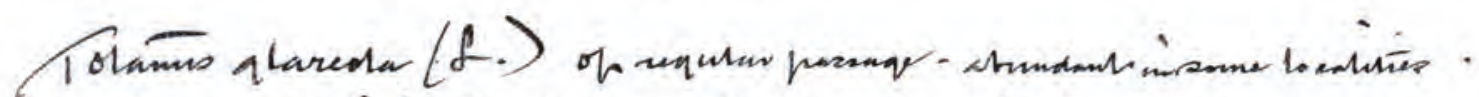

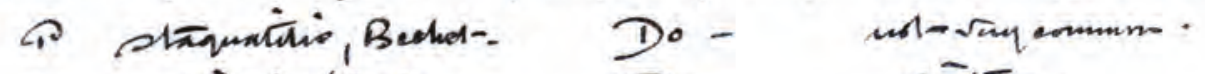

(1) eationis $(\mathcal{f})$. Do winters.

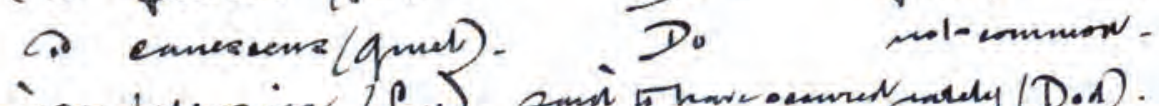

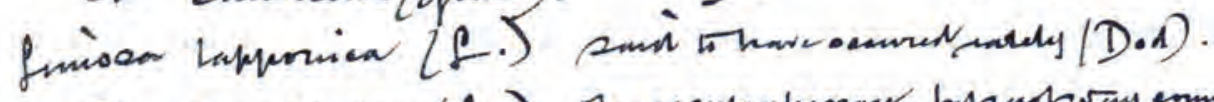

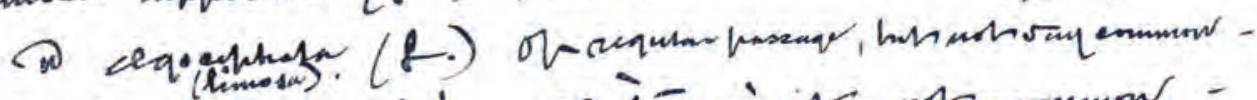

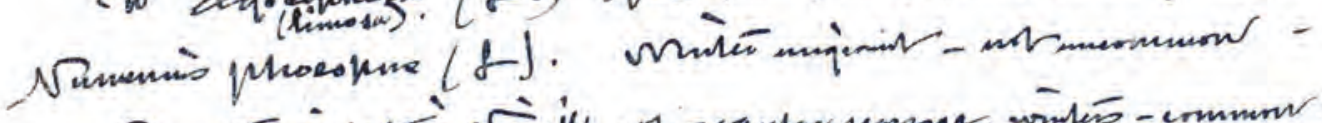

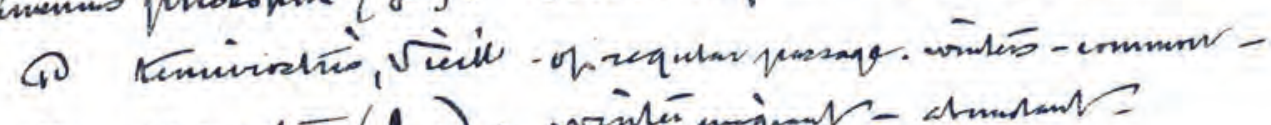

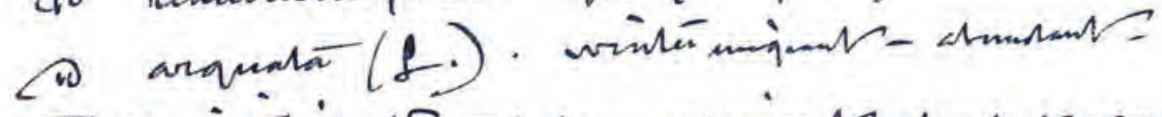

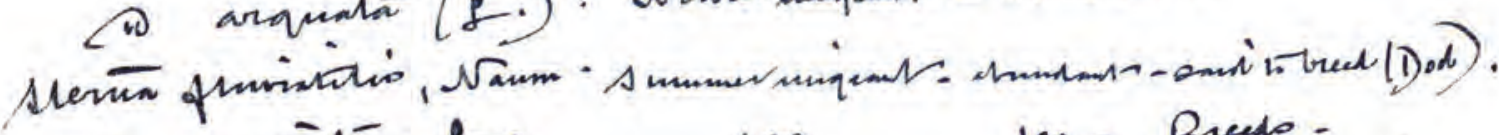

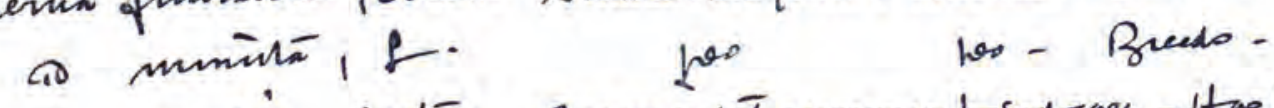

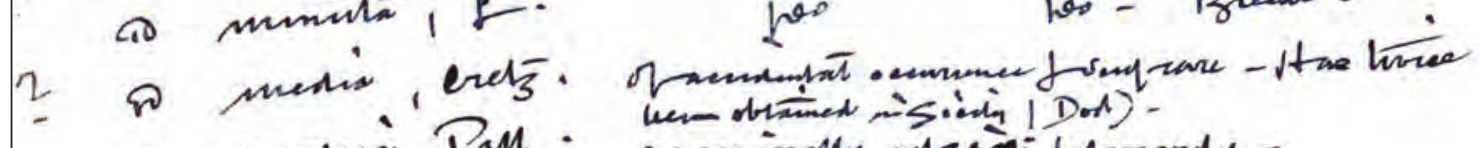

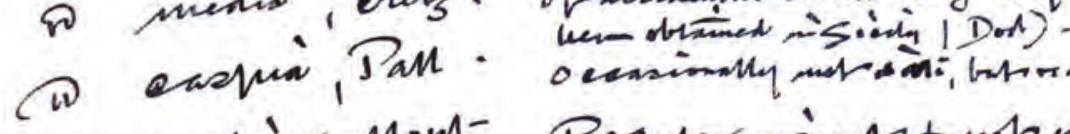

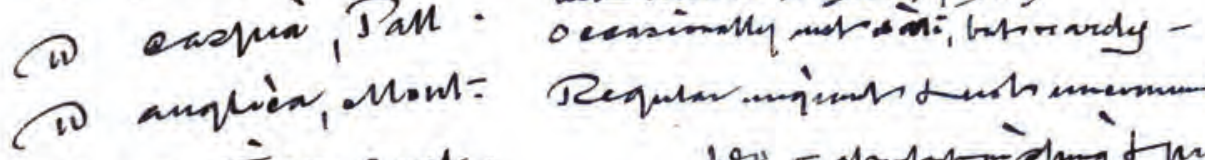

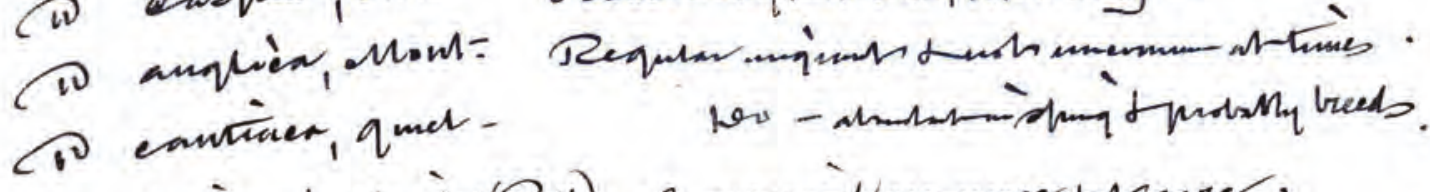

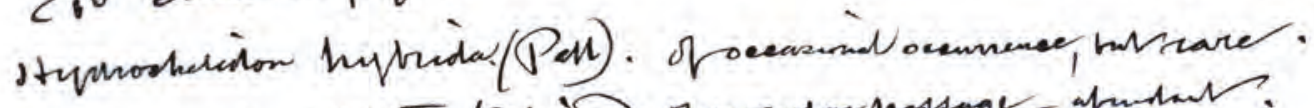

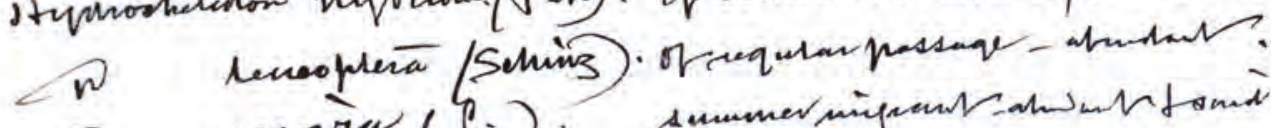

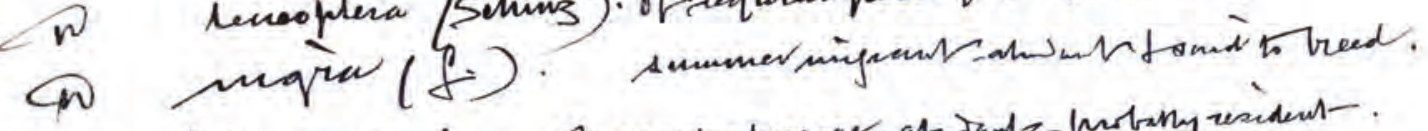

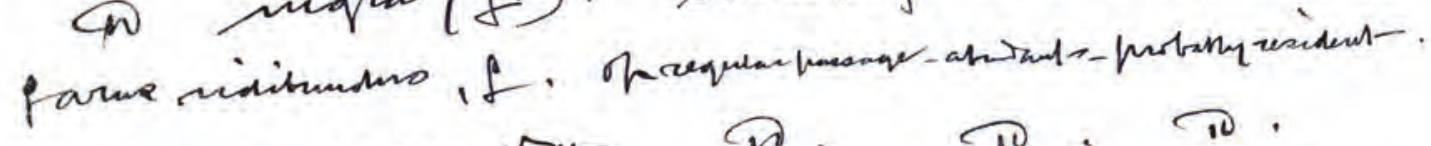

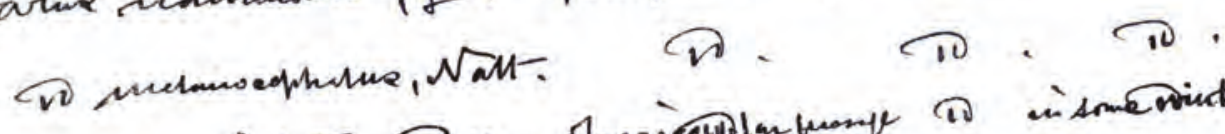

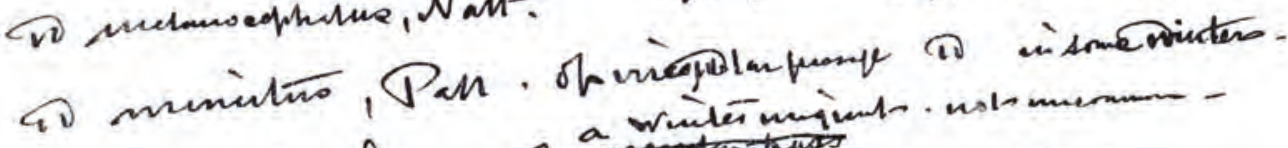

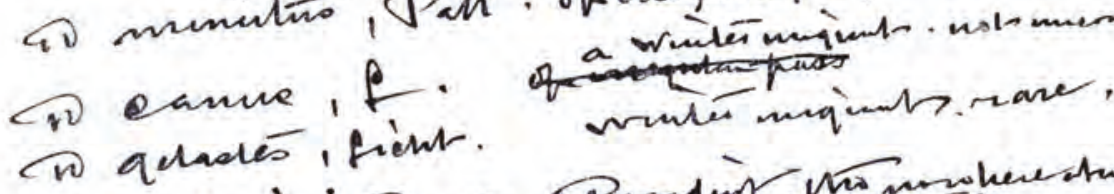

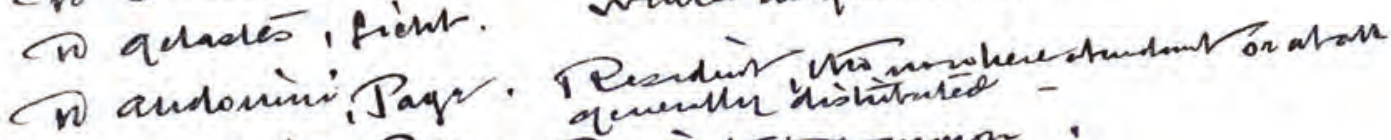

W caechenians, Pall. Geandints armmon.

of troene, fo. Wo.

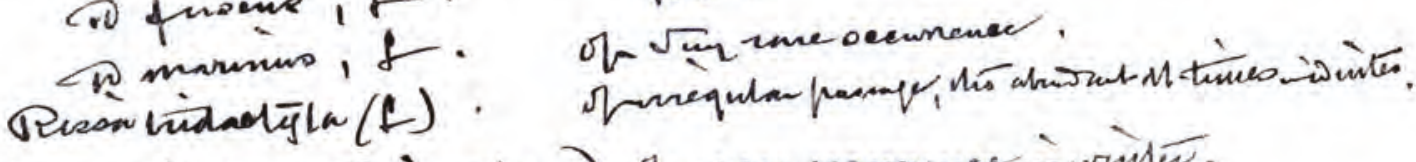

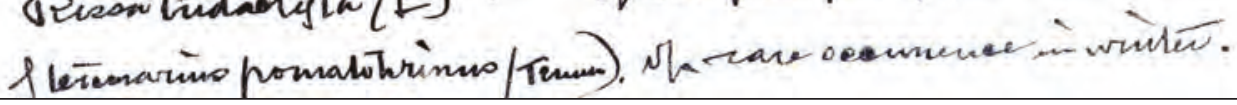




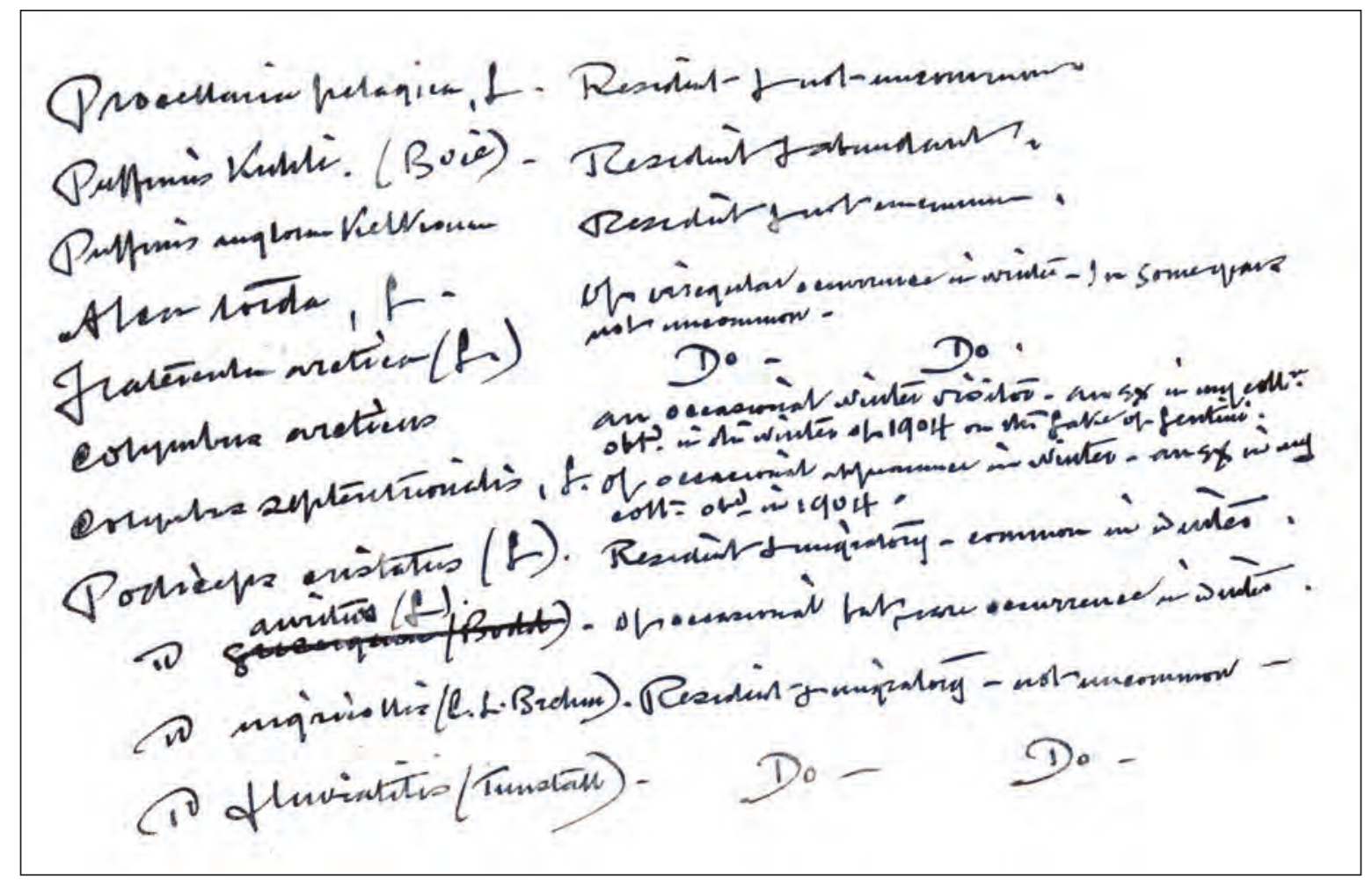

Figure 35. Page 18 of Birds of Sicily by Joseph Whitaker (ca. 1920).

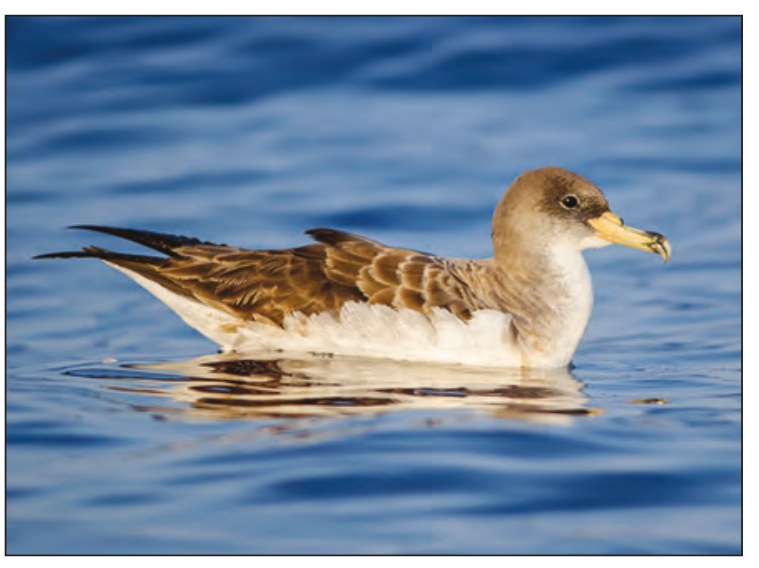

Figure 36. Scopoli's Shearwater Calonetris diomedea. The island of Linosa (Sicilian Channel) houses one of the most abundant colonies of this seabird (photo by T. Puma).

\section{ACKNOWLEDGEMENTS}

We would thank very much Tommaso La Mantia, who since many years has undertaken with the first author long constructive debates on the status of birds in Sicily. We thank also Attilio Carapezza,

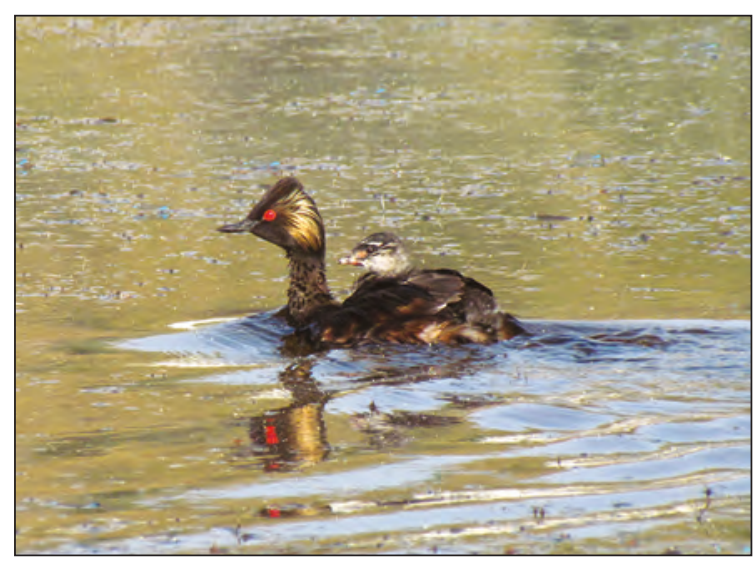

Figure 37. Black-necked Grebe Podiceps nigricollis. Presently in the lake of Pergusa one of the most numerous Italian populations of this grebe breeds (photo by R. Termine).

who helped us during the transcription of the manuscript of Whitaker. We also are indebted with many ornithologists and birdwatchers who in the last years contributed notably to the growth of Sicilian ornithology, in particular Salvatore Baglieri, Antonino Barbera, Vincenzo Billeci, Andrea Cai- 
rone, Giuseppe Campo, Daniela Campobello, Emanuela Canale, Carlo Cappuzzello, Carmela Cardelli, Giulia Casamento, Andrea Ciaccio, Fabio Cilea, Mathia Coco, Andrea Corso, Giovanni Cumbo, Camillo Cusimano, Andrea Cusmano, Davide D'Amico, Stefania D'Angelo, Giacomo Dell'Omo, Nino Di Lucia, Angelo Dimarca, Massimiliano Di Vittorio, Antonino Duchi, Amedeo Falci, Paolo Galasso, Egle Gambino, Giovanni Giardina, Anna Giordano, Emilio Giudice, Salvatore Grenci, Carmelo Iapichino, Ottavio Janni, Giovanni La Grua, Andrea La Mantia, Pietro Lo Cascio, Rocco Lo Duca, Fabio Lo Valvo, Mario Lo Valvo, Lucio Maniscalco, Giuseppe Maraventano, Maurizio Marchese, Angelo Nardo, Nicolò Pitti, Elena Prazzi, Andrea Pulvirenti, Toni Puma, Giuseppe Rannisi, Deborah Ricciardi, Amelia Roccella, Francesco Sanguedolce, Maurizio Sarà, Enzo Sciabica, Angelo Scuderi, Maurizio Siracusa, Gerry Sorrentino, Giovanni Spinella, Rosa Termine and Manuel Zafarana. Finally we thank very much Stefano Maugeri who in 1987 depicted the African Blue Tit in occasion of the fourth Italian Congress of Ornithology held in Pantelleria, Antonino Barbera, Andrea Cairone, Andrea Ciaccio, Mathia Coco, Massimiliano Di Vittorio, Angelo Nardo, Toni Puma, Rosa Termine who authorized the use of their beautiful photographs.

\section{REFERENCES}

Agostini N., Gustin M. \& Panuccio M., 2016. Short-toed snake eagles Circaetus gallicus (Gmelin, 1788) (Aves: Accipitridae) approaching a water barrier show reverse direction of migration. Italian Journal of Zoology, 82: 186-193

Ajola G., 1959. Gli uccelli dell'isola di Ustica. Rivista italiana di Ornitologia, 29: 89-128.

Albores-Barajas Y.V., Massa B., Lo Cascio P. \& Soldatini C., 2012. Night surveys and smell, a mixed method to detect colonies of storm petrel Hydrobates pelagicus. Avocetta, 36: 95-96.

Andreotti A., Aradis A., Berlinguer F. \& Cecolini G., 2020. Il Capovaccaio (Neophron percnopterus) in Italia: fattori limitanti e priorità di conservazione. In Bassi E., Tomasi L. \& Sartirana F. (a cura di), "Info Gipeto" 36. ERSAF Parco Nazionale dello Stelvio ed Ente Aree Protette Alpi Marittime, Bormio 2020 .

Arrigoni degli Oddi E., 1929. Ornitologia italiana. Hoepli, Milano.
Azafzaf H., 2002. Statut actuel de la population de la Cigogne blanche Ciconia ciconia en Tunisie. Alauda, 70: 387-392.

Baccetti N., Fracasso G. \& COI (Italian Ornithological Committee - Rare Birds), 2020. Checklist of the Italian Birds - updated 2019. Avocetta, 44.

Baghino L., Premuda G., Gustin M., Corso A., Mellone U. \& Cardelli C., 2007. Exceptional wintering and spring migration of the booted eagle Hieraaetus pennatus in Italy in 2004 and 2005. Avocetta, 31: 57-62.

Benoit L., 1840. Ornitologia Siciliana. Stamperia G. Fiumara, Messina.

BirdLife International, 2004. Birds in Europe: population estimates, trends and conservation status. Birdlife International, Cambridge, UK.

BirdLife International, 2017. Birds in Europe. Population estimates, trends and conservation status. BirdLife International, Cambridge, UK.

Brichetti P. \& Fracasso G., 2018. The birds of Italy. Volume 1. Anatidae-Alcidae. Ed. Belvedere, Latina (Italy), pp. 512.

Campo G., Collura P., Giudice E., Puleo G., Andreotti A. \& Ientile R., 2001. Osservazioni sulla migrazione primaverile di uccelli acquatici nel golfo di Gela. Avocetta, 25: 185 .

Ciaccio A., 2004. Airone guardabuoi, Bubulcus ibis, e Mignattaio, Plegadis falcinellus, nidificanti in Sicilia. Rivista italiana di Ornitologia, 74: 150-153.

Cortone P., Minganti A., Pellegrini M., Riga F., Sigismondi A. \& Zocchi A., 1994. Population trends of the Red Kite Milvus milvus in Italy. Pp. 29-32 in: Meyburg B.U. \& Chancellor R.D. (eds), Raptor Conservation today. WWGBP \& Pica press.

Cupani F., 1713. Panphyton Siculum. 3 Vol. Ex Typogr. Regia A. Epiro, Palermo (ristampa a cura di Pastena C., Anselmo A. \& Zimmardi M.C., Regione Siciliana).

Di Vittorio, M., Di Trapani, Cacopardi S., Rannisi G., Falci A., Ciaccio A., Sarto A, Merlino S., Zafarana M., Grenci S., Salvo G., Lo Valvo M., Scuderi A., Murabito L., La Grua G., Cortone G., Patti N., Luiselli S. \& López-López P., 2017. Population size and breeding performance of the Lanner Falcon Falco biarmicus in Sicily: conservation implications. Bird Study, 64: 339-343.

Di Vittorio M., Lo Valvo M., Di Trapani E., Sanguinetti A., Ciaccio A., Grenci S., Zafarana M., Giacalone G., Patti N., Cacopardi S., Rannisi G., Scuderi A., Luiselli L., La Grua G., Cortone G., Merlino S., Falci A., Spinella G. \& López-López P., 2019. Long-term changes in the breeding period diet of Bonelli's eagle (Aquila fasciata) in Sicily, Italy. Wildlife Research, 46: 409-414.

Doderlein P., 1869-74. Avifauna del Modenese e della Sicilia. Giornale Scienze naturali ed economiche, 5: 
137-195; 6: 187-236; 7: 9-72; 8: 40-124; 9: 28-93; 10: 35-71, 133-148.

Doderlein P., 1883. Sulla accidentale comparsa di una Sula bassana nelle vicinanze di Palermo. Il Naturalista siciliano, 2: 138-140.

Dresser H.E., 1871-1881. A History of the Birds of Europe including all the species inhabiting the Western Palaearctic Region. Vols. 1-8. Published by the Author, London.

Dresser H.E., 1881. A List of European Birds. Published by the Author, London.

Fasola M., Rubolini D., Merli E., Boncompagni E. \& Bressan U., 2010. Long-term trends of heron and egret populations in Italy, and the effect of the climate, human-induced mortality, and habitat on population dynamics. Population Ecology, 52: 59-72.

Forcina G., Guerrini M., van Grouw H., Gupta B.K., Panayides P., Hadjigerou P., Al-Sheikhly O.F., Awan M.N., Khan A.A., Zeder M.A. \& Barbanera F., 2015. Impacts of biological globalization in the Mediterranean: Unveiling the deep history of human-mediated gamebird dispersal. PNAS, www.pnas.org/cgi/doi/10.1073/pnas.1500677112.

Foschi F., 1968. Monografia sugli uccelli dell'isola di Pantelleria. Rivista italiana di Ornitologia, 38: 1-44.

Giglioli H.E., 1889-1890. Primo resoconto dei risultati dell'Inchiesta Ornitologica. Parte I. Avifauna Italica (1889). Parte II. Avifaune locali (1890). Le Monnier, Firenze.

Giglioli H.E., 1907. Secondo resoconto dei risultati del1'Inchiesta Ornitologica in Italia. Avifauna Italica. Tip. S. Giuseppe, Firenze.

Gill F., Donsker D. \& Rasmussen P. (Eds.), 2020. IOC World Bird List (v10.2). doi: 10.14344/IOC.ML.10.2.

Grenci S., Massa B., Nobile V. \& Rizzo M.C., 1997. Importanza degli Imenotteri (Insecta Hymenoptera) nella dieta del Gruccione Merops apiaster L. (Aves Meropidae). Il Naturalista siciliano, 21: 287-307.

Gretton A., Yurlov A.K. \& Boere G., 2002. Where does the Slender-billed Curlew nest? British Birds, 95: 334-344.

Gustin M., Brambilla M. \& Celada C., 2019. Conoscerli, proteggerli. Guida allo stato di conservazione degli uccelli in Italia. LIPU, 448 pp.

Gutiérrez Expósito C., Copete J.L., Crochet P.A., Qninba A. \& Garrido H., 2011. History, status and distribution of Andalusian Buttonquail in the WP. Dutch Birding, 33(2): 75-93.

Hagemeijer E.J.M. \& Blair M.J., 1997. The EBCC Atlas of European Breeding Birds: Their Distribution and Abundance. T. \& A.D. Poyser, London.

Hutchinson G.E., 1959. Homage to Santa Rosalia or why are there so many kinds of animals? American Naturalist, 93: 145-159.
Iapichino C. \& Massa B., 1989. The Birds of Sicily. British Ornithologists'Union. Check-list n.11, London.

Ientile R. \& Andreotti A., 2003. Primi casi di riproduzione del Pollo sultano, Porphyrio porphyrio, in Sicilia, a seguito del progetto di reintroduzione in corso. Rivista italiana di Ornitologia, 73: 83-86.

Ientile R., Linares A. \& Brogna F., 2016. First breeding colony of Audouin's Gull Larus audouinii in Sicily, characteristics and its origin. Avocetta, 40: 71-76.

Ientile R. \& Massa B., 2008. Uccelli (Aves). In: AA.VV., Atlante della Biodiversità della Sicilia: Vertebrati terrestri. Studi \& Ricerche Arpa Sicilia, Palermo 6: 115211.

Isenmann P., Gaultier T., El Hili A., Azafzaf H., Dlensi H. \& Smart M., 2005. Oiseaux de Tunisie/Birds of Tunisia. Société d'Etudes Ornithologiques de France, Paris, 432 pp.

IUCN (International Union for Nature Conservation), 2017. 2017 IUCN Red List of threatened Species. A global Species Assessment. IUCN, Gland and Cambridge.

La Mantia T., 1994. Regolare nidificazione di Colombaccio, Columba palumbus, in gennaio in Sicilia. Rivista italiana di Ornitologia, 64: 77-78.

La Mantia T., 2001. L'estinzione della Damigella di Numidia Anthropoides virgo (L.) (Aves Gruiformes) a Lampedusa: un esempio di rarefazione della fauna mediterranea. Il Naturalista siciliano, 25: 255-259.

La Mantia T., Buscemi I., Mingozzi T. \& Massa B., 2015. Data analysis of extinct and living Woodpeckers (Aves Picidae) in Sicily and Calabria (Southern Italy). Il Naturalista siciliano, 39: 29-49.

Lord Lilford, 1862. On the extinction in Europe of the Common Francolin (Francolinus vulgaris Steph.). Ibis, Ser. 1 (4): 352-356.

Lo Valvo F., 2001. Aggiornamento delle conoscenze ornitologiche dell'isola di Lampedusa. Il Naturalista siciliano, 25 (suppl.): 121-130.

Lo Valvo F. \& Massa B., 1999. Lista commentata dei vertebrati terrestri della Riserva Naturale Orientata "Isole dello Stagnone" (Sicilia). Il Naturalista siciliano, 23: 419-466.

Lo Valvo F. \& Massa B., 2000. Catalogo della collezione ornitologica Joseph Whitaker (1850-1936). Il Naturalista siciliano, 24 (suppl.): 3-145.

Lo Valvo M., Massa B. \& Sarà M. (red.), 1993. Uccelli e paesaggio in Sicilia alle soglie del terzo millennio. Il Naturalista siciliano, 17 (suppl.): 1-373.

Massa B., 1973. L'Avifauna estiva degli arcipelaghi delle Egadi e dello Stagnone (Trapani, Sicilia). Atti Accademia gioenia di Scienze naturali Catania, 5: 63-95. 
Massa B. (red.), 1985. Atlas Faunae Siciliae. Aves. Il Naturalista siciliano, 9 ( $\mathrm{n}^{\circ} \mathrm{spec}$.): 1-274.

Massa B., 1987a. Joseph Whitaker, ornitologo. Atti Convegno "I Naturalisti e la cultura scientifica siciliana nell'800", 533-540.

Massa B., 1987b. Variations in Mediterranean Crossbills Loxia curvirostra. Bulletin of the british ornithological Club, 107: 118-130.

Massa B., 1995. Storia di un bene culturale espatriato: la collezione ornitologica Whitaker. Atti Convegno "Un Museo di Storia Naturale per la Sicilia", pp. 5760.

Massa B. \& Borg J.J., 2018. European Birds of Conservation Concern: some constructive comments. Avocetta, 42: 75-84.

Massa B., Cerasa G., Bellia E. \& Lo Brutto S., 2018. In ricordo di Pietro Doderlein (2 febbraio 1809-28 marzo 1895). Il Naturalista siciliano, 42: 195-236.

Massa B. \& La Mantia T., 2007. Forestry, pasture, agriculture and fauna correlated to recent changes in Sicily. Forest@, 4: 418-438.

Massa B. \& La Mantia T., 2010. The decline of groundnesting birds in the agrarian landscape of Italy. Revue Ecologie (Terre Vie), 65: 73-90.

Massa B., La Mantia T. \& Rizzo R., 2008. Status ed andamento delle specie d'uccelli nidificanti in Sicilia. In: AA.VV., Atlante della Biodiversità della Sicilia: Vertebrati terrestri. Studi \& Ricerche Arpa Sicilia, Palermo 6: 213-236.

Massa B., Lo Cascio P., Ientile R., Canale E.D. \& La Mantia T., 2015. Gli Uccelli delle isole circumsiciliane. Il Naturalista siciliano, 39: 105-373.

Massa B., Lo Valvo F., Siracusa M. \& Ciaccio A., 1991. Il Lanario (Falco biarmicus feldeggii Schlegel) in Italia: status, biologia e tassonomia. Il Naturalista siciliano, 15: 27-63

Massa B. \& Rizzo M.C., 2002. Nesting and feeding habits of the European Bee-eater (Merops apiaster L.) in a colony next to a beekeeping site. Avocetta, 26: 25-31.

Massa B. \& Sarà M., 2011. Uccelli/Birds. Iconografia della Storia Naturale delle Madonie. Vol. IV. Sellerio ed., Palermo, 375 pp.

Massa B. \& Schenk H., 1983. Similarità tra le avifaune della Sicilia, Sardegna e Corsica. Lavori della Società italiana di Biogeografia, 8: 757-799.

Mebs T., 1957. Ornithologische Beobachtungen in Sizilien. Vogelwelt, 78: 169-176.

Minà Palumbo F., 1857. Aggiunte al catalogo degli Uccelli delle Madonie. La Favilla, Palermo, 1 (10-11): 84-86.

Moltoni E., 1970. Gli uccelli ad oggi riscontrati nelle Isole Linosa, Lampedusa e Lampione (Isole Pelagie, Canale di Sicilia, Mediterraneo). Rivista italiana di Ornitologia, 40: 77-283.
Moltoni E., 1973. Gli uccelli fino ad oggi rinvenuti o notati all'isola di Pantelleria (Provincia di Trapani, Sicilia). Rivista italiana di Ornitologia, 43: 173-437.

Oriani A., 2014. Dati storici sulla presenza circummediterranea del francolino nero Francolinus francolinus francolinus (Linnaeus, 1766). Rivista italiana di Ornitologia, 84: 11-22.

Orlando C., 1935. La Gru pavonina in Italia (Balearica pavonina (L.)). Rivista italiana di Ornitologia, 5: 222-223.

Orlando C., 1958. Il Basettino Panurus biarmicus (L.). Venatoria sicula, 12: 298.

Priolo A., 1967. Distrutti i Grifoni delle Caronie? Rivista italiana di Ornitologia, 37: 7-11.

Priolo A., 1996. Uccelli di Sicilia raffigurati da Cupani nel Panphyton Siculum. Il Naturalista siciliano, 20: 321-410.

Pulvirenti A., 2015. Terza segnalazione accertata di Picchio nero Dryocopus martius martius (Linnaeus, 1758) per la Sicilia. Il Naturalista siciliano, 39: 7172.

Rafinesque C.S., 1814. Arrivo delle Lodole vicino Palermo nell'autunno. Lo Specchio delle Scienze, 132 135.

Raup D.M., 1993. Extinction, bad genes or bad luck? Oxford University Press, Oxford.

Riera E., 1923. Note sugli uccelli osservati nella provincia di Siracusa. Rivista italiana di Ornitologia, 7: 817.

Riggio G., 1892. Corrispondenze scientifiche moderne degli animali figurati, Artropodi e Vertebrati, nel Panphyton Siculum del Cupani. Il Naturalista siciliano, 12: 31-36, 69-73, 115-122.

Robinson J.A. \& Hughes B. (compilers), 2006. International Single Species Action Plan for the Conservation of the Ferruginous Duck Aythya nyroca. CMS Technical Series No. 12 \& AEWA Technical Series No. 7. Bonn, Germany.

Sarà M., Siracusa M. \& Ciaccio A., 1997. Estinzione del Gufo reale, Bubo bubo, in Sicilia. Rivista italiana di Ornitologia, 57: 50-56.

Sciabica V., 2001. Nidificazioni dell'Anatra marmorizzata Marmaronetta angustirostris in Sicilia. Pag. 308 in: Arcamone E. \& Brichetti P., Nuovi avvistamenti. Avocetta, 25: 305-317.

Sciabica E., 2004. Nuovi dati sulla nidificazione dell'Anatra marmorizzata, Marmaronetta angustirostris, in Italia. Rivista italiana di Ornitologia, 74: 166-167.

Schenk H., Chiavetta M., Falcone S., Fasce P., Massa B., Mingozzi T. \& Saracino U., 1985. The Ecology of the Peregrine Falcon in Italy: first results from five sample areas. ICBP Technical Publications, 5: 367-380.

Steinbacher J., 1955. Sull'ecologia e distribuzione degli uccelli in Sicilia. Rivista italiana di Ornitologia, 25: 42-68. 
Stresemann E., 1943. Die Brutvogel des Sees von Lentini, Sizilien. Ornitologische Monabster, 51: 116122.

Surdo S., 2016. Note sui limicoli svernanti nelle zone umide costiere della Provincia di Trapani. Il Naturalista siciliano, 40: 33-49.

Surdo S., 2019. Sulla distribuzione di alcuni uccelli nidificanti in provincia di Trapani (Sicilia). Il Naturalista siciliano, 43: 191-201.

Surdo S. \& Biondi M., 2019. Il Piro piro boschereccio Tringa glareola è uno svernante regolare in Italia. Uccelli d'Italia, 44: 5-9.

Surdo S. \& Matteucci P., 2016. Indagine comparativa sulla popolazione nidificante di Fratino Charadrius alexandrinus (Aves Charadriiformes) nelle saline della Provincia di Trapani. Il Naturalista siciliano, 40: 289-299.

Surdo S., Zafarana M.A. \& Barbera A., 2018. Is the Wood Sandpiper Tringa glareola (Aves Scolopacidae) regular wintering in Sicily? Il Naturalista siciliano, 42: 177-181.

Swainson W., 1836. On the Natural History and Classification of Birds. Vol 2. Lardner's Cabinet Cyclopedia. Longman, Rees, Orme, Brown, Green, and Longman, London.

Triolo S., Campobello D. \& Sarà M., 2011. Diurnal habitat suitability for a Mediterranean steppeland bird, identified by Ecological Niche Factor Analysis. Wildlife Research, 38: 152-162.

Violani C.G. \& Massa B., 1993. Extinction of the Andalusian Hemipode Turnix s. sylvatica (Desf.) in the Mediterranean region. Bulletin of the british ornithological Club, 113 (4): 225-229.

Whitaker J., 1882. Sulla migrazione degli uccelli, specialmente in Sicilia. Il Naturalista siciliano, 1: 121127.

Whitaker J., 1896. Turnix sylvatica in Sicily. Ibis, Ser. 4: 290-291.

Whitaker J., 1899a. Sulla riproduzione in cattività del Pollo sultano (Porphyrio coeruleus Vandelli) volg. sic. gaddo fagiano o gaddu fascianu. Il Naturalista siciliano, 3: 17-20.
Whitaker J., 1899b. Letter on a Caprimulgus aegyptius caught near Palermo. Ibis, Ser. 7: 475-476.

Whitaker J., 1899c. On the breeding of the Purple Gallinule in captivity. Ibis, Ser. 7: 502-505.

Whitaker J., 1899d. The passage of the Cuculus canorus L. in Sicily. Aquila, 6: 99-100.

Whitaker J., 1901. On a new species of Acredula from Sicily. Bulletin of the British Ornithological Club, 11: 51-52.

Whitaker J., 1902. Further information on two recently described species of Passerine Birds. Ibis, Ser.8(2): 54-60.

Whitaker J., 1903. On the occurrence of Porphyrio alleni in Italy and Tunis. Ibis, Ser.8 (3): 431-432.

Whitaker J., 1904a. Il Corvus corone, la Linota rufescens e la Glareola melanoptera in Sicilia. Avicula, 8: 56.

Whitaker J., 1904b. La Glareola melanoptera in Sicilia. Avicula, 8: 84-85.

Whitaker J., 1904c. Notes on Sicilian birds (Letter on Corvus corone, Linota rufescens and Glareola melanoptera in Sicily). Ibis, Ser.8 (4): 477-478.

Whitaker J., 1905. The Birds of Tunisia. Porter, London. Whitaker J., 1910a. On the great invasion of Crossbills in 1909. Ibis, Ser.9(4): 331-351.

Whitaker J., 1910b. Letter on Pterocles senegallus in Sicily. Ibis, Ser.9(4): 567-568.

Whitaker J., 1910c. Cattura di Pterocles senegallus in Sicilia. Avicula, 14: 102.

Whitaker J., 1920. Birds of Sicily. Manuscript dated ca. 1920.

Zafarana M.A., Rannisi G., Grasso R., Spena M.T., Murabito L., Rizzo F. \& Surdo S., 2020. La cicogna bianca Ciconia ciconia in Sicilia: aggiornamento a trent'anni dalla colonizzazione. Il Naturalista siciliano, 44: 95-114.

\section{SITOGRAPHY}

www.ornitologiasiciliana.it/bibliografia 
